\title{
NEAR-THRESHOLD SPUTTERING AND ION TRANSPORT IN TRANSITION METALS
}


So
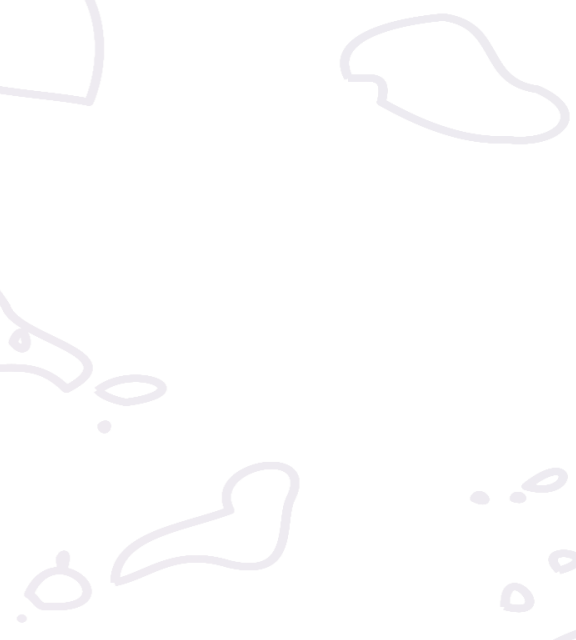

(1)

$-5$.

$\infty$

(1)

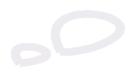

0
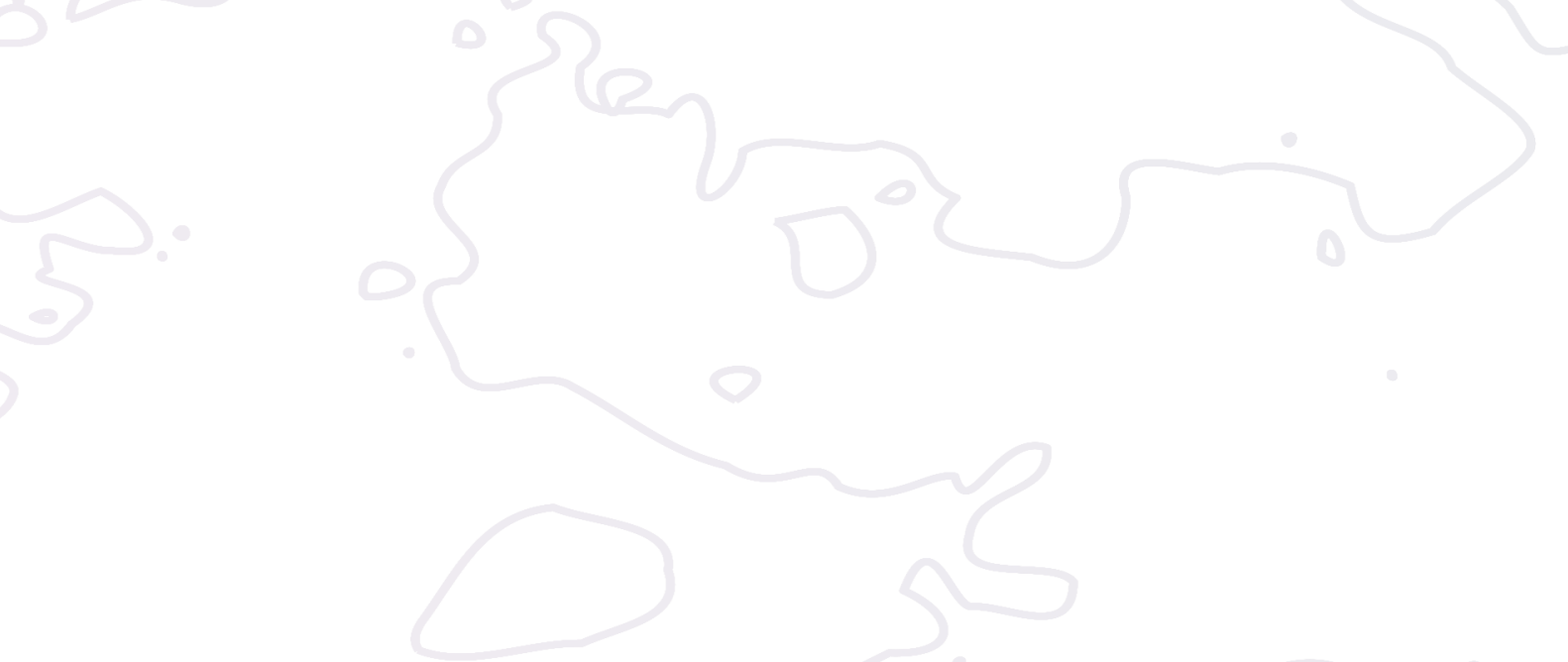

(

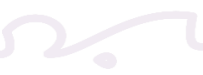

( D

$0<$
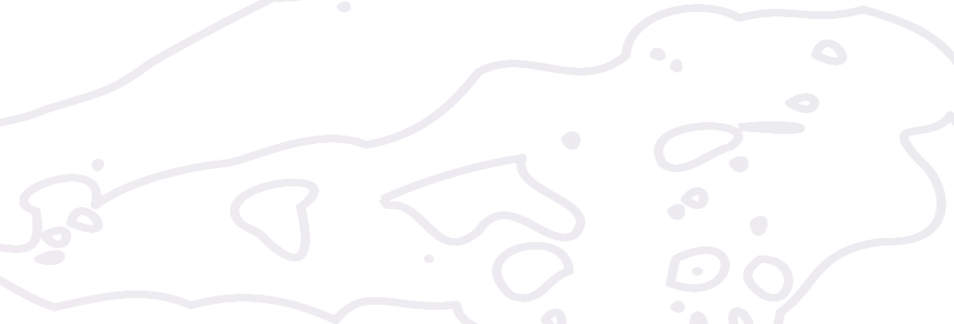

(2)

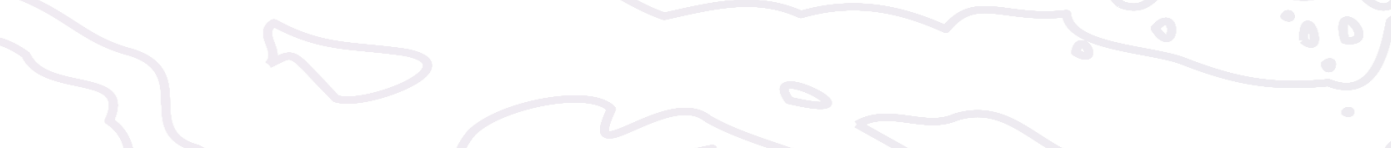




\section{NeAR-Threshold SPUTtering AND \\ ION TRANSPORT IN TRANSITION METALS}

Parikshit Phadke 



\title{
NeAR-Threshold SPUTtering AND \\ ION TRANSPORT IN TRANSITION METALS
}

\section{DISSERTATION}

\author{
to obtain \\ the degree of doctor at the University of Twente, \\ on the authority of the rector magnificus, \\ prof. dr. T. T. M. Palstra, \\ on account of the decision of the Doctorate Board, \\ to be publicly defended \\ on Wednesday $11^{\text {th }}$ November 2020 at 14:45 hours \\ by
}

\section{Parikshit Phadke}

born on the $28^{\text {th }}$ of August 1991

in Bhopal, India 
This dissertation has been approved by:

Supervisor: $\quad$ Prof. dr. F. Bijkerk

Co-supervisor: Dr. ir. J. M. Sturm

\section{Graduation Committee:}

Chairman/secretary:

Prof. dr. J. L. Herek University of Twente, TNW

Supervisor:

Prof. dr. F. Bijkerk University of Twente, TNW

Co-supervisor:

Dr. ir. J. M. Sturm University of Twente, TNW

Committee Members:

Prof. dr. V. Banine Eindhoven University of Technology

Prof. dr. A. von Keudell Ruhr-Universität Bochum, Germany

Prof. dr. J. Schmitz University of Twente, TNW

Dr. H. Wormeester University of Twente, TNW

Prof. dr. M. Ackermann University of Twente, TNW

Keywords: Ion bombardment, sputter yields, nitridation, oxidation, TRIDYN, radiation enhanced diffusion.

Cover: $\quad$ Atomic Force Microscopy contours of metal surface bombarded by ions; design by Jainishka Shah

ISBN 978-90-365-5083-3

DOI: $\quad 10.3990 / 1.9789036550833$

Printed by: Ipskamp Printing 
Copyright (C) 2020 by P. Phadke.

All rights reserved. No parts of this thesis may be reproduced, stored in a retrieval system or transmitted in any form or means without permission of the author.

Alle rechten voorbehouden. Niets uit deze uitgave mag worden vermenigvuldigd, in enige vorm of op enige wijze, zonder voorafgaande schriftelijke toestemming van de auteur.

Acknowledgements:

This work was funded by TNO, the "Nederlandse Organisatie voor Toegepast Natuurwetenschappelijk Onderzoek", and carried out in the Industrial Focus Group XUV Optics at the MESA+ Institute for Nanotechnology at the University of Twente. We acknowledge the additional support by the industrial partners Carl Zeiss SMT, ASML, Malvern Panalytical, as well as the Province of Overijssel and the Netherlands Organisation for Scientific Research (NWO).

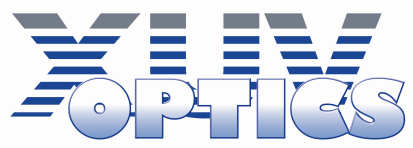 \\ UNIVERSITY | MESA+ OF TWENTE. INSTITUTE}

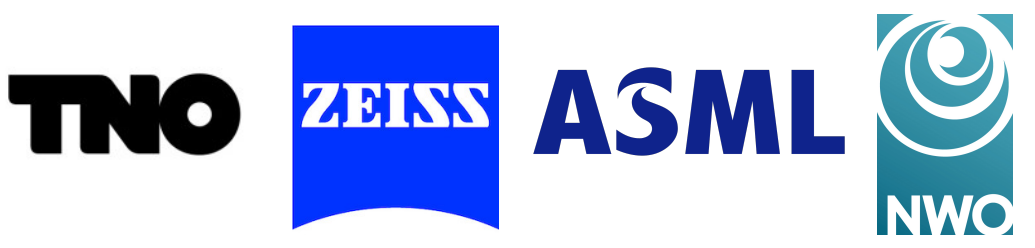

Malvern Panalytical

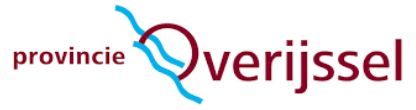



To my parents, And my mentors, in science and in life. 



\section{List of Publications}

This thesis is based on the following publications:

Chapter 3: P. Phadke, J.M. Sturm, R.W.E. van de Kruijs, and F. Bijkerk, "Nearthreshold sputter yields of ruthenium under argon and nitrogen ion bombardment," Nuclear. Inst. and Methods in Physics Research B 458, 169-173 (2019)

Chapter 4: P. Phadke, J.M. Sturm, R.W.E. van de Kruijs, and F. Bijkerk, "Sputtering and nitridation of transition metal surfaces under low energy, steady state nitrogen ion bombardment," Appl. Surf. Sci., 505, 144529 (2020)

Chapter 5: P. Phadke, C.R. Stilhano vilas Boas, J.M. Sturm, R.W.E. van de Kruijs, and F. Bijkerk, "Oxidation and sputtering of transition metals by oxygen ions at steady state: Sputtering and radiation enhanced diffusion near sputter threshold," Appl. Surf. Sci. 146143 (2020)

Chapter 6: P. Phadke, A.A. Zameshin, J.M. Sturm, R.W.E. van de Kruijs, and F. Bijkerk, "Sputter Yields: A Bayesian analysis of semi-empirical models," in preparation. 



\section{CONTENTS}

List of Publications $\quad 9$

1 Introduction $\mathbf{1 5}$

1.1 Motivation ....................... . . 16

1.2 Ion-Surface Interactions . . . . . . . . . . . . . . . . . . . 17

1.2.1 Sputter Yields. . . . . . . . . . . . . . . 17

1.2.2 Ion Transport. . . . . . . . . . . . . . . 18

1.3 Structure of this thesis . . . . . . . . . . . . . . . . . 19

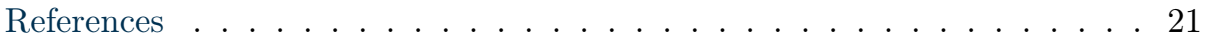

2 Sputter Yield Experiments: Roadblocks and Considerations 25

2.1 Introduction. . . . . . . . . . . . . . . . . . . 26

2.2 Experimental Considerations. . . . . . . . . . . . . . . 27

2.2.1 Base Pressure . . . . . . . . . . . . . . . . . 27

2.2.2 Working Pressure and Ion Flux . . . . . . . . . . . . . 28

2.2.3 Beam Composition . . . . . . . . . . . . . . . 30

2.3 Roadblocks for Broad Beam Ion Sources . . . . . . . . . . . . . . . . 34

2.3.1 Ion source design . . . . . . . . . . . . . . . . . . . 34

2.4 Concluding Remarks . . . . . . . . . . . . . . . . . . . 40

References ....................... . . 42

3 Sputtering of Ruthenium by Argon and Nitrogen 47

3.1 Introduction. . . . . . . . . . . . . . . . . . . . . 48

3.2 Experimental Details . . . . . . . . . . . . . . . . . . 48

3.3 Theoretical Models . . . . . . . . . . . . . . . . . . . 50

3.4 Results and Discussion . . . . . . . . . . . . . . . . . . 51

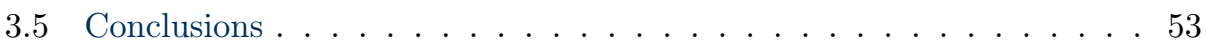

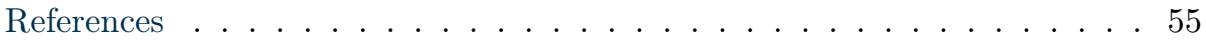

4 Sputtering and Nitridation: XPS Reconstructions 59

4.1 Introduction. . . . . . . . . . . . . . . . . . . 60

4.2 Experimental methods . . . . . . . . . . . . . . . . . . 60

4.2.1 Ion exposure facility . . . . . . . . . . . . . . 61

4.2.2 X-Ray photoelectron spectroscopy. . . . . . . . . . . 62

4.3 Model and simulations . . . . . . . . . . . . . . . . . . 62

4.3.1 Simulating sputtering and implantation depth with TRIDYN . 63

4.3.2 Reconstructing XPS intensities . . . . . . . . . . 65

4.4 Results . . . . . . . . . . . . . . . . . . 66

4.4.1 Sputter yields. . . . . . . . . . . . . . . 666 66

4.4.2 Nitrogen incorporation . . . . . . . . . . . . . . 68 
4.5 Discussion. . . . . . . . . . . . . . . . . . . 72

4.5.1 Sputtering and compound formation . . . . . . . . . . 72

4.5.2 Nitrogen reaction probability . . . . . . . . . . . . . 74

4.6 Conclusions . . . . . . . . . . . . . . . . . . . . . . . . . . . . . . . . . . . . . . . . .

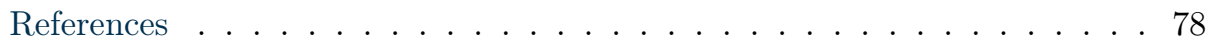

$5 \quad$ Sputtering and Oxidation $\quad \mathbf{8 3}$

5.1 Introduction. . . . . . . . . . . . . . . . . . 84

5.2 Experimental Methods . . . . . . . . . . . . . . . 85

5.3 Model and Simulations . . . . . . . . . . . . . . . . . 86

5.3.1 TRIDYN simulations of sputter yields and depth profiles . . . 86

5.3.2 Multi-Overlayer Model . . . . . . . . . . . . . . . 88

5.4 Results and Discussion . . . . . . . . . . . . . . . . . . . . 88

5.4.1 Sputter Yields from QCM Measurements. . . . . . . . . 88

5.4.2 Chemical modifications: Oxygen ions vs. Atomic Oxygen . . . 93

5.4 .3 Oxide Thickness . . . . . . . . . . . . . . 97

5.5 Conclusions . . . . . . . . . . . . . . . . . . . . . 102

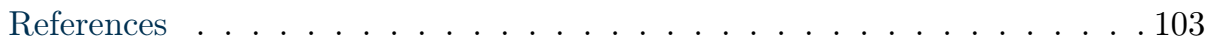

6 Yamamura Model: A Bayesian Analysis 109

6.1 Introduction. . . . . . . . . . . . . . . . . . . . . 110

6.2 Semi-Empirical Formula . . . . . . . . . . . . . . . . . 111

6.2.1 Yamamura Model. . . . . . . . . . . . . . . . 111

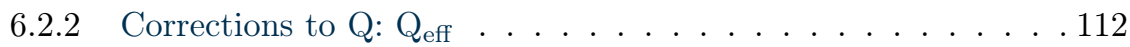

6.3 Bayesian MCMC . . . . . . . . . . . . . . . . . . . . 112

6.4 Experimental Method . . . . . . . . . . . . . . . . . . . . 114

6.5 Results and Discussion . . . . . . . . . . . . . . . . . . . 114

6.5.1 Experimental sputter yields and model parameters: $\mathrm{Ar}^{+}$. . . 114

6.5.2 Experimental sputter yields and model parameters: $\mathrm{Ne}^{+}$. . . 117

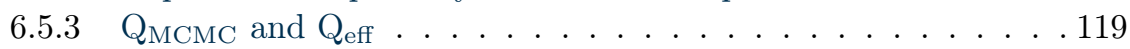

6.6 Conclusions . . . . . . . . . . . . . . . . . . . . . 123

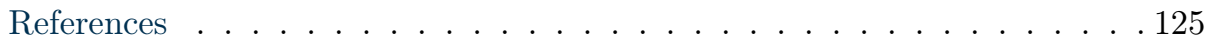

A Appendix Ion Beam Nitridation and Oxidation Effects 129

A.1 Nitrogen ion beam effects . . . . . . . . . . . . . . . . . 130

A.2 Oxygen Ion Beam Effects . . . . . . . . . . . . . . . . . . . 131

B Appendix Bayesian MCMC Results 133

B.1 Posterior Probabilities . . . . . . . . . . . . . . . . . . . 134

B.2 MCMC results for elements . . . . . . . . . . . . . . . . 137

$\begin{array}{ll}\text { Valorization and outlook } & 141\end{array}$

References ......................... . . . . . . . . . . . . . . .

$\begin{array}{ll}\text { Summary } & 143\end{array}$

$\begin{array}{ll}\text { Samenvatting } & 145\end{array}$

$\begin{array}{lr}\text { Acknowledgements } & 149\end{array}$ 
Curriculum Vitae

151 


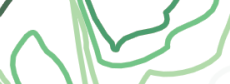

: (2)

: (2)

(2) 2 (1)

(c).

(0) $(0)$

3010010 (0)

$\int 0$

)

$\circ$

10

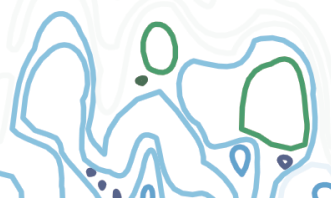

(D)

$0^{0}$

i.

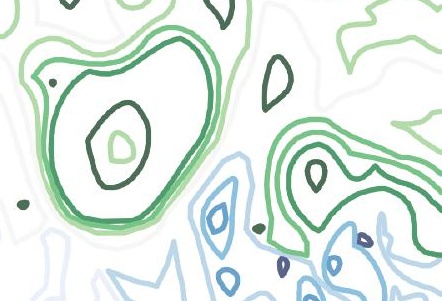

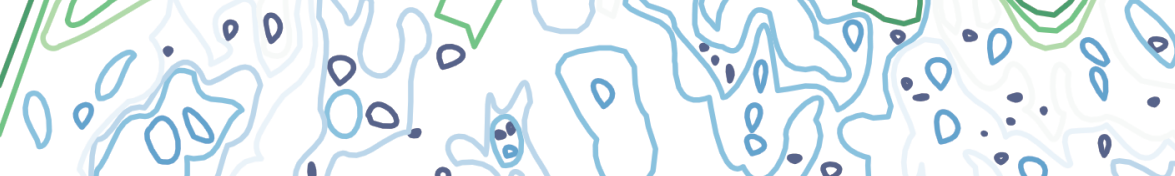

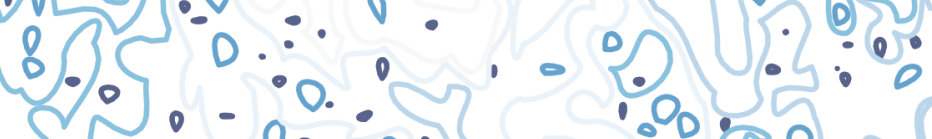

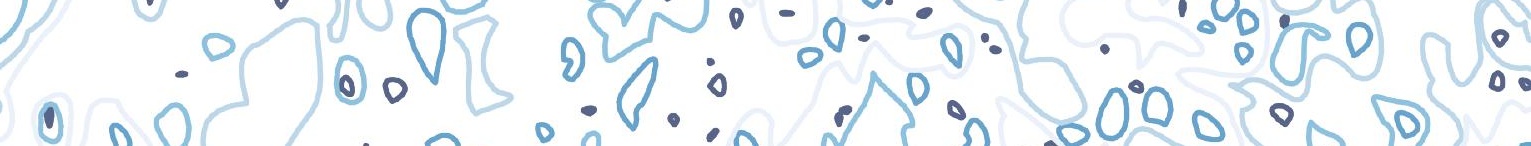

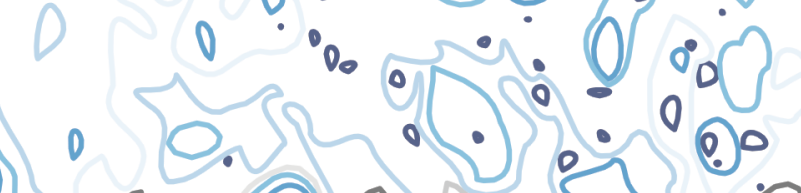

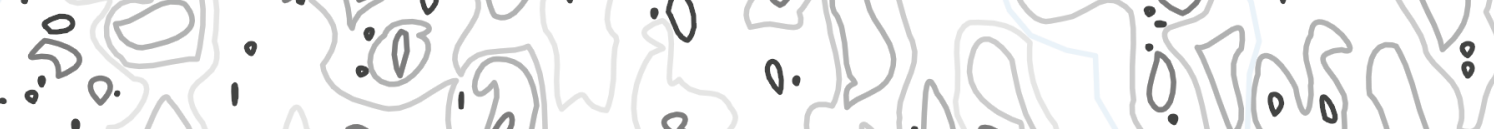

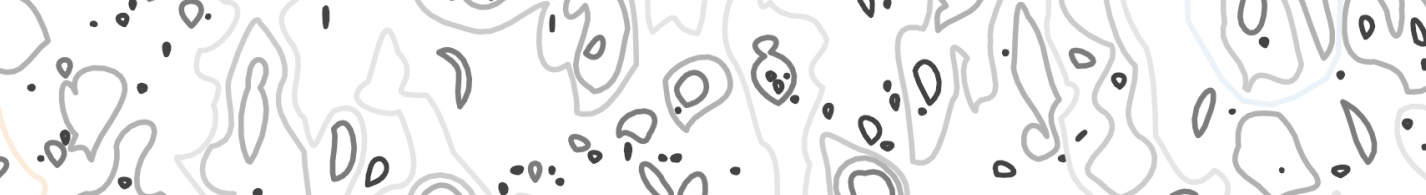

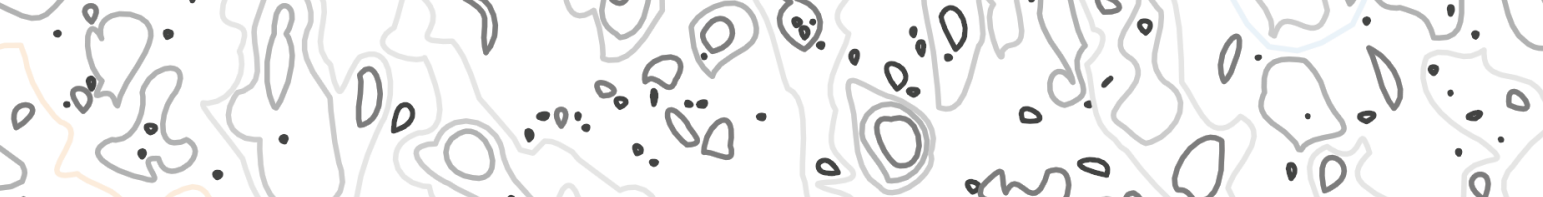

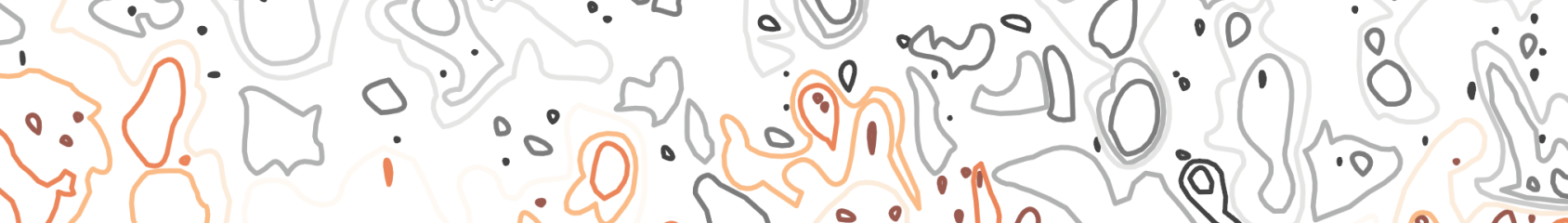

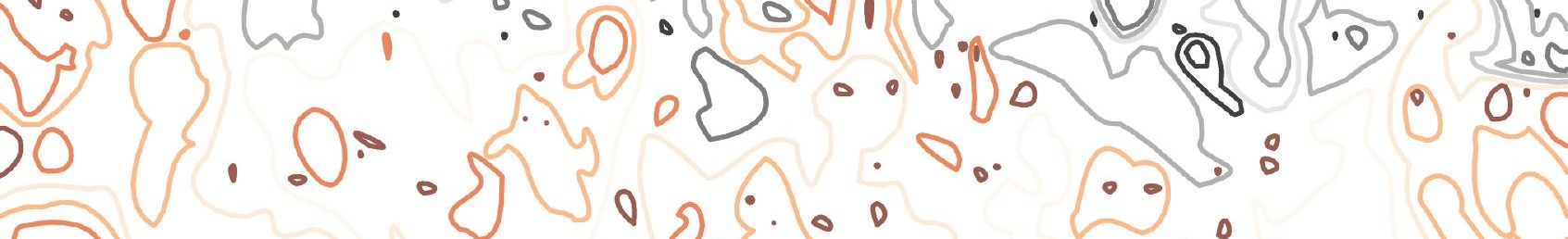

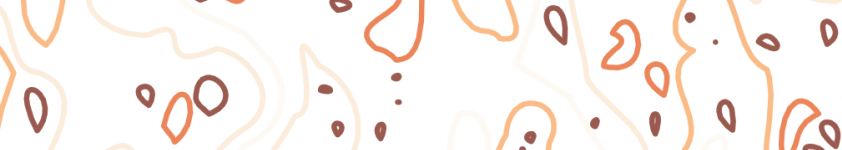

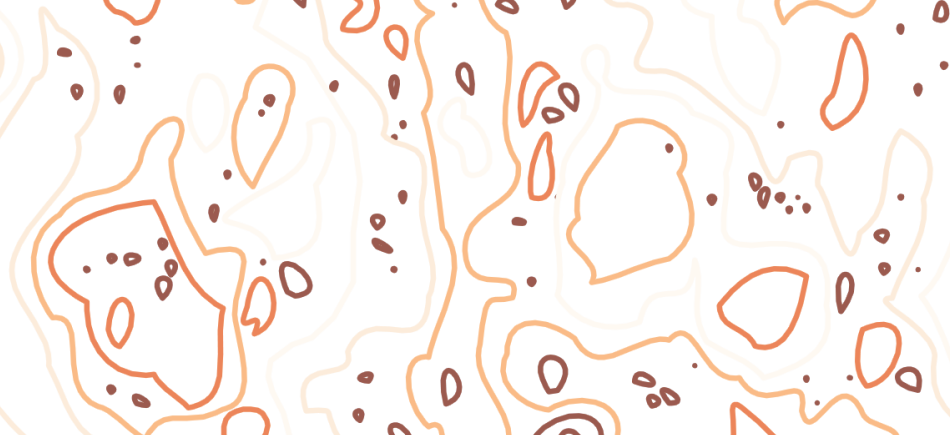




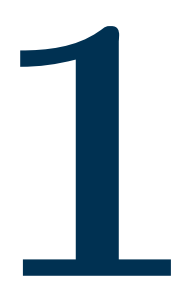

\section{INTRODUCTION}

I may not have gone where I intended to go but I think I have ended up where I needed to be.

Douglas Adams

The long dark tea-time of the soul 


\subsection{Motivation}

The past century brought with it a growing demand for advancement in computational and fabrication tools. It also necessitated an improvement in materials and energy production to sustain these tools. Increasing computational power backed by the semiconductor industry revolutionized nearly every aspect of our daily lives. Semiconductor industries developed techniques to layer complex materials on silicon wafers for high-volume manufacturing. Fabrication tools for scientific and industrial technologies have simultaneously grown to support mass production. Photolithographic techniques have kept pace with the demand and significant improvements have been achieved moving from visible to extreme ultraviolet wavelengths. Optics required for photo-lithographic applications have consequently adapted from transmissive to reflective which required advances in materials research. Transistors thus, grow ever smaller and faster with ever increasing numbers of them planted on microchips. Without them, communication, healthcare, economic and financial transactions, even logistics for daily consumables would be significantly impeded.

Computational power is predicted to only grow in the future, and the energy demanded for its sustenance grows with it. One of the proposed alternatives to conventional energy sources, considered to keep up with this increasing demand, is nuclear fusion. Here, hydrogen nuclei are brought together to produce helium and energy. While nuclear fusion is promising, the technological feasibility of achieving a fusion power station is yet to be demonstrated. Nevertheless, it is a candidate for significant research and potential.

One of the most crucial sciences that paved the way to the realisation of such technologies is plasma physics, particularly, ion-surface interactions. Ions beams from plasma sources allow for anisotropic chemical and physical processing of materials in a controllable manner; Doping semiconductors is possible using ion beams (of gallium for instance) allowing for depth and concentration control of implanted atoms without modifying surrounding structures; Ion (or plasma) bombardment on metals and semiconducting bulk materials enables coating of surfaces with well controlled thin films (in thickness and composition); Reactive ions can be used to locally modify surface structure, and composition through formation of compounds upon surface interaction. Ion-surface interactions can be desired or undesired depending on the application and level of control over the process. For instance: reflective optics for photo-lithography and thin film semiconductor manufacturing can be manufactured using processes such as ion beam deposition [1], ion polishing [2] and ion induced composition modifications [3] which are usually well understood and desired for controlling properties of the optics. They can also be undesired in plasma facing components where the ion species, energies and fluxes are uncontrolled and interactions are unavoidable. This is especially relevant for fusion applications where a plasma is confined in a chamber. Ions of low energies, here defined as $<1 \mathrm{keV}$ are of particular interest. Ions from the plasma interact with the walls of the chambers, divertors and other components they come in contact with, and understanding the interactions of these processes is necessary to limit or counteract the consequences. Consequences such as plasma cooling, chamber wall modification and plasma pollution to name a few. Understanding ion-surface in- 
teractions is also relevant for photo-lithography applications as the reflective optics need to withstand the diffuse plasmas created by the photons used for lithography. Knowledge of these processes can aid in understanding the limits and alternatives for materials used in application.

This thesis is driven by a need to understand the interaction processes of low energy ions (inert and reactive) for semiconductor, lithography and fusion applications. In the following section, we shall outline some primary interaction processes that are explored in this work.

\subsection{Ion-Surface Interactions}

Ion-surface interactions are processes where charged particles of a certain energy (ranging between $1 \mathrm{eV}$ up to $\sim 1 \mathrm{MeV}$ ) are incident on a surface, commonly termed as a target material. The interaction can be of a physical nature if the energy of the incoming ion is high $(>1 \mathrm{keV})$ or of a chemical nature at low ion energies $(\sim 100$ $\mathrm{eV}$ ) for certain species of ions. While this demarcation is not rigid and an overlap of both types of interactions can be observed, it aids in discerning the dominant process. Chemical interactions include reactions forming compounds (volatile or non-volatile). Physical interactions are of a ballistic nature where ions and target atoms can be considered as rigid spheres. Their effects are dependent on the ion energy, flux, and specie (atomic or molecular) and geometry of collision. Depending on ion energy, physical processes can be subdivided into the single knock on regime (for energies $\leq 10 \mathrm{keV}$ ), linear cascade, and thermal spike $(>1 \mathrm{MeV})$. The rigid sphere approximation holds true for the single knock on and linear cascade regimes, where interactions occur between two atoms (binary collisions) at a time and the interactions of electrons of the target are not significant. In the thermal spike regime, a single incoming ion interacts with multiple atoms within the target. Further, the interactions with electrons within the target are significant and binary collision approximations do not apply.

In this thesis, the focus is restricted to the single knock-on regime using inert and reactive ions of energies $<1 \mathrm{keV}$. These energies are relevant to thin film growth applications, plasma facing optics and fusion reactor components. The interactions at these energies govern the micro-structure, compound formation, and ion trapping in the surface and near-surface regions. Low ion energies are particularly interesting as the ions have a lower velocity and thus, larger interaction times. This leads to effects such as increased sputter efficiencies for molecular ions over atomic ions [4, 5], and enhanced chemical activity due to overall inefficient material removal. Near the sputter threshold, the cross-sections of ion-surface atom interactions are also larger and binary collision approximations may break down. Given such behaviour, sputter yields and retention of ion species are not well studied and will be the primary focus of the thesis. We shall briefly expound on these processes further below.

\subsubsection{Sputter Yields}

Sputtering of target materials can be evaluated in the form of efficiency, commonly termed as sputter yield. It is a ratio which quantifies the number of target atoms 
released, upon bombardment by a single ion. Knowledge of sputter yields aids in predicting material loss from ion-target interactions. Many experimental studies measuring sputter yields and involving reactive and inert ion species incident on various targets have been carried out over an energy range of $\geq 0.5 \mathrm{keV}$. Targets vary from pure elemental targets [6] to compounds (from boron nitride [7] to water ice [8]); and ion species are just as diverse: inert gas ions $[6,9,10], \mathrm{N}_{2}$ [11], $\mathrm{O}_{2}$ [12], $\mathrm{CH}_{4}$ [13] and even $\mathrm{Hg}$ [14] to name a few. Experiments have measured sputter yields by varying: angles of incidence [15] and target temperature [16] and also studying composition changes for multi-elemental targets [17]. However, experimental sputter yields, even for elemental targets, have not been extensively studied at ion energies near the sputter threshold. This leads to imprecise estimates of material loss which can be significant in nearly every application mentioned thus far.

Theoretical understanding in the realm of low ion energies has also advanced. Semi-empirical formulae were developed to predict sputter yields for inert ions incident on elemental targets. Eventually, they were extended to include near-threshold ion energies and molecular effects [18]. Improvements in computational power allowed for intensive calculations of ion trajectories within targets via Monte Carlo (MC) simulations [19-21]. MC simulation algorithms, relying on binary collision approximations, proved to be powerful tools to gain insights in statistical processes such as sputtering near the threshold for layered and compound targets. With increasing complexity, they could also account for sputtering under dynamic compositional changes of the target, which proved useful in assessing target poisoning and ion trapping in plasma facing components. In both semi-empirical and $\mathrm{MC}$ approaches, the predictability was found to be reliable for inert ion species incident on elemental or compound targets. However, the determination of a threshold as well as sputter yields at low ion energies for reactive species requires experiments for validations. Again, the availability of comparisons is limited to a handful of elemental target materials.

This thesis is an attempt at filling in gaps in sputter yield data for ion energies $<0.5 \mathrm{keV}$ incident on metallic targets where knowledge is scarce. This is achieved through experimental measurement of sputter yields of metal films grown on quartz crystal micro-balances. The high sensitivity mass loss measurements allowed for reliable measurements of yields down to $\sim 50 \mathrm{eV}$. Sputter yields for some targetion combinations are reported for the first time. Comparison of experiments to semi-empirical models as well as MC simulations was then directly feasible. Target materials and ion species were chosen to appeal to the applications highlighted above.

\subsubsection{Ion Transport}

While sputtering is important in assessing material loss, changes in composition of the target as a consequence of the ion bombardment are equally important. The transport of ions is usually ballistic in nature where elastic collisions of ions with target atoms scatter the ion into sub-surface regions of the target. This leads to phenomena such as implantation of ions and relocations of target atoms (composi- 
tional changes in the target). Experiments in this aspect of interaction are relatively numerous with ion-target combinations of: nitrogen-tungsten [22, 23], oxygentungsten [24], and oxygen-molybdenum [12] to name a few. MC simulations, with capabilities of tracking depth of ion penetration into the target and dynamic compositional changes through implantations [20], aid in understanding ion transport. However, a vast number of reports are limited to compounds formed, with fewer studies on the depth of ion transport. Additionally, to the best of our knowledge, the ion energies are mostly restricted to $>0.5 \mathrm{keV}$.

In cases of reactive ion bombardment, implanted ions can be transported through diffusive channels, which most MC simulations cannot predict. This has been broadly studied for oxygen transport for silicon and silicon-germanium alloy [25, 26] targets, relevant to the semiconductor industry. Reports on diffusive channels for metals have been observed for energies $>1000 \mathrm{keV}$ [27], however, as far as we know, reports on similar studies are unavailable at low energies.

In this thesis, we aim to build a better understanding of reactive ion transport and compositional modifications by nitrogen and oxygen ion species. Comparing experimental observations to MC simulations allows for estimating the impact of ballistic transport into the target. Where direct information of concentration in depth was not possible, simulated depth profiles of implanted ions was used to reconstruct experimental spectroscopic data. This comparison allowed for estimation of reaction cross-sections of target materials with nitrogen ions over an energy range of $50-500 \mathrm{eV}$. Further, it was found that oxygen transport is not purely ballistic in nature, and a diffusional algorithm was developed which couples to simulated concentration profiles to generate effects of ballistic and diffusional mechanisms in tandem.

\subsection{Structure of this thesis}

Given the deficiencies highlighted above: in sputter yields, validity of semi-empirical models and understanding ion transport, this work aims to remedy those deficiencies. It also attempts to encapsulate otherwise disjointed processes of sputtering and ion transport by comparing MC simulations to experimental observations. The structure of this thesis is as follows:

Chapter 2 discusses and highlights experimental design considerations for studying sputtering phenomena in particular and ion-surface interactions in general. These considerations need to be assessed and addressed prior to any experimentation.

In chapter 3, a comparison between the sputtering of ruthenium under argon and nitrogen ion bombardment is carried out. A comparison of experimental results to predictions by semi-empirical models follows. Ruthenium was found to form nitrides upon ion irradiation.

Chapter 4 follows up on chapter 3 by investigating the compound formation in molybdenum, ruthenium, palladium and tungsten under nitrogen ion bombardment. Sputter yields were experimentally measured for each target and compared to MC simulations (which also track ballistic ion transport). The simulations (used to predict sputter yields) were used to predict ion transport and compared to ex- 
perimental X-Ray photoelectron spectroscopic (XPS) data. Nitrogen retention was found to be independent of ion energy and can be described as a competition between implantation and sputtering.

Chapter 5 assesses the ion interaction processes induced by oxygen ions to the selected transition metals compared to nitrogen ions. Here, chemical processes were found to play a relatively larger role, with ruthenium in particular demonstrating anomalous (high) sputtering. Ion transport was also found to be under-predicted by MC simulations when compared to XPS data, highlighting diffusive transport channels. A diffusion algorithm was developed with MC results as inputs to simulate diffusion of oxygen in metals induced and enhanced by ion bombardment.

Finally, Chapter 6 compares the predictions of a semi-empirical (Yamamura) model to simulated and experimentally obtained sputter yield data - from this work and from literature - for inert ion sputtering near the sputter threshold. It extends the comparison from four transition metals to a wide variety across the periodic table. A Bayesian parameter searching algorithm elucidated correlations in the fit parameters of the Yamamura model. The obtained best-fits for experimental and simulated sputter yields were compared to previous reports. It was found that the parameter for linearly scaling sputter yields exhibits bands when scaled to the energy transfer parameter with periodicity according to filling of electrons in orbitals of the target element. 


\section{References}

[1] E. Spiller, S. L. Baker, P. B. Mirkarimi, V. Sperry, E. M. Gullikson, and D. G. Stearns, High-performance mo-si multilayer coatings for extreme-ultraviolet lithography by ion-beam deposition, Appl. Opt. 42, 4049 (2003).

[2] A. Yakshin, E. Louis, P. Görts, E. Maas, and F. Bijkerk, Determination of the layered structure in mo/si multilayers by grazing incidence $x$-ray reflectometry, Physica B: Condensed Matter 283, 143 (2000).

[3] D. S. Kuznetsov, A. E. Yakshin, J. M. Sturm, R. W. E. van de Kruijs, and F. Bijkerk, Structure of high-reflectance la/b-based multilayer mirrors with partial la nitridation, AIP Advances 6, 115117 (2016).

[4] Y. Yao, Z. Hargitai, M. Albert, R. Albridge, a. Barnes, J. Gilligan, B. Pratt Ferguson, G. Lüpke, V. Gordon, N. Tolk, J. Tully, G. Betz, and W. Husinsky, New Molecular Collisional Interaction Effect in Low-Energy Sputtering, Physical Review Letters 81, 550 (1998).

[5] K. Dobes, P. Naderer, N. Lachaud, C. Eisenmenger-Sittner, and F. Aumayr, Sputtering of tungsten by $n+$ and $n 2+$ ions: investigations of molecular effects, Physica Scripta 2011, 014017 (2011).

[6] N. Laegreid and G. K. Wehner, Sputtering yields of metals for ar + and ne+ ions with energies from 50 to 600 ev, Journal of Applied Physics 32, 365 (1961).

[7] B. Rubin, J. L. Topper, and A. P. Yalin, Total and differential sputter yields of boron nitride measured by quartz crystal microbalance, Journal of Physics D: Applied Physics 42, 205205 (2009).

[8] R. Baragiola, R. Vidal, W. Svendsen, J. Schou, M. Shi, D. Bahr, and C. Atteberrry, Sputtering of water ice, Nuclear Instruments and Methods in Physics Research Section B: Beam Interactions with Materials and Atoms 209, 294 (2003), fifth International Symposium on Swift Heavy Ions in Matter.

[9] R. V. Stuart and G. K. Wehner, Sputtering yields at very low bombarding ion energies, Journal of Applied Physics 33, 2345 (1962).

[10] J. Blandino, D. Goodwin, and C. Garner, Low energy sputter yields for diamond, carbon-carbon composite, and molybdenum subject to xenon ion bombardment, Diamond and Related Materials 9, 1992 (2000).

[11] K. Schmid, A. Manhard, C. Linsmeier, A. Wiltner, T. Schwarz-Selinger, W. Jacob, and S. Maendl, Interaction of nitrogen plasmas with tungsten, Nuclear Fusion 50, 025006 (2010).

[12] E. Hechtl and J. Bohdansky, Sputtering behavior of graphite and molybdenum at low bombarding energies, Journal of Nuclear Materials 123, 1431 (1984). 
[13] T. Ast, T. Pradeep, B. Feng, and R. G. Cooks, Low-energy collisions of methane ions at a fluoroalkyl monolayer surface, Journal of Mass Spectrometry 31, 791 (1996).

[14] G. K. Wehner and D. Rosenberg, Mercury ion beam sputtering of metals at energies 4-15 kev, Journal of Applied Physics 32, 887 (1961).

[15] M. Tartz, T. Heyn, C. Bundesmann, C. Zimmermann, and H. Neumann, Sputter yields of mo, ti, w, al, ag under xenon ion incidence, The European Physical Journal D 61, 587 (2011).

[16] V. Philipps, K. Flaskamp, and E. Vietzke, Enhancement of the sputtering yield of pyrolytic graphite at elevated temperatures, Journal of Nuclear Materials 111-112, 781 (1982).

[17] P. Ho, J. Lewis, H. Wildman, and J. Howard, Auger study of preferred sputtering on binary alloy surfaces, Surface Science 57, 393 (1976).

[18] C. Steinbrüchel, On the sputtering yield of molecular ions, Journal of Vacuum Science \& Technology A 3, 1913 (1985).

[19] J. Zeigler, J. Biersack, and U. Littmark, The stopping and range of ions in solids, The Stopping and Range of Ions in Matter 1 (1985).

[20] W. Möller, W. Eckstein, and J. P. Biersack, Tridyn-binary collision simulation of atomic collisions and dynamic composition changes in solids, Computer Physics Communications 51, 355 (1988).

[21] W. Eckstein, R. Dohmen, A. Mutzke, and R. Schneider, SDTrimSP version 5.00, IPP Report 12/08, Max- Planck-Institut für Plasmaphysik (Hrsg.) 12 (2007), http://edoc.mpg.de/display.epl? $\operatorname{mode}=$ doc\&id $=552734$.

[22] G. Meisl, K. Schmid, O. Encke, T. Höschen, L. Gao, and C. Linsmeier, Implantation and erosion of nitrogen in tungsten, New Journal of Physics 16 (2014), 10.1088/1367-2630/16/9/093018.

[23] U. Plank, G. Meisl, U. von Toussaint, T. Höschen, and W. Jacob, Study of the temperature-dependent nitrogen retention in tungsten surfaces using $X$-ray photoelectron spectroscopy, Nuclear Materials and Energy 17, 48 (2018).

[24] N. V. Alov, Surface oxidation of metals by oxygen ion bombardment, Nuclear Instruments and Methods in Physics Research Section B: Beam Interactions with Materials and Atoms 256, 337 (2007), atomic Collisions in Solids.

[25] S. S. Todorov and E. R. Fossum, Growth mechanism of thin oxide films under low energy oxygen ion bombardment, Journal of Vacuum Science \& Technology B: Microelectronics Processing and Phenomena 6, 466 (1988).

[26] O. Vancauwenberghe, N. Herbots, and O. C. Hellman, Role of ion energy in ion beam oxidation of semiconductors: Experimental study and model, Journal of Vacuum Science \& Technology A 10, 713 (1992). 
[27] P. J. Doyle, K. M. Benensky, and S. J. Zinkle, Modeling the impact of radiationenhanced diffusion on implanted ion profiles, Journal of Nuclear Materials 509, 168 (2018). 


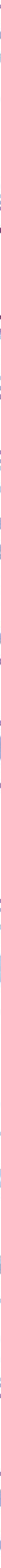




\section{2}

\section{SPUTTER YiELD}

\section{EXPERIMENTS: ROADBLOCKS AND CONSIDERATIONS}

We are stuck with technology when what we want is just stuff that works

Douglas Adams, The Salmon of Doubt

In this chapter we shall discuss the considerations in experimental design and the possible setbacks that influence reliability of sputter yield measurements. Following an overview, we shall extend the list of possible roadblocks with issues faced in this work and provide solutions. 


\subsection{Introduction}

The phenomenon of sputtering was first observed by Grove [1] in 1852 while studying the effects of electric currents in gases. In his work, he studied the effects of oxidation and reduction of the oxide of silver under oxygen and hydrogen discharges, respectively, and reported erosion of the cathode. The work was carried out at a pressure of 16 mbar, considerably higher than those achieved by modern vacuum systems. Grove published his studies along with the note:

"I have given [...] the conditions under which (the experiments) succeeded best; but upon repetition, although the exact volumes of gases and other conditions were carefully attended to, they sometimes required a slight alteration to succeed. [...] it was sometimes necessary to add a little more hydrogen, sometimes a little more oxygen...".

The working pressure, being high, caused irreproducibility. Half a century later, in 1926, Blechschmidt [2] reported his meticulous studies of sputter yields for an extensive collection of metals under $\mathrm{H}_{2}^{+}$and $\mathrm{N}_{2}^{+}$ion bombardment. The experiments were carried out at a pressure of $\sim 0.1$ mbar and thus, were not reproducible. Four decades later, Laegreid and Wehner [3] addressed the issue and reported reliable sputter yields for 28 metals under $\mathrm{Ar}^{+}$and $\mathrm{Ne}^{+}$ion irradiation. Although the high working pressure issue was known in the community before, they were the first to report sputter yields explicitly. Their experiments were carried out in a plasma discharge with a working pressure of $6 \times 10^{-6}$ mbar and limited by contamination at low ion energies where the constant flux of residual gases begins to dominate over the ion flux and leads to mass gain rather than mass loss.

With over a century of efforts directed to studies of sputter yields, the designs of experimental apparatus have evolved to address issues known to exist. Sputter yields in recent times are measured by immersing the target in a plasma [4] or by use of ion sources [5] where the target is placed away from the plasma generator. The loss of material is measured either by capturing sputtered material on a substrate or micro-balance [5] or by measuring mass loss from a target [6, 7].

Experiments carried out for measuring sputter yields impose strict restrictions on vacuum conditions, and knowledge of ion species present in the plasma/ion beam. These have been understood over the years and dedicated experimental systems have been developed involving differential pumping for maintaining ultra-high vacuum, ion beam diagnostics and beam filters. Such dedicated systems offer the advantage of control with limitations on experimental duration as ion fluxes at low energies are low leading to high ion exposure times.

In the work carried out in this thesis, a broad beam source is used for ion generation and sputter yields are measured by monitoring mass loss. Broad beam sources, commonly used for industrial scale etching of surfaces [8] and as ion thrusters for propulsion applications [9], are fitted with grid systems for ion lensing and high flux extraction. Their use for sputter yield experiments is advantageous as they allow for a high ion flux at low ion energies, resulting in shorter experimental duration as well as a homogeneous exposure of the target for ease of (area averaged) characterization and analysis.

We have adhered to experimental measures known to restrict contaminants to 
a minimum which will be discussed in the upcoming section 2.2. However, broad beam ion sources have been, to a lesser extent, studied for applications near the sputter threshold and unforeseen roadblocks may arise from their use at low ion energies. For posterity, we shall outline some of the precautions needed before embarking on projects concerning sputter yields and add to them, insights gained through experimentation with broad beam ion sources.

\subsection{Experimental Considerations}

As discussed in the introductory section above, in order to minimize the expended efforts in measuring sputter yields, some preliminary requirements need to be met. While most parameters discussed below are detrimental to reliability of measured data, some can be easily remedied by thoughtful experimental setups. Others can be remedied through approximations during data analysis while others still, require dedicated metrological tools and equipment. We shall discuss the various parameters in the order of increasing demand for experimental complexity.

\subsubsection{Base Pressure}

Sputter yield measurements need to be performed under high or ultra high vacuum (UHV) conditions. UHV conditions ensure a clean surface is maintained and is subsequently exposed to ion species of choice. The base pressure is a measure of the environmental quality, which the target is subjected to, directly prior to ion exposure and while awaiting analysis (in the case of in-vacuo metrology). Molecules present in the background gas adsorb on the target surface and lead to errors in determining sputter yields. The adsorbed molecules affect the sputter yields more or less severely. At best, the errors are systematic, arising when the ion flux required to remove target material is used up to remove adsorbed contaminants instead. However, at worst, in a combination of more chemically reactive/catalytic target materials with background gas species with chemically active constituents, the ion bombardment may lead to cracking of adsorbed contaminants, resulting in chemical modification of the surface and subsurface regions of the target. Gases like water, or hydrocarbon species present in poorly baked or contaminated systems lead to oxidation [10] of the target or the formation of a carbonaceous layer [11] during ion bombardment, respectively.

The rate of impingement of background gas molecules, $\Gamma$, on the target surface is given by the Hertz-Knudsen equation:

$$
\Gamma=\frac{P}{\sqrt{2 \pi m k_{B} T}}
$$

where $P$ is the partial pressure of the adsorbate, $m$ is its molecular weight, $k_{B}$ is the Boltzmann constant and $T$ is the absolute temperature. For context, a vacuum chamber with water as the dominant background gas, a partial pressure of the order $\sim 1 \times 10^{-9}$ mbar, the target surface is subjected to a background flux of $\sim 4 \times 10^{11}$ molecules $/\left(\mathrm{cm}^{2} \mathrm{~s}\right)$. Consequently, a monolayer of adsorbed water can be expected to form on a target with $1 \times 10^{15}$ sites $/ \mathrm{cm}^{2}$ in a time span of $\sim 3000$ s (50 minutes), 
provided that every incident molecule sticks to the surface. This time required for a monolayer coverage is at least $2 \mathrm{x}$ larger than the duration of most sputter yield experiments. However, assuming a chamber with poor vacuum conditions, say a water background of $\sim 1 \times 10^{-5}$ mbar, a monolayer coverage is achieved in $\sim 0.3 \mathrm{~s}$.

Directly concerning sputter yield measurements, the errors induced by such adsorbing processes depend on the ratio of background gas molecules impinging the surface relative to the ion flux. Anderson and Bay [12] formulated a condition for minimizing the error from adsorption of background impurities. The steady-state concentration of adsorbed impurities $c_{i \infty}$ is given by:

$$
c_{i \infty}=\frac{\Gamma \gamma_{i, s}}{\Gamma \gamma_{i, s}+I Y_{i, s}}
$$

where $\gamma_{i, s}$ is the sticking probability of background gas species $i$ on a substrate $s$. $Y_{i, s}$ is the sputter yield of the adsorbate $i$ when adsorbed on $s$ and $I$ is the ion flux. Demanding a fractional coverage (concentration) of these adsorbates $<10 \%$, we get:

$$
Y_{i, s} I \geq 10 \Gamma \gamma_{i, s}
$$

While equation 2.3 is valid from a kinetic standpoint, the limits are not well studied due to scarcity of data available for sputter yields of adsorbates. However, it is evident from 2.3 that the ability to carry out reliable measurements depends not only on the efficiency of the sputtering, but also on the intensity of the incoming ion beam.

\subsubsection{Working Pressure and Ion Flux}

While the background pressure is necessary for ensuring a clean surface, the considerations for steady state coverage of gas molecules assume a balance between ion flux and background gas flux. In experimental chambers as in this thesis, the sample chamber is not differentially pumped with respect to the plasma discharge chamber. As a result, the flow required for sustaining a discharge leads to a working pressure that is significantly higher than the base pressure ( 4 orders of magnitude). Thus, in addition to the problem of background species being adsorbed, an additional constraint of the working pressure needs to be addressed. The main factors that can be tuned by varying working pressure are as follows:

\section{Mean Free Path and backsputtering}

The mean free path $\lambda$, governs the distance an ejected atom traverses before undergoing a collision with a gas molecule within the experiment chamber and is given (for identical particles) by [13]:

$$
\lambda=\frac{k_{B} T}{\sqrt{2} \pi P d_{m}^{2}}
$$

where, $d_{m}$ is the molecular diameter. Equation 2.4 assumes the interacting particles are near thermal energy. With increasing particle energies, the scattering cross-sections become smaller and $\lambda$ increases. The collision alters the energy and 
direction of the sputtered atom and leads to erroneous yield measurements depending on the experimental design. In experimental designs where ion incidence angle dependent sputter yields are measured by collecting sputtered material on a surface (a quartz micro-balance or a foil), the mean free path presents strict limitations on the reliability of experiments. At grazing angles, ejected target atoms have a preferred direction, which can be altered at high working pressures. Ejected atoms collide with gas molecules and stray away from the collector and are not recorded as sputtered. If the experimental design measures the mass loss instead of collection of sputtered material, a high working pressure (lower mean free path) leads to re-deposition of sputtered material back into the target. In general, having a mean free path smaller or comparable to the source-sample distance leads to deviations in sputter yields and unreliability which is stochastic due to the nature of the collisions involved.

In the work presented in this thesis, mass loss is measured using a quartz microbalance and the working pressure is of the order of $1 \times 10^{-4} \mathrm{mbar}$ at its largest. Assuming the feed gas as argon, the mean free path $(\sim 65 \mathrm{~cm})$ is nearly an order of magnitude higher than the distance between the quartz crystal micro-balance (QCM) and the ion source (Knudsen gas regime). Swapping feed gas to oxygen or nitrogen does not affect $\lambda$ significantly. The large $\lambda$ thus, reduces the possibility of sputtered material to be re-deposited on the target.

\section{Ion Flux}

As we have seen in the previous section, a minimum ion flux is necessary for overcoming the errors caused by background gas adsorption. For low energy experiments, where the ability of the incoming ion to sputter away adsorbates decreases, a high current density (ion flux) is needed to compensate. The ion flux extracted from the plasma depends on the ion energy and design of the ion source. When employing a pin-hole type ion source with a single extraction electrode, the current extracted from the plasma is space-charge limited. This limit is dictated by the Child-Langmuir law [14, 15] which assumes a simple parallel plate configuration for the plasma and extraction grid geometry, with a separation $d$ and an extraction potential $V_{\text {ext }}$ :

$$
j_{\max }=\frac{4}{9} \epsilon_{0} \sqrt{\frac{2 e}{m}} \frac{V_{e x t}^{3 / 2}}{d^{2}}
$$

where $e / m$ is the charge to mass ratio of an ion specie emitted at negligible velocity, and $\epsilon_{0}$ is the permittivity of free space. The parallel plate system is interpreted as the voltage drop between the plasma and the extraction grid. Equation 2.5 sets a restriction to the maximum current density extractable from the plasma for such a geometry. The ion flux scales with the extraction voltage as $V_{e x t}^{3 / 2}$. The extraction voltage in turn determines the ion energy in simple pin-hole ion sources and the current density quickly drops with $V_{\text {ext }}$ and contamination terms in equation 2.3 dominate.

Although equation 2.5 describes a strict limit for maximum ion current density extracted from a pinhole source, this limitation can be circumvented by using broad beam ion sources with efficient extraction systems (grids). The grid system can be 
designed in such a manner as to have multiple, closely spaced apertures (for which equation 2.5 is valid), which work in tandem to produce a higher net extracted current. The limitations placed under such a design arise from geometrical and electrostatic considerations [16].

In order to reduce errors from adsorbate impurities on the surface, we employ a broad beam ion source with a 3 grid lensing system to obtain a high-flux monoenergetic ion beam. The high ion flux ensures a short duration of exposure and therefore shorter experimental time, and minimum adsorbed impurities affecting the results. In addition to the above, the working pressure also dictates the beam composition not only in terms of the species present in the plasma, but also the composition of the particle flux downstream from the ion source. Beam composition can play a significant role in the reliability of sputter yield measurements. We shall now consider the types of ion and neutral species present in gridded broad beam ion sources.

\subsubsection{Beam Composition}

The working pressure and the ion source operation parameters dictate the composition of ions and neutrals in the beam.

\section{Charged Ions and Ion Energy Distribution}

Ionization processes in DC discharges are controlled primarily by the discharge voltage $\left(V_{d}\right) . \quad V_{d}$ is (as a first approximation) the energy imparted to electrons emitted by a filament (cathode). These emitted electrons ionize the feed gas upon impact, creating a plasma. The magnitude of $V_{d}$, when compared to the ionization potential for the feed gas, can help ascertain the presence of doubly charged ion species $\left(\mathrm{Ar}^{++}, \mathrm{Ne}^{++}\right)$. For a more rigorous theoretical assessment, the plasma geometry, electron energy distribution functions and ionization cross-sections from electron/ion impact need to be considered. Experimentally, due to the high neutral pressure (from the feed gas), and low electron temperature $\left(T_{e} \sim 2-4 \mathrm{eV}\right)$, singly charged ion species are dominant. However, by increasing the discharge voltage, some fraction of doubly charged species can be produced. These can affect measured yield values as a doubly charged ion gains twice the energy of a singly charged ion upon extraction, and can therefore sputter more efficiently, especially near the sputter threshold. Once the ions (with a charge $q$ ) from a plasma are extracted by a grid system, the ion energy $\left(E_{\text {ion }}\right)$ is defined by the net potential difference between the extractor grids, and the target $\left(V_{\text {target }}\right)$.

$$
E_{\text {ion }}=q\left(V_{\text {ext }}-V_{\text {target }}\right)
$$

Ideally, the beam energy has a single value (delta function), however, from stochastic processes in the plasma, non-uniformity in the plasma sheath, and collisions with grids, the ion energy broadens. The effect from the presence of doubly charged ions and beam energy spread modifies the measured sputter yield. The modified sputter yield $\left(Y_{\text {mod }}\right)$ can be estimated using [17]: 


$$
Y_{\text {mod }}=\int_{E_{\text {th }}}^{\infty} f\left(E, E_{\text {ion }}, \sigma\right)\left(\left(1-n_{d}\right) Y_{\text {theo }}\left(E_{\text {ion }}\right)+n_{d} Y_{\text {theo }}\left(2 E_{\text {ion }}\right)\right) d E
$$

where $f\left(E, E_{\text {ion }}, \sigma\right)$ is the ion energy distribution, modeled as a Gaussian probability distribution function, with a mean at $E_{\text {ion }}$ and a energy spread $\sigma \cdot n_{d}$ is the fraction of doubly charged ions in the beam and $Y_{\text {theo }}$ is the theoretical sputter yield and $E_{t h}$ is the sputter threshold energy. Equation 2.7 allows for the convolution of the energy spread in the ion beam over the yield, and the effect of doubly charged ions through a weighted average.

Errors in the measured yields obtained from comparing $Y_{\text {theo }}$ and $Y_{\text {mod }}$ are shown in Figure 2.1. For $Y_{\text {theo }}$, the semi-empirical Yamamura model [18] is used, for an ion-target combination of argon-on-ruthenium.

We calculate the deviation in $Y_{\text {mod }}$ with respect to $Y_{\text {theo }}=$ Yamamura as a function of incident ion energy for a doubly charged ion fraction of $1 \%$ and $5 \%$. The spread in the ion energy is set to experimental values with a $\sigma \sim 2-4 \mathrm{eV}$, observed in this thesis work.

It is evident that a significant distortion is observed near the sputter threshold where the errors over small changes in ion energy can vary between a factor 2 and an order of magnitude. However, the yield is most sensitive to variations in doubly charged ion species near the threshold rather than deviations in the energy spread of the incoming ions. In the case of $\mathrm{Ar}^{+}$and $\mathrm{Ne}^{+}$as feed gases used in this study, the discharge voltage used was held at $60 \mathrm{~V}$ and $80 \mathrm{~V}$ respectively. The ratio of electron impact cross-sections for $\mathrm{Ar}^{2+} / \mathrm{Ar}^{+}$and $\mathrm{Ne}^{2+} / \mathrm{Ne}^{+}$formation at these discharge voltages [19] are 0.03 and 0.02 , respectively. Therefore, as a zeroth approximation, in our experiments, the beam is expected to contain at most $2-3 \%$ of doubly charged ions.

\section{Energetic Neutrals}

One of the constituents in the ion beam that is affected by the working pressure is the amount of energetic neutrals present in the ion beam. Ions leaving the ion source can interact with gas molecules in the chamber along the path towards the target. This interaction leads to either changes in ion trajectories through scattering collisions or neutralization through charge transfer collisions. Energetic neutrals formed in charge exchange collisions, due to their inherent lack of charge, do not contribute to the measured ion current but can contribute to sputtering. This leads to an over-estimation of sputter yields and thus, the content of energetic neutrals in yield experiments needs to be eliminated or minimized. In the case of broad beam sources, the primary background gas specie is the feed gas and the charge exchange neutralization cross-sections are considered resonant. As discussed in section 2.2.2, the relatively low working pressure ensures the mean free path is an order of magnitude larger than the sample to source distance. In the case of chargeexchange collisions, a different type of interaction is involved (inelastic processes) where the mean free path needs to be modified according to the corresponding (neutralization) cross-section. For ion-neutral collision processes, the neutralization cross-section can be substantially larger than the simple approximation used for 


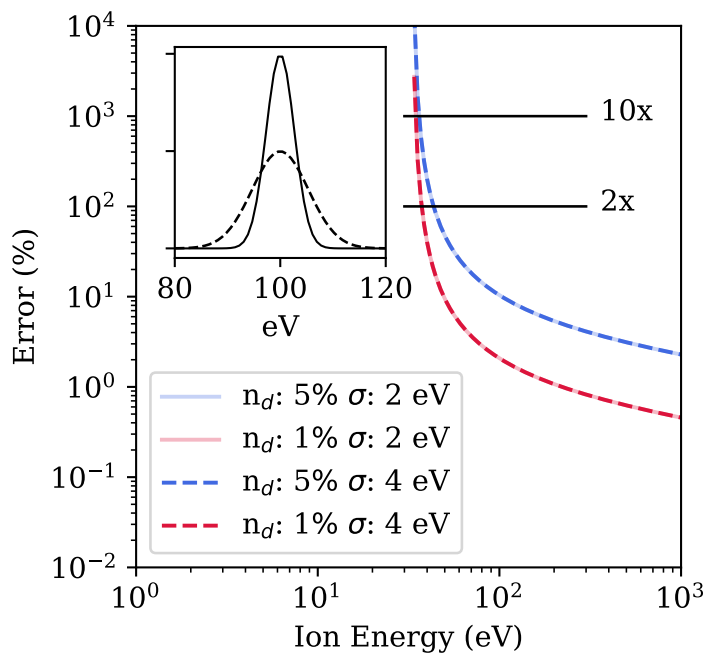

Figure 2.1: Calculated error in measured sputter yields for $\mathrm{Ar}^{+}$on ruthenium considering a certain fraction $\left(n_{d}=1 \%, 5 \%\right)$ of doubly charged ions and an energy spread in the ion beam. The errors in measurements are much less sensitive to the energy spread of the ions over the amount of doubly charged ions that may be present. The black curves (inset) represent the energy spread used in calculations which are set to experimental values from this thesis.

equation 2.4. The current density lost to resonant neutralization processes can be roughly estimated using [20]:

$$
I_{P N}=I_{0} \exp \left(-\frac{P \sigma_{R N} x}{k_{B} T}\right)
$$

$I_{P N}$ is the current density post resonant neutralization, $I_{0}$ is the current density at the source exit plane, $\sigma_{R N}$ is the resonant neutralization cross section. $P$ is the pressure in the chamber (working pressure) and $x$ is the distance between the source exit and the target. Values of $\sigma_{R N}$ were measured for $\mathrm{Ar}$ and $\mathrm{N}_{2}$ at ion energies between $0.5-17 \mathrm{eV}$ [21]. We extrapolate these to the energy ranges used for experiments reported in this thesis, which are shown in Figure 2.2a. The fraction of the current density reaching the detector after resonant neutralization processes is shown in Figure 2.2b.

We find that at our working distance of $7 \mathrm{~cm}$, the current density lost for 50 $\mathrm{eV}$ ions by charge-exchange collisions is $\sim 2 \%$ and $5 \%$ for argon and nitrogen, respectively. Larger working distances, say $1 \mathrm{~m}$, can effectively reduce the measured ion current density by $40 \%$ and cause significant deviations in the experimental sputter yield. The lost current cannot be directly measured by conventional means (e.g. Faraday Cups) as the neutrals (by definition) are not charged. For ion current lost to neutralization, a matching increase in sputter yields can be expected as long as the ion-gas collisions lead to charge transfer only, and do not alter ion trajectories and energy. 
a)

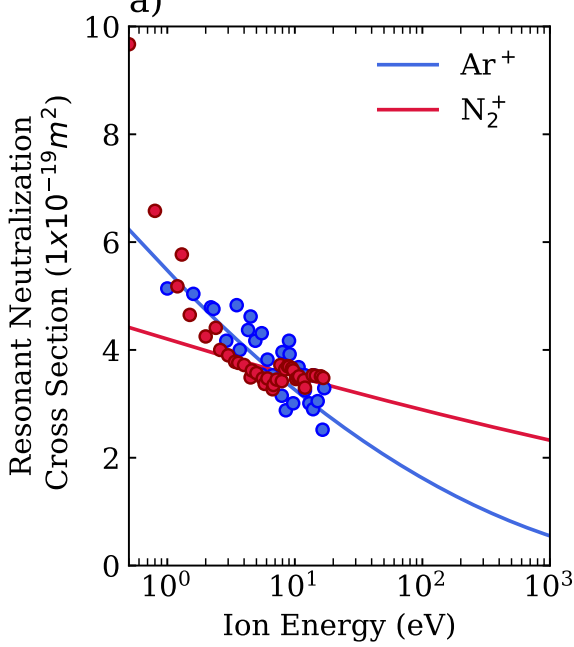

b)

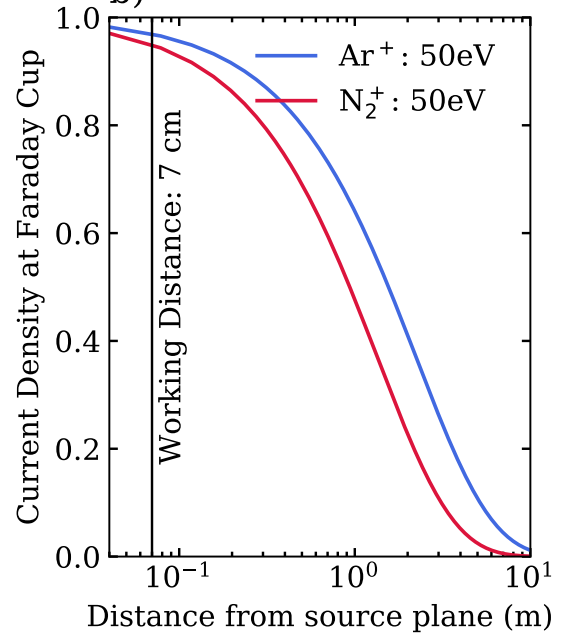

Figure 2.2: a) Resonant neutralization cross-sections for argon (blue circles) and nitrogen (red circles) from [21], extrapolated to working energies in this thesis. b) Calculated ion flux lost to resonant neutralization processes at working pressures $\left(1 \times 10^{-4} \mathrm{mbar}\right)$ for $50 \mathrm{eV}$ ion energy.

\section{Atomic and Molecular Species}

In addition to doubly charged fractions and neutral species, the plasma generated by a molecular feed gas contains atomic as well as molecular species, both of which can be ionized by electron impact. These species can also be found downstream from the ion source. Much effort has been directed towards modeling and measuring the ion species produced in plasmas of hydrogen [22], nitrogen [23] and oxygen $[24,25]$. Models relying on plasma kinetics, while useful, cannot be universally applied without a-priori knowledge of individual reaction pathways for generation and annihilation of every ionic specie in the plasma.

Measurement of atomic and molecular species is a reliable tool for assessing beam contents. The contents of the plasma, which is quasi-neutral, can vary from the contents of the ion beam where (ideally) only the ions are extracted. Techniques for measurements of charged species vary between spectroscopic analysis [26] and quadrupole mass analysis [27]. Additional instruments such as langmuir probes and retarding field energy analyzers aid in determining plasma properties and charged species.

Each method for plasma diagnostics adheres to restrictions, defined by the experimental chamber design, or from detection limits. Spectroscopic techniques, while being fairly simple in experimental set-up, produce results that may be difficult to interpret. Quadrupole mass analysis is another powerful technique which provides quantification of not only atomic and molecular ions in the plasma, but also knowledge on neutrals present in the ion beam. However, for diffuse ion beams/plasmas with low ion kinetic energy energy, measurements become difficult to quantify using off-the-shelf quadrupole analyzers [28]. Further, both techniques require line- 
of-sight of the plasma or ion beam which may not be possible for all experimental set-ups. A combination of retarding field analyzers with quadrupole mass analyzers are optimal for determining the full extent of energetic particles present in the beam. An additional constraint for their operation is the working pressure (as in our experimental setup) which necessitates the use of differential pumping.

For sputter yield experiments, atomic and molecular species can affect the energy transferred to the target atoms and create deviations in measurements. Yao [29] explained in his work that a molecular ion can effectively transfer more energy (than an atomic ion of the same element) when the interaction time of collision between the target and ion is larger than the vibrational half-period of the bond of the molecule. This results in a higher sputter yield for a target when bombarded by a molecular ion over an atomic ion. The onset of a enhanced sputter yield from molecular ion bombardment becomes evident at ion energies $\leq 100 \mathrm{eV}$ and $\leq 250 \mathrm{eV}$ for pure oxygen and nitrogen ion beams, respectively. A knowledge of the fraction of molecular and atomic species in the beam is therefore necessary for experiments near the sputter threshold.

In the present work, the experimental design limits the diagnostic tools used for characterizing ion beam contents. The experimental vacuum chamber did not allow line-of-sight access for spectroscopic or quadrupole mass analysis, therefore we restrict our ion beam characterization to a simple retarding field energy analyzer (RFEA). The RFEA assembly provides knowledge of ion energy and number of charges incident on the target, but no information on atomic or molecular species or neutrals in the ion beam. We rely on the literature on the measurements of atomic and molecular ions generated by similar ion sources to account for our lack of diagnostics. The sputter yields therefore reported, are from a dominant molecular ion beam with atomic ion impurities. The ballistic effects from such a (impure) beam are additionally accounted for in the modeling of yields using a Monte Carlo simulation.

\subsection{Roadblocks for Broad Beam Ion Sources}

In the sections so far, we have enumerated the common factors that plague sputter yield measurements. We have discussed the influence of each parameter on the sputter yield and outlined restrictions in the present thesis as well as remedies for future undertakings. In this section, we shall look into factors, not reported in literature, that were faced in experiments of yield measurements in this work.

\subsubsection{Ion source design}

In the course of developing methodology used in this thesis, we experimented with two types of ion sources with different designs. One was a DC-Kaufman type ion source and the other was a Radio Frequency Inductively coupled plasma (RFICP) ion source. The principle differences in the two discharges were the methods of producing a discharge and the source dimensions. The performance of a DC Kaufman source (Veeco Instruments) with a 3 grid extraction system was compared to a RF-ICP ion source (MicroSystems $\mathrm{GmbH}$ ) with a 3 grid extraction system. 

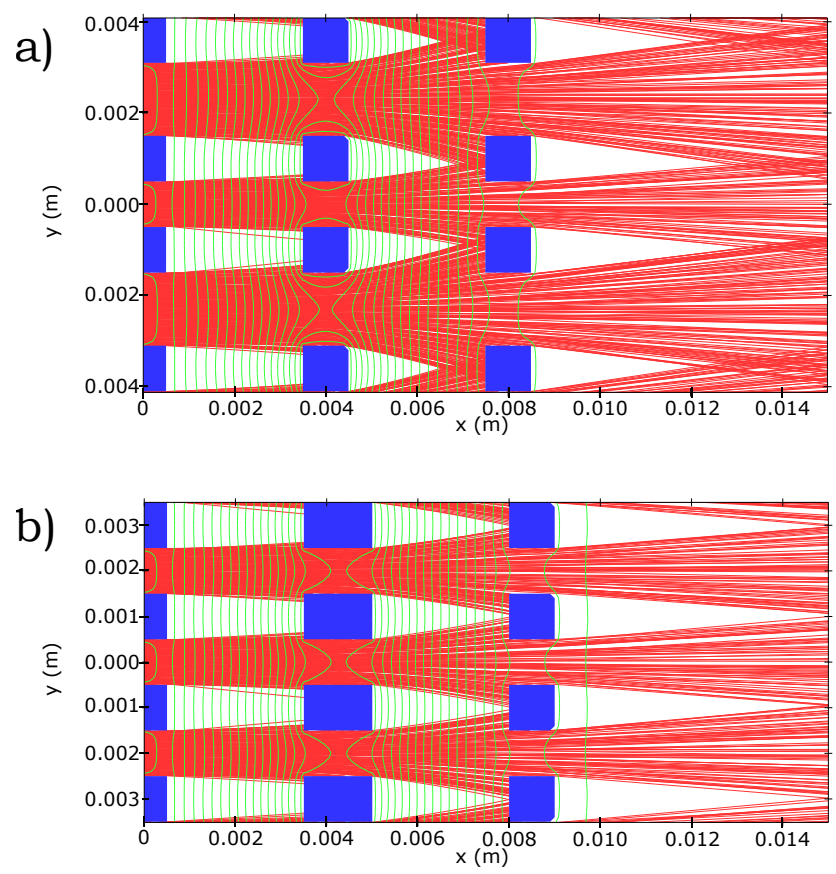

Figure 2.3: Representative configurations of three apertures from a 3 grid geometry for: a) $15 \mathrm{~cm}$ DC ion source; and b) $4 \mathrm{~cm}$ RF-ICP ion source used for test experiments as simulated by IBSimu [30]. The grid assembly consists of a screen grid, an accelerator, and a decelerator. The beam is composed of hundreds of such apertures creating a single broad beam.

The DC source is fitted with a grid system made of molybdenum with a grid aperture diameter of $1.6 \mathrm{~mm}$. The RF-ICP source, having a smaller chamber in which the plasma is contained, is fitted with pyrolytic graphite grids with an aperture size of $1 \mathrm{~mm}$. The spacing between the grids for the DC source was not measured, but is assumed to be similar between the sources and will not play a major role in shaping the ion beam. The aperture sizes do not vary between the three grids of either ion source and ceramic beads used for alignment ensure the apertures in each grid are concentric along the central axis of the source. The geometry and beam behavior, simulated using IBSimu [30], is shown in Figure 2.3.

The grid system is built to extract the plasma and additionally provide lensing. The geometry of the grids can provide a divergent, collimated or convergent grid depending on the radius of curvature of the grid system. The DC source grids were divergent, to ensure a homogeneous beam profile for samples placed at a distance ( $\sim 80 \mathrm{~cm}$ away) while RF-ICP source grids were collimating, where the repulsion of ions downstream is responsible for broadening of the beam profile. Systematic errors in sputter yield due to this grid curvature may occur due to inhomogeneous ion flux over the sample surface, which is typically larger than the diameter of the aperture of the faraday cup as well as the QCM active area. The active area of 
the QCM (which is coated with ruthenium) is $\sim 0.63 \mathrm{~cm}^{2}$ (diameter $0.9 \mathrm{~cm}$ ). The dimensions of the DC source ensured a beam incident on the QCM which was much larger than the QCM active area. On the other hand, the RF-ICP source was measured to produce a beam with a full width at half maximum (FWHM) of $2 \mathrm{~cm}$. Given the QCMs were much smaller than the FWHM of the ion beams, the errors in sputter yield due to grid curvature induced beam profile changes were small.

While the principle of creating a plasma discharge is different between the two sources, the grid system assembly works in a similar manner for both sources. A screening grid provides a boundary for the plasma confinement and shapes the plasma meniscus. The plasma floats against the screen grid. In the case of the DC source, voltage applied to the screen grid translates into an ion energy; while for the RF-ICP source, an electrode (anode) immersed in the plasma within the discharge chamber is biased to impart energy to the plasma and thus, the ions. The accelerator is biased negatively and the decelerator is grounded. The accelerator and decelerator in combination result in efficient ion extraction without imparting additional energy to the ions. From this type of extraction mechanism, no differences (in principle) are expected in the final beam and therefore in the sputter yields.

\section{Differences in Sputter Yields}

Experiments for measuring sputter yields through mass loss were carried out using a QCM. A $400 \mathrm{~nm}$ coating of ruthenium was sputter deposited onto a QCM and placed in front of the ion source $7 \mathrm{~cm}$ away (in both cases). The DC source was run on $4 \mathrm{sccm}$ argon flow (working pressure $1 \times 10^{-4} \mathrm{mbar}$ ) at a discharge voltage of $60 \mathrm{~V}$. The RF-ICP source was run at the same gas flow and pressure conditions, with an input RF power of $150 \mathrm{~W}$. The base pressure in both cases was in the order of $1 \times 10^{-8}$ mbar. The ion energy was varied randomly to avoid systematic errors and the frequency response from the QCM was recorded. In the case of the DC source, a faraday cup simultaneously measured the incident flux on the QCM, while for the RF-ICP configuration, such a simultaneous assessment was not possible. A faraday cup measured the ion flux before and after the QCM exposure and the deviation between the the two measurements $(\leq 5 \%)$ was used to assess the flux during exposure to determine the sputter yield.

The results from experiments using the two sources are shown in Figure 2.4. A simulation using the Monte Carlo code TRIDYN [31] is also plotted for comparison. A stark difference in performance between the two sources is evident from the measurements of sputter yields below $150 \mathrm{eV}$. The RF-ICP source behaves unpredictably at energies approaching the sputter threshold. The yields from the RF-ICP source show an inverted energy dependent behavior with a saturation at $80 \mathrm{eV}$ to a value of 3.8 atoms/ion while the yield obtained from the DC source follow the theoretically expected curve.

The errors enumerated so far cannot directly account for such a drastic change in the sputter yield. While a larger fraction of doubly charged ions can be expected in the RF plasma, the errors induced by their presence can be upto $10 \times$ larger than the predicted value, but not $100 \times$ larger (see figure 2.1). Charge exchange neutrals would induce similar errors in both experiments due to similar working pressures and distances between the ion source and target. The inverse behavior 


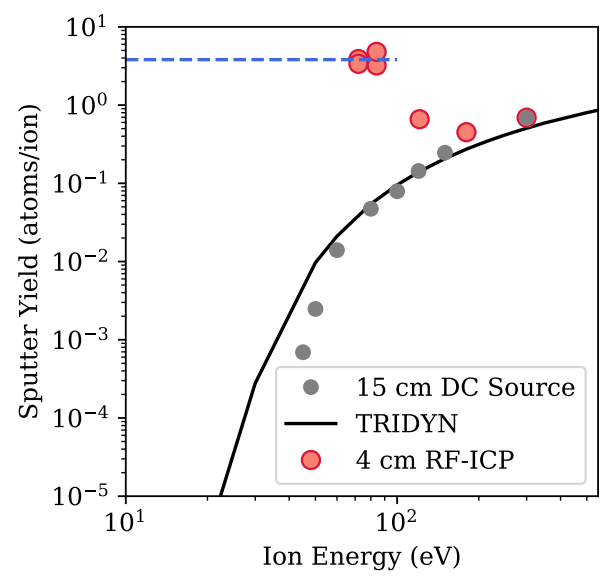

Figure 2.4: Sputter Yields measured using a quartz crystal microbalance coated with a $400 \mathrm{~nm}$ thick ruthenium film for a $15 \mathrm{~cm}$ DC source (gray circles) and a $4 \mathrm{~cm}$ RF-ICP source (red circles). Argon was used as a feed gas in both cases and an expected value from ballistic Monte Carlo simulation using TRIDYN is plotted (solid line). The sputter yields for RF-ICP saturate at a value of 3.8 atoms/ion at energies $\leq 80 \mathrm{eV}$

of the sputter yields as a function of ion energy hints towards processes occurring in the grid system that influence the beam contents significantly. The high sputter yield is indicative of the presence of energetic neutral particles present in the beam that contribute to mass loss. In order to test for energetic neutrals, the sample current density was measured and was found to be $>2 \times$ larger than the current density measured by the faraday cup. Further, experiments with a sample bias were performed by applying a positive potential equal to the incoming ion energy to a witness sample. Nevertheless, sputtering occurred indicating the presence of neutrals in the ion beam.

Looking into the aperture sizes of the grid system, we shall consider neutralization processes before the source exit.

\section{Grid Transparency and Debye Radius}

The hypothesis for the anomalous yields from the RF-ICP source is efficient neutralization, not in the region downstream from the source, but between the individual grid elements. In order to assess the hypothesis, the pressure generated between the grids due to varying conductance of the grid system needs to be determined. We estimate this through the grid transparency which is the effective open area of the grids. This is easily estimated through the ratio of the total area of the individual grid apertures to the area of the grid. The resulting grid transparencies were $32 \%$ and $42 \%$ for the RF-ICP and DC source respectively. The transparency, combined with the area of the grids themselves determine the conductance and therefore, the pressure between the grids. We use the Monte Carlo simulator Molflow + [32] to determine the pressure within the source and chamber for the gas feed used experimentally. The sources were modeled as cylinders consisting of three plates at the vacuum end, representing the grid system, each grid allowing a transparency 
according to the effective open area. A grid spacing of $3 \mathrm{~mm}$ was chosen for both sources.
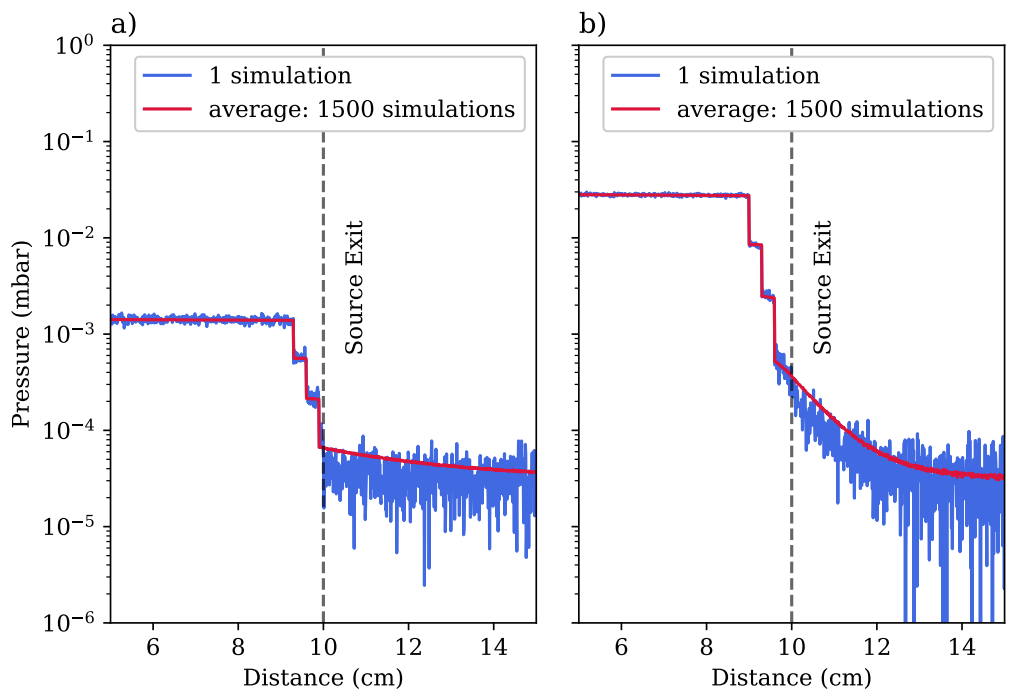

Figure 2.5: Plot showing simulated pressure profile using Molflow + for a) $15 \mathrm{~cm}$ DC source and b) $4 \mathrm{~cm}$ RF source with grid transparencies of $42 \%$ and $32 \%$ respectively.

The pressure profiles form the Molflow + simulations are shown in Figure 2.5. The pressures between the grids for the DC source are about $16 \times$ lower than the pressure built up in the RF-ICP grid assembly. To compare the effect of the pressure buildup, we need to consider the energies of the ions in this interim locations. The energy of ions between the grids is determined by the accelerator potential, which we shall consider to be the nominal value of $300 \mathrm{eV}$. Thus, from a purely chargeexchange collision standpoint, according to equation $2.8,10 \%$ and $85 \%$ of the ions between the screen and accelerator grids are neutralized in the DC and RF-ICP source respectively. Re-ionization of energetic neutrals in the RF-ICP can also occur due to multiple collision events and the estimate of $85 \%$ is an upper bound assuming single collision occurrences. The energy of the ions within this region lies between $\sim q\left(V_{\text {beam }}\right)$ and $q\left(V_{\text {acc }}\right)$, where $V_{\text {beam }}$ is the potential applied to the screen (DC) grid or immersed anode (RF-ICP), and $V_{a c c}$ is the magnitude of the accelerator potential. Neutrals formed in this region will preserve this energy but will not be affected by the decelerator grid. $V_{\text {beam }}$ is roughly the ion energy when using argon as the working gas.

With lowering $V_{\text {beam }}$, the neutral energy increases due to the subsequently larger difference between $V_{\text {beam }}$ and $V_{a c c}$. The design for imparting energy to the ions can also have an effect on the neutral content as the grid system creates a diffuse plasma to fill the chamber and the space between the grids. We shall consider the aspects of the plasma confinement that complicate the presented simplistic assessment, along with solutions in the following section.

In addition to the pressure built up between the grids, broad beam ion sources 
are not efficient in plasma confinement and the generated plasma diffuses into the experimental chamber. The energies of these ions are in the order of the discharge voltage $\left(60-80 \mathrm{eV}\right.$ ) and thus, can neutralize more efficiently (higher $\sigma_{R N}$ ) at these higher pressures and sputter target material. Due to this poor plasma confinement, the simplistic treatment of equation 2.8 is not sufficient at the ion energies where the recombination processes within the plasma can introduce additional pathways for neutralization.

In order to aid in plasma confinement, the apertures of the screening grid need to be modified. The choice of the aperture dimensions is dictated by the debye length, $\lambda_{D}$ in the plasma, defined as [33]:

$$
\lambda_{D}=\sqrt{\frac{\epsilon_{0} k_{B} T_{e}}{n_{e} e^{2}}}
$$

where $n_{e}$ is the electron density in the plasma and $T_{e}$ is the electron temperature. Assuming a dense plasma discharge as can be achieved by an RF source, with $n_{e} \sim 1 \times 10^{17} / \mathrm{m}^{3}$, and an electron temperature of $4 \mathrm{eV}$, the corresponding $\lambda_{D}$ is $47 \mu \mathrm{m}$. In order to obtain optimal electron confinement, the plasma grid aperture dimensions need to be comparable to $\lambda_{D}$ [34]. The RF-ICP source thus allows for the plasma to seep out of the screen grid and can interfere with grid operation and sputter yield measurements. While a similar electron density and a comparable debye length can be expected for DC Kaufman type ion sources with magnetic confinement (for longer electron lifetimes), the larger apertures ensure a more diffuse plasma due to recombination or escape channels for ions and electrons away from the target [34]. Thus, in order to combat the interplay between plasma confinement and pressure between grids, the following solution was implemented.

\section{Working solution: Modified Screen Grid}

We address the issue by modifying the screen grid geometry as shown in the Figure 2.6. A woven mesh of stainless steel with a pore dimension of $40 \mu \mathrm{m}$ is used as the plasma facing grid. In order to limit warping of the mesh from thermal loads of the plasma, the mesh was spot welded on a stainless steel replica of the pyrolytic graphite screen grid (Figure 2.6d). A single $1 \mathrm{~mm}$ aperture of the screen grid contains multiple mesh openings. The beamlet dimension is therefore limited by the aperture of the screen grid. The screen grid is grounded (Figure 2.6g) and the accelerator acts as an extractor for ions and this potential applied, translates to the beam energy. The decelerator is kept at ground potential and a neutralizer filament downstream is used to negate the beam divergence due to space charge.

Sputter yields measured by the modified grid for two probed energies are shown in Figure 2.6f. At energies $<150 \mathrm{eV}$, the initial deviation in the measured sputter yields showed an inverse proportionality to incident energy which saturated at 80 $\mathrm{eV}$. After applying modifications the sputter yields reduced significantly and were in agreement to the predictions by TRIDYN. The data points from the modified grid systems are shown with errorbars to illustrate the errors occurring from sources such as ion flux and energy distribution. These are not as significant in the unmodified case. 

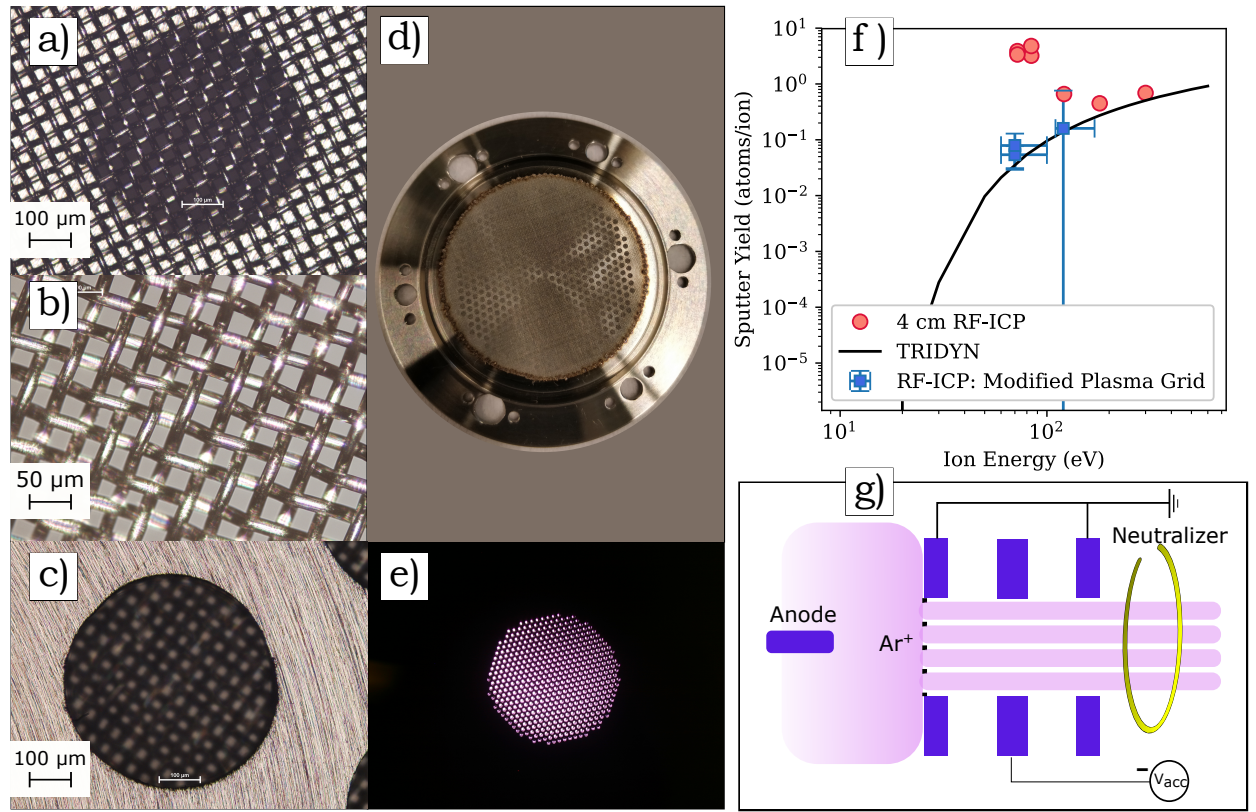

Figure 2.6: Modifications performed on the screen grid of the ion source with a) Plasma facing view of mesh attached to a stainless steel screen grid; b) woven mesh configuration as seen through an optical microscope; c) Target facing view of plasma grid with mesh holes seen on a single aperture; d) modified screen grid with a mesh spot welded on the plasma side and e) argon plasma seen through a viewport after assembling the 3 grid system with modified screen grid. f) Sputter Yields measured before and after modification to grid assembly; g) Schematic of modified grid assembly where the small black squares represent the cross-sections of the mesh wires (Component sizes not to scale).

\section{Limits of Grid modifications}

While the sputter yields obtained from the modified grid assembly are promising, the reliability of the solution was limited. The materials used (stainless steel) induced significant thermal loads onto the ceramic chamber where the discharge was confined. This led to unpredictable swings in ion flux in the time scales of minutes where measurements are carried out. Further, the replica of the screen grid used to prevent warping was misaligned with the remainder of the grids and produced a high energy tail in the ion energy distributions. This leads to errors along the energy axis of the sputter yields (as can be seen in Figure 2.6f). Lastly, the configuration did not allow for a high ion flux at energies below $70 \mathrm{eV}$ as reducing the aperture size (via the mesh) limits the extractable current from the plasma [16]. The modifications however, are a proof-of-concept of modifications possible to decrease unpredictable sputtering behavior of broad beam ion sources.

\subsection{Concluding Remarks}

In conclusion, in order to obtain reliable measurements of sputter yields, experiments and the experimental apparatus need to be designed to minimize sources of 
contamination. For this, a low base pressure $\left(\leq 1 \times 10^{-8}\right.$ mbar $)$ and a low working pressure $\left(\leq 1 \times 10^{-4} \mathrm{mbar}\right)$ are necessary. When using single aperture sources, a differentially pumped vacuum system is optimal for such studies. The contents of the ion beam need to be measured, and if not feasible, realistic estimates of major ion species present need to be made. To study isolated effects of atomic and molecular species in the beam, mass filters can additionally be installed which relaxes the demand for measuring beam contents. Neutral filters, installed downstream from the ion source can be efficient in relaxing the restrictions arising from high working pressure, which might be needed in certain cases to maintain a stable discharge. Finally, in the case of broad beam ion sources with grid systems for ion extraction and lensing, the source behavior can become unpredictable at low ion energies due to grid designs. These can be remedied through plasma confinement to a certain extent. Care must be taken when operating broad beam sources at energies near the sputter threshold as a-priori knowledge of such behavior is not available.

Given the anomalies, working solutions and limitations of the RF-ICP source, we rely on the DC ion source for further experiments carried out and discussed in this thesis. 


\section{References}

[1] W. R. Grove, On the electro-chemical polarity of gases, Philosophical Transactions of the Royal Society of London 142, 87 (1852).

[2] E. Blechschmidt, Kathodenzerstäubungsprobleme. (ii. mitteilung) die kathodenzerstäubung in abhängigkeit von den betriebsbedingungen, Annalen der Physik 386, 999 (1926), https://onlinelibrary.wiley.com/doi/pdf/10.1002/andp.19263862509 .

[3] N. Laegreid and G. K. Wehner, Sputtering yields of metals for ar + and ne+ ions with energies from 50 to 600 ev, Journal of Applied Physics 32, 365 (1961).

[4] D. V. Lopaev, T. V. Rakhimova, A. T. Rakhimov, A. I. Zotovich, S. M. Zyryanov, and M. R. Baklanov, Silicon dioxide and low- $k$ material sputtering in dual frequency inductive discharge by argon ions with energies from 16 to 200 eV, Journal of Physics D: Applied Physics 51, $02 \mathrm{LT} 02$ (2017).

[5] B. Rubin, J. L. Topper, C. C. Farnell, and A. P. Yalin, Quartz crystal microbalance-based system for high-sensitivity differential sputter yield measurements, Review of Scientific Instruments 80, 103506 (2009), https://doi.org/10.1063/1.3249560 .

[6] S. M. Wu, R. W. E. van de Kruijs, E. Zoethout, and F. Bijkerk, Sputtering yields of Ru, Mo, and Si under low energy Ar+ bombardment, Journal of Applied Physics 106, 054902 (2009).

[7] M. F. Doemling, B. Lin, N. R. Rueger, G. S. Oehrlein, R. A. Haring, and Y. H. Lee, Using a quartz crystal microbalance for low energy ion beam etching studies, Journal of Vacuum Science \& Technology A 18, 232 (2000), https://doi.org/10.1116/1.582139 .

[8] H. R. Kaufman, Broad beam ion sources: Present status and future directions, Journal of Vacuum Science \& Technology A 4, 764 (1986), https://doi.org/10.1116/1.573810 .

[9] P. J. Wilbur, V. K. Rawlin, and J. R. Beattie, Ion thruster development trends and status in the united states, Journal of Propulsion and Power 14, 708 (1998), https://doi.org/10.2514/2.5333 .

[10] W. Reuter and K. Wittmaack, An aes-sims study of silicon oxidation induced by ion or electron bombardment, Applications of Surface Science 5, 221 (1980).

[11] F. Naehring, A. Schmidt, and J. Schöneich, Ion induced carbon contamination and recoil implantation, Phys. Status Solidi A 44, K141 (1977).

[12] H. H. Andersen and H. L. Bay, Sputtering yield measurements, in Sputtering by Particle Bombardment I: Physical Sputtering of Single-Element Solids, edited by R. Behrisch (Springer Berlin Heidelberg, Berlin, Heidelberg, 1981) pp. 145218. 
[13] W. Umrath, Fundamentals of vacuum technology, (1998).

[14] C. D. Child, Discharge from hot cao, Phys. Rev. (Series I) 32, 492 (1911).

[15] I. Langmuir, The effect of space charge and residual gases on thermionic currents in high vacuum, Phys. Rev. 2, 450 (1913).

[16] H. R. Kaufman, J. J. Cuomo, and J. M. E. Harper, Technology and applications of broad beam ion sources used in sputtering. part i. ion source technology, Journal of Vacuum Science and Technology 21, 725 (1982), https://doi.org/10.1116/1.571819 .

[17] M. Nakles, Experimental and modeling studies of low energy ion sputtering for ion thrusters, Master's thesis (2004).

[18] Y. Yamamura and H. Tawara, Energy dependence of ion-induced sputtering yields from monatomic solids at normal incidence, Atomic Data and Nuclear Data Tables 62, 149 (1996).

[19] R. Rejoub, B. G. Lindsay, and R. F. Stebbings, Determination of the absolute partial and total cross sections for electron-impact ionization of the rare gases, Phys. Rev. A 65, 042713 (2002).

[20] V. V. Zhurin, Industrial Ion Sources (John Wiley \& Sons, 2011).

[21] B. Nichols and F. C. Witteborn, Measurements of resonant charge exchange cross sections in nitrogen and argon between 0.5 and 17 ev, Nasa Technical Note , 1 (1966).

[22] D. I. Astakhov, W. J. Goedheer, C. J. Lee, V. V. Ivanov, V. M. Krivtsun, A. I. Zotovich, S. M. Zyryanov, D. V. Lopaev, and F. Bijkerk, Plasma probe characteristics in low density hydrogen pulsed plasmas, Plasma Sources Science and Technology 24, 055018 (2015).

[23] D. Van Vechten, G. Hubler, and E. Donovan, Characterization of a $3 \mathrm{~cm}$ Kaufman ion source with nitrogen feed gas, Vacuum 36, 841 (1986).

[24] C. Lee, D. B. Graves, M. A. Lieberman, and D. W. Hess, Global model of plasma chemistry in a high density oxygen discharge, Journal of The Electrochemical Society 141, 1546 (1994), http://jes.ecsdl.org/content/141/6/1546.full.pdf + html .

[25] F. Pontiga, C. Soria, A. Castellanos, and J. D. Skalny, A study of ozone generation by negative corona discharge through different plasma chemistry models, Ozone: Science \& Engineering 24, 447 (2002), https://doi.org/10.1080/01919510208901634 .

[26] U. Fantz, Basics of plasma spectroscopy, Plasma Sources Science and Technology 15, S137 (2006). 
[27] J. Benedikt, A. Hecimovic, D. Ellerweg, and A. von Keudell, Quadrupole mass spectrometry of reactive plasmas, Journal of Physics D: Applied Physics 45, 403001 (2012).

[28] T. van de Ven, Ion fluxes towards surfaces exposed to EUV-induced plasmas, Ph.D. thesis, Department of Applied Physics (2018), proefschrift.

[29] Y. Yao, Z. Hargitai, M. Albert, R. Albridge, a. Barnes, J. Gilligan, B. Pratt Ferguson, G. Lüpke, V. Gordon, N. Tolk, J. Tully, G. Betz, and W. Husinsky, New Molecular Collisional Interaction Effect in Low-Energy Sputtering, Physical Review Letters 81, 550 (1998).

[30] T. Kalvas, O. Tarvainen, T. Ropponen, O. Steczkiewicz, J. Ärje, and H. Clark, Ibsimu: A three-dimensional simulation software for charged particle optics, Review of Scientific Instruments 81, $02 \mathrm{~B} 703$ (2010), https://doi.org/10.1063/1.3258608 .

[31] W. Möller, W. Eckstein, and J. P. Biersack, Tridyn-binary collision simulation of atomic collisions and dynamic composition changes in solids, Computer Physics Communications 51, 355 (1988).

[32] R. Kersevan and J.-L. Pons, Introduction to molflowt: New graphical processing unit-based monte carlo code for simulating molecular flows and for calculating angular coefficients in the compute unified device architecture environment, Journal of Vacuum Science \& Technology A 27, 1017 (2009), https://doi.org/10.1116/1.3153280 .

[33] M. Lieberman and A. Lichtenberg, Basic plasma equations and equilibrium, in Principles of Plasma Discharges and Materials Processing (John Wiley \& Sons, Ltd, 2005) Chap. 2, pp. 23-42, https://onlinelibrary.wiley.com/doi/pdf/10.1002/0471724254.ch2 .

[34] D. Lopaev, private communication (2017). 

(a)

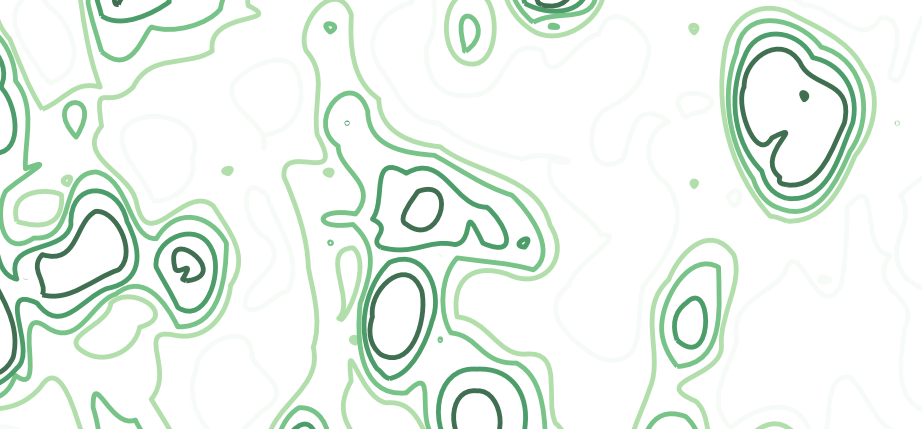

0

(c) 00

(a)

$(3) \int^{2}$

(2) (2)

0.250

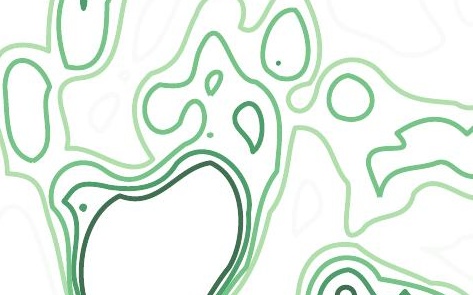

))

- 40

(a) (D)

(.) (0.)

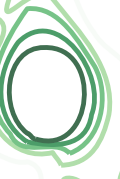

(i)

(a) 0

今0 (0)

a. (a)

0

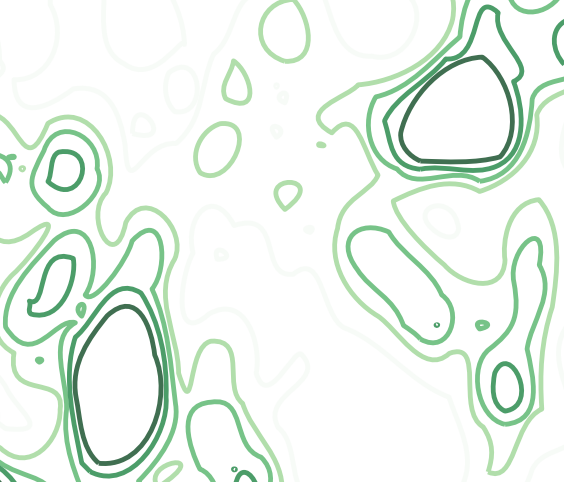

2(0.0 0

$\frac{5}{5}$

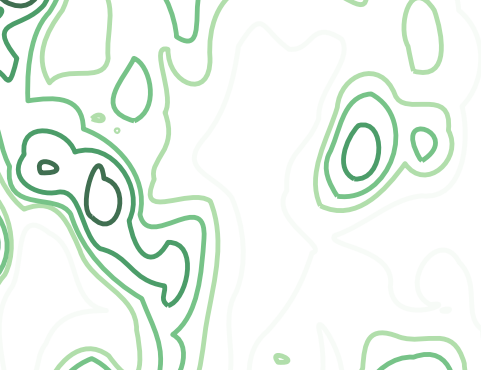

(0).
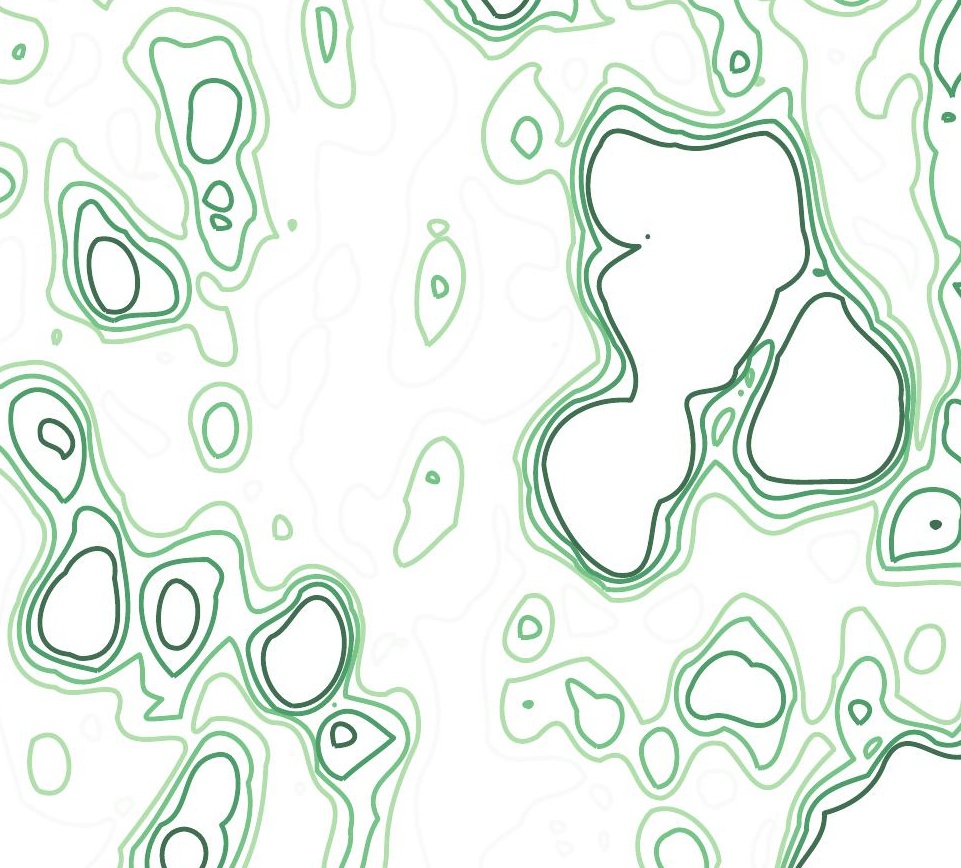

3

ion

(1) 0.0

(e)

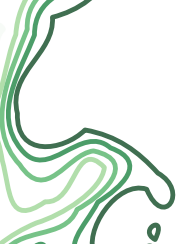

(c) (2) 8

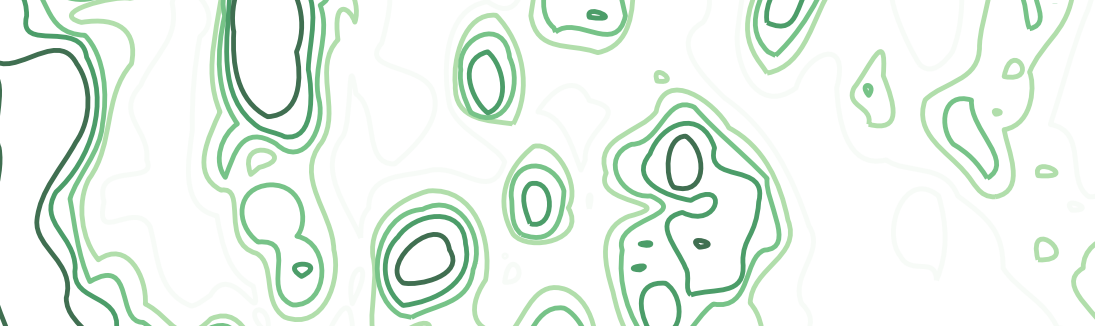

(0)

(0)

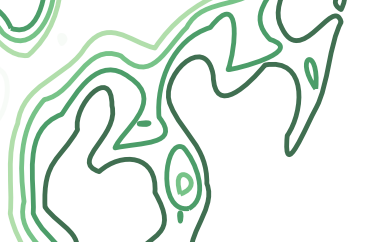




\section{3}

NEAR-THRESHOLD SPUTTER YIELDS Ruthenium: $\mathrm{Ar}^{+}$AND $\mathbf{N}_{2}^{+}$

Beginnings are always delightful, the threshold is the place to pause.

Goethe translated to english from Wilhelm Meister's Apprenticeship

Ion surface interactions near sputter-threshold are of interest for various plasma facing materials. We report experimental determination of sputter yields for ruthenium films grown on a quartz crystal microbalance and exposed to $\mathrm{Ar}^{+}$and $\mathrm{N}_{2}^{+}$ ions in the energy range of 50-300 eV. Comparison to semi-empirical models shows agreement to previously reported yields for argon bombardment. In the case of nitrogen, the Yamamura model was modified to account for molecular effects and the yields are found to be between extremes of rigid and non-rigid molecular approximations proposed by Yao. Ex-situ XPS measurements revealed implantation of nitrogen in the ruthenium film after exposure to nitrogen ions. The discrepancy between the models and experimental results for $\mathrm{N}_{2}^{+}$bombardment is explained by an increase in the surface binding energy of the target leading to a chemically reduced sputter yield. 


\subsection{Introduction}

Terrestrial plasmas occur in a wide range of densities and temperatures. High temperature, dense plasmas in fusion reactors [1], diffuse plasmas induced by extreme ultraviolet radiation [2] and dense low pressure plasmas in laboratory ion sources and for electric propulsion [3]. Plasma facing materials (PFMs), such as divertors in fusion reactors are bombarded by hydrogen and deuterium ion species [4], ion lensing systems for electric propulsion technology deteriorate under xenon ion impingement and optics in next generation lithography applications face diffuse hydrogen and nitrogen plasmas $[5,6]$. There is, thus, a need to quantify the damage incurred by various PFMs. Sputter yields provide a reasonable estimate of optic material damage due to mass loss when facing diffuse low energy plasmas. However, for reactive ions, chemical effects become dominant near the sputter threshold and need to be accounted for.

While experimental data on sputter yields exist for transition metals under nitrogen ion impingement [7-10], ruthenium is not well studied. Semi-empirical models account for sputtering by assuming a uniform potential barrier at the surface called the surface binding energy (SBE). This is approximated to the enthalpy of sublimation for most targets. Chemical effects such as compound formation during the sputter process would lead to changes in the SBE. Ruthenium nitride $\left(\mathrm{RuN}_{\mathrm{x}}\right)$ is predicted to have a positive enthalpy of formation [11]. The formation energy barrier is overcome by the incidence of energetic nitrogen ions or radicals either by magnetron sputtering [12, 13] plasmas or pulsed lasers [14]. The formation of $\mathrm{RuN}_{\mathrm{x}}$ during sputter measurements would modify the SBE for such combinations involving reactive species leading to larger deviations in model predictions.

This paper serves as beginning to a series of experiments to study the interaction of nitrogen and other reactive ion species to a variety of PFMs. In this report, we consider the sputtering of ruthenium under argon and nitrogen bombardment and ascertain the validity of semi-empirical models and their approximation of the SBE for compound formation.

\subsection{Experimental Details}

The setup of the exposure facility has been described in detail in a previous publication [15]. A commercial $15 \mathrm{~cm}$ DC Kaufman-type ion source with a molybdenum three-grid lensing system was used for generating mono-energetic ion beams of argon and nitrogen with energies down to $50 \mathrm{eV}$ at current densities of $100 \mu \mathrm{A} / \mathrm{cm}^{2}$. A residual gas analyzer (RGA) was used to measure the background gases in the system, which were dominated by water vapor at a level of $10^{-9}$ mbar. A faraday cup that also functions as a retarding field energy analyzer (FC/RFEA) with an entrance aperture of $1.5 \mathrm{~mm}$ diameter was retrofitted onto the source. A $300 \mathrm{~nm}$ ruthenium film was deposited on an AT-cut quartz crystal microbalance (QCM) by magnetron sputtering prior to installing it on the source-FC+RFEA assembly. The roughness of the film was not determined. It was shown previously [15] that the sputter yields obtained for a film on a quartz crystal microbalance were similar to the ones determined by exposure of an atomically smooth film on a witness sample. 
The QCM was placed at the same radial distance from the center of the source axis as the FC+RFEA. This allowed for the measurement of the current variations throughout the exposure of the ruthenium film to the ions.

The source was operated at a discharge voltage $\left(V_{d}\right)$ of $50 \mathrm{~V}$ and $75 \mathrm{~V}$ for argon and nitrogen, respectively. The beam was calibrated using the $\mathrm{FC}+\mathrm{RFEA}$ and the ion energy distributions after a Gaussian fit were found to be mono-energetic with an energy spread between 5-9 eV over the energy range of interest. Analyses of beam contents from similar Kaufman ion sources provide expectation values for argon and nitrogen: At a $V_{d}$ of $60 \mathrm{~V}$, the concentration of doubly charged ions in the argon plasma is less than 1\% [16]. For nitrogen, experiments have shown [17] that while a wide range of parameters influences the composition of the beam, major fractions in current result due to $\mathrm{N}^{+}$and $\mathrm{N}_{2}^{+}$. Based on their analysis we expect $\mathrm{N}_{2}^{+}$ions making up 80-90\% of the beam current for our working conditions with the remainder being $\mathrm{N}^{+}$. Performing a weighted average on the yields predicted by the Yamamura model (equation 3.1), for the various species present in the beam, we estimate the deviation of the yield to an upper limit of $18 \%$ for nitrogen and $9 \%$ for argon at $50 \mathrm{eV}$ due to presence of atomic ions and doubly charged ions respectively. We also estimate a reduction of current density measured at the FC due to resonant neutralization processes [18] up to 2 and 5 percent for argon and nitrogen respectively at a working distance of $7 \mathrm{~cm}$.

Data acquisition of the currents and energy measurements were carried out via a Keithley 2100 micro-ammeter controlled by a MATLAB script running on a personal computer. The QCM frequency response and the ion source were controlled through a LABVIEW program. The frequency response from the QCM was converted to a thickness value using the Z-match method [15, 19]. Data between the distortions induced by thermal shock at the start and end of the ion irradiation was used to calculate rate of thickness loss (Figure 3.1) and subsequently, the sputter yields.

The current fluctuations over the dose were estimated to be under $5 \%$ for the energies of interest. The exposure energies were selected at random to avoid systematic errors. In both experiments, the surface oxide was sputter cleaned with 300 $\mathrm{eV} \mathrm{Ar}^{+}$. Additionally, for nitrogen exposures, the surface was treated with $300 \mathrm{eV}$ $\mathrm{Ar}^{+}$after each exposure to obtain a clean ruthenium surface prior to each nitrogen ion exposure. Each experiment was carried out until a dose of $1 \times 10^{18}$ ions $/ \mathrm{cm}^{2}$ was irradiated on the surface to ensure sputter yield is measured at steady state and enough statistics are available for the low energy exposures.

At the end of the experiment, the ruthenium coated QCM sample was transferred to an X-Ray Photoelectron Spectroscopy (XPS) setup for characterization. The sample remained in atmosphere for two days before it could be characterized. XPS measurements were done for the ruthenium film at the last energy of irradiation: 60 eV nitrogen ions, using a Thermo-Fisher Theta probe instrument with a beam spot size of $400 \mu \mathrm{m}$ diameter at an angular range of $\pm 30^{\circ}$ around an average take-off angle of $53^{\circ}$ with respect to the surface normal. 


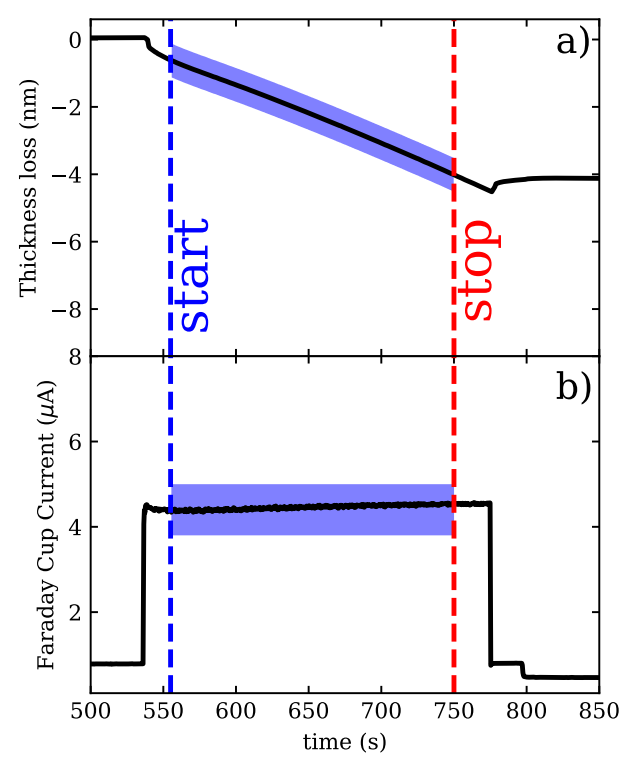

Figure 3.1: Measurement of (a) thickness loss and (b) ion current made simultaneously with a quartz crystal microbalance and a Faraday cup. The start and stop lines (representative purposes) denote the data used for determining the sputter yield.

\subsection{Theoretical Models}

We choose the Yamamura [20] model for comparison with experimental data because of its extensive usage, and Eckstein's [21] formula for its implementation simplicity. Both build upon Sigmund's original sputter formula to take into account the presence of a sputter threshold. Eckstein's model provided modifications over the Yamamura model by correcting the steep rise of yields above the threshold energy otherwise predicted by the Yamamura model. This was shown to agree well for inert gas ions and self sputtering [22]. It is applied for a wide range of ion-target combinations [23, 24]. The Yamamura model is described as:

$$
Y(E)=0.042 \frac{Q\left(Z_{2}\right) \alpha^{*}\left(M_{1}, M_{2}\right)}{U_{s}} \frac{S_{n}(E)}{1+\Gamma k_{e} \epsilon^{0.3}}\left(1-\sqrt{\frac{E_{t h}}{E}}\right)^{s}
$$

where $M_{1}$ is the mass of the ion, $M_{2}$ is the mass of the target atom; $E_{t h}, s$ and $Q$ are fitting parameters. $E_{t h}$ is the threshold energy for sputtering. $S_{n}$ is the nuclear stopping power of the target element and the parameter $\Gamma$ factors in the contribution of reflected ions to the recoil cascade and $U_{s}$ is the SBE which is usually approximated to the sublimation energy of the target material. And Eckstein's formula takes the form: 


$$
Y(E)=q s_{n}^{K r C}\left(\epsilon_{L}\right) \frac{\left(\frac{E}{E_{t h}}-1\right)^{\mu}}{\frac{\lambda}{w\left(\epsilon_{L}\right)}+\left(\frac{E}{E_{t h}}-1\right)^{\mu}}
$$

where $q, E_{t h}, \mu$ and $\lambda$ are used as fitting parameters. $s_{n}^{K r C}$ is the reduced nuclear stopping power based on the $\mathrm{Kr}-\mathrm{C}$ interaction potential which is a function of the reduced ion energy, $\epsilon_{L} . \epsilon_{L}$ scales the ion energy with the Lindhard screening length and the masses of the interacting species. Refer to [22] for details. In both cases, $Y$ is the theoretical sputter yield and $E$ is the energy of the incident ion.

While these formulae give a reasonable estimate to the sputter yields for noble gas ions, their accuracy for nitrogen is unreliable due to poor comparison to experimental data. The models have, thus far not been compared with the present ion-target combination. For reactive species, their predictions can be considered a 'static' case, i.e., the yield observed when the first ion hits the target. This would lead to an over-estimation when compound formation occurs, and an underestimation for chemical sputtering processes. The SBE would deviate dynamically as the surface composition and ion fluence changes. Compound formation or accumulation of ions would affect the sputter yield $[9,10]$. The models are limited in their ability to account for changes in composition of target materials due to implantation or compound formation. The Yamamura model provides a surface binding energy term to include a surface potential for sputtering. In order to assess chemical changes by the ion species, a fit to the Yamamura model was performed where $U_{s}$ was added as a fit parameter.

\subsection{Results and Discussion}

Figure 3.2a shows the sputter yields obtained for ruthenium under argon bombardment. The yields obtained for argon are consistent with data reported by Laegreid [25] for polycrystalline rods and by $\mathrm{Wu}$ [15] for e-beam deposited ruthenium. The deviations in the data can be accounted by the changes in film density and roughness in the targets studied [26, 27]. A Markov Chain Monte Carlo (MCMC) code [28] was used to calculate the posterior probabilities of the fitting parameters and only the present dataset was used for ease of comparison with the nitrogen data and prevent any bias by the additional datasets. The fit result is plotted in Figure 3.2a. The obtained best-fit value for $U_{s}$ is $7.5 \pm 2.8 \mathrm{eV}$ which is similar to the sublimation energy of $6.7 \mathrm{eV}$ used for the initial Yamamura prediction. A sputter threshold of $38.4 \pm 1.4 \mathrm{eV}$ obtained from the fit is found to be larger than previous work by $\sim 15 \%$.

Sputter yields from nitrogen bombardment of ruthenium compared to the Yamamura model are shown in Figure 3.2b. The expected sputtering by molecular species depends on an effective mass, $m^{*}$, that lies between two extremes [29] $M_{1} \leq$ $m^{*} \leq 2 M_{1}$ for when the molecule acts as a rigid ensemble with twice the mass of a single atom $\left(2 M_{1}\right)$ or as two individual atoms $\left(M_{1}\right)$ with half of the original energy. The experimental yields in an ideal situation of no chemical interaction, would lie between these two limiting cases. This molecular effect is dominant for when the 

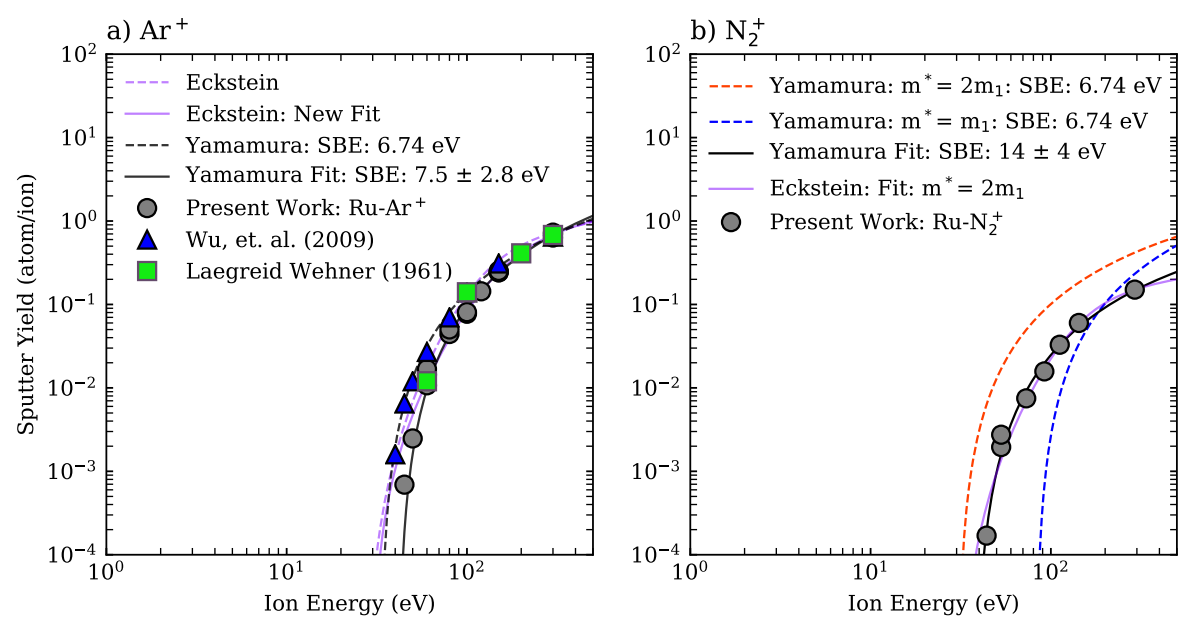

Figure 3.2: a) Sputter yields obtained from QCM response (circles) compared to reports from $\mathrm{Wu}$ (triangles) and Laegreid (squares). The Yamamura (solid black) and Eckstein (solid purple) models with best-fit parameters from Table 3.1 are plotted. (b) For $\mathrm{N}_{2}^{+} 2$ yields, the Yamamura model for a rigid $\left(\mathrm{m}^{*}=2 \mathrm{~m}\right)$ and a non-rigid $\left(m^{*}=m\right)$ molecule are plotted and compared to a new fit with a modified surface binding energy; New parameters obtained for Eckstein's formula (purple) are also plotted which show marked deviations near the sputter threshold.

vibrational frequency of the molecule is comparable to the interaction time of the collision.

From Yao's assumptions [29] of ion-surface interaction times, the molecular effect would occur below $250 \mathrm{eV}$ for nitrogen ions. For high energies, atomic and molecular species would sputter equally efficiently. For the comparison with experimental data of nitrogen, parameters in equation 3.1 were modified to account for the two mass limits and depicted in Figure 3.2b. Using the same procedure for fitting the data as for argon, and using the model with $m^{*}=2 M_{1}, U_{s}$ is found to be $14 \pm 4 \mathrm{eV}$. $Q$ and $s$ remain the same within error for both ion species. A sputter threshold of $37 \pm 2 \mathrm{eV}$ was determined from the fit.

Eckstein provides a compendium of tabulated values in [24] for the fitting parameters for a wide range of ion and target combinations. However, to our knowledge, no reported best-fit parameter values for a ruthenium and nitrogen combination are available. We provide an estimate to the model parameters using the MCMC approach. The results are summarized in Table 3.1.

Chemical interaction between the ruthenium and nitrogen is evident from the post exposure XPS as shown in Figure 3.3. The ruthenium 3d spectrum of the QCM exposed to $60 \mathrm{eV}$ nitrogen was compared to a clean ruthenium reference after a Shirley background subtraction. The contribution to the broadening of the metal peaks by oxide formation from an exposure to atmosphere and the nitride formed by the ion exposure experiment are difficult to quantify. The lack of a significant peak shift of the Ru3d peaks indicates that the sample remains mostly metallic, consistent with transition metal nitrides [13]. 


\begin{tabular}{|c|c|c|c|c|c|c|c|c|c|}
\hline \multirow[t]{2}{*}{ Ion } & & \multicolumn{4}{|c|}{ Yamamura Model } & \multicolumn{4}{|c|}{ Eckstein Model } \\
\hline & & $Q$ & $s$ & $\begin{array}{c}U_{s} \\
(\mathrm{eV})\end{array}$ & $\begin{array}{l}E_{t h} \\
(\mathrm{eV})\end{array}$ & $\lambda$ & $q$ & $\mu$ & $\begin{array}{c}E_{t h} \\
(\mathrm{eV})\end{array}$ \\
\hline \multirow[t]{3}{*}{$A r^{+}$} & & $1.31^{a}$ & $2.5^{a}$ & $6.74^{a}$ & $33^{a}$ & $0.19^{b}$ & $6.84^{b}$ & $2.20^{b}$ & $27.47^{6}$ \\
\hline & MCMC & 1.86 & 2.73 & 7.5 & 38.4 & 0.28 & 8.4 & 1.88 & 26.2 \\
\hline & error & \pm 0.68 & \pm 0.18 & \pm 2.8 & \pm 1.4 & \pm 0.14 & \pm 1.14 & \pm 0.35 & \pm 5.6 \\
\hline \multirow[t]{2}{*}{$N_{2}^{+}$} & & $1.31^{c}$ & $2.5^{c}$ & $6.74^{c}$ & $28^{c}$ & - & - & - & - \\
\hline & $\begin{array}{l}\text { MCMC } \\
\text { error }\end{array}$ & $\begin{array}{c}1.14 \\
\pm 0.42\end{array}$ & $\begin{array}{c}2.75 \\
\pm 0.17\end{array}$ & $\begin{array}{l}14 \\
\pm 4\end{array}$ & $\begin{array}{l}37.2 \\
\pm 2.2\end{array}$ & $\begin{array}{c}0.31 \\
\pm 0.13\end{array}$ & $\begin{array}{c}1.01 \\
\pm 0.03\end{array}$ & $\begin{array}{c}2.13 \\
\pm 0.17\end{array}$ & $\begin{array}{r}30.0 \\
\pm 4.8 \\
\end{array}$ \\
\hline
\end{tabular}

$\overline{{ }^{a} \text { Reference: [15] }}$

${ }^{b}$ Reference: [24]

${ }^{c}$ Reference: [20]

Table 3.1: Fit parameters with errors obtained from Markov Chain Monte Carlo code after 35,000 steps including a burn-in period of 5000. See [28] for details of the algorithm.

The O1s peak for air exposed nitrides shares similar difficulties as the contributions from the oxy-nitride and $\mathrm{O}-\mathrm{H}$ groups from adsorbed water and hydroxides are challenging to deconvolve. The peaks before and after exposure are shown in Figure 3.3c for qualitative comparison. The oxygen before exposure contained a metallic oxide at around $529 \mathrm{eV}$ which is a dominating contribution after exposure to nitrogen and subsequently atmosphere. The quantification of the nitride and oxynitride is therefore limited to the N1s spectra.

Two components of the N1s have been identified, one at $397.3 \mathrm{eV}$ corresponding to the nitride and $398.8 \mathrm{eV}$ which is characteristic for an oxynitride for transition metal nitrides exposed to atmosphere $[13,30]$. The area under the N1s peak corresponded to 18 atomic percent of nitrogen in the measured sample volume, implying implantation of nitrogen within the target. This is consistent with reports that $\mathrm{RuN}_{\mathrm{x}}$, even though thermodynamically unfavorable, is formed by reactive magnetron sputtering [12].

\subsection{Conclusions}

In summary, we report sputter yields for ruthenium under argon and nitrogen ion bombardment. Argon shows agreement to the Yamamura model with discrepancies compared to literature that possibly originate from density and roughness variations in the targets analyzed. Theoretical yields for nitrogen ions on $\mathrm{Ru}$ provide boundaries for expectation values, but over-estimate experimental yields for a rigid molecule approximation. SBE is approximated by sublimation energies for modeling purposes. Transition metals and their nitrides show variation in the sublimation energy as can be seen in case of $\mathrm{Zr}$ [31] where the experimentally determined sublimation energy of the metal is $2.7 \mathrm{eV}$ lower than that of the nitride. The approximation of the surface binding energy to the sublimation energy would then be inappropriate for a reactive ion bombarding a metal. Using the $\operatorname{SBE}\left(U_{s}\right)$ as a fit 


\section{b) N1s}

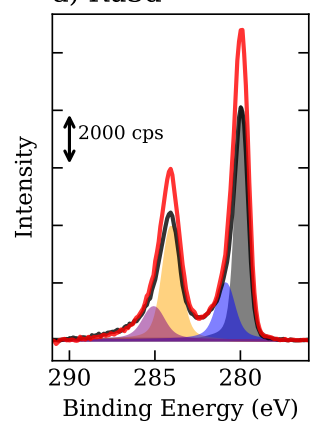

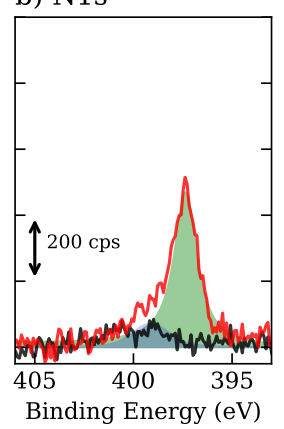

c) $\mathrm{O} 1 \mathrm{~s}$

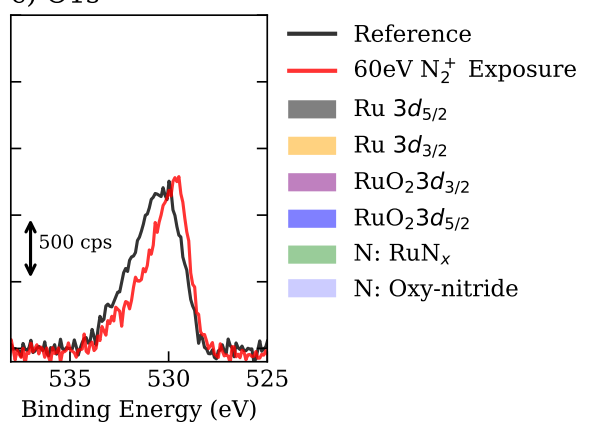

Figure 3.3: XPS Spectra of Ru coated QCM both measured after exposure to air: with reference (bottom) and $60 \mathrm{eV} \mathrm{N} N_{2}^{+}$exposed (top) samples. The Ru3d peaks (a) remain mostly metallic while an N1s (b) peak appears post exposure. See text for details.

parameter showed a change of $U_{s}$ for ruthenium from argon exposure to nitrogen exposure by $1.8 \mathrm{x}$. We hypothesize that the chemical interaction between nitrogen and ruthenium, as observed by XPS, leads to formation of ruthenium nitride and a change in the SBE.

In the next chapter we shall explore the retention of nitrogen in ruthenium as a function of incident ion energy. We shall further compare its behavior to other transition metals. 


\section{References}

[1] C. Watts, V. Udintsev, P. Andrew, G. Vayakis, M. Van Zeeland, D. Brower, R. Feder, E. Mukhin, and S. Tolstyakov, Electron density measurements in the ITER fusion plasma, Nuclear Instruments and Methods in Physics Research, Section A: Accelerators, Spectrometers, Detectors and Associated Equipment 720, 7 (2013).

[2] T. Van De Ven, P. Reefman, C. De Meijere, R. Van Der Horst, M. Van Kampen, V. Banine, and J. Beckers, Ion energy distributions in highly transient euv induced plasma in hydrogen, Journal of Applied Physics 123, 063301 (2018).

[3] D. A. Herman and A. D. Gallimore, Comparison of Discharge Plasma Parameters in a 30-cm NSTAR Type Ion Engine with and without Beam Extraction, 39th AIAA/ASME/SAE/ASEE Joint Propulsion Conference \& Exhibit, AIAA 2003 (2003).

[4] J. Roth, E. Tsitrone, A. Loarte, T. Loarer, G. Counsell, R. Neu, V. Philipps, S. Brezinsek, M. Lehnen, P. Coad, C. Grisolia, K. Schmid, K. Krieger, A. Kallenbach, B. Lipschultz, R. Doerner, R. Causey, V. Alimov, W. Shu, O. Ogorodnikova, A. Kirschner, G. Federici, and A. Kukushkin, Recent analysis of key plasma wall interactions issues for ITER, Journal of Nuclear Materials 390-391, 1 (2009).

[5] M. H. L. Van Der Velden, W. J. M. Brok, J. J. A. M. Van Der Mullen, W. J. Goedheer, and V. Banine, Particle-in-cell Monte Carlo simulations of an extreme ultraviolet radiation driven plasma, Physical Review E - Statistical, Nonlinear, and Soft Matter Physics 73, 1 (2006).

[6] R. C. Wieggers, W. J. Goedheer, M. R. Akdim, F. Bijkerk, and P. A. Zegeling, A particle-in-cell plus Monte Carlo study of plasma-induced damage of normal incidence collector optics used in extreme ultraviolet lithography, Journal of Applied Physics 103 (2008), 10.1063/1.2829783.

[7] K. Dobes, P. Naderer, N. Lachaud, C. Eisenmenger-Sittner, and F. Aumayr, Sputtering of tungsten by $n+$ and $n 2+$ ions: investigations of molecular effects, Physica Scripta 2011, 014017 (2011).

[8] R. Ranjan, J. P. Allain, M. R. Hendricks, and D. N. Ruzic, Absolute sputtering yield of ti/ tin by ar $+/ n+$ at 400-700 ev, Journal of Vacuum Science \& Technology A 19, 1004 (2001).

[9] K. Schmid, A. Manhard, C. Linsmeier, A. Wiltner, T. Schwarz-Selinger, W. Jacob, and S. Maendl, Interaction of nitrogen plasmas with tungsten, Nuclear Fusion 50, 025006 (2010).

[10] G. Meisl, K. Schmid, O. Encke, T. Höschen, L. Gao, and C. Linsmeier, Implantation and erosion of nitrogen in tungsten, New Journal of Physics 16 (2014), 10.1088/1367-2630/16/9/093018. 
[11] Y. Zhang, L. Wu, B. Wan, Y. Lin, Q. Hu, Y. Zhao, R. Gao, Z. Li, J. Zhang, and H. Gou, Diverse ruthenium nitrides stabilized under pressure: A theoretical prediction, Scientific Reports 6, 1 (2016).

[12] E. Cattaruzza, G. Battaglin, P. Riello, D. Cristofori, and M. Tamisari, On the synthesis of a compound with positive enthalpy of formation: Zinc-blende-like RuN thin films obtained by rf-magnetron sputtering, Applied Surface Science 320, 863 (2014).

[13] J. H. Quintero, R. Ospina, A. Mello, D. Escobar, and E. Restrepo-Parra, Influence of nitrogen partial pressure on the microstructure and morphological properties of sputtered RuN coatings, Surface and Interface Analysis 49, 978 (2017).

[14] M. G. Moreno-Armenta, J. Diaz, A. Martinez-Ruiz, and G. Soto, Synthesis of cubic ruthenium nitride by reactive pulsed laser ablation, Journal of Physics and Chemistry of Solids 68, 1989 (2007).

[15] S. M. Wu, R. Van De Kruijs, E. Zoethout, and F. Bijkerk, Sputtering yields of Ru, Mo, and Si under low energy Ar+ bombardment, Journal of Applied Physics 106, 0 (2009).

[16] M. Zeuner, J. Meichsner, H. Neumann, F. Scholze, and F. Bigl, Design of ion energy distributions by a broad beam ion source, Journal of Applied Physics 80, 611 (1996).

[17] D. Van Vechten, G. Hubler, and E. Donovan, Characterization of a $3 \mathrm{~cm}$ Kaufman ion source with nitrogen feed gas, Vacuum 36, 841 (1986).

[18] V. V. Zhurin, Industrial Ion Sources (John Wiley \& Sons, 2011).

[19] A. Wajid, Improving the accuracy of a quartz crystal microbalance with automatic determination of acoustic impedance ratio, Review of Scientific Instruments 62, 2026 (1991).

[20] Y. Yamamura and H. Tawara, Energy dependence of ion-induced sputtering yields from monatomic solids at normal incidence, Atomic Data and Nuclear Data Tables 62, 149 (1996).

[21] W. Eckstein, Sputtering yields, Vacuum 82, 930 (2008).

[22] W. Eckstein and R. Preuss, New fit formulae for the sputtering yield, Journal of Nuclear Materials 320, 209 (2003).

[23] Z. Somogyvári, G. A. Langer, G. Erdélyi, and L. Balázs, Sputtering yields for low-energy Ar +- and Ne +-ion bombardment, Vacuum 86, 1979 (2012).

[24] W. Eckstein, Sputtering yields. in: Sputtering by particle bombardment: Experiments and computer calculations from threshold to mev energies, in Sputtering by Particle Bombardment: Experiments and Computer Calculations from Threshold to MeV Energies (Springer Berlin Heidelberg, Berlin, Heidelberg, 2007) pp. 33-187. 
[25] N. Laegreid and G. K. Wehner, Sputtering yields of metals for ar + and ne+ ions with energies from 50 to 600 ev, Journal of Applied Physics 32, 365 (1961).

[26] V. Shulga, The density effects in polycrystal sputtering, Nuclear Instruments and Methods in Physics Research Section B: Beam Interactions with Materials and Atoms 174, 77 (2001).

[27] M. A. Makeev and A. L. Barabási, Effect of surface morphology on the sputtering yields. II. Ion sputtering from rippled surfaces, Nuclear Instruments and Methods in Physics Research, Section B: Beam Interactions with Materials and Atoms 222, 335 (2004).

[28] D. Foreman-Mackey, D. W. Hogg, D. Lang, and J. Goodman, emcee: The mcmc hammer, Publications of the Astronomical Society of the Pacific 125, 306-312 (2013).

[29] Y. Yao, Z. Hargitai, M. Albert, R. Albridge, a. Barnes, J. Gilligan, B. Pratt Ferguson, G. Lüpke, V. Gordon, N. Tolk, J. Tully, G. Betz, and W. Husinsky, New Molecular Collisional Interaction Effect in Low-Energy Sputtering, Physical Review Letters 81, 550 (1998).

[30] Y. Kamiura, K. Umezawa, Y. Teraoka, and A. Yoshigoe, Characterization of polycrystalline tungsten surfaces irradiated with nitrogen ions by x-ray photoelectron spectroscopy, Materials Transactions 57, 1609 (2016).

[31] K. A. Gingerich, Gaseous Metal Nitrides. II. The Dissociation Energy, Heat of Sublimation, and Heat of Formation of Zirconium Mononitride, The Journal of Chemical Physics 49, 14 (1968). 


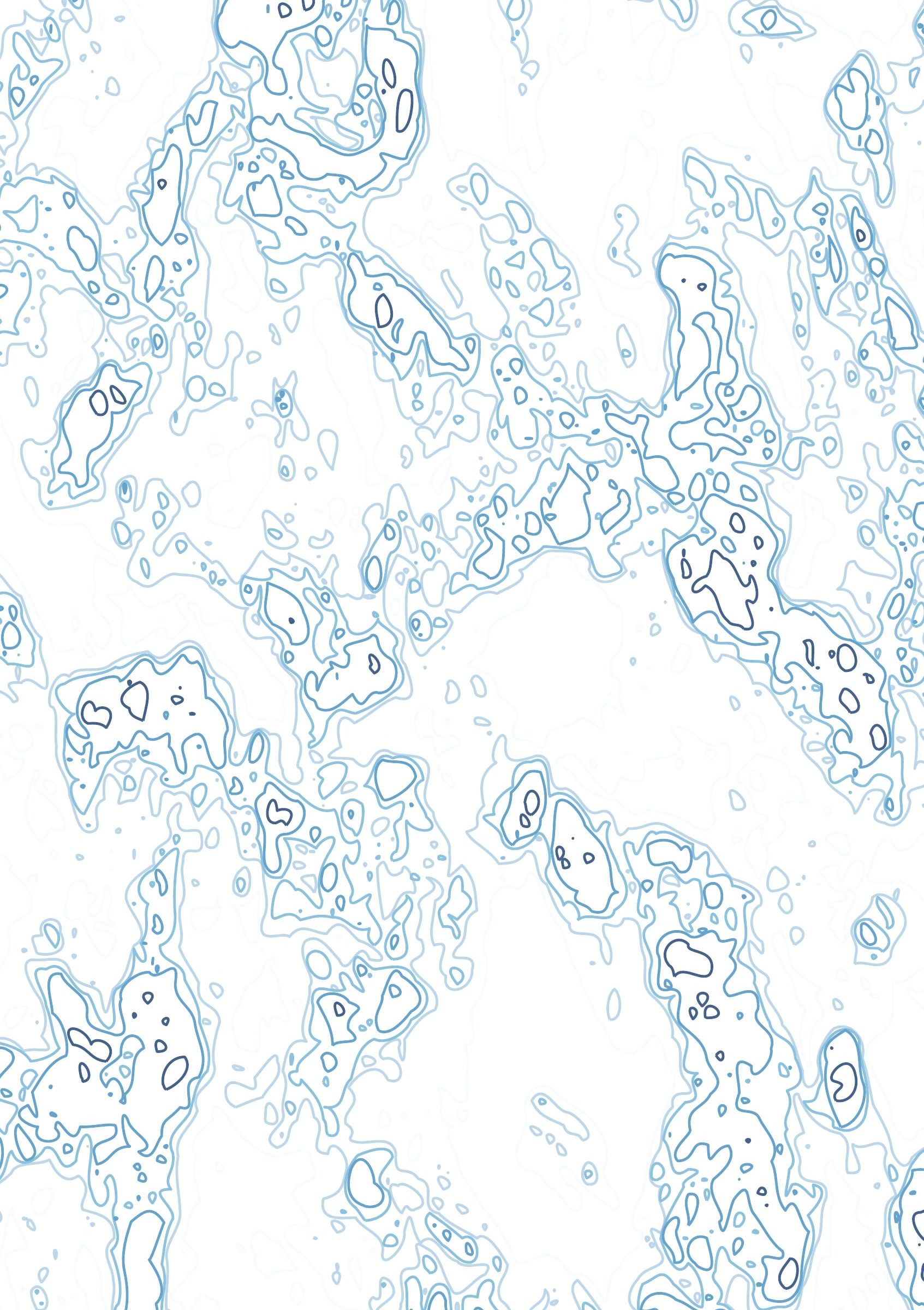




\section{4}

\section{SPUTTERING AND Nitridation:}

\section{XPS RECONSTRUCTIONS}

Gentlemen, we can rebuild him. We have the technology.

The Six Million Dollar Man

Transition metal surfaces exposed to low-energy reactive ions undergo dynamic changes in composition and density due to implantation and compound formation. We report measurements of nitrogen ion induced sputter yields for transition metals relevant to fusion and optics applications. Thin films of molybdenum, ruthenium, palladium and tungsten are bombarded by nitrogen ions of kinetic energies in the range of $50-500 \mathrm{eV}$ at steady state fluences $\left(1 \times 10^{18}\right.$ ions $\left./ \mathrm{cm}^{2}\right)$. Measured sputter yields are explained through energy and momentum transfer under the binary collision approximation using the Monte Carlo code TRIDYN. X-Ray Photoelectron spectroscopy (XPS) studies showed the nitrogen content in the films at the end of ion exposure is independent of incoming ion energy. This occurs due to competing implantation and preferential surface nitrogen sputtering processes within the XPS probing depth. All metals investigated showed evidence of a nitride formed due to energetic nitrogen impact. The combination of XPS and TRIDYN simulations were applied to extract effective reaction cross-sections for each metal. 


\subsection{Introduction}

The interaction of a plasma with a metal surface is a common phenomenon in a multitude of scenarios: solar winds interact with satellite shielding [1]; particles in accelerators collide with electrostatic optics [2]; divertors in fusion reactors face high ion fluxes [3]; diffuse plasmas in extreme ultraviolet (XUV) lithography may interact with reflective optics [4-6]; magnetically confined plasmas in sputter deposition systems bombard metallic targets [7]. In nearly all cases, with the exception of sputter deposition, the interaction is unwanted and possibly damaging to the metal surface. Damage can manifest in many forms, some of which as: a loss of material; modification of surface topography; change of surface or sub-surface composition compromising functionality. Understanding the mechanisms of damage is therefore necessary to limit aforementioned changes and extend operational time.

The interaction of low energy nitrogen plasmas with transition metal surfaces is of academic and practical interest. Sputter damage [3] and ion retention [8] studies in plasma facing materials (PFM) such as tungsten (W) have proven helpful in assessing the viability of using nitrogen as a coolant in fusion plasmas. Liquid lithium is proposed as a PFM with molybdenum (Mo) as a substrate which would potentially be exposed to fusion plasmas as well [9]. Ruthenium ( $\mathrm{Ru}$ ) coatings in XUV applications may face plasmas and while sputtering has been investigated $[10,11]$, retention is poorly understood. Palladium has been studied as well for PFM applications concerning hydrogen and tritium recycling through recombination and desorption $[12,13]$. The use of a nitrogen seed gas would require studies of palladium under nitrogen ion bombardment.

Currently available data on low energy nitrogen sputtering and retention is limited to energies above $0.5 \mathrm{keV}$. We aim to extend this datasets to more operationally relevant energies near the sputter threshold. In this paper, sputter yields for Mo, $\mathrm{W}, \mathrm{Pd}$ and $\mathrm{Ru}$ atoms are measured down to $50 \mathrm{eV}$. The lower limit is dictated by experimental limitations of a low flux below $50 \mathrm{eV}$ which extends experimental times to unreasonable durations. Below $50 \mathrm{eV}$, where sputtering does not dominate, deposition of adsorbent species and grid material from the ion source places further restrictions on lowest energy.

Near the sputter threshold, where sticking coefficients dominate over scattering coefficients, chemical effects begin to play a role in the evolution of collision cascades. Evidence of a chemical component involving nitride formation during the sputtering process is presented through X-Ray photoelectron spectroscopy (XPS) analysis of ion irradiated thin films. The amount of retained nitrogen is compared among elements through corroboration of experimental measurements to Monte Carlo simulations. The retained nitrogen from XPS measurements is then used to determine effective reaction cross-sections for each element.

\subsection{Experimental methods}

Experiments studying composition changes and measuring sputter efficiency place strict constraints on: the vacuum system, demanding ultra-high vacuum with bakeout; ion generation, demanding energy, composition and flux calibration; and metrol- 
ogy, requiring depth sensitivity comparable to the ion affected region. We describe in the following subsections, the tools available, restrictions imposed and countermeasures applied.

\subsubsection{Ion exposure facility}

The exposure facility has been described in previous publications [10, 11]. In short, a thin film deposition system equipped with magnetrons for sputter deposition and a $15 \mathrm{~cm}$ DC Kaufman ion source (Veeco Instruments) was used for the experiments. The base pressure was $1 \times 10^{-8}$ mbar after bake-out dominated by water vapor. Samples of relatively thick $(80-100 \mathrm{~nm})$ films of $\mathrm{Pd}, \mathrm{Ru}$, Mo and $\mathrm{W}$ were deposited on single crystal silicon substrates for investigating composition. Additionally, $400 \mathrm{~nm}$ of each metal was deposited on separate quartz crystal microbalances (QCM) for insitu thickness loss measurements. A Faraday cup with an aperture of $1.5 \mathrm{~mm}$ along with a sample holder were mounted $7 \mathrm{~cm}$ in front of the ion source at the same radial distance off the ion source radial axis. The sample holder allowed for simultaneously exposing four samples while monitoring the ion flux during measurements. The faraday cup can also be used as a retarding field energy analyzer (FC+RFEA) and was used to measure the ion beam energies prior to the experiments. For all employed energies, the ion source produces a mono-energetic beam with a full-width at half maximum of $5-9 \mathrm{eV}$.

The ion source was operated at a flow of $8 \mathrm{sccm}$ of nitrogen and a discharge voltage of $75 \mathrm{~V}$. Direct measurement of the ion beam composition was not possible, however, literature reports on a similar DC Kaufman source [14] show the composition is dominated by $N_{2}^{+}$and $N^{+}$ions and is sensitive to the particular operating parameters such as pressure, beam current, discharge voltage and accelerator voltage. The pressure ranges reported in [14] are comparable to ours and at low energies the reported beam composition is independent of pressure. For other parameters, the variation in $\left[\mathrm{N}^{+}\right] /\left[\mathrm{N}_{2}^{+}\right]$is between $1 / 12-1 / 5$ corresponding to a $\mathrm{N}^{+}$content varying between $10-20 \%$ with $80-90 \% \mathrm{~N}_{2}^{+}$. The loss in beam current due to charge-exchange collisions of fast ions with background gas between the source exit and the entrance of the FC+RFEA is estimated to be $\sim 10 \%$ from the resonant neutralization cross-sections [15] extrapolated to our working energy range and $1 \times 10^{-4}$ mbar operating pressures. Neutralization thus leads to an under-estimation of the incident flux of energetic particles and an over-estimation of sputter yields. Neutralization loss is factored into errors of flux determination and subsequently propagated to the yield estimation. The uncertainties from the measurement of material loss by the QCM however, dominate over the errors from energetic neutrals.

Experiments for measuring sputter yields were carried out as per the method described previously [11]. The film surfaces on QCMs were sputter-cleaned with 300 $\mathrm{eV} \mathrm{Ar}^{+}$before nitrogen exposure to remove surface oxides and adsorbed contaminants. It was subsequently exposed to a nitrogen ion beam with a set ion energy. The frequency response from the QCM was monitored and converted to a thickness using the Z-match method [16]. The ion flux was simultaneously measured using the $\mathrm{FC}+\mathrm{RFEA}$. As the QCM allowed for multiple variations in energy within the 
same experiment, an $\mathrm{Ar}^{+}$cleaning step was performed between each nitrogen ion exposure. The energy variations were randomly chosen to avoid systematic errors. Sample and QCM experiments were carried out until an ion fluence of the order of $1 \times 10^{18}$ ions $/ \mathrm{cm}^{2}$ where the quartz response to etching was linear (steady-state). A steady-state fluence was chosen to allow possible chemical reactions to proceed to completion and not significantly affect reported sputter yields. A similar procedure was applied for the metal films grown on silicon substrates with a difference that only a single ion energy was used for a single sample of the metal film.

\subsubsection{X-Ray photoelectron spectroscopy}

After exposure, metal films exposed to nitrogen ions were transferred via ambient atmospheric environment to an X-Ray Photoelectron Spectrometer setup. To reduce ambient oxidation after ion exposure, the samples were maintained under vacuum after exposure, until the moment of transfer. Reference samples were measured immediately after deposition without additional sputter cleaning and were also transferred through atmosphere exposure. A maximum of 20 minutes of ambient pressure exposure is estimated for all samples reported here. The oxide formed on the samples due to this ambient exposure was $<1 \mathrm{~nm}$.

Samples were transferred into a Thermo-Fisher Theta probe Angle Resolved XRay photoelectron spectrometer which uses monochromatic Al- $K \alpha$ radiation (1486 $\mathrm{eV}$ ) with a spot size of $400 \mu \mathrm{m}$. Measurements were carried out at a pressure in the order of $1 \times 10^{-9}$ mbar as measured by an ion gauge. XPS spectra, except where explicitly mentioned, are reported for a take-off angle of $34.25^{\circ}$ with respect to the surface normal (which corresponds to the highest probing depth for the conditions of these experiments).

Nitrides of Group VI-VIII transition metals occur with nitrogen occupying interstitial sites [17]. Bonding in such phases involves a combination of metal-metal and metal-nonmetal interactions, leading to a variation in metallic and covalent bonding [17]. In the context of X-Ray photoelectron spectroscopy this implies that the structure of the metal core level spectra can at times be difficult to uniquely de-convolve into metal and metal nitride components [18]. The resulting lack of uniqueness of XPS spectral deconvolution was remedied in the following manner:

Core level spectra were obtained for the metal $3 \mathrm{~d}$ or $4 \mathrm{f}(\mathrm{Me})$ and nitrogen $1 \mathrm{~s}$ $(\mathrm{N} 1 \mathrm{~s})$ regions. In this way, $\mathrm{N} 1 \mathrm{~s} / \mathrm{Me}$ ratios involving total metal core intensities were obtained. This allowed comparisons of relative nitrogen content between target metals without spectral deconvolution. The experimentally obtained ratios were compared to ratios calculated from concentration profiles simulated by TRIDYN [19] as described in the following sections.

\subsection{Model and simulations}

Simulations of ballistic transport with dynamic composition changes were performed using TRIDYN (version 2017) [19]. The resulting depth profiles of implanted nitrogen are fed into a two-component multi-layer model for calculating N1s/Me XPS intensity ratios. 
We briefly discuss the TRIDYN code and parameters involved in the simulations as well as the XPS intensity calculations:

\subsubsection{Simulating sputtering and implantation depth with TRI- DYN}

TRIDYN tracks asymptotic trajectories of atomic collisions under the binary collision approximation. The collision interactions are modeled using a $\mathrm{Kr}-\mathrm{C}$ interaction potential [20] with the screening length approximated by the Firsov formulation [21].

The simulations were performed on a $80 \mathrm{~nm}$ thick target divided into 400 lamellae for a fluence of $2 f_{0} \sim 4 \times 10^{18}$ ions $/ \mathrm{cm}^{2}$ (where $f_{0}$ is the experimentally determined fluence), where the factor 2 comes from the assumption that all the incoming molecular nitrogen ions dissociate upon collision with the target surface. In order to account for the energy reorganization within the molecule, an incident molecular ion with energy $E_{0}$ is simulated as impact of two atomic ions, each with an energy of $E_{0} / 2$. For comparing experimental and simulated results, all text and graphs in this work always refer to the incident energy $E_{0}$. The beam was modeled as a combination of $\mathrm{N}^{+}$and $\mathrm{N}_{2}^{+}$, with the $\mathrm{N}^{+}$concentration set to $20 \%$ of the total beam content. $\mathrm{N}^{+}$ions retain their initial energy $E_{0}$. Increase in $\mathrm{N}^{+}$concentrations in the ion beam leads to a higher metal sputter yield below $100 \mathrm{eV}$ and shallower implantation depth. It also extends the sputter threshold to lower energies in comparison to a purely molecular ion beam. Setting the concentration to $20 \%$ gives us an upper limit to the increase in sputter yield due to $\mathrm{N}^{+}$impurities.

Reactions between ion and target are not simulated in the present code. However, atomic fractions from incorporation by ballistic particle deposition in the target can be recorded. This can be used either to suppress over-stoichiometry or limit the incorporation of projectiles through the parameter, ires [22]. It is handled in two major ways:

In the first case, we employ a method of local saturation. Here, each lamella can saturate discretely in nitrogen concentration $\left(N_{\text {conc }}\right)$ upto a pre-defined saturation fraction $N_{\text {sat }}$. Any nitrogen subsequently implanted in the lamella is transferred to the neighboring lamella where the concentration is less than $N_{\text {sat }}$. If the nearest lamella is the surface, the nitrogen is re-emitted out to vacuum. This mimics a model involving short range (lateral) diffusion and allows for out-diffusion of implanted nitrogen. When an incoming nitrogen adsorbs (implants) on a site (either on a surface or implanted in a lamella in depth), it does so on both metal and nitrogen sites unless the surface (lamella) is fully saturated [23]. We shall refer to this type of incorporation as Mode 1.

In the second case, the incoming nitrogen only adsorbs (implants) on a metal site (nitrogen sites are considered non-reactive for further nitrogen uptake). We shall refer to this as Mode 2. Mode 2 allows for a slow incorporation of ions in the target and can mimic either low concentrations of ion implantation or a target which forms clusters of stoichiometric compound in an otherwise metallic matrix. Here, metal sites become less available with increasing nitrogen incorporation, which leads to higher out diffusion of additional incoming nitrogen. The concentration profile asymptotically reaches $N_{\text {sat }}$ as a function of fluence. Both modes can be controlled 
by the choice of $N_{\text {sat }}$, which we fix to the atomic fraction of stoichiometric nitrides as an upper limit. Allowing nitrogen to be freely incorporated leads to the atomic fractions to tend quickly to an unrealistic 'pure nitrogen' surface.

The difference in the generated concentration depth profiles is shown in Figure 1a for Mo irradiated by $300 \mathrm{eV} \mathrm{N}_{2}^{+}$ions. The two modes show features of a saturation behavior controlled by $N_{\text {sat }}$ (Mode 1) and gradual uptake of nitrogen leading to an overall under-stoichiometric nitride (Mode 2). Applying Mode 1 and Mode 2, it is possible to generate collision cascades within a simulated target with a high or low reactivity, respectively. Chemical effects and diffusion can be sensitive to temperature which TRIDYN cannot simulate. The simulations thus agree best at low sample temperatures, as in our case, where external heating is not applied and radiative heating from the plasma increases sample temperature at most up to $50^{\circ} \mathrm{C}$.

TRIDYN requires a surface binding energy (SBE) matrix to evaluate sputtering events for cascades in a metal and metal nitride. The matrix elements are $S B E_{M e-M e}, S B E_{N-N}$ and $S B E_{M e-N}$, representing interactions between metal atoms, between nitrogen atoms and metal atom with nitrogen atoms, respectively. The SBE matrix element values are approximated as [19, 24] :

$$
\begin{gathered}
S B E_{M e-M e}=U_{S} \\
S B E_{M e-N}=\frac{1}{2} U_{S}+\frac{n+m}{2 n m} \Delta H_{f}+\frac{n+m}{4 n} \Delta H_{d i s s}^{N} \\
S B E_{N-N}=0.1 \mathrm{eV}
\end{gathered}
$$

where $U_{S}$ is the sublimation energy of the metal, $\Delta H_{f}$ is the formation energy of the nitride and $\Delta H_{\text {diss }}^{N}$ is the dissociation energy of molecular nitrogen gas $(9.8$ $\mathrm{eV}) . S B E_{N-N}$ represents the interaction of nitrogen atoms with other nitrogen atoms in the metal. It can usually be neglected [24].

Nitrogen in nitrides can recombine and diffuse to the surface, and the interaction can be of the order of the physisorption energy. $S B E_{N-N}$ is chosen to be in the order of adsorption energy of $N_{2}$ on metal surfaces to account for some interaction of gas species with the metal. Reported adsorption energies vary from $0.1 \mathrm{eV}$ for $\mathrm{W}$ [25], upto $0.4 \mathrm{eV}$ for $\mathrm{Pd}$ [26], and $0.8 \mathrm{eV}$ for $\mathrm{Mo}$ [27]. Ruthenium was reported to allow negligible adsorption of nitrogen [28]. Our simulations however did not show significant changes in the yield and implantation results upon varying $S B E_{N-N}$ from $0.8 \mathrm{eV}$ to $0.1 \mathrm{eV}$. Their low values do not influence the yields since $S B E_{M e-N}$ values are at least an order of magnitude larger. However, we include them here for completeness.

$n$ and $m$ are the stoichiometric coefficients of the formed metal nitride $M e_{n} N_{m}$ $\left(n=1, m=1\right.$ for $\mathrm{WN} ; n=2, m=1$ for $\left.\mathrm{Mo}_{2} \mathrm{~N}\right)$. Formation energies of nitrides of $\mathrm{Pd}$ are not readily available and we set them to $+1 \mathrm{eV}$ (endothermic). The exact value is of little consequence as we shall see in Section 4.4.1. The parameters and values used for the simulations are summarized in Table 4.1. In the case of Mode 2 , where fractional incorporation of nitrogen occurs, we assume $S B E_{M e-N}$ is not significantly affected by compound formation and set $S B E_{M e-N}=S B E_{M e-M e}=U_{S}$. 
Table 4.1: Parameters used for TRIDYN simulations

\begin{tabular}{lllllll}
\hline \multicolumn{7}{c}{ Parameters } \\
\hline \hline Element & $\begin{array}{l}\text { Enthalpy of } \\
\text { Formation: } \\
\text { Nitride }(\mathrm{eV})\end{array}$ & $\begin{array}{l}\text { Nitride } \\
\text { density } \\
\left(\mathrm{g} / \mathrm{cm}^{3}\right)\end{array}$ & $\begin{array}{l}\text { Saturation } \\
\text { Atomic } \\
\begin{array}{l}\text { Fraction } \\
\left(\mathrm{N}_{\text {sat }}\right)\end{array}\end{array}$ & $\begin{array}{l}\text { SBE } \\
(\mathrm{Me}-\mathrm{Me})\end{array}$ & $\begin{array}{l}\text { SBE } \\
(\mathrm{Me})\end{array}$ & $\begin{array}{l}\mathrm{N}_{2}^{+}: N^{+} \\
(\mathrm{eV})\end{array}$ \\
\hline $\mathrm{Mo}$ & -0.72 & 9.4 & 0.33 & 6.82 & 7.63 & $80: 20$ \\
$\mathrm{Ru}$ & +0.925 & $9.48^{a}$ & 0.5 & 6.74 & 9.20 & $80: 20$ \\
$\mathrm{Pd}$ & - & $11^{b}$ & 0.1 & 3.89 & 9.52 & $80: 20$ \\
$\mathrm{~W}$ & -0.82 & 16 & 0.5 & 8.90 & 10.1 & $80: 20$ \\
\hline
\end{tabular}

$\overline{{ }^{a} \text { Reference:[29] }}$

${ }^{b}$ Reference:[30]

\subsubsection{Reconstructing XPS intensities}

In order to compare results of TRIDYN depth profiles to experimentally observed nitrogen content, a reconstruction of the XPS N1s/Me intensity ratios is necessary. XPS intensity ratio reconstruction is possible for arbitrary concentration profiles. A multi-lamellar model was successfully demonstrated by Meisl et al. [31] for estimating the validity of simulated depth profiles of nitrogen in tungsten generated by the Monte Carlo code SD.TRIM.SP [32] by comparing it to sputter depth profiling by XPS. We apply a similar theoretical procedure to reconstruct energy dependent intensity ratios for $\mathrm{Mo}, \mathrm{Ru}, \mathrm{Pd}$ and $\mathrm{W}$.

In general, the intensity of a photoelectron emission line from a thin film of material $\mathrm{X}$ can be written as [31, 33]:

$$
I_{X}=\sigma_{X} \beta_{X} \Phi_{p h} \int_{0}^{\infty} \rho(z) \exp \left(-\int_{0}^{z} \frac{1}{\lambda(z) \cos (\alpha)} d z^{\prime}\right) d z
$$

where $\sigma_{X}$ is the element's subshell photoelectric cross-section [34], $\beta_{X}$ is the asymmetry parameter [35] and $\phi_{p h}$ is the X-Ray photon flux. $\rho$ is the atomic density, $\lambda$ is the inelastic mean free path (IMFP) [36] and $\alpha$ is the angle of detector with respect to the surface normal. $\Phi_{p h}$ vanishes upon comparison of intensity ratios assuming a stable X-Ray source operation during spectrum acquisition. Considering a thin film to be composed of $\mathrm{k}$ discrete lamellae, equation 4.4 can be expressed as:

$$
\begin{aligned}
& I_{X}=\sigma_{X} \beta_{X} \Phi_{p h} \sum_{k=1}^{N} \rho_{k}\left(1-\exp \left(-\frac{\Delta z}{\lambda_{k} \cos (\alpha)}\right)\right) \exp \left(-\sum_{j}^{k-1} \frac{\Delta z_{j}}{\lambda_{j} \cos (\alpha)}\right) ; \\
& j=1,2, \ldots \forall k \geq 2
\end{aligned}
$$

A graphical representation of the model setup leading to equation 4.5 is shown in Figure 4.1b. The term in the first parenthesis describes the photoelectron intensity 
from a lamella $k$. The outgoing intensity will be attenuated on its path to the detector while passing through $k-1$ layers and is factored in through the latter exponential function. Equation 4.5 cumulatively sums the intensities originating from each lamella within the sample and knowing the incident photon flux, predicts the XPS intensity of an atomic specie in the thin film.
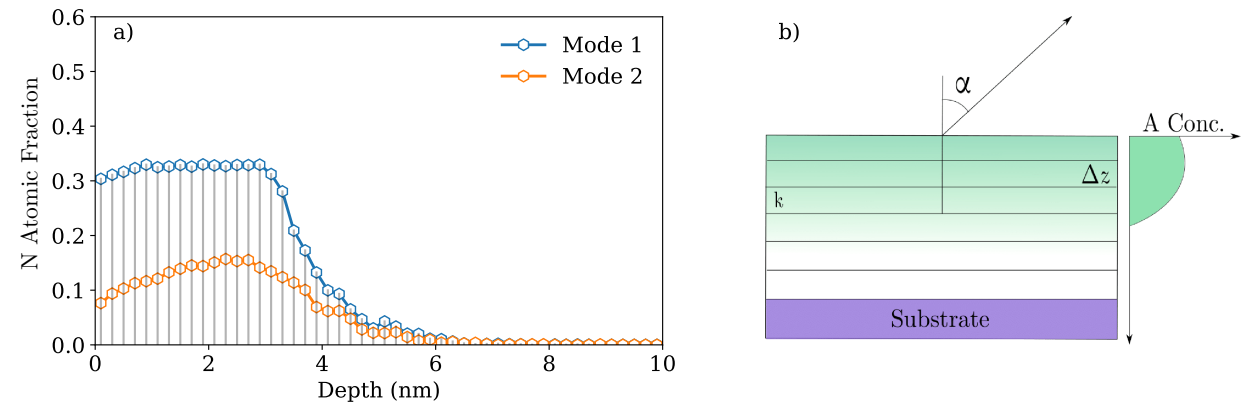

Figure 4.1: a)TRIDYN simulations of $300 \mathrm{eV}$ nitrogen on Mo illustrating differences in Mode 1 and Mode 2 of incorporation and build-up of nitrogen within the target; gray lines depict lamellar discretization of target b) schematic for setup of equation 4.5: Depth profiles from simulations are discretized into lamellae each contributing a certain fraction to the total intensity of the element from the film. A cartoon of the profile of element A is shown on the right of (b).

For comparison with experimental data, we report our measured intensity as normalized intensity (area) according to:

$$
I_{\text {normed }}=\frac{I_{\text {expt }}}{T(E) E C F}
$$

where $I_{\text {expt }}$ is the experimentally obtained intensity, $\mathrm{T}(\mathrm{E})$ is the detector transmission function dependent on photoelectron kinetic energy and ECF is the energy correction factor, set to $E^{0.6}$ for Scofield sensitivity factors [34] and $E$ is the photoelectron energy.

\subsection{Results}

We begin by evaluating experimental results in the following manner: First, the measured sputter yields are compared to TRIDYN simulations. It is observed that a chemical reactions can influence the magnitude of the yield. Next, the nature of the chemical interactions are studied through XPS spectra and evidence of nitride formation on 'inert' metals is discussed. The TRIDYN simulations of ballistic implantation are used to explain the nitrogen content in each metal film.

\subsubsection{Sputter yields}

Thick films grown on the QCM allowed for measurements of sputter yields of the metals. Using SBEs obtained from thermodynamic considerations - as opposed to its use as a fitting parameter - is insightful in assessing nitrogen sputtering and 
retention. It facilitates a more physically constrained comparison between elemental and compound sputtering while separating effects due to modification of SBE and altered collision cascades caused by $\mathrm{N}$ retention. It is expected for metals with known stable nitrides (W, Mo) to behave according to Mode 1 while relatively inert materials would behave according to Mode 2 .

a) $\mathrm{Pd}$

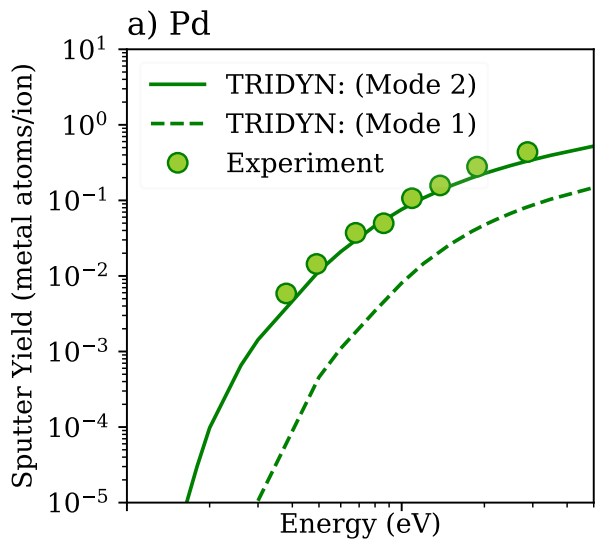

c) Mo

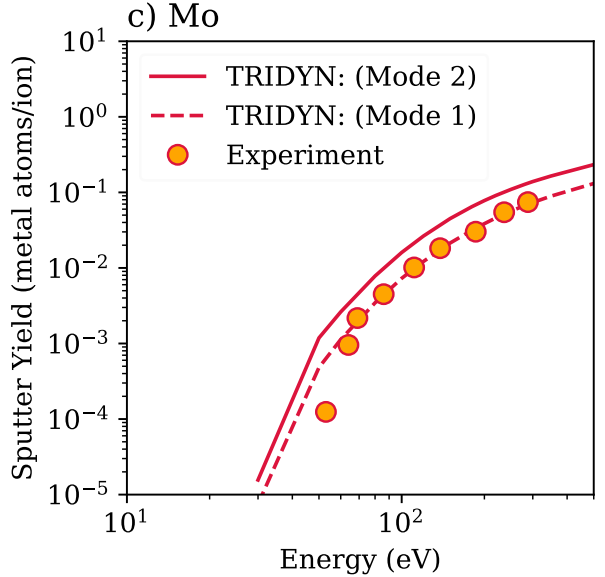

b) $\mathrm{Ru}$

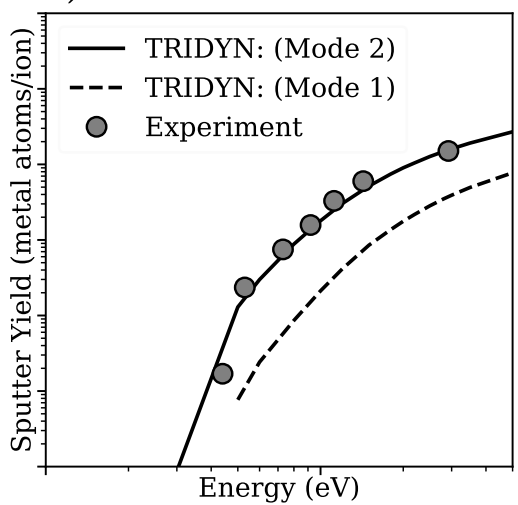

d) $\mathrm{W}$

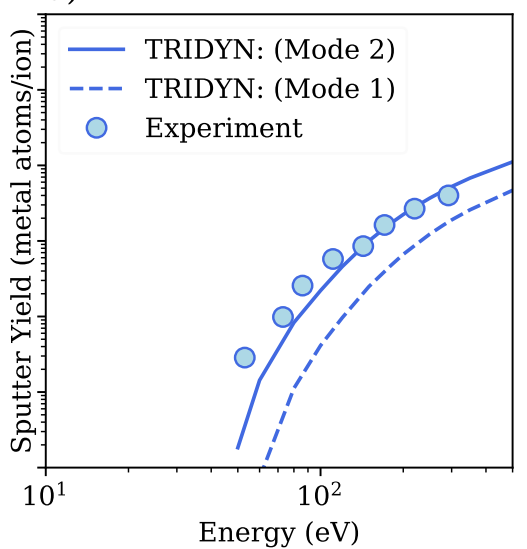

Figure 4.2: Experimental sputter yield results (dots) from the QCM and simulated TRIDYN predictions for stoichiometric (dashed lines) and under-stoichiometric (solid) implantation are shown. a) Palladium; b) Ruthenium exhibit a yield due to sub-stoichiometric implanted nitrogen; c) Mo exhibits a yield dominated by stoichiometric implantation; and d) Tungsten deviates from stoichiometric compound induced sputter yield possibly due to high spatial frequency roughness.

Figure 4.2 shows the experimental and simulated yields for all four elements. As experimental yields were measured at the steady state of incorporated and sputtered nitrogen, the mass loss is considered mainly due to metal removal. It is clear from the simulations shown in Figure 4.2 that the formation of a compound, which is simulated according to Mode 1 (dashed lines in Fig 4.2), results in a lowering of the sputter yield, compared to simulations using Mode 2, which only accounts for 
implantation. This is a direct result of the $S B E_{M e-N}$ being larger than $S B E_{M e-M e}$ of the pure metal (refer to Table 4.1). Pd does not readily form stable nitrides at low-ambient pressures and the experiments agree with simulations of pure palladium sputtering with implantation of nitrogen (Mode 2) to an atomic fraction of 0.1 . Ru forms a stable nitride [29, 37], despite a positive enthalpy of formation. However, the sputtering is dominated by $\mathrm{N}$ retention causing cascade modifications (Mode 2 ) and a direct effect of a change to the surface binding energy is not observed. Mo readily forms nitride compounds, and this is evident in the simulated sputter yield which follows simulations accounting for both effects of cascade modification due to nitrogen retention and an increased $S B E_{M e-N}$ (Mode 1). Tungsten is also known to form nitrides, however, the behavior of sputter yields is unlike that of Mo. This could potentially be due to a higher roughness of the tungsten film (See section 4.5.1). Summarizing, we found that the binary collision approximation is capable of predicting sputter yields down to near-threshold regions for reactive ion bombardment. Yields for metals which do not readily form nitrides ( $\mathrm{Ru}$ and $\mathrm{Pd}$ ) can be explained by accounting for implantation only without modifying the compound surface binding energy (Mode 2). Yields for metals which readily form nitrides can be explained by a combination of implantation and binding energy change (Mode 1). Tungsten shows a higher sputter yield than predicted by Mode 1 due to dominant roughness variations. Investigation of compound formation for metals to better understand validity of the modes follows.

\subsubsection{Nitrogen incorporation}

To investigate compound formation, films grown on silicon wafers were exposed to fluences equal to those for the QCM experiments. The high fluence allowed for a steady state in sputtering and incorporation and the XPS spectra from the metal components for samples irradiated with selected ion energies are shown in Figure 4.3. Mo and $\mathrm{W}$ show marked differences in core level spectra before and after nitrogen ion bombardment. The Mo3d region in Figure 4.3a shows peaks at $229.1 \mathrm{eV}$ and $232.4 \mathrm{eV}$, consistent with reported binding energies of an oversaturated $\mathrm{Mo}_{2} \mathrm{~N}$ [38]. The W4f region exhibits nitride peaks at $32.6 \mathrm{eV}$ and $34.8 \mathrm{eV}$ corresponding to the spin-orbit states of $\mathrm{W} 4 \mathrm{f}_{7 / 2}$ and $\mathrm{W} 4 \mathrm{f}_{5 / 2}$ respectively, comparable with reactively deposited WN sputtered by $\mathrm{Ar}^{+}$ions [39]. Both metals show traces of oxide due to exposure to ambient conditions. Ru and Pd XPS spectra demand closer inspection. The $\mathrm{Ru}_{3} \mathrm{~d}_{5 / 2}$ peaks in Figure $4.3 \mathrm{c}$ do not show any observable shift in binding energy within the instrument resolution. The oxide content within the sample due to the sample transfer is not uniquely quantifiable and possibly overlaps with any nitride present. Contamination from carbon on ruthenium adds to errors in quantification due to overlap of the $\mathrm{C} 1 \mathrm{~s}$ peak with the $\mathrm{Ru} 3 \mathrm{~d}_{3 / 2}$ peak. Carbonaceous impurities were estimated to be similar between exposed samples by comparing the Ru3d $5 / 2$ and $\mathrm{Ru}_{3} \mathrm{~d}_{3 / 2}$ peak ratios. A nitride component is assigned for deconvolution of core lines accounting for broadening of the metal spectrum. Exact binding energies for a thin nitride/nitrogen implanted layer could not be determined. Core level spectra of $\mathrm{Pd}$ show a marked broadening of $3 \mathrm{~d}$ peaks with a change in asymmetry. Broadening of core spectra is indicative of either changes in the local order (amorphization) 

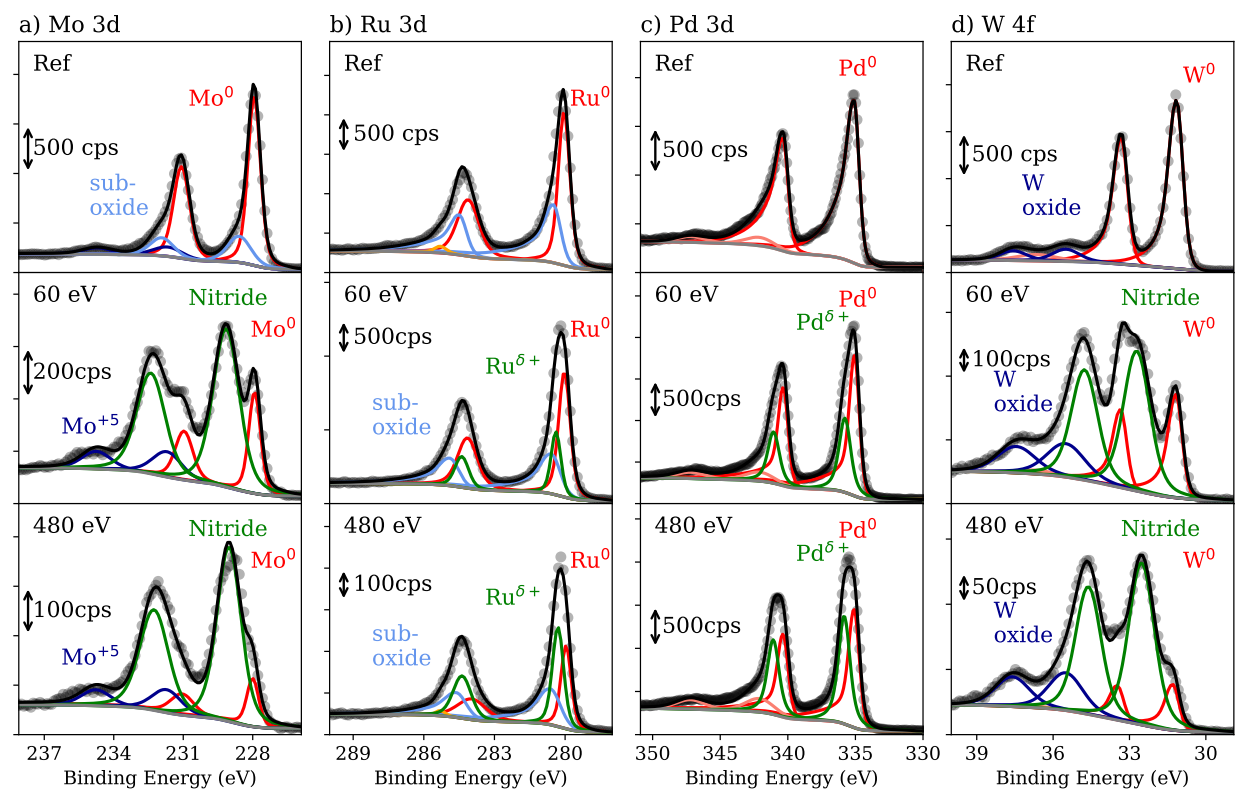

Figure 4.3: Photoelectron emission spectra for a) Molybdenum 3d, b) Ruthenium 3d, and c) Palladium 3d and d) Tungsten 4f, core levels, for selected ion energies. The spectra show oxide (substoichiometric and stoichiometric) peaks due to atmospheric exposure (light and dark blue). Points indicate measured data, solid lines fitted components.

within the crystal or electron screening variations due to bonding. Amorphization due to ballistic impact was ruled out by etching the reference surface with $500 \mathrm{eV}$ $\mathrm{Ar}^{+}$and measuring the broadening of the peak at the end of the etch step which was found to be negligible. Broadening could also be attributed to the presence of oxygen attached to surface atoms and its resolution is difficult due to the overlap of the Pd3p orbital with O1s. However, the absence of an oxygen KLL Auger line intensity in the survey spectra rules out oxide formation during the sample transfer. Observing peak intensity changes at various take-off angles, a binding energy of $335.9 \mathrm{eV}$ is assigned to a nitrogen implanted palladium. This is comparable to the value of $335.5 \mathrm{eV}$ assigned to a palladium with a fractional positive charge $\left(\mathrm{Pd}^{\delta+}\right)$ after interaction with $\mathrm{N}$ atoms [40]. In order to confirm any nitridation, we analyze the positions of any N1s peaks present.

The N1s spectra illustrate the extent of nitrogen incorporation within the analyzed sample volume. Metal-nitrogen bonds typically occur at binding energies of $\sim 397-398 \mathrm{eV}$ in the N1s region [41], clearly evidencing nitride formation. Mo and $\mathrm{W}$ show clear peaks at energies of $397.2 \pm 0.1$ and $397.1 \pm 0.2 \mathrm{eV}$ respectively, which are attributed to a metal nitride. Ruthenium is interesting as the N1s line developed upon irradiation with $\mathrm{N}_{2}^{+}$ions represents the nitrogen content within the film, even though the metal peak shows no discernible shift. A peak with a binding energy at $397.7 \pm 0.2 \mathrm{eV}$ is assigned for nitrogen implanted in ruthenium. Pd shows a broad nitrogen peak in the reference measurement at $\sim 399-400 \mathrm{eV}$ which is re- 

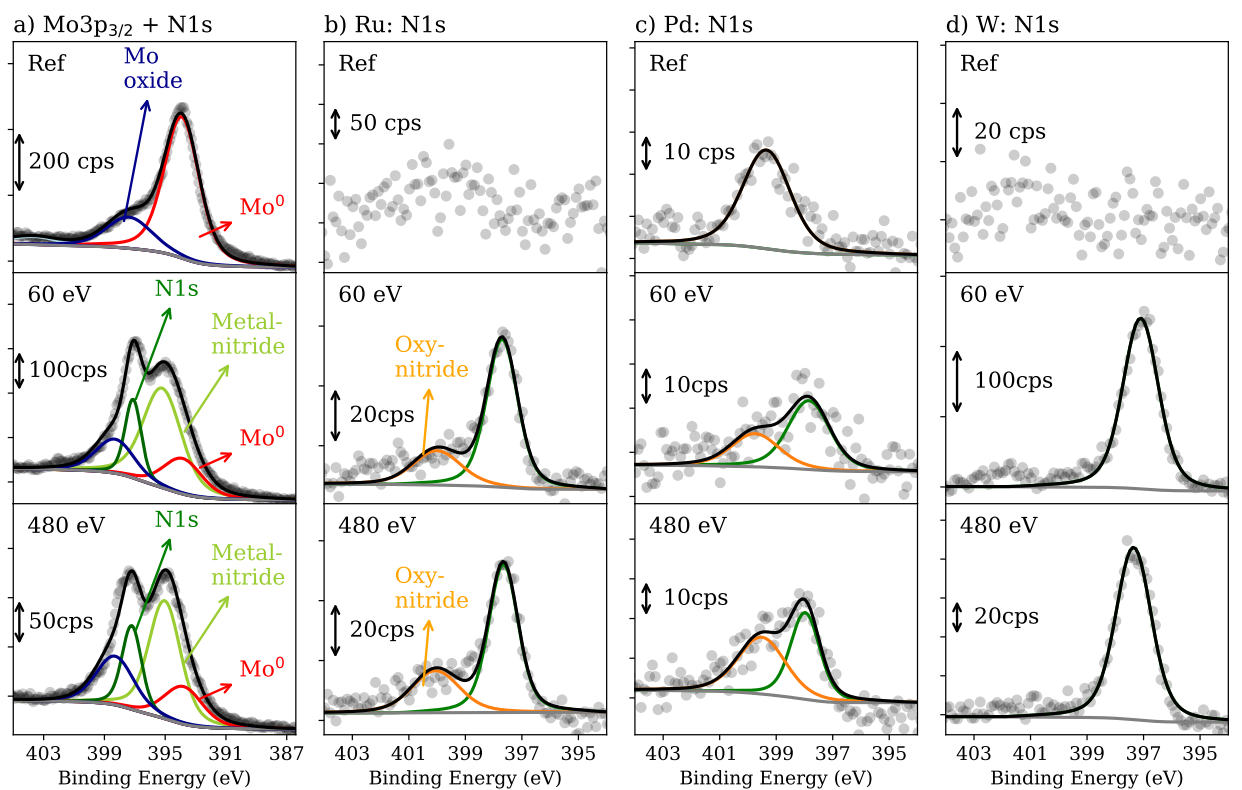

Figure 4.4: Photoelectron emission spectra of the N1s region for a) Molybdenum showing overlap of the N1s peak with the Mo $3 \mathrm{p}_{3 / 2}$ peak, and d) Tungsten: for take-off angles of $34.25^{\circ}$; and angle averaged peaks showing the nitride and oxy-nitride, nitride and adsorbed $\mathrm{N}$ peaks respectively for b) Ruthenium and c) Palladium:. Gray dots are measured data, solid lines are de-convolution fits to the data.

lated to adsorbed nitrogen atoms and nitrogen containing species (such as NO) on the surface [34]. The structure of this broad Gaussian changes upon nitrogen ion bombardment with the adsorbed nitrogen peak separating out into neutral $\mathrm{N}$ at 399 $\mathrm{eV}$ and implanted nitrogen possibly forming a nitride at $398 \mathrm{eV}$. The contribution of oxynitride species to the signal at higher binding energy $(399 \mathrm{eV})$ can be discounted due to the lack of a clear oxygen signal in the O KLL Auger region of the spectrum for $\mathrm{N}_{2}^{+}$exposed samples.

The N1s peaks indicate formation of nitride, but stoichiometry cannot be determined in all cases due to complexity of deconvolution. Mo and W metal peaks are well separated from the nitride peaks while $\mathrm{Ru}$ and $\mathrm{Pd}$ metal spectra are more complicated. Intensity ratios of measured nitrogen to the total metal however, do not suffer from problems of de-convolution and uniqueness of fits. Contamination by oxygen due to atmospheric exposure will lower intensity ratios and we expect this to not exceed a factor 2. We thus rely on the intensity ratios and TRIDYN simulations for understanding nitrogen transport and retention.

In order to compare experimental intensity ratios, we first look at depth profiles generated by TRIDYN which were used to model intensity ratios according to equation 4.5. TRIDYN simulations were performed according to the procedure in Section 4.3.1. Figure 4.5 shows the depth profiles of the implanted nitrogen for each target element for selected modes at the end of fluence. Within Mode 1 (Figure 


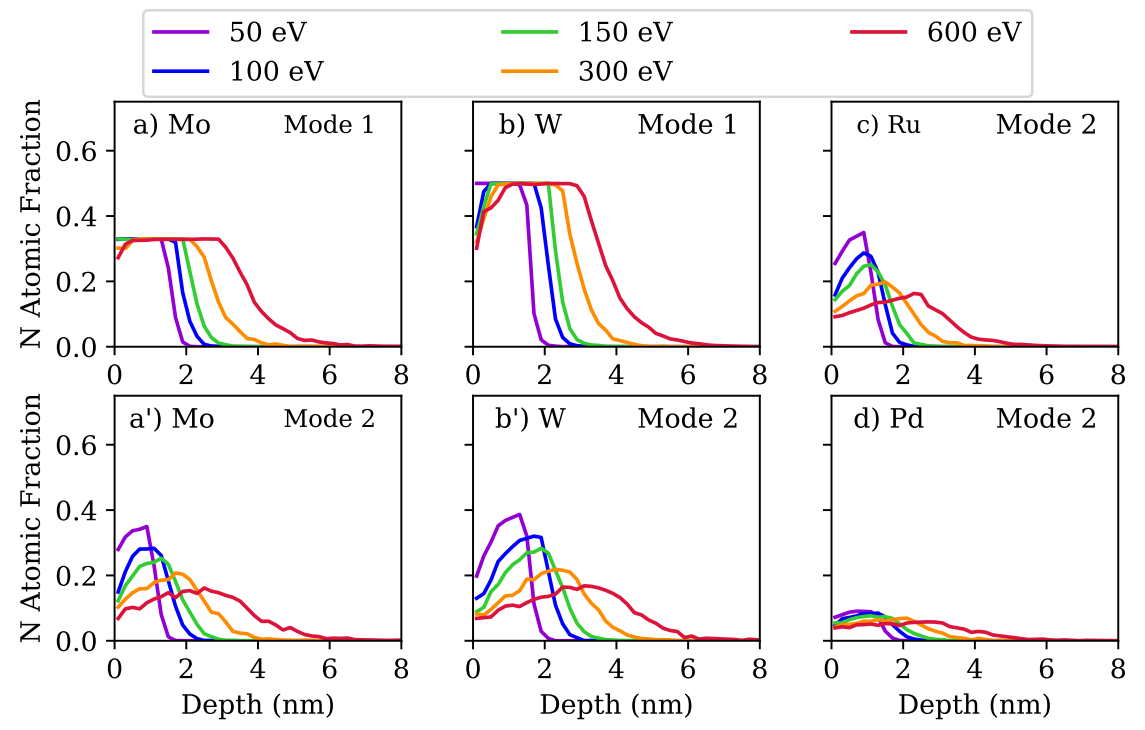

Figure 4.5: Nitrogen atomic fractions as estimated by TRIDYN for a, a') Mo; b, b') W; c) Ru; and d) Pd. Mode 1 describes a process of lateral local diffusion with nitrogen incorporated in both metal and nitride sites until $N_{\text {sat }}$. Mode 2 describes implantation of nitrogen into the target on metal sites only leading to an overall substoichiometric levels of nitrogen.

4.5a -b), the nitrogen content reaches stoichiometric values. The depth of implanted nitrogen increases with increasing ion energy as expected from the energy dependence of the range of incident ions. Furthermore, increasing ion energy creates a nitrogen deficient nitride surface due to preferential sputtering of surface nitrogen by incident ions. Simulations with Mode 2 (Figure $4.5 \mathrm{c}-\mathrm{d}$ ) for $\mathrm{Ru}$ and $\mathrm{Pd}$ show similar behavior in terms of nitrogen implantation depth increasing with ion energy with the notable difference that the implantation profile never reaches stoichiometry within the probed fluence.

Integration of the TRIDYN depth profiles to obtain $\mathrm{N} / \mathrm{Me}$ ratios was performed by applying equation 4.5 to the depth profiles as in [31]. This involved integrating the entirety of the simulated depth $(80 \mathrm{~nm})$ and setting the metal concentration to $\left(1-N_{\text {conc }}\right)$. The depth range contributing to the simulated signal is much smaller though, as accounted for through the IMFP of the outgoing photoelectrons (equation 4.4). A comparison of the theoretical N/Me ratios to experimental XPS intensity ratios for the target materials is shown in Figure 4.6. The experimental datasets exhibit two distinct features: First, the N/Me ratio remains relatively constant within $20 \%$ as a function of ion energy. Second, the intensity ratios vary by an order of magnitude upon changing target material. Intensity reconstruction from TRIDYN profiles shows differences in the nitrogen incorporation for different modes. Reconstruction depends on $\mathrm{N}$ concentration in the following manner as suggested by equation 4.5: Surface $\mathrm{N}$ contributes the most to the N1s intensity; Subsurface N contribution decays exponentially with depth, but having a higher N 
content in a particular lamella adds to the intensity contribution. Mode 2 shows a much lower intensity ratio of $\mathrm{N} / \mathrm{Me}$ due to fractional incorporation of $\mathrm{N}$. Pd and $\mathrm{Ru}$ data shows a better agreement with Mode 2, similar to the sputter yields in Figure 4.2. Reconstruction with Mode 2 suggests that the $\mathrm{N} / \mathrm{Me}$ ratio decreases with incident ion energy. This is because, as the energy is increased, $\mathrm{N}$ is transported deeper (lowering the $\mathrm{N}$ intensity contribution) and surface nitrogen is removed by sputtering (lowering $\mathrm{N}$ intensity). Similarly, decreasing the incident energy leads to accumulation of $\mathrm{N}$ closer to the surface (increasing $\mathrm{N}$ intensity). Mode 1 shows a higher $\mathrm{N} / \mathrm{Me}$ intensity ratio in all cases due to a higher (saturated) $\mathrm{N}$ content in the measurable depth. The energy dependence here is markedly different from Mode 2: Increasing energy causes only a small increase in $\mathrm{N} / \mathrm{Me}$ ratio because a saturation of $\mathrm{N}$ is present for all energies and transport of nitrogen deeper (increase in $\mathrm{N}$ intensity) is compensated by a deficiency in surface $\mathrm{N}$ content (decrease in $\mathrm{N}$ intensity). The behavior of Mo and $\mathrm{W}$ match well with Mode 1 incorporation indicative of a higher reactivity of these metals with $\mathrm{N}$, which is also consistent with the observation of clearly separated XPS peaks of metal nitride in the Mo $3 \mathrm{~d}$ and $\mathrm{W}$ 4f core levels. As pointed out before, the sputter yields for $\mathrm{W}$ observed experimentally are an outlier in the sense that they match Mode 2 simulations better than predictions by Mode 1, possibly due to surface roughness which we shall discuss in the following section.

\subsection{Discussion}

\subsubsection{Sputtering and compound formation}

TRIDYN simulations are in good agreement for the sputtering and possible implantation for three of the four metals studied. Mo and $\mathrm{Ru}$ data of yields and $\mathrm{N} / \mathrm{Me}$ XPS intensity ratios are well corroborated by the simulations under the assumptions applied for the binding energy, implantation mode and atomic ion impurities. Molybdenum exhibits a stoichiometric nitride, $\mathrm{Mo}_{2} \mathrm{~N}$, formed upon ion bombardment as is evidenced from the N1s peak position. This is well described within Mode 1 where transport of nitrogen within the Mo film is largely due to ballistic collisions and subsequent stopping and reaction. Ruthenium shows a lower $\mathrm{N} / \mathrm{Me}$ intensity ratio, which is explained by its relative chemical inertness and a higher amount of reflected nitrogen. The transport of nitrogen in this case is also explained by ballistic collisions. The presence of the nitride N1s signal indicates the implanted nitrogen reacts with the $\mathrm{Ru}$ upon stopping. This amount of implanted nitrogen however, does not contribute to changing the overall surface binding energy of the target, rather, the modifications of the collision cascades due to different energy transferred by back-scattered or recoiled atoms upon collision of an incident ion with a target $\mathrm{Ru}$ or implanted $\mathrm{N}$ atom is responsible for the observed sputter yields.

Sputtering of $\mathrm{Pd}$ by $\mathrm{N}_{2}^{+} / \mathrm{N}^{+}$showed results which were successfully reconstructed in simulations (using Mode 2). Simulations at various $\mathrm{N}$ implantation fractions were carried out and an atomic fraction of $N_{\text {sat }} \sim 10 \%$ showed the best results. The XPS peaks of N1s point towards the development of a possible nitride phase due to im- 

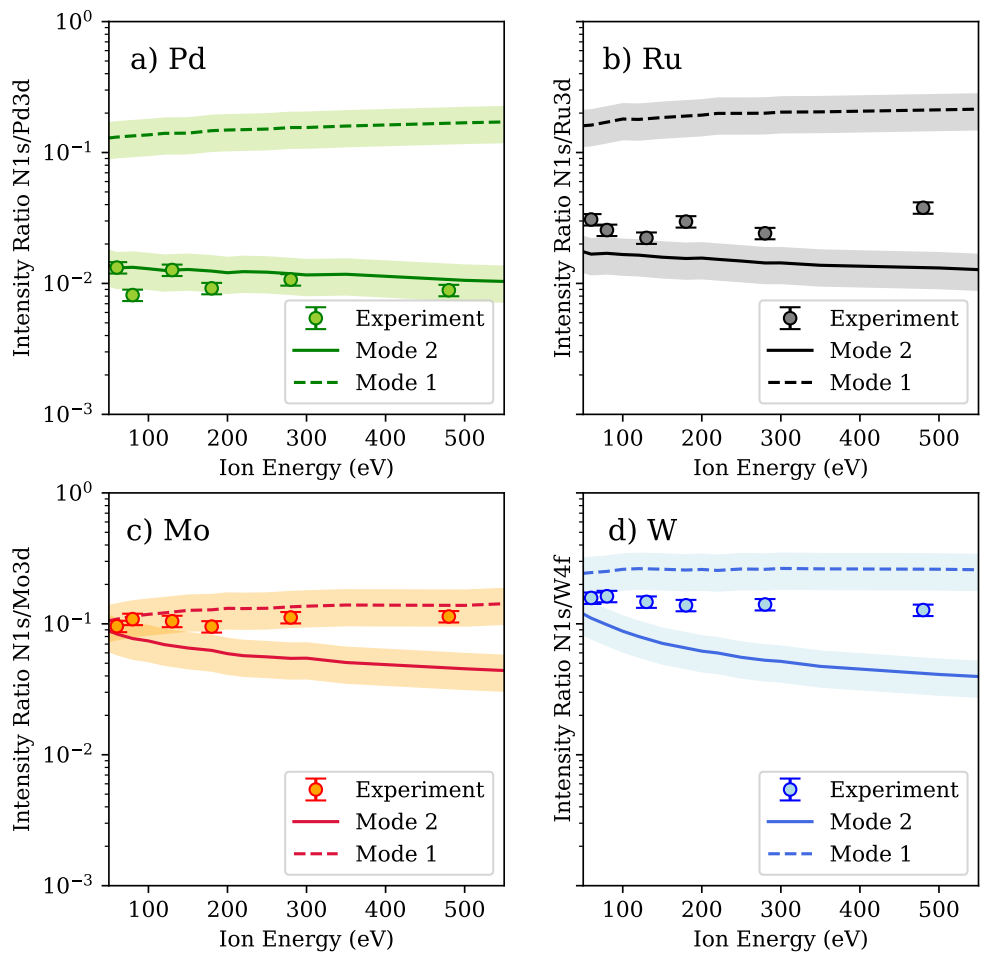

Figure 4.6: Intensity ratios of nitrogen 1s to entirety of Metal 3d/4f spectrum from XPS measurements for a) Pd; b) $\mathrm{Ru}$; c) Mo and d) W. The lines are calculated intensity ratios for the ion-target combinations from the depth profile resulting from TRIDYN simulations for a nitrogen incorporation upto nitride stoichiometry (mode 1: dashed) and metal sputtering with nitrogen implantation (mode 2: solid). Filled areas depict error margins of the calculations. 
planted nitrogen. Given the limits imposed by the energy resolution of a laboratory XPS system, the metallic Pd3d peaks cannot be clearly separated from the nitride contributions. Ru also suffers from this limitation rendering a stoichiometry evaluation difficult. However, an N1s peak in the region of a metal nitride, is indicative of the presence of nitrogen-metal bonds.

Experimental results of $\mathrm{W}$ sputter yields under nitrogen bombardment deviate under the assumptions of compound formation and SBE changes discussed in Section 4.3.1. Tungsten exhibits sputter yields that are in line with the Mode 2 approximation while experimental XPS N/Me ratios are best described as a nitrogen implantation up to a stoichiometric nitride saturation as per Mode 1, which is also in line the negative enthalpy of formation of tungsten nitride. The deviation of the experimental sputter yields compared to the theoretical predictions according to Mode 1 may be caused by surface roughness of the target. As TRIDYN assumes a flat surface approximation, the effect of roughness may play a significant role in the experiments for $\mathrm{W}$, which cannot be simulated in the present version of the code. The roughness dependence of the sputter yield is known to be a complex function of the implantation depth and the surface correlation width [42], and enhancements can be expected to be up to a factor 2.5. W grown on silicon substrate samples had an RMS roughness $\left(R_{q}\right)$ of $1 \mathrm{~nm}$ as determined from a $1 \mu \mathrm{m} \times 1 \mu \mathrm{m}$ AFM scans [see Supplementary Information]. This was much larger than that of other elements $\left(R_{q} \sim 0.3-0.5 \mathrm{~nm}\right)$ studied. At such lateral scanning ranges, roughness features of the order of a few $\mathrm{nm}$ are dominant contributors to $R_{q}$. While long range roughness from metal grown on a QCM has been shown to not significantly alter sputter yields [10], local roughness variations would influence the incidence angles of the ions and consequently sputter yields. This has been evidenced in the literature for W sputtering by carbon (C) ions where TRIDYN simulations correctly predicted fluence dependent incorporation of implanted $\mathrm{C}$ while deviation in sputter estimates occurred due to roughness [43].

\subsubsection{Nitrogen reaction probability}

All metals studied exhibit an N1s peak in the XPS spectra whose position represents a metal nitride. The susceptibility of a metal to form a nitride is determined by the reaction cross-section. Using the available data, an estimate of this cross-section is made. The interaction of a nitrogen beam with a metal surface can be broken down into the following elementary reactions :

$$
\begin{gathered}
N_{2}^{+}+e_{\text {metal }}^{-} \longrightarrow N_{2} \\
N_{2} \longrightarrow 2 N \\
n M e+m N \longrightarrow M e_{n} N_{m} \\
M e_{n} N_{m}+N \longrightarrow M e_{n-1} N_{m}+M e+N
\end{gathered}
$$




$$
M e_{n} N_{m}+N \longrightarrow M e_{n} N_{m-1}+2 N
$$

The incoming nitrogen molecular ion is neutralized by metal surface electrons via a resonant or auger electron process (equation 4.7). Collision with the metal surface leads to dissociation of the energetic neutral nitrogen molecule forming atomic nitrogen (equation 4.8). Further, once they lose enough energy the nitrogen atoms react with the metal and form a nitride (equation 4.9). In addition to retention, the nitrogen in the metal lattice is also sputtered along with metal atoms due to nitrogen ion bombardment (equation 4.11) .

Ion neutralization occurs with a $99 \%$ probability in a region of $0.5-0.6 \AA$ from the metal surface $[44,45]$. Crudely approximating the ion beam to consist of molecular nitrogen ions in a single electronic excited state $\left({ }^{1} \Sigma_{g}^{+}\right)$and no rotational or vibrational excitations, the threshold energy for dissociation of $\mathrm{N}_{2}$ is $9.76 \mathrm{eV}$. Bombarding energies in the present study are at least a factor 5 higher than this threshold and nearly every incident $\mathrm{N}_{2}$ can be considered to be dissociated upon collision and available for reaction. Rotational and vibrational states may lower this threshold of dissociation, which enforces the assumption [46]. Further, assuming neutralization and collision induced dissociation to be constant over the energy range, reaction and sputtering of nitrogen are then competing processes involved in the retention of nitrogen in the target. The amount of nitride product $\mathrm{P}_{N}$ formed is approximated by [47]:

$$
P_{N}=M_{0}\left(\frac{\sigma_{R}}{n \sigma_{R}+m \sigma_{S}}\right)\left(1-\exp \left(-\frac{n \sigma_{R}+m \sigma_{S}}{A} R_{0}\right)\right)
$$

where $M_{0}$ is the amount of metal, $\sigma_{R}$ is the reaction cross-section and $\sigma_{S}$ is the sputtering cross-section. $\sigma_{S}$ is directly proportional to the sputter yield $(Y)$ when the beam interacts with a certain areal density of atoms $n_{0}$, within the projected range. In principle, both cross-sections vary as a function of the ion kinetic energy which evolves as the collision cascade progresses, and the dependence is not easily known. We shall limit the discussion to the dependence of the ratios of the cross-sections as a function of incident energy only. $\mathrm{n}$ and $\mathrm{m}$ are stoichiometric coefficients for the metal nitride $\left(\mathrm{Me}_{n} \mathrm{~N}_{m}\right)$ and $R_{0}$ is the number of ions incident on the surface with an area $A$. In the limit of saturation $R_{0} \rightarrow \infty$ as the case of current experiments, the product to metal ratio is expressed as:

$$
\frac{P_{N}}{M_{0}}=\frac{1}{n+m\left(\sigma_{S} / \sigma_{R}\right)}
$$

The term on the left hand side of equation 4.13 describes the XPS intensity ratios. The right hand side determines the retention of nitrogen through the ratio of sputtering to reaction cross-section. Upon rearranging the terms it is clear that above the sputter threshold, $\sigma_{S}$ is proportional to $\sigma_{R}$. Determining the absolute value of $\sigma_{R}$ experimentally would require fluence dependent measurements below saturation fluence. However, knowing the intensity ratios and sputter yields, equation 4.13 can be evaluated to estimate $\sigma_{R} \cdot \sigma_{R}$ here represents an "apparent" reaction cross-section involving a combination of pathways for reaction as well as 

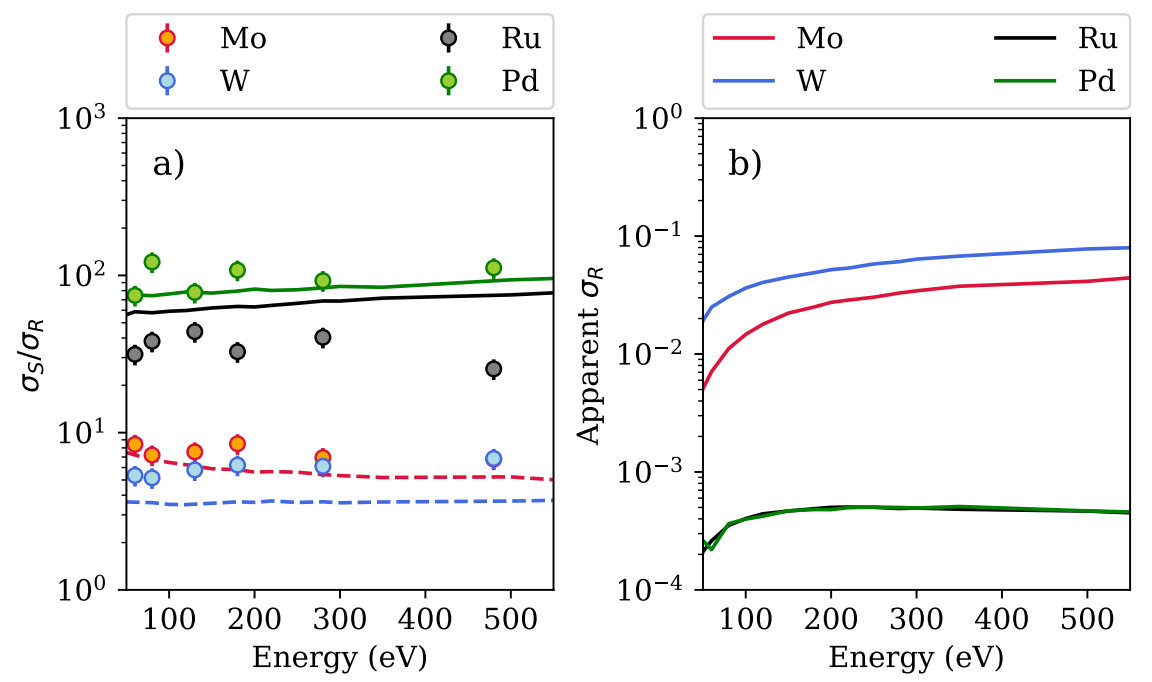

Figure 4.7: a) Experimental 'apparent' cross-section ratios as a function of energy derived from equation 4.13. Retention channels include implantation and reaction while sputtering channels where nitrogen loss occurs include sputtering and surface oxide formation (in experiments). TRIDYN predictions of sub-stoichiometric (solid lines) and stoichiometric (dashed lines) implantation are also depicted. b) Apparent reaction cross-section from equation 4.13 in the limit of saturation and setting $\sigma_{S}=$ Simulated $\mathrm{N}$ partial sputter yield.

implantation. Figure 4.6 shows that the intensity ratio for each element varies fractionally with increase in energy. As the sputter yield (and $\sigma_{S}$ ) increases, $\sigma_{R}$ would have to increase as well in order to maintain the same XPS intensity ratio.

Relative ratios of sputtering and retention are extracted from experiments based on equation 4.13 and shown in Figure 4.7a as function of incident ion energy. Over the energy range probed, the ratio of the cross-sections remains nearly constant. We know from the QCM experiments that the sputtering cross-section (proportional to sputter yield) of the metal increases non-linearly with increasing energy. Experimental measurements of $\mathrm{N}$ sputtering were not available in the current scheme of the setup. TRIDYN supplies as an output partial yields of the metal and N. The reasonable agreement of the experimental and predicted metal sputter yields gives confidence in the cascades and partial yields simulated by TRIDYN. Setting $\sigma_{S}$ to be equal to the partial yield of $\mathrm{N}$, the behavior of $\sigma_{R}$ over the energy range can be understood. Figure $4.7 \mathrm{~b}$ shows trends in $\sigma_{R}$ over the energy range probed. $\mathrm{Pd}$ and $\mathrm{Ru}$ show a similar range of $\sigma_{R}$ in comparison Mo and $\mathrm{W}$ whose reaction crosssections are about $10^{2}$ times larger. Ru demonstrates a larger intensity ratio than ballistic predictions (Figure 4.6b) which can be attributed to a larger compensation of $\sigma_{S}$ by $\sigma_{R}$ (Figure 4.7a), hinting towards larger retention channels. Additionally, the magnitude of $\sigma_{R}$ being lower than $\sigma_{S}$ for a given element, is consistent with previous reports at higher energies [18, 47]. 


\subsection{Conclusions}

We report the interaction of $\mathrm{N}_{2}^{+} / \mathrm{N}^{+}$ions with transition metal surfaces. Ballistic interactions were observed through sputter yields and data was obtained down to 50 $\mathrm{eV}$. Measured sputter yields were consistent within the binary collision approximation down to $50 \mathrm{eV}$ as evidenced from TRIDYN simulations. Estimation of retained nitrogen at the end of a fluence of $1 \times 10^{18} \mathrm{ion} / \mathrm{cm}^{2}$ from TRIDYN were performed assuming a low (Mode 1) and high (Mode 2) reflection coefficient, leading to nitrogen retention up to a stoichiometric nitride and sub-stoichiometric nitride level, respectively. Using simulated depth profiles, XPS intensity ratios were calculated, which were in accord with experimental values. The nitrogen content simulated by TRIDYN was corroborated by XPS measurements by comparing nitrogen/metal intensity ratios. Chemical interactions inferred from metal $3 \mathrm{~d} / 4 \mathrm{f}$ core levels indicated that Mo and $\mathrm{W}$ formed stoichiometric nitrides of $\mathrm{Mo}_{2} \mathrm{~N}$ and $\mathrm{WN}$ respectively; while $\mathrm{Ru}$ and $\mathrm{Pd}$ showed sub-stoichiometric levels of nitrogen. The metal core lines for $\mathrm{Ru}$ and $\mathrm{Pd}$ did not significantly change and unique deconvolution to estimate stoichiometry could not be performed. However, in all cases, experimental N1s core spectral lines indicated that the implanted nitrogen formed a metal nitride. Most importantly, it was shown that nitrogen retention in transition metals is nearly constant within the energy range of 50-500 eV. This was explained using reaction cross-sections, $\sigma_{R}$ that increase with increasing sputter yield as a function of energy. The increase in reaction cross-sections can be explained by a longer projected range of ions with higher energy. Reaction cross-sections were found to vary between elements as: $\sigma_{R}(\mathrm{Ru}) \sim \sigma_{R}(\mathrm{Pd}) \ll \sigma_{R}(\mathrm{Mo})<\sigma_{R}(\mathrm{~W})$. The combination of sputter yields + XPS + TRIDYN simulations serves as a useful tool to assess nitrogen content and metal reactivity where deconvolution of spectra is not unique and detailed fluence dependent data is unavailable. 


\section{References}

[1] M. K. Pospieszalska and R. E. Johnson, Magnetospheric ion bombardment profiles of satellites: Europa and Dione, Icarus 78, 1 (1989).

[2] R. Keller, D. Cheng, R. DiGennaro, R. A. Gough, J. Greer, K. N. Leung, A. Ratti, J. Reijonen, R. W. Thomae, T. Schenkel, J. W. Staples, R. Yourd, A. Aleksandrov, M. P. Stockli, and R. W. Welton, Ion-source and low-energy beam-transport issues with the front-end systems for the spallation neutron source, Review of Scientific Instruments 73, 914 (2002).

[3] K. Dobes, V. Smejkal, T. Schäfer, and F. Aumayr, Interaction between seeding gas ions and nitrogen saturated tungsten surfaces, International Journal of Mass Spectrometry 365-366, 64 (2014).

[4] R. C. Wieggers, W. J. Goedheer, M. R. Akdim, F. Bijkerk, and P. A. Zegeling, A particle-in-cell plus Monte Carlo study of plasma-induced damage of normal incidence collector optics used in extreme ultraviolet lithography, Journal of Applied Physics 103 (2008), 10.1063/1.2829783.

[5] M. H. L. Van Der Velden, W. J. M. Brok, J. J. A. M. Van Der Mullen, W. J. Goedheer, and V. Banine, Particle-in-cell Monte Carlo simulations of an extreme ultraviolet radiation driven plasma, Physical Review E - Statistical, Nonlinear, and Soft Matter Physics 73, 1 (2006).

[6] T. Van De Ven, P. Reefman, C. De Meijere, R. Van Der Horst, M. Van Kampen, V. Banine, and J. Beckers, Ion energy distributions in highly transient euv induced plasma in hydrogen, Journal of Applied Physics 123, 063301 (2018).

[7] W. Möller and D. Güttler, Modeling of plasma-target interaction during reactive magnetron sputtering of TiN, Journal of Applied Physics 102 (2007), $10.1063 / 1.2800262$.

[8] U. Plank, G. Meisl, U. von Toussaint, T. Höschen, and W. Jacob, Study of the temperature-dependent nitrogen retention in tungsten surfaces using $X$-ray photoelectron spectroscopy, Nuclear Materials and Energy 17, 48 (2018).

[9] P. He, Z. Zhang, W. Xia, L. Shu, X. Ma, F. Gou, and K. Zhang, Compatibility between high-flux helium plasma irradiated molybdenum and liquid lithium, Journal of Nuclear Materials 509, 736 (2018).

[10] S. M. Wu, R. Van De Kruijs, E. Zoethout, and F. Bijkerk, Sputtering yields of Ru, Mo, and Si under low energy Ar+ bombardment, Journal of Applied Physics 106, 0 (2009).

[11] P. Phadke, J. M. Sturm, R. W. van de Kruijs, and F. Bijkerk, Near-threshold sputter yields of ruthenium under argon and nitrogen ion bombardment, Nuclear Instruments and Methods in Physics Research, Section B: Beam Interactions with Materials and Atoms 458, 169 (2019). 
[12] G. P. Glazunov, E. D. Volkov, D. I. Baron, A. P. Dolgiy, A. Konotopskiy, and A. Hassanein, Effect of low/high Hydrogen Recycling OPERATION on Palladium Sputtering under Steady State Plasma Impact, Physica Scripta T103, 89 (2003).

[13] I. Takagi, K. Moritani, and H. Moriyama, Asymmetric surface recombination of hydrogen on palladium exposed to plasma, Journal of Nuclear Materials 313316, 102 (2003).

[14] D. Van Vechten, G. Hubler, and E. Donovan, Characterization of a $3 \mathrm{~cm}$ Kaufman ion source with nitrogen feed gas, Vacuum 36, 841 (1986).

[15] B. Nichols and F. C. Witteborn, Measurements of resonant charge exchange cross sections in nitrogen and argon between 0.5 and $17 \mathrm{ev}$, Nasa, 1 (1966).

[16] E. Benes, Improved quartz crystal microbalance technique, Journal of Applied Physics 56, 608 (1984).

[17] L. Toth, Transition metal carbides and nitrides (Elsevier, 2014).

[18] H. Hu, Y. Fukuda, D. A. Baldwin, P. T. Murray, and J. W. Rabalais, Interactions of ion beams with surfaces: Dynamics of the reaction of $N 2+$ with rhenium, The Journal of Chemical Physics 72, 6158 (2003).

[19] W. Möller, W. Eckstein, and J. P. Biersack, Tridyn-binary collision simulation of atomic collisions and dynamic composition changes in solids, Computer Physics Communications 51, 355 (1988).

[20] W. D. Wilson, L. G. Haggmark, and J. P. Biersack, Calculations of nuclear stopping, ranges, and straggling in the low-energy region, Physical Review $\mathrm{B}$ 15, 2458 (1977).

[21] N. Bohr and J. Lindhard, Electron capture and loss by heavy ions penetrating through matter, Kgl. Danske Videnskab. Selsk. Mat.fys. Medd. 28 (1954).

[22] W. Möller, TRIDYN User Guide 2017, HZDR, .

[23] W. Möller, F. Besenbacher, and J. Bottiger, Saturation and isotope mixing during low-temperature implantations of hydrogen into metals, Applied Physics A Solids and Surfaces 27, 19 (1982).

[24] Z. Y. Chen, A. Bogaerts, D. Depla, and V. Ignatova, Dynamic Monte Carlo simulation for reactive sputtering of aluminium, Nuclear Instruments and Methods in Physics Research, Section B: Beam Interactions with Materials and Atoms 207, 415 (2003).

[25] D. A. King and M. G. Wells, Molecular beam investigation of adsorption kinetics on bulk metal targets: Nitrogen on tungsten, Surface Science 29, 454 (1972).

[26] D. A. King, Nitrogen adsorption on nickel and palladium films, Surface Science 9, 375 (1968). 
[27] A. A. Parry and J. A. Pryde, Adsorption of nitrogen and carbon monoxide on molybdenum, British Journal of Applied Physics 18, 329 (1967).

[28] L. R. Danielson, M. J. Dresser, E. E. Donaldson, and J. T. Dickinson, Adsorption and desorption of ammonia, hydrogen, and nitrogen on ruthenium (0001), Surface Science 71, 599 (1978).

[29] M. G. Moreno-Armenta, J. Diaz, A. Martinez-Ruiz, and G. Soto, Synthesis of cubic ruthenium nitride by reactive pulsed laser ablation, Journal of Physics and Chemistry of Solids 68, 1989 (2007).

[30] A. L. Ivanovskii, Platinum group metal nitrides and carbides: synthesis, properties and simulation, Russian Chemical Reviews 78, 303 (2009).

[31] G. Meisl, K. Schmid, O. Encke, T. Höschen, L. Gao, and C. Linsmeier, Implantation and erosion of nitrogen in tungsten, New Journal of Physics 16 (2014), 10.1088/1367-2630/16/9/093018.

[32] W. Eckstein, R. Dohmen, A. Mutzke, and R. Schneider, SDTrimSP version 5.00, IPP Report 12/08, Max- Planck-Institut für Plasmaphysik (Hrsg.) 12 (2007), http://edoc.mpg.de/display.epl? $\operatorname{mode}=$ doc\&id $=552734$.

[33] D. Briggs and M. P. Seah, Practical Surface Analysis, Auger and X-ray Photoelectron Spectroscopy, Practical Surface Analysis (Wiley, 1990).

[34] J. H. Scofield, Hartree-Slater subshell photoionization cross-sections at 1254 and $1487 \mathrm{eV}$, Journal of Electron Spectroscopy and Related Phenomena 8, 129 (1976).

[35] J. J. Yeh and I. Lindau, Atomic subshell photoionization cross sections and asymmetry parameters: $1<Z<103$, Atomic Data and Nuclear Data Tables 32, 1 (1985).

[36] W. H. Gries, A universal predictive equation for the inelastic mean free pathlengths of $x$-ray photoelectrons and auger electrons, Surface and Interface Analysis 24, 38 (1996).

[37] E. Cattaruzza, G. Battaglin, P. Riello, D. Cristofori, and M. Tamisari, On the synthesis of a compound with positive enthalpy of formation: Zinc-blende-like RuN thin films obtained by rf-magnetron sputtering, Applied Surface Science 320, 863 (2014).

[38] Z. Wei, P. Grange, and B. Delmon, XPS and XRD studies of fresh and sulfided Mo2N, Applied Surface Science 135, 107 (2002).

[39] Y. G. Shen, Y. W. Mai, D. R. McKenzie, Q. C. Zhang, W. D. McFall, and W. E. McBride, Composition, residual stress, and structural properties of thin tungsten nitride films deposited by reactive magnetron sputtering, Journal of Applied Physics 88, 1380 (2000). 
[40] G. M. Veith, A. R. Lupini, L. Baggetto, J. F. Browning, J. K. Keum, A. Villa, L. Prati, A. B. Papandrew, G. A. Goenaga, D. R. Mullins, S. E. Bullock, and N. J. Dudney, Evidence for the formation of nitrogen-rich platinum and palladium nitride nanoparticles, Chemistry of Materials 25, 4936 (2013).

[41] G. Greczynski, D. Primetzhofer, J. Lu, and L. Hultman, Core-level spectra and binding energies of transition metal nitrides by non-destructive x-ray photoelectron spectroscopy through capping layers, Applied Surface Science 396, 347 (2017).

[42] M. A. Makeev and A. L. Barabási, Effect of surface morphology on the sputtering yields. I. Ion sputtering from self-affine surfaces, Nuclear Instruments and Methods in Physics Research, Section B: Beam Interactions with Materials and Atoms 222, 316 (2004).

[43] I. Bizyukov, K. Krieger, N. Azarenkov, and U. V. Toussaint, Relevance of surface roughness to tungsten sputtering and carbon implantation, Journal of Applied Physics 100 (2006), 10.1063/1.2400393.

[44] H. D. Hagstrum, Theory of auger ejection of electrons from metals by ions, Physical Review 96, 336 (1954).

[45] N. Tolk, Inelastic Ion-Surface Collisions (Elsevier Science, 2012).

[46] T. Darko, D. A. Baldwin, N. Shamir, J. W. Rabalais, and P. Hochmann, Reactions of homonuclear diatomic ions with metal surfaces. I. Model for $X+$ 2 beam-surface reactions in the low kinetic energy near threshold region, The Journal of Chemical Physics 76, 6408 (1982).

[47] H. K. Hu, P. T. Murray, Y. Fukuda, and J. W. Rabalais, Absolute cross sections for beam-surface reactions: $N 2+$ on Ti from 0.25 to $3.0 \mathrm{keV}$ kinetic energy, The Journal of Chemical Physics 74, 2247 (1981). 


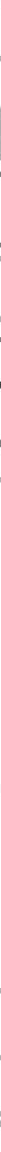

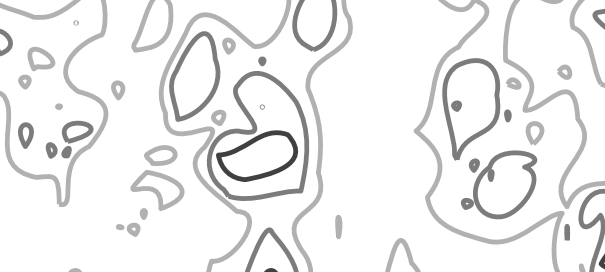

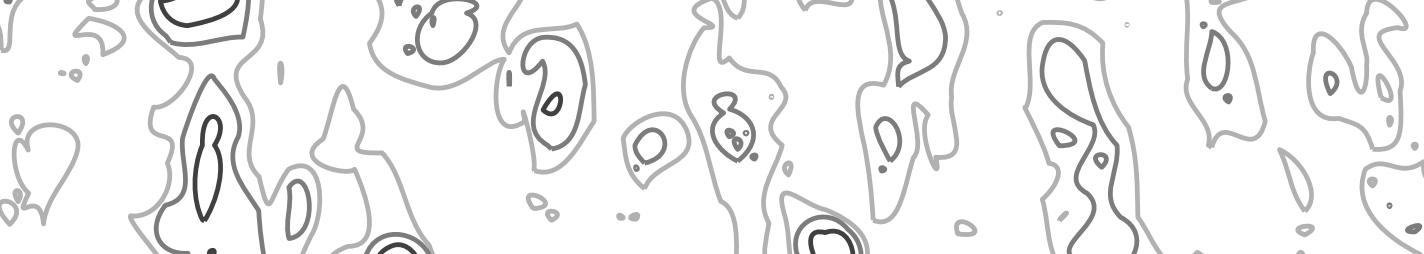

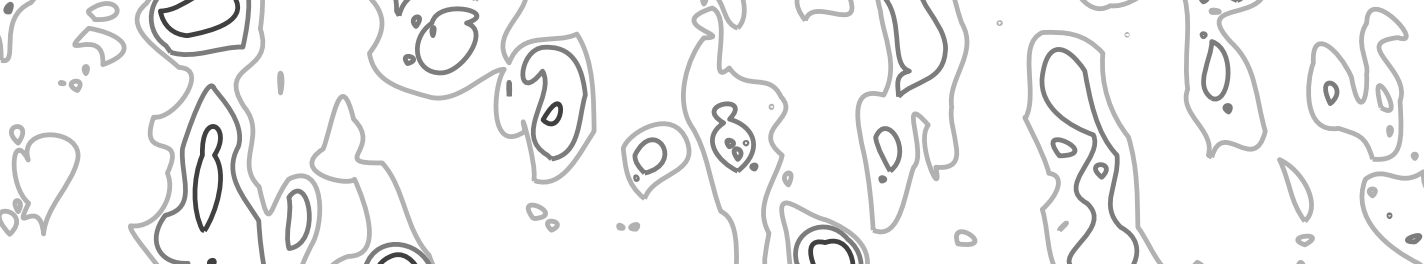

o.

$0.04<0$

30 ?

$3 \cdot 0$

.000 cos

(a) (a)

o.

in 2

o)

- ove

$\left.\operatorname{lin}^{0}: 3\right\}$

(3) 0.0 .50

2.

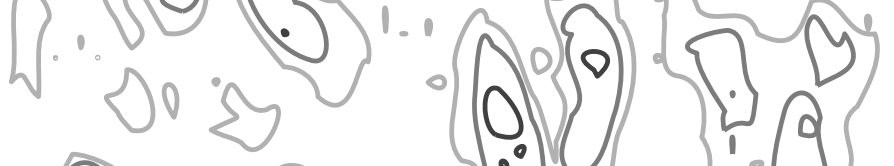

$\Rightarrow$ is

(.).

$\because 20)$

.

(o) $a^{\circ}$

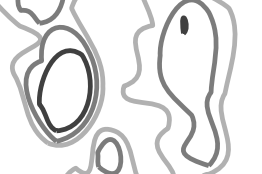

S. 300 of 5 : $\therefore=0$ 


\section{5}

\section{SPUTTERING AND OXIDATION: RADIATION ENHANCED DIFFUSION}

This isn't right. This isn't even wrong.

Wolfgang Pauli

Transition metals used in semiconductor, photo-lithography and fusion applications interact with low energy oxygen ions. Understanding erosion, the nature of the formed oxide and depth of oxygen transport is necessary in mitigating unexpected performance of sensors, optics or plasma facing components. Oxide formation is governed by both the ion-target combination and the incident ion energy. We study the interaction of the transition metals molybdenum, ruthenium, palladium and tungsten, with oxygen ions in the energy region of $50-500 \mathrm{eV}$. Near-threshold sputtering of metals was experimentally measured and compared to predictions by the Monte Carlo code TRIDYN. Compositional changes and oxide thicknesses following sputtering were measured using Angle resolved X-Ray photoelectron spectroscopy and subsequently compared to limiting oxide formed by atomic oxygen exposures. Sputter yields in some cases (ruthenium) were found to be sensitive to ion beam impurities such as ozone ( $<1 \%$ of background gas) leading to chemical sputtering. Ion induced oxide thicknesses (for molybdenum and tungsten) were found to be larger than those predicted by ballistic transport where sputtering is balanced by implantation. It is hypothesized that radiation enhanced diffusion of free oxygen leads to thicker oxide films at low ion energies. 


\subsection{Introduction}

Metal oxides are of interest to a wide range of applications, from semiconductor manufacturing, to extreme ultraviolet (XUV) lithography optics and fusion research. Oxygen ion and plasma interactions with metals have been particularly relevant in these fields [1-4]. On one hand, controlled interactions, in semiconductor manufacturing and thin film deposition applications, can assist in selective, anisotropic oxidation of metal films or deposition of oxide layers. On the other hand, uncontrolled ion-target surface interactions, in photo-lithography and fusion applications, can lead to material loss $[5,6]$ and undesirable oxidation of plasma facing components [4, 7-11]. In either case, insights into the interaction processes, namely, sputtering and oxide formation, help offer better control over the evolution of surface (and sub-surface) composition.

Low energy $(<500 \mathrm{eV})$ oxygen ions are of particular interest as sputter processes can depend on ion-surface interaction times [12]. Further, oxidation can be influenced by an increased sticking probability at low energies in which case an extrapolation from high energy data cannot be made. In this regard, studies on low kinetic energy oxygen ion induced sputtering and oxidation of metals are few and scattered. Initial reports by Hechtl and Bohdansky [13] study the sputter yields for molybdenum under $\mathrm{O}^{+}$ion bombardment down to $100 \mathrm{eV}$. This was complemented also by Hechtl et al. [14] for tungsten. While sputter yields were reported, they mention neither the depth of ion distribution nor a sputter threshold. The oxidation states for molybdenum and tungsten [15], nickel [16] and chromium [17] were studied as a function of ion fluence using $1 \mathrm{keV} \mathrm{O}_{2}^{+}$ions. However, the depth of oxygen penetration and energy dependence was not discussed in detail. Given the disparities in metals, energy ranges, ion species and transport processes studied so far, a complete map of information regarding sputtering and oxidation of transition metals at low ion energies is difficult to construct for any particular metal.

We aim to provide a more complete dataset in the energy region of $50-500 \mathrm{eV}$, and discuss the processes involved in low energy oxygen ion induced sputtering and oxidation of transition metals relevant for the applications outlined above. Sputter yields were measured for molybdenum, tungsten, ruthenium and palladium at steady state, where implantation of oxygen (and compound formation) is balanced by sputtering of metal and implanted oxygen. In the case of ruthenium and palladium, yields are reported for the first time using oxygen ions. Sputter yields are then compared to the predictions by the dynamic Monte Carlo code TRIDYN and discrepancies between experiments and simulations are briefly discussed. Oxygen transport within the metal targets is then studied in the same energy range by measuring oxide thickness. Additionally, the influence of particle kinetic energy on oxide thickness is investigated by comparing results of ion exposed samples to samples exposed to atomic oxygen species at thermal energies. For ion exposed samples, in most cases the depth of oxygen penetration was seen to be under-estimated by TRIDYN, which only accounts for ballistic transport. This indicates existence of diffusion mechanisms, which were then externally modeled using existing TRIDYN outputs. Thus, radiation enhanced diffusion mechanisms are hypothesized to act in transport of oxygen deeper into the target. 


\subsection{Experimental Methods}

The ion exposure facility used in this work has been described in detail in previous publications $[6,18]$ as well as chapters 3 and 4 . The setup is comprised of a vacuum chamber and ion source assembly baked out to a base pressure of $1 \times 10^{-8}$ mbar. A $15 \mathrm{~cm}$ DC Kaufman ion source (Veeco Instruments) was supplied with pure oxygen feed gas to generate oxygen ions. The source was retrofitted with a Faraday cup which also served as a retarding field energy analyzer $(\mathrm{FC}+\mathrm{RFEA})$. The FC+RFEA and metal film samples were mounted at a distance of $7 \mathrm{~cm}$ from the source exit. Ion energies were calibrated using the $\mathrm{FC}+\mathrm{RFEA}$ operating in energy analyzer mode. The beam of oxygen ions created at a discharge voltage of $80 \mathrm{~V}$ was mono-energetic with a FWHM of $\sim 10 \mathrm{eV}$ over the energy range of $50-500 \mathrm{eV}$ used in this work. The composition of the beam in terms of atomic and molecular ion species was not explicitly measured. However, comparing reported composition from similar Kaufman-type sources, the beam is expected to comprise of $80-90 \% \mathrm{O}_{2}^{+}$and $10-$ $20 \% \mathrm{O}^{+}$ions with $<1 \%$ of $\mathrm{O}_{3}^{+}$impurities.

Ion exposures were carried out on metal films. Two kinds of samples were produced using magnetron sputter deposition: $400 \mathrm{~nm}$ (thick) films grown on a quartz crystal microbalance (QCM) which we shall refer to as M-QCM to differentiate them from the mass measuring crystal itself; and $80 \mathrm{~nm}$ (thin) films grown on silicon substrates (MSi). Both M-QCM and MSi samples were mounted at the same radial distance from the center of the source radial axis as the FC + RFEA. This allowed simultaneous ion exposure and flux monitoring. QCM and MSi experiments were carried out separately due to the nature of the experiments, where M-QCM samples allowed for multiple variations in ion energy in a single experiment while M-Si samples were exposed to a single energy for a fixed fluence.

For QCM irradiation to $\mathrm{O}_{2}^{+} / \mathrm{O}^{+}$ions, the experiments comprised of alternating steps of $300 \mathrm{eV} \mathrm{Ar}{ }^{+}$exposure to clean the layer, followed by $\mathrm{O}_{2}^{+} / \mathrm{O}^{+}$exposure steps to determine the sputter yield. The $\mathrm{Ar}^{+}$exposure step was introduced to remove adsorbed impurities, native oxides and oxides formed by the $\mathrm{O}_{2}^{+} / \mathrm{O}^{+}$ion bombardment on the M-QCM film surface from a previous exposure. The energies of the $\mathrm{O}_{2}^{+} / \mathrm{O}^{+}$exposure steps were varied randomly to avoid systematic errors. The frequency response from the QCM was constantly monitored and converted to a thickness value through the Z-match method [6, 18]. The QCM signal in the initial stage of $\mathrm{O}_{2}^{+} / \mathrm{O}^{+}$exposure showed a non-linear response due to incorporation of oxygen and thermal loads on the QCM oscillator from the ion and thermal flux from the ion source. After this initial stage $\left(\sim 1 \times 10^{16}\right.$ ions $\left./ \mathrm{cm}^{2}\right)$, the QCM shows a linear response consistent with material removal, and ion exposures were carried out up to a fluence of $1 \times 10^{18}$ ions $/ \mathrm{cm}^{2}$, where the high fluence was chosen to obtain good statistics at lower energies while ensuring the impingement processes reached a steady state. Exposure of $\mathrm{Pd}, \mathrm{Mo}$ and $\mathrm{W}$ to $<60 \mathrm{eV}$ ions resulted in an increase of the measured thickness value during ion exposure due to oxidation of the metal dominating sputtering and/or deposition of source grid material on the QCM. The source grids, made of molybdenum, sputter at low ion energies due to the inefficient lensing of the ion beam and sputtered grid material can redeposit onto the MSi and M-QCM samples as evidenced from post exposure XPS measurements. This 
deposition is negligible at energies $\geq 60 \mathrm{eV}$ as no Mo peaks were found in postexposure XPS. MSi samples were exposed to a single $\mathrm{Ar}^{+}$cleaning step followed by an $\mathrm{O}_{2}^{+} / \mathrm{O}^{+}$exposure at a single energy to fluence of the order of $1 \times 10^{18}$ ions $/ \mathrm{cm}^{2}$.

Errors in estimated sputter yields from M-QCM samples arise from drifts in ion current $(<2 \%)$ and calculation of the etch rates $(\sim 10 \%-20 \%$, decreasing with increasing ion energy) from the frequency response. Corrections to the frequency response due to density changes from compound formation were not performed. Finally, charge exchange neutralization of $\mathrm{O}_{2}^{+}$ions lead to errors of $\sim 5 \%$ assuming cross-sections in the order of those of $\mathrm{N}_{2}^{+}$[19]. In total, an error of upto $40 \%$ is expected, which decreases with increasing ion energy.

To compare reactivity of oxygen ions and the role of energy in the oxidation process, MSi samples were exposed to neutral atomic oxygen (at-O) in a separate facility. The direct use of at-O eliminates the dissociation step of $\mathrm{O}_{2}$ (which occurs upon impact with the surface for molecular oxygen ions) and maintains a high concentration of strong electron acceptors at the surface [20, 21]. With this, a strong electric field $E_{\mathrm{M}}$, generated by the oxygen and metal interactions, promotes positive metal ion diffusion to react with the adsorbed oxygen, forming an oxide layer at the surface. $E_{\mathrm{M}}$ is determined as $E_{\mathrm{M}}=-V_{\mathrm{M}} / L(t) . V_{\mathrm{M}}$ is the Mott potential and $L(t)$ is the formed oxide thickness. The oxide growth will only cease when the field generated by the Mott-potential is insufficient to act as driving force for ionic diffusion through the oxide. This also results in a laterally uniform oxide, as defects and irregularities at atomic and nanoscales (responsible for molecular dissociation) will not play an important role in the oxidation process [22, 23]. Neutral at-O were generated by a Specs MPS-ECR mini plasma source, at an atomic oxygen flow in the order of $1 \times 10^{15}$ atoms $/ \mathrm{cm}^{2} / \mathrm{s}$ (partial pressure of $1 \times 10^{-4} \mathrm{mbar}$ - background $\mathrm{O}_{2}$ ). The samples were exposed at room temperature for 240 minutes, which was sufficient to saturate the oxide thickness [21].

MSi samples exposed to $\mathrm{O}_{2}^{+} / \mathrm{O}^{+}$ions and at-O were stored under a high vacuum of $1 \times 10^{-7}$ mbar before being transferred through ambient pressures for ARXPS analysis. MSi samples were exposed to a maximum of 20 minutes in ambient pressures prior to analysis. AR-XPS measurements were acquired using a ThermoFisher Theta probe angle resolved spectrometer with monochromatic Al-K $\alpha$ (1486 $\mathrm{eV}$ ) radiation and $400 \mu \mathrm{m}$ spot size. Angle resolved measurements were obtained for all MSi samples, however, for brevity AR-XPS spectra reported in the present work are limited to a take-off angle of $34.25^{\circ}$ (corresponding to the highest probing depth available).

\subsection{Model and Simulations}

\subsubsection{TRIDYN simulations of sputter yields and depth profiles}

Sputter yields obtained from frequency changes of the QCM were compared to theoretical predictions of the Monte Carlo code TRIDYN [24]. TRIDYN tracks asymptotic trajectories of atomic collisions under the binary collision approximation. TRIDYN accounts for dynamic changes in surface composition during the sputtering process and has been successfully applied to study effects of target poi- 
soning [25], reactive sputtering [26] and ion implantation [27].

Simulations were performed on an $80 \mathrm{~nm}$ metal target divided into 400 lamellae, for a fluence of $2 \times 10^{18}$ ions $/ \mathrm{cm}^{2}$. The simulated beam comprised of $\mathrm{O}_{2}^{+}$ions with $20 \% \mathrm{O}^{+}$impurity. Molecular $\mathrm{O}_{2}^{+}$species having an energy $E_{0}$ are assumed to neutralize near the metal surface and dissociate under collision, resulting in $2 \mathrm{O}$ particles, each having energy $0.5 \times E_{0}$, interacting with the target. For comparing simulations and experiments, all text and graphs in this work always refer to the initial kinetic energy $E_{0}$. The surface binding energy (SBE) is defined by a uniform surface potential which determines whether an atom from a cascade induced by incoming ions, whose momentum vector points in the direction of the surface-vacuum interface, leaves the surface and is consequently sputtered. The choice of SBE is important in determining the sputter yield and SBE values required by TRIDYN are chosen to be equal to the sublimation energy for the pure metal $\left(U_{\mathrm{S}}\right)$. The binding energy of the compound $\left(S B E_{M e-O}\right)$ was chosen through thermodynamic considerations as $[24,26,28]$ :

$$
S B E_{M e-O}=\frac{1}{2} U_{S}+\frac{n+m}{2 n m} \Delta H_{f}+\frac{n+m}{4 n} \Delta H_{d}^{O}
$$

where $n$ and $m$ are the stoichiometries of the formed oxide of type $\mathrm{Me}_{n} \mathrm{O}_{m} ; \Delta H_{f}$ is the enthalpy of formation of the compound and $\Delta H_{d}^{O}$ is the dissociation energy of the oxygen molecule $(\sim 5.15 \mathrm{eV})$. To mimic compound formation, an oxygen saturation limit $\left(O_{\text {sat }}\right)$ was set corresponding to the $\mathrm{O}$ atomic fraction in the most abundant bulk metal oxide. Oxygen accumulating above $\mathrm{O}_{\text {sat }}$ is considered to be reflected and is removed from the simulation. A summary of parameters used is listed in Table 5.1.

We carry out simulations under two conditions: i) a saturable oxygen uptake accounting for only collision cascades modified by implanted oxygen; and ii) a saturable oxygen uptake accounting for modified collision cascades with an additional surface binding energy dependent on oxygen concentration according to equation 5.1. All simulations were carried out using the dynamic mode. The density of oxygen implanted in the material was allowed to a maximum of the fraction in the bulk

Table 5.1: Parameters used for TRIDYN simulations. Negative values of $\Delta H_{f}^{O}$ indicate exothermic reactions for oxide formation.

\section{Parameters}

\begin{tabular}{lllllll}
\hline \hline Element & $\begin{array}{l}\text { Saturated } \\
\text { Oxide }\end{array}$ & $\Delta H_{f}^{O}(\mathrm{eV})$ & $\begin{array}{l}\text { Oxide } \\
\text { density } \\
\left(\mathrm{g} / \mathrm{cm}^{3}\right)\end{array}$ & $\begin{array}{l}\text { Saturation } \\
\text { Atomic }\end{array}$ & $\begin{array}{l}U_{\mathrm{S}}(\mathrm{eV}) \\
\begin{array}{l}\text { Fraction } \\
\left(O_{\text {sat }}\right)\end{array}\end{array}$ & $\begin{array}{l}S B E_{M e-O} \\
(\mathrm{eV})\end{array}$ \\
\hline $\mathrm{Mo}$ & $\mathrm{MoO}_{3}$ & -7.82 & 4.69 & 0.75 & 6.82 & 13.8 \\
$\mathrm{Ru}$ & $\mathrm{RuO}_{2}$ & -3.25 & 6.97 & 0.66 & 6.74 & 9.67 \\
$\mathrm{Pd}$ & $\mathrm{PdO}$ & -1.18 & 8.70 & 0.5 & 3.89 & 5.71 \\
$\mathrm{~W}$ & $\mathrm{WO}_{3}$ & -8.71 & 7.16 & 0.75 & 8.90 & 15.3 \\
\hline
\end{tabular}


value.

\subsubsection{Multi-Overlayer Model}

AR-XPS measurements were deconvolved to separate metal core level peaks from the metal oxide components. Subsequently, the peaks were used to determine thickness of the oxide layer formed after ion bombardment using the multi-overlayer model. This model relates the XPS peak intensity ratios from substrate and thin films to a thickness via [29]:

$$
d=\lambda_{i} \cos (\theta) \log \left[1+\frac{R}{R_{\infty}}\right]
$$

where $\lambda_{i}$ is the effective attenuation length of the photoelectrons calculated using the approach of Cumpson and Seah [30]. A list of attenuation lengths used is provided in table 5.2. $\theta$ is the photoelectron take-off angle with respect to the surface normal, $R$ is the ratio of the measured film XPS peak intensity to the XPS peak from the substrate layer underneath. $R_{\infty}$ is the (hypothetical) ratio for bulk film and substrate intensities, which accounts for sensitivity factors. The thin film thickness, $d$, can be obtained by a least square fitting of equation 5.2 to intensity ratios as a function of $\theta$. This is particularly useful for low energy implantation studies where the depth of data acquisition is comparable to the region of ion influence. We rely on the multi-overlayer calculator in the commercially available Thermo-Avantage software (v.5.952, (c) 1999-2014 Thermo Fisher Scientific) for AR-XPS data processing.

Table 5.2: Attenuation lengths of metal $\left(\lambda_{M}\right)$ and metal-oxide $\left(\lambda_{M O}\right)$ used in estimating oxide thickness using the Multi-overlayer model in equation 5.2 using the Cumpson-Seah equation [30].

\begin{tabular}{ccc}
\hline \multicolumn{3}{c}{ Parameters } \\
\hline \hline Element & $\lambda_{\mathrm{M}}(\mathrm{nm})$ & $\lambda_{\mathrm{MO}}(\mathrm{nm})$ \\
\hline $\mathrm{Mo}$ & 1.50 & 1.99 \\
$\mathrm{Ru}$ & 1.35 & 1.62 \\
$\mathrm{Pd}$ & 1.32 & 1.49 \\
$\mathrm{~W}$ & 1.35 & 1.95 \\
\hline
\end{tabular}

\subsection{Results and Discussion}

\subsubsection{Sputter Yields from QCM Measurements}

The sputter yields measured from M-QCM films at steady state correspond to the (partial) yield of the metal, since at steady state the mass loss due to sputtering of $\mathrm{O}$ atoms from the formed oxide film is compensated by implantation of new oxygen atoms in the film. Measured yields are shown in Figure 5.1 along with sputter yields from TRIDYN simulations performed according to Section 5.3.1. Reference data for $\mathrm{O}^{+}$ion irradiation were available for molybdenum [13] and tungsten [14] and are also included. 
a) $\mathrm{Pd}$

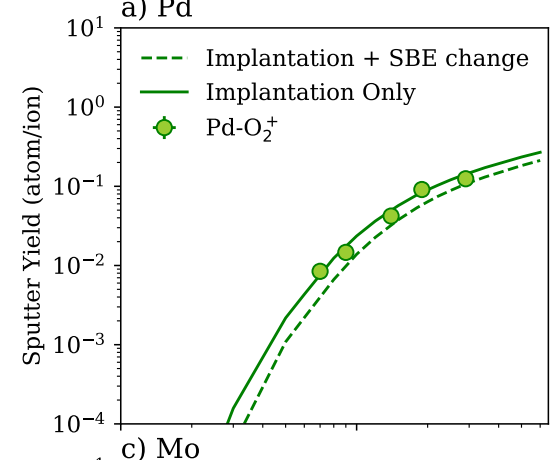

b) $\mathrm{Ru}$

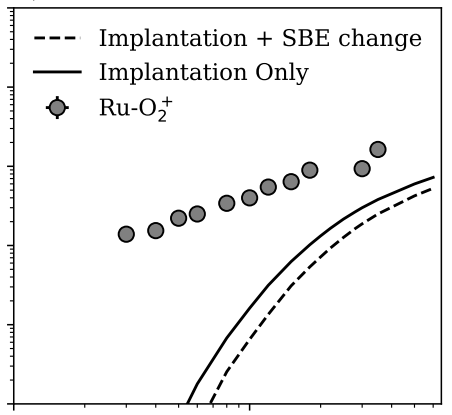

d) $\mathrm{W}$

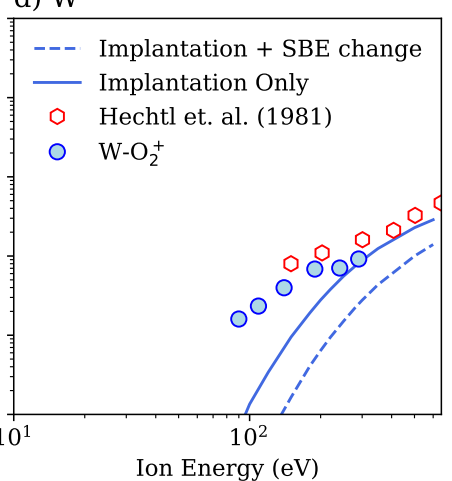

Figure 5.1: Sputter yields of a) palladium; b) ruthenium; c) molybdenum and d) tungsten obtained from quartz microbalance measurements. Experimental data (circles) are plotted against TRIDYN simulations that account for cascade modification by oxygen implantation only (solid line) and implantation with SBE changes (dashed line). Literature values for $\mathrm{O}^{+}$sputter yields for Mo [13] and $\mathrm{W}[14]$ are also included.

As mentioned in Section 5.2, below $60 \mathrm{eV}$ a net negative yield is obtained for $\mathrm{Pd}$, Mo and W, due to deposition of material on the QCM and these data are discarded in the present report. Sputter yields from palladium (Figure 5.1a) are well described by TRIDYN's binary collision approximation. A change in surface binding energy due to compound formation does not deviate significantly from implantation induced modification of collision cascades. The calculated $S B E_{M e-O}=$ $5.6 \mathrm{eV}$, which is $\sim 45 \%$ larger than the pure metal, which leads to a reduction in the predicted sputter yield by as much as a factor 2 at $70 \mathrm{eV}$. Given the error in the data, both models provide reasonable estimates of the sputter yields although at energies below $100 \mathrm{eV}$ the model without modifications to the SBE seems to describe the data better. Sputtering by energies below $70 \mathrm{eV}$ was not measurable, however, a threshold energy $\left(E_{\mathrm{th}}\right)$ of $22 \pm 3 \mathrm{eV}$ is predicted from simulations.

Ruthenium demonstrates a unique behavior with respect to oxygen ion bombardment (Figure 5.1b) compared to the other transition metals studied here. The measured sputter yields do not behave according to predictions by standard binary collision approximations and energy transfer models of TRIDYN. The sputter yields 
remain as high as 0.02 atoms per ion even down to $30 \mathrm{eV}$, even in the presence of grid material deposition that led to negative sputter yields for the other materials. The results suggest a mechanism other than ballistic energy transfer through binary collisions. Sublimation or production of volatile species can enhance sputter yields through 'chemical' processes. Ruthenium is known to have a single volatile oxide: $\mathrm{RuO}_{4}$, which, if produced under ion bombardment would explain the behavior observed here. We shall discuss this possibility, along with other pertinent factors in the following section.

Molybdenum sputter yields (Figure 5.1c) were measured successfully down to 70 $\mathrm{eV}$. Comparison to literature reports on sputtering of molybdenum by $\mathrm{O}^{+}$ions [13] shows that reported yields are consistent with values measured in the present work. Yao et. al [12] have shown that molecular and atomic ion species vary in sputter efficiency due to differences in energy transferred to the metal lattice. However, these differences appear at energies $<100 \mathrm{eV} /$ atom for oxygen. Available literature is limited to energies above $100 \mathrm{eV} /$ atom, and a comparable yield is expected and subsequently observed. Simulations from TRIDYN qualitatively mimic the sputter process with better agreement at higher energies and a steady divergence from predictions upon approaching the sputter threshold. Measured yields being higher than theoretical predictions could be due to changes in roughness of the bombarded film, structure, thickness and stress of the formed oxide film. The experimentally observed sputter threshold of $70 \mathrm{eV}$ lies at the edge of the threshold of $60 \pm 10 \mathrm{eV}$ obtained from averaging the two simulation results.

Available data for tungsten [14] from $\mathrm{O}^{+}$ion sputtering is consistent within error to yields obtained by QCM measurements (Figure 5.1d). The comparison between $\mathrm{O}^{+}$and $\mathrm{O}_{2}^{+}$is possible as mentioned previously. Experiments below $90 \mathrm{eV}$ resulted in a frequency response indicating thermal drift; and mass gain below 60 $\mathrm{eV}$. Although errors in low energy yield values are large due to influence of thermal drift of the QCM and more generally a low sputter rate, the experimental values are significantly larger than simulations. Both experimental datasets in Figure 5.1d for tungsten begin to deviate from TRIDYN predictions at energies $<250 \mathrm{eV}$. The sputter yields show a near linear trend on a log-log scale. Such a behavior is akin to chemical sputtering of targets like carbon under oxygen or hydrogen ion bombardment [31].

Given that certain materials (palladium) behave consistently with simulations while others show discrepancies at low energies, we shall consider the experimental factors that can potentially skew measurements of yields in the following section.

\section{Factors Influencing Sputter Yields}

Experimental measurements of sputter yields can be affected by uncertainties in beam diagnostics or experimental design. We have previously considered factors that play a role in the uncertainty of measured values, but there exist factors that can linearly or non-linearly skew measurements such as: sample charging, presence of atomic impurities, molecular effects dictated by ion-target interaction times, and chemical effects. Here, we shall briefly discuss each effect and consider the impact on the measured sputter yields.

Effects of charging of the formed oxide films can be ruled out in the present sput- 
ter yield measurements as the samples and QCMs were grounded and the formed oxide is relatively shallow (Section 5.4.3).

Sputter yields can increase due to the presence of atomic impurities. As $\mathrm{O}^{+}$ions do not dissociate, they maintain an energy larger by a factor 2 than $\mathrm{O}_{2}^{+}$ion species. Their effect on sputter yields would then be larger near the sputter threshold where $\mathrm{O}_{2}^{+}$ions dissociate and possess energies below $E_{t h}$ while $\mathrm{O}^{+}$ions can still have an energy larger than $E_{t h}$ and sputter target material. The source was not directly characterized for beam composition, nor was the beam mass-filtered due to geometric constraints, therefore, the presence of higher concentrations $(>20 \%)$ of $\mathrm{O}^{+}$ions cannot be excluded. However, due to the ballistic nature of the process involved, we expect TRIDYN to predict the yield under higher $\mathrm{O}^{+}$ion concentrations. Subsequently, TRIDYN simulations were performed for all materials by defining ion beams with a $40 \% \mathrm{O}^{+}$ion fraction. For an $\mathrm{O}^{+}$fraction of $40 \%$ under conditions of only incorporation of oxygen without binding energy variation, the yields were larger by a factor 2 at $70 \mathrm{eV}$ in comparison to simulations with $20 \% \mathrm{O}^{+}$content. As the experiments were carried out for all elements under the same conditions, this impurity concentration would encompass all metals studied. This over-estimates the sputtering for palladium (by a factor 2), and still underestimates the sputtering for the remaining targets (by at least a factor 2). Given the larger errors at the lower energies, it can be posited that $\mathrm{O}^{+}$impurities may be larger than the estimate of $20 \%$ (by at most a factor 2) for all experiments and influence the sputter yields. However, the enhancement observed in simulations by a larger impurity concentration does not fully explain the yields at low energies which remain as high as $3 \times$ and $8 \times$ for Mo and W, respectively in comparison to simulations.

It was shown by Yao [12] that molecular effects can play a role for $\mathrm{O}_{2}^{+}$below 100 $\mathrm{eV}$, where the interaction time of an ion-atom collision is larger than the vibrational frequencies of the $\mathrm{O}-\mathrm{O}$ bond. In such cases, the incoming $\mathrm{O}_{2}^{+}$ions can behave as a rigid body with $2 \times$ the mass of $\mathrm{O}$ and thus show an increased sputter yield in comparison to $\mathrm{O}^{+}$. This behavior is not modeled in our present simulations and would be an active phenomenon in the experiments for all metals studied. We can assess the magnitude of this effect as per Yao's considerations. From a ballistic standpoint, the yield enhancement is dictated by the effective energy transferred by the molecule to the target. The bounds can lie between the energy transfer of a single atom mass (as TRIDYN assumes) and a rigid molecule colliding with the mass of 2 atoms (the unified atom limit). It is described as [12]:

$$
\frac{\Delta E_{\text {molecule }}}{\Delta E_{\text {atom }}}=\frac{2\left(m_{\text {ion }}+M_{\text {target }}\right)^{2}}{\left(2 m_{\text {ion }}+M_{\text {target }}\right)^{2}}
$$

For our targets studied, this approximation leads to an enhancement in sputtering by $\sim 1.5 \times$ for $\mathrm{Mo}, \mathrm{Ru}$ and $\mathrm{Pd}$, and $1.71 \times$ for $\mathrm{W}$ when bombarded by $\mathrm{O}_{2}^{+}$over $\mathrm{O}^{+}$. The enhancement factor is responsible for effectively rescaling the energy axis by the appropriate ratio. While this may not necessarily describe the exact process, it describes the limits to which an incoming molecular ion specie can enhance the sputter yield. Thus, under the limits of a unified atom and a rigid bond, the experimental data may deviate (on the abscissa) by as much as the enhancement factor for energies below $100 \mathrm{eV}$. 


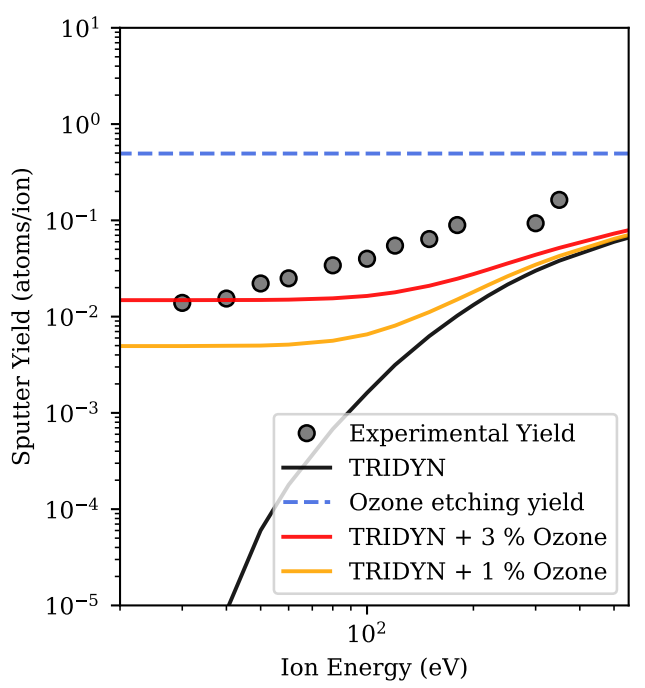

Figure 5.2: Ruthenium sputter yields as a function of incident ion energy. Experiments (circles) are much higher than TRIDYN predictions (black). The blue dashed line represents the assumed chemical sputter yield in the hypothetical case of a pure $\mathrm{O}_{3}$ molecular beam. Weighted averages of TRIDYN yields and an ozone impurity in the beam qualitatively describe the sputter yield observed. Note, $3 \%$ of thermal ozone relative to the ion flux corresponds to $0.1 \%$ of ozone in the background gas.

Volatile oxide formation can result in chemical etching and thus a higher sputter yield. Volatile oxides of molybdenum and tungsten exist, however, literature reports are confined to studies at elevated temperatures [32-34]. Palladium does not form volatile oxides which is consistent with findings in the present study. As mentioned in the previous section, volatile species of ruthenium may form, leading to a chemically enhanced sputter yield. In some detail, ruthenium exhibits a tendency for volatile $\mathrm{RuO}_{4}$ formation under thermal $\mathrm{O}_{3}$ interactions [35]. Generation of ozone occurs in oxygen plasmas from 3 body collisions between excited at-O and oxygen or ozone molecules [36, 37]. While dedicated corona discharges can efficiently generate ozone, in most industrial ion sources, ozone concentration is low. It is expected that a fraction of $<1 \%$ of the gas species in this experiment consist of $\mathrm{O}_{3}$ [38]. The etching of ruthenium by ozone proceeds as [39]: $\mathrm{Ru}+2 \mathrm{O}_{3} \rightarrow \mathrm{RuO}_{4}$ (gas) $+\mathrm{O}_{2}$. The volatile $\mathrm{RuO}_{4}$ readily decomposes to $\mathrm{RuO}_{2}$ and is difficult to detect without dedicated experiments [35]. Crudely, assuming the $\mathrm{O}_{3}$ etching proceeds in a similar manner and is independent on energy due to its chemical nature for a fixed temperature $(300 \mathrm{~K})$ of experiments, we can assign a yield value of 0.5 atoms $/ \mathrm{O}_{3}$ molecule. A weighted average of the ozone yield with the yield predicted by TRIDYN for slight variations in $\mathrm{O}_{3}^{+}$impurity fraction is shown in Figure 2 .

It is evident from the calculated yields that the effect of adding a chemical component to sputter yield simulations is important at energies below a few hundred $\mathrm{eV}$ and becomes negligible at higher energies. At energies below $100 \mathrm{eV}$ the chemical sputtering dominates and a saturation of the yield occurs which is proportional to 
the concentration of the ozone impurity. The presence of ozone up to $\sim 3 \%$ of the incident ion flux could partially explain the high sputter yields at low energies for ruthenium, with the remainder of deviations attributed to variations of the extracted ion flux or ion impact enhanced chemical kinetics [40].

For the above assumptions to be valid, ozone must form within the plasma, which is not un-realistic [36], and reach the target as neutral molecules at near thermal energies to prevent dissociation upon impact. The flux of ozone should thus result from background gas of the plasma and would therefore remain constant at all ion energies. In the calculations above, however, a weighted average of $\mathrm{O}_{3}$ and $\mathrm{O}_{2}^{+} / \mathrm{O}^{+}$ etching is calculated under the assumption that $\mathrm{O}_{3}$ species form a specific fraction of the ion beam flux (without consideration of background gas species).

To compare with the estimate of $0.1 \% \mathrm{O}_{3}$ background gas generated by DC Kaufman type ion sources [38], let us assume the flux generated by an ion source is constant at all energies and equal to $1 \times 10^{15}$ ions $/ \mathrm{cm}^{2} \mathrm{~s}$. Operating at a background pressure of $1 \times 10^{-4}$ mbar, of which $0.1 \%$ partial pressure is $\mathrm{O}_{3}$, the flux of $\mathrm{O}_{3}$ molecules neutral particles is then $2.2 \times 10^{13}$ molecules $/ \mathrm{cm}^{2} \mathrm{~s}$. A flux of thermal $\mathrm{O}_{3}$ equal to $3 \%$ of the ion flux (as assumed in Figure 2), would correspond to $3 \times 10^{13}$ ozone $/ \mathrm{cm}^{2}$ s. Thus, a $0.1 \%$ of $\mathrm{O}_{3}$ in the background during experiments, consistent with the estimate in literature [38], can explain the observed contribution of $\mathrm{O}_{3}$ etching for the typical ion flux and background pressure in our experiment. This comparison gives qualitative agreement between the experimental observations and calculated predictions. Sources of deviations at high energies can arise from either an under-estimate of the TRIDYN ballistic model to physical sputter yields of $\mathrm{O}_{2}^{+} / \mathrm{O}^{+}$on ruthenium, or a possible ion enhanced etching of ruthenium by ozone under simultaneous ion bombardment.

Mass analyzing and filtering the ion beam is therefore an important aspect for studying sputter yields by reactive ion species near the sputter threshold. While impurities (atomic ions and ozone) may be present in several percent in ion exposures for all materials studied, they do not significantly affect the physical sputter yields in most cases. However, the presence of a chemical etching component as demonstrated by ruthenium-ozone sputtering can greatly dominate physical sputter yields. Impurity (ozone) concentrations of even a few percent of the incident ion flux will create deviations of orders of magnitude and a physical sputter threshold cannot be reliably determined. Atomic $\mathrm{O}$ does not contribute to a chemical etching mechanism for $\mathrm{Ru}$ as no mass loss was observed in the experiments of metal target exposure to at-O.

\subsubsection{Chemical modifications: Oxygen ions vs. Atomic Oxy- gen}

The sputtering process also dynamically changes chemical composition of the target materials and usually a self-terminating oxide is formed due to a balance of sputtering and transport processes at steady state. In this section, we shall assess the oxidation states of the metal after ion irradiation.

In order to characterize the oxides formed at the end of the sputtering process as a function of ion energy, MSi samples exposed to $\mathrm{O}_{2}^{+} / \mathrm{O}^{+}$were analyzed by AR- 

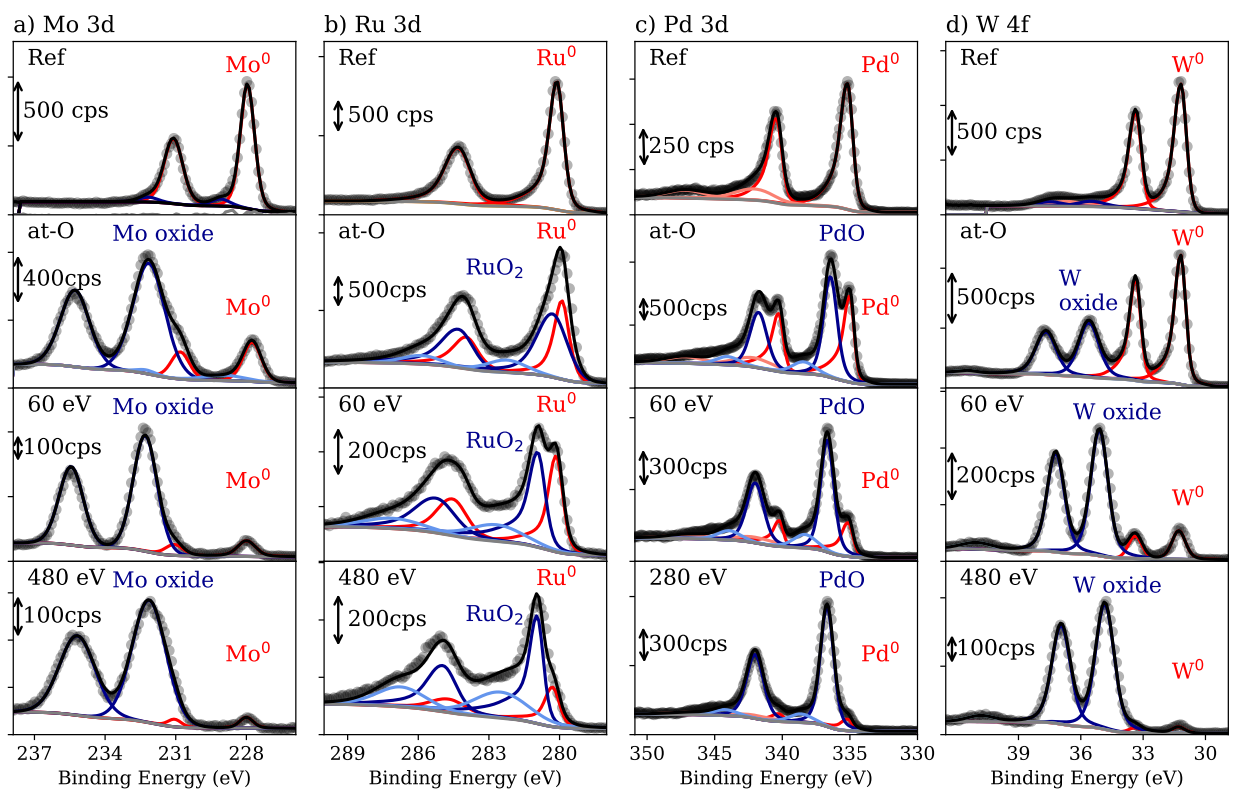

Figure 5.3: XPS spectra for a) molybdenum 3d b) ruthenium 3d c) palladium 3d and d) tungsten 4f core levels. Reference measurements are compared to oxygen ions of $60 \mathrm{eV}$ and $480 \mathrm{eV}$ as well as metal films exposed to atomic oxygen (at-O) at room temperature. Note, highest energy for palladium limited to $280 \mathrm{eV}$. Points represent measured data and lines denote the deconvolutions.

XPS according to Section 5.3.2. MSi samples were also exposed to at-O in order to compare the chemical states formed for similar oxygen species under two different oxidation mechanisms. On one hand, the kinetics for oxidation by oxygen ions are expected to be governed by the ion energy. Oxide formation occurs by athermal activation of chemical and diffusion pathways [41]. On the other hand, MSi samples exposed to atomic oxygen at room temperature are expected to oxidize following the Cabrera-Mott (CM) mechanism [20]. According to this theory, a strong electric field - generated by the oxygen and metal interaction - promotes positive metal ion diffusion towards the surface to react with the adsorbed oxygen, forming a new metal oxide layer at the outer surface. This electric field reduces in magnitude as the oxide thickness grows and a limiting oxide is formed when the electric field no longer reduces the diffusion barrier. Figure 5.3 shows a compendium of XPS measurements of $3 \mathrm{~d}$ levels of molybdenum, ruthenium and palladium and $4 \mathrm{f}$ levels of tungsten. Reference measurements with unexposed, as-deposited samples are shown along with spectra from the lowest and highest ion energy used in the experiments. Results from oxidation by atomic oxygen are also shown. Figure 5.4 additionally shows the O1s peaks for the samples in the same order.

Fits to XPS data were performed by first evaluating the as-deposited metal spectra to assess metal peak position and asymmetry. Oxide peaks in the metal core levels of oxygen exposed samples were then fit with a symmetric peak for molybdenum [42, 43] and tungsten [43] oxides and an asymmetric peak for ruthenium [44] 
and palladium [45]. The metal peak positions were fixed to the reference measurements for comparison of oxide components between ion and at-O exposed samples. Metals like molybdenum and tungsten exhibit multiple oxides within a small BE range. For simplicity, the oxide components were fit with a single peak while the full-width half maximum (FWHM) was allowed to vary. A larger FWHM was interpreted as a metal containing stoichiometric and non-stoichiometric states. The peak positions and FWHMs for the metal reference and various oxide samples are compiled in Table 5.3.

Magnetron sputter deposited films of molybdenum exhibit a Mo $3 \mathrm{~d}_{5 / 2}$ ground state peak at a binding energy (BE) of $228.0 \mathrm{eV}$ consistent with reports of metallic molybdenum [46]. Peaks at higher binding energy develop upon exposure of metallic films either to neutral at-O or $\mathrm{O}_{2}^{+} / \mathrm{O}^{+}$ions. Mo is known to form multiple stoichiometries [42], including upon oxygen ion bombardment at steady state [15] with $\mathrm{Mo}^{4+}(229 \mathrm{eV})$ being the most abundant at $1 \mathrm{keV} \mathrm{O}{ }_{2}^{+}$ion energies followed by $\mathrm{Mo}^{5+}(231.2 \mathrm{eV})$ and $\mathrm{Mo}^{6+}(232.4 \mathrm{eV})$. A comparison to our experimental ARXPS spectra rules out the formation of $\mathrm{Mo}^{4+}$ species during ion bombardment or at-O exposures. The $\mathrm{Mo}^{6+}$ oxidation state dominates in the MSi samples with $\mathrm{Mo}^{5+}$ making up a minority in the oxide at higher $\mathrm{O}_{2}^{+}$ion energies which is encompassed in the larger FWHM. An increase in FWHM would then, in our case, indicate the presence of sub-stoichiometric oxide of $\mathrm{Mo}^{5+}$ present at lower BE. The metal oxide peak positions of Mo $3 \mathrm{~d}_{5 / 2}$ were found to be invariant to the method of oxidation. This implies the most abundant stoichiometry (leading to the most intense XPS intensity) remains the same for all oxygen exposed films. The FWHMs indicate a more stoichiometric oxide formed at low ion energies which reduces as the FWHM broadens to include sub-stoichiometric oxides at high ion energy. The high ion energy oxide peaks are comparable to the peaks developed after at-O exposure. The O1s peaks positions and FWHMs do not vary with oxidation technique.

Ruthenium surfaces present quite slow oxidation at ambient conditions [47]. Upon exposure to at-O, the development of a sharp $\mathrm{RuO}_{2}$ peak does not occur due to the small separation of the peaks $(0.8 \mathrm{eV})$ conflated by the shallow depth of oxidation. A broadening of the Ru3d peak along with a clear O1s peak however indicate the formation of an oxide. For at-O exposed samples, a binding energy of $280.6 \mathrm{eV}$ is assigned to the $\mathrm{Ru}_{3} \mathrm{~d}_{5 / 2}$ peak, based on the fact that at more surface sensitive angles, this $\mathrm{RuO}_{2}$ peak is distinct from its metallic counterpart. Energetic $\mathrm{O}_{2}^{+} / \mathrm{O}^{+}$shifts the oxide binding energy by as much as $0.6 \mathrm{eV}$ towards higher binding energies. The $\mathrm{O} 1 \mathrm{~s}$ peak varies between the two methods by $0.2 \mathrm{eV}$, but more importantly, the $\mathrm{O} 1 \mathrm{~s}$ peak for $\mathrm{O}_{2}^{+} / \mathrm{O}^{+}$ion exposures is much sharper relating to a more ordered and well defined oxide. The additional component in the O1s region arising from atmospheric exposure and adsorption of water or formation of hydroxides is difficult to constrain for the two oxidation techniques. This is in part to slight variations in atmospheric exposures and varying surface structures which could not be measured in the present experiments.

Palladium is inert to oxidation under ambient conditions. Interactions with highly reactive and energetic species however, lead to formation of an oxide with the most stable and consequently most studied being $\mathrm{PdO}[45,48,49]$. No difference in peak positions and valency were observed for palladium for either kinds of oxidizing 

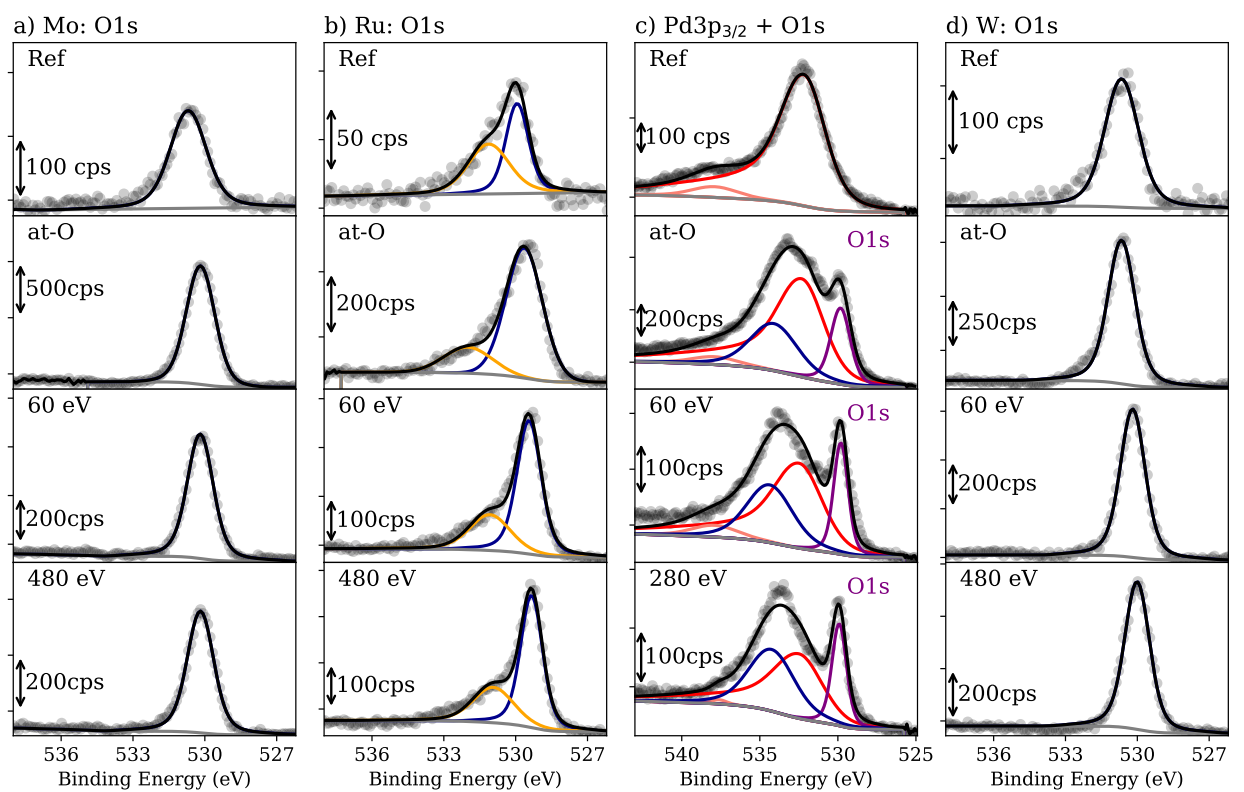

Figure 5.4: O1s spectra of a) molybdenum, b) ruthenium, c) palladium and d) tungsten plotted for reference, at-O and oxygen ion exposed samples, with indicated ion energy. Palladium $3 \mathrm{p}_{3 / 2}$ peaks are also fitted due to their overlap with O1s. Note the highest energy for Pd is $280 \mathrm{eV}$.

species. The $\mathrm{Pd} 3 \mathrm{~d}_{5 / 2}$ peak positions are consistent with $\mathrm{PdO}$ [45] with $\mathrm{Pd} 3 \mathrm{~d}_{5 / 2}$ and $\mathrm{Pd} 3 \mathrm{~d}_{3 / 2}$ present at $336.6 \pm 0.1 \mathrm{eV}$ and $341.9 \pm 0.1 \mathrm{eV}$ respectively.

Tungsten behaves similar to molybdenum in that it contains multiple oxidation states within a BE region of 3-5 eV. The metal peak for unexposed tungsten is consistent with literature at a binding energy of $31.2 \mathrm{eV} \mathrm{[46].} \mathrm{We} \mathrm{fit} \mathrm{the} \mathrm{entirety}$ of the oxide signal with a single peak while varying the FWHM. Reports on 1 $\mathrm{keV}_{2}^{+}$ion bombardment [15] of tungsten shows formation of $\mathrm{W}^{4+}(32.7 \mathrm{eV})$, $\mathrm{W}^{5+}(34.4 \mathrm{eV})$ and $\mathrm{W}^{6+}(35.6 \mathrm{eV})$ oxide species at steady state. Upon oxidation with $60 \mathrm{eV}$ ions, a $\mathrm{W}_{4 / 2}$ peak develops at a $\mathrm{BE}$ of $35.1 \mathrm{eV}$. The $\mathrm{BE}$ moves to lower values (lower oxidation states) of $34.8 \mathrm{eV}$ at an ion energy of $480 \mathrm{eV}$. Ion exposed $\mathrm{W}$ thus forms $\mathrm{W}^{5+}$ with small quantities of $\mathrm{W}^{6+}$ oxidation states at low ion energies. With increasing energy, the abundance of $\mathrm{W}^{5+}$ increases and the oxide formed is sub-stoichiometric. At-O oxidation leads to a much higher BE peak at $35.6 \mathrm{eV}$ corresponding to $\mathrm{W}^{6+}$. The energetics involved in ion bombardment leads to preferential sputtering of oxygen from the oxide, thus generating a lower stoichiometry. The O1s peak also shifts to higher B.E. by $0.4-0.6 \mathrm{eV}$ when comparing ion irradiation to at-O exposure.

Thus, we find that every target studied undergoes oxidation with the oxidation generally being similar for oxygen ions and atomic oxygen. The marked difference between them is the intensity of the elemental (ground state) peak, meaning the depth of oxygen transport being more shallow for at-O. In the following section, we shall assess the depth of oxygen transport by employing the AR-XPS results. 
Table 5.3: Peak BEs (in eV) for molybdenum, ruthenium, palladium and tungsten samples as measured by XPS. The full width at half maximum (FWHM) of each peak is supplied in parentheses. For ion exposed and at-O exposed samples, the metallic peaks were fixed according to the as-deposited reference measurement.

\begin{tabular}{|c|c|c|c|c|c|}
\hline & \multicolumn{5}{|c|}{ Orbital Binding Energies $(\mathrm{eV})$} \\
\hline & $3 \mathrm{~d}_{5 / 2}$ & $3 \mathrm{~d}_{3 / 2}$ & $\mathrm{O} 1 \mathrm{~s}$ & $4 f_{7 / 2}$ & $4 f_{5 / 2}$ \\
\hline Mo (as deposited) & $228.0(0.7)$ & $231.1(0.9)$ & $530.7(1.8)$ & & \\
\hline Mo oxide (at-O) & $232.2(1.5)$ & $235.3(1.5)$ & $530.3(1.3)$ & & \\
\hline Mo oxide $(60 \mathrm{eV})$ & $232.3(1.2)$ & $235.5(1.2)$ & $530.2(1.4)$ & & \\
\hline Mo oxide $(480 \mathrm{eV})$ & $232.2(1.7)$ & $235.2(1.8)$ & $530.2(1.3)$ & & \\
\hline $\mathrm{Ru}$ (as deposited) & $280.1(0.8)$ & $284.3(1.3)$ & $529.9(1.1)$ & & \\
\hline Ru oxide (at-O) & $280.6(1.1)$ & $284.6(1.2)$ & $529.6(1.8)$ & & \\
\hline $\mathrm{Ru}$ oxide $(60 \mathrm{eV})$ & $280.9(0.9)$ & $285.2(2.1)$ & $529.4(1.2)$ & & \\
\hline $\mathrm{Ru}$ oxide $(480 \mathrm{eV})$ & $280.9(0.8)$ & $285.1(1.5)$ & $529.3(1.1)$ & & \\
\hline $\mathrm{Pd}$ (as deposited) & $335.1(1.1)$ & $340.4(1.3)$ & - & & \\
\hline Pd oxide (at-O) & $336.5(1.2)$ & $341.8(1.5)$ & $529.8(1.5)$ & & \\
\hline Pd oxide $(60 \mathrm{eV})$ & $336.7(1.2)$ & $342.0(1.5)$ & $529.8(1.1)$ & & \\
\hline Pd oxide $(280 \mathrm{eV})$ & $336.1(1.2)$ & $342.0(1.5)$ & $529.9(1.1)$ & & \\
\hline $\mathrm{W}$ (as deposited) & & & $530.6(1.7)$ & $31.2(0.7)$ & $33.3(0.7)$ \\
\hline $\mathrm{W}$ oxide $($ at- $\mathrm{O})$ & & & $530.6(1.4)$ & $35.6(1.2)$ & $37.7(1.2)$ \\
\hline $\mathrm{W}$ oxide $(60 \mathrm{eV})$ & & & $530.2(1.2)$ & $35.1(1.0)$ & $37.2(1.0)$ \\
\hline W oxide $(480 \mathrm{eV})$ & & & $530.0(1.2)$ & $34.8(1.1)$ & $36.9(1.1)$ \\
\hline
\end{tabular}

\subsubsection{Oxide Thickness}

The XPS spectra depict a notable difference between the oxidizing species: at-O exposures retain a significant portion of the metal peak intensity from metal in the elemental state signifying a shallow oxidation. The diminishing elemental metal peaks with increasing ion energy indicate a deeper penetration of the oxygen due to ballistic impact. To estimate the depth of oxides formed, thicknesses of the oxide were determined using the multi-overlayer model described in section 5.3.2.

Figure 5.5 shows the experimentally determined oxide thicknesses from ARXPS measurements and depth of penetration of ions predicted by TRIDYN. The depth of the oxide/metal interface in the simulated profiles was determined where the $\mathrm{O}$ concentration drops to $0.5 \times O_{\text {sat }}$. The multi-overlayer model relies on the location of the interface of an overlayer to determine thicknesses. The approximation made to assess the interface of the profiles generated by TRIDYN can then be directly compared to the experimental results. TRIDYN simulations were carried out assuming an oxygen atomic fraction up to saturation of a stoichiometric oxide and bulk oxide density. The ion transport mean free path is inversely proportional to the atomic density of the target material. For the purely implantation driven simulations (without SBE changes) that consider ion transport and a stoichiometric oxide formation (near bulk oxide density), the simulations present the maximum 

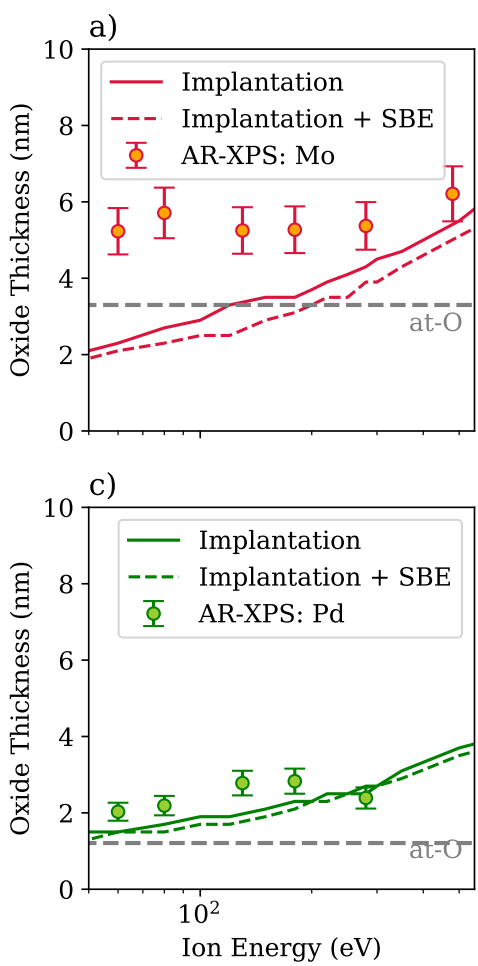

b)

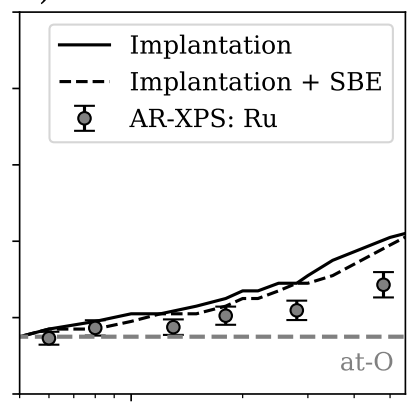

d)

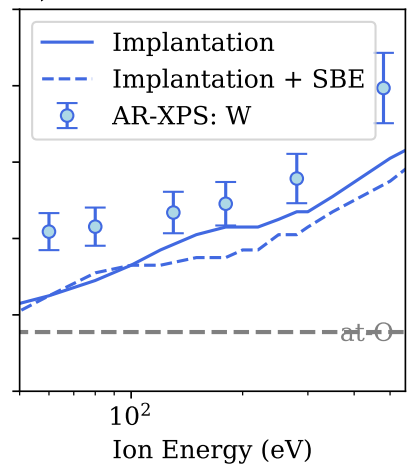

Figure 5.5: Experimental oxide thicknesses calculated using equation 5.2 after deconvolution of AR-XPS peaks for a) molybdenum b) ruthenium c) palladium and d) tungsten. Implantation depth from TRIDYN simulations are shown for a pure implantation case (solid line) and an implantation with a surface binding energy change (dashed). Experimental thickness was calculated using bulk metal oxide densities. The thickness obtained from at-O exposures (gray dashed line) is also plotted for comparison.

oxide thickness that can be formed due to ballistic collisions only. The results in Figure 5.5 show ballistic transport correctly predicts the oxide thickness only for $\mathrm{Ru}$ and $\mathrm{Pd}$ while Mo and $\mathrm{W}$ show significant deviations.

The deviations signify the presence of diffusional components, possibly natural or enhanced by the ion irradiation process. We shall consider this possibility in the following section.

\section{Oxygen Transport: Radiation Enhanced Diffusion}

Ion implantation processes can usually be described as a competition between sputtering and implantation where a higher energy leads to a higher penetration range but also increased sputtering, forming a limited implantation thickness. Relocations can further enhance the depth to which ion species can be transported. At high energies $(>1 \mathrm{keV})$, this is generally the case, however, for certain material combinations, effects of diffusion of implanted atoms cannot be ignored. This was demonstrated by Vancauwenberge [50] and Todorov [51] for oxidation of silicon by 
low energy oxygen ions. We can describe the transport of oxygen into the metal as a partial differential equation (PDE) of the form:

$$
\frac{\partial O(x)}{\partial t}=\frac{\partial}{\partial x}\left(D_{0} \frac{\partial}{\partial x} O(x)\right)+S(x)-Z(x)+g(x)
$$

where $O(x)$ represents the concentration of oxygen, $S(x)$ is the deposition function, which is correlated to the incoming ion flux $\left(j_{0}\right)$ and the reflection coefficient $(R)$ dependent on incident energy [52]. In principle, $S$ is a representation of the four moments of ion incorporation: range, straggle, skewness and kurtosis [52, 53]. $Z(x)$ is the etching function for removal of oxygen from the target. $g(x)$ is a generation term bringing in interstitials into a slab $\Delta x$ in the target. The diffusion coefficient is described as:

$$
D_{0}=D_{\text {therm }}+D_{\text {rad }}\left(\frac{n_{O}}{n_{\text {total }}^{\max }}\right)
$$

where $D_{\text {therm }}$ is the thermal diffusivity $\left(\mathrm{cm}^{2} / \mathrm{s}\right)$ of oxygen which scales with the concentration of oxygen in a lamella to account for different diffusion coefficients in the metal and the oxide. Here we use the oxygen diffusivity in the metal only as data on oxide diffusion for current transition metals is scarce. $D_{\text {rad }}$ is the radiation enhanced diffusivity which is limited to the region of ballistic collision induced damage and proportional to the concentration $n_{O}$, of oxygen atoms that are displaced by impacting ions. $n_{\text {total }}^{\max }$ is a normalization factor representing the total concentration of vacancies/interstitials generated. Here, impact onto an oxide can generate oxygen from the target, where the oxide is considered to be decomposed into free oxygen and the metal. The oxygen is then available to diffuse either further into the target, or outwards to the surface where it is lost. Beyond the damaged region, $D_{\text {rad }}$ is 0 . In the absence of any type of diffusion, the oxygen profile generated at each depth interval is determined by the deposition and etching components only and the results would match TRIDYN outputs.

TRIDYN already accounts for $S(x), Z(x)$ and $g(x)$ on the right hand side of equation 5.4. In order to address the diffusion of oxygen, an algorithm is used as follows: TRIDYN outputs a number of $\mathrm{O}$ concentration depth profiles and damage profiles corresponding to a partial fluence for a certain time step. After each time step, the concentration profile is allowed to diffuse for a time corresponding to the ratio of partial fluence to experimental flux $(\sim 87 \mathrm{~s})$. The resulting profile serves as initial condition for the next partial fluence as simulated by TRIDYN. The damage profile generated at a previous time step is used to assign a scaling for $D_{\text {rad }}$ under the assumption that the diffusion does not alter the damage profile in the target for the next time step. Any excess oxygen resulting from addition of partial fluences to the system, leading to over-stoichiometry is assumed to out-diffuse and a stoichiometric oxide is maintained. The PDE is discretized by a finite difference scheme as a central difference in space and a forward difference in time [54]. Integration is performed numerically and in order to fulfill the stability criterion for the PDE, smaller time steps were used such that $\Delta t<\Delta x^{2} / D_{0}$ where $\Delta t$ is the time step and $\Delta x$ is the spatial step $(0.2 \mathrm{~nm})$. This implementation is necessary as the built-in option in 
TRIDYN to simulate diffusive transport of oxygen results in unrealistically large oxygen penetration depths and lacks tunability.

We perform the above procedure for metals that show the largest discrepancy in oxide thickness from TRIDYN predictions: molybdenum and tungsten. The resulting oxide thicknesses are plotted in Figure 5.6. The thermal diffusivity of oxygen in tungsten has a value of $4.3 \times 10^{-19} \mathrm{~cm}^{2} / \mathrm{s}$ (at $380 \mathrm{~K}$ ) from [55]. A criterion for the choice of magnitude for $D_{\text {rad }}$ is that a single value (and not a functional form) should be able to describe the observations over a wide range of incident energies $[41,50]$. A $D_{\text {rad }}$ of $4.8 \times 10^{-16} \mathrm{~cm}^{2} / \mathrm{s}$ provides the best match to the experimental results which is $\sim 1000 \times$ greater than the thermal diffusivity.

The nature of $D_{\text {therm }}$ deserves some comment as previous reports $[50,56]$ describe it as a thermal diffusivity. $D_{\text {therm }}$ would then be influenced by temperature, as well as structure of the film unless $D_{\text {therm }}$ is a bulk diffusivity. For an a-thermal process such as ion beam oxidation, the ion energies cannot be directly translated to a macroscopic temperature to scale the diffusion coefficient given an Arrhenius form. The choice of temperature (here $380 \mathrm{~K}$ ) can influence $D_{\text {therm }}$ and affect the choice of $D_{\text {rad }}$. We attempt to remedy this by performing a crude sensitivity analysis on $D_{\text {therm }}$ which provides bounds for regions where $D_{\text {rad }}$ and $D_{\text {therm }}$ exist. The sensitivity of $D_{\text {rad }}$ to changes in $D_{\text {therm }}$ can be understood by varying $D_{\text {therm }}$ to $3.2 \times 10^{-18} \mathrm{~cm}^{2} / \mathrm{s}$ (corresponding to $400 \mathrm{~K}$ ). A value of $D_{\text {rad }}$ of $\sim 2.2 \times 10^{-17} \mathrm{~cm}^{2} / \mathrm{s}$ provides a reasonable agreement with the oxide thickness which is over-estimated (by $20 \%$ of experimental value) at $60 \mathrm{eV}$ and under-estimated (by $5 \%$ ) for $500 \mathrm{eV}$.

We posit that $D_{\text {therm }}$ in the case of metals exposed to oxygen ions could be functionally dependent on the field produced between the free oxygen created by ion bombardment at the interface of the metal oxide and the metal underneath. The oxidation process would then be driven in a CM-like mechanism [57]. This interpretation of $D_{\text {therm }}$ does not directly negate the analysis presented so far. It does imply that the assignment of a particular temperature is erroneous, however, the magnitude of diffusivity is necessary for oxygen transport and can stem from a CM-like mechanism. Assigning a diffusivity to a CM driven oxide for comparison to present values is not trivial and requires further study.

$D_{\text {therm }}$ values for Mo were not readily available, however, assuming it to be in the same order of magnitude as $\mathrm{W}$, the oxide thickness is predicted with $D_{\text {therm }}$ $=4 \times 10^{-18} \mathrm{~cm}^{2} / \mathrm{s}$ and $D_{\text {rad }}=4.8 \times 10^{-18} \mathrm{~cm}^{2} / \mathrm{s}$. The comparable magnitudes of both diffusivities lead to a near-constant 'saturable' thickness as a function of energy. According to Vancauwenberghe [50], we find that both $D_{\text {rad }}$ and $D_{\text {therm }}$ are responsible for diffusion, with $D_{\text {rad }}$ limited to the region where ions generate damage (vacancies / interstitials), and $D_{\text {therm }}$ pervasive throughout the target. The surface acts as a sink to outward diffusing oxygen and prevents $D_{\text {therm }}$ from continually diffusing oxygen deeper into the target. In both cases, for molybdenum and tungsten, a single value of $D_{\text {rad }}$ explains the results, over a wide range of energies.

Ruthenium and palladium show oxide thicknesses that are well predicted by TRIDYN simulations. In the case of ruthenium, the anomalous sputtering of the metal can be posited to reduce deeper oxygen transport. $D_{0}$ may still play a role in ruthenium oxidation by ion bombardment, which will require elimination of the species involved in chemical sputtering. Sputter yields as well as the depth of oxide 
a) Mo

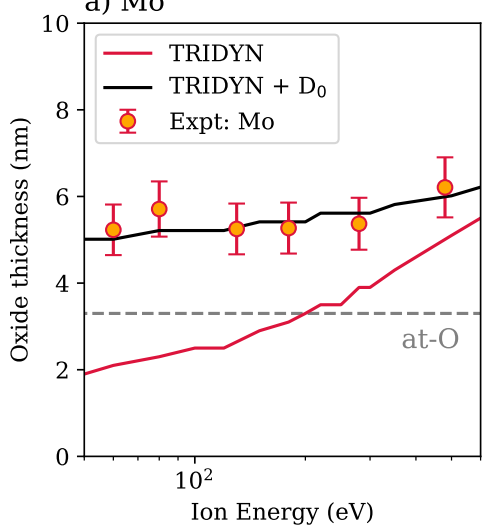

b) $\mathrm{W}$

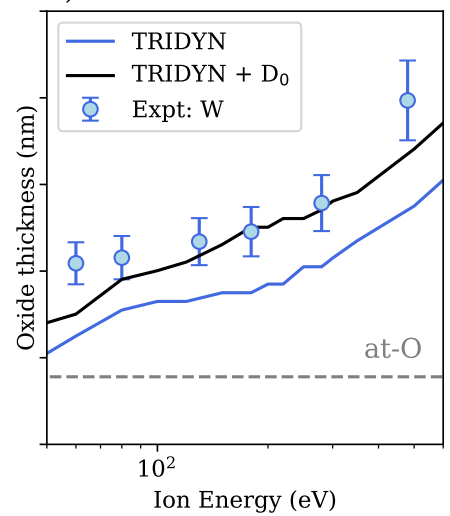

Figure 5.6: Experimental oxide thickness from AR-XPS measurements for a) molybdenum and b) tungsten as shown in Figure 5.5. Also included are the TRIDYN simulations for the pure implantation case which account for implantation, sputtering and interstitial formation. The solution for the PDE in equation 5.4 shows that an additional radiation enhanced diffusion better describes the transport processes (black line). Dashed lines denote the thickness of the oxide by

penetration in palladium are well described by ballistic transport without having to account for additional oxygen diffusivity. Thermal diffusivity of oxygen in palladium is low [58] with oxide penetration limited to $0.5 \mathrm{ML}$. For $D_{\text {therm }}$ in palladium to be comparable to that of tungsten and molybdenum, requires temperatures $>$ $1000 \mathrm{~K}$ and pressures in the order of $10^{3}-10^{4} \mathrm{~Pa}$ [59]. It can be posited that a $D_{\text {rad }}$ component can exist in palladium, however, in cases where $D_{\text {rad }}$ is the primary driver, the transport of oxygen is not significantly greater than the prediction by TRIDYN and similar models [50]. This would explain the agreement between experiment and TRIDYN simulations without $D_{0}$.

Oxygen ion exposures, studied in this work, can be compared to our recent report on transport and retention of nitrogen in the same metals upon nitrogen ion incidence [60]. It was implicitly shown in [60] that nitrogen incorporation is mediated by ballistic processes. The nitrogen content in the target materials can be accounted by TRIDYN without influence of additional diffusion processes. This is in contrast to our studies of oxygen where diffusion clearly plays a role. We hypothesize the reason for such behavior to be due to longer (shorter) diffusional time scales of nitrogen (oxygen) within the nitride (oxide) formed at steady state. In the case of tungsten, for instance, time scales for nitrogen diffusion in the order of $1000 \mathrm{~s}$ were observed at elevated temperatures $(970 \mathrm{~K})$ [61]. In contrast, the time sales for oxygen transport in tungsten, from this work can be obtained from a consideration: $\Delta t \sim \Delta x^{2} / D_{0}$, using $\Delta x=0.2 \mathrm{~nm}$ as the jump distance in tungsten [62], lie between $\sim 9 \mathrm{~ms}-9 \mathrm{~s}$. The exact nature of diffusional processes within the two ion exposed samples warrants investigation as one is a desorptive process directed outwards, and the other is directed inwards. 


\subsection{Conclusions}

In this work, we have reported the interaction of low energy oxygen ions with transition metals relevant for semiconductor, photo-lithography and fusion applications in the energy range of $50-500 \mathrm{eV}$. Ballistic damage was studied by measuring sputter yields for molybdenum, ruthenium, palladium and tungsten near the sputter threshold. Comparison of experiments with TRIDYN simulations showed agreement for palladium and deviations for the rest of the metals studied. Factors such as volatile compound formation and molecular ion effects were discussed and found to affect ruthenium significantly. Ozone ( $<1 \%$ of background gas) produced in the plasma is hypothesized to cause chemical etching of ruthenium. Molybdenum and tungsten are also potentially affected by volatile oxide formation, but the precise nature of the interaction is yet unknown. All metals form oxides which are not necessarily governed by ballistic transport only. Thermal and radiation enhanced diffusion of oxygen was hypothesized for oxygen transport, where ion damage creates free oxygen which diffuses further into the bulk. A diffusion algorithm was implemented using TRIDYN simulations of depth profiles in order to assess the effect of radiation enhanced diffusion on oxygen transport. Transport of oxygen within the film (via oxide thickness measurements) was found to be governed by ballistic collisions for palladium and ruthenium. Molybdenum and tungsten exhibited oxygen transport depth which is under-estimated by ballistic models and was explained by radiation enhanced diffusion. Ruthenium can be susceptible to radiation enhanced diffusion of oxygen, however, due to the high sputter rate (from ozone etching), a conclusion could not be made. 


\section{References}

[1] S. Fukatsu, Y. Ishikawa, T. Saito, and N. Shibata, Sige-based semiconductoron-insulator substrate created by low-energy separation-by-implanted-oxygen, Applied Physics Letters 72, 3485 (1998).

[2] R. Coloma Ribera, R. W. E. van de Kruijs, J. M. Sturm, A. E. Yakshin, and F. Bijkerk, Intermixing and thermal oxidation of zro2 thin films grown on a-si, sin, and sio2 by metallic and oxidic mode magnetron sputtering, Journal of Applied Physics 121, 115303 (2017).

[3] D. Mutschall, K. Holzner, and E. Obermeier, Sputtered molybdenum oxide thin films for nh3 detection, Sensors and Actuators B: Chemical 36, 320 (1996), proceedings of the Sixth International Meeting on Chemical Sensors.

[4] Y. Hirooka, M. Bourham, J. Brooks, R. Causey, G. Chevalier, R. Conn, W. Eddy, J. Gilligan, M. Khandagle, and Y. Ra, Evaluation of tungsten as a plasma-facing material for steady state magnetic fusion devices, Journal of Nuclear Materials 196-198, 149 (1992), plasma-Surface Interactions in Controlled Fusion Devices.

[5] M. H. L. Van Der Velden, W. J. M. Brok, J. J. A. M. Van Der Mullen, W. J. Goedheer, and V. Banine, Particle-in-cell Monte Carlo simulations of an extreme ultraviolet radiation driven plasma, Physical Review E - Statistical, Nonlinear, and Soft Matter Physics 73, 1 (2006).

[6] P. Phadke, J. M. Sturm, R. W. van de Kruijs, and F. Bijkerk, Near-threshold sputter yields of ruthenium under argon and nitrogen ion bombardment, Nuclear Instruments and Methods in Physics Research, Section B: Beam Interactions with Materials and Atoms 458, 169 (2019).

[7] P. Stott, C. Daughney, and R. Ellis, Control of re-cycling and impurities in the ATC tokamak by means of gettered surfaces, Nuclear Fusion 15, 431 (1975).

[8] N. Matsunami, N. Ohno, and M. Tokitani, Deuterium retention in tungsten oxide under low energy d2+ plasma exposure, Journal of Nuclear Materials 390-391, 693 (2009), proceedings of the 18th International Conference on Plasma-Surface Interactions in Controlled Fusion Device.

[9] V. K. Alimov, Deuterium retention in pure and mixed plasma facing materials, Physica Scripta T108, 46 (2004).

[10] P. He, Z. Zhang, W. Xia, L. Shu, X. Ma, F. Gou, and K. Zhang, Compatibility between high-flux helium plasma irradiated molybdenum and liquid lithium, Journal of Nuclear Materials 509, 736 (2018).

[11] D. L. Rudakov, J. A. Boedo, R. A. Moyer, A. Litnovsky, V. Philipps, P. Wienhold, S. L. Allen, M. E. Fenstermacher, M. Groth, C. J. Lasnier, R. L. Boivin, N. H. Brooks, A. W. Leonard, W. P. West, C. P. C. Wong, A. G. McLean, P. C. Stangeby, G. De Temmerman, W. R. Wampler, and J. G. Watkins, First 
tests of molybdenum mirrors for iter diagnostics in diii-d divertor, Review of Scientific Instruments 77, 10F126 (2006).

[12] Y. Yao, Z. Hargitai, M. Albert, R. Albridge, a. Barnes, J. Gilligan, B. Pratt Ferguson, G. Lüpke, V. Gordon, N. Tolk, J. Tully, G. Betz, and W. Husinsky, New Molecular Collisional Interaction Effect in Low-Energy Sputtering, Physical Review Letters 81, 550 (1998).

[13] E. Hechtl and J. Bohdansky, Sputtering behavior of graphite and molybdenum at low bombarding energies, Journal of Nuclear Materials 123, 1431 (1984).

[14] E. Hechtl, J. Bohdansky, and J. Roth, The sputtering yield of typical impurity ions for different fusion reactor materials, Journal of Nuclear Materials 103, 333 (1981).

[15] N. V. Alov, Surface oxidation of metals by oxygen ion bombardment, Nuclear Instruments and Methods in Physics Research Section B: Beam Interactions with Materials and Atoms 256, 337 (2007), atomic Collisions in Solids.

[16] I. Saric, R. Peter, I. Kavre, I. J. Badovinac, and M. Petravic, Oxidation of nickel surfaces by low energy ion bombardment, Nuclear Instruments and Methods in Physics Research Section B: Beam Interactions with Materials and Atoms 371, 286 (2016), the 22nd International Conference on Ion Beam Analysis (IBA 2015).

[17] R. Peter, I. Saric, I. K. Piltaver, I. J. Badovinac, and M. Petravic, Oxide formation on chromium metal surfaces by low-energy oxygen implantation at room temperature, Thin Solid Films 636, 225 (2017).

[18] S. M. Wu, R. W. E. van de Kruijs, E. Zoethout, and F. Bijkerk, Sputtering yields of Ru, Mo, and Si under low energy Ar+ bombardment, Journal of Applied Physics 106, 054902 (2009).

[19] B. Nichols and F. C. Witteborn, Measurements of resonant charge exchange cross sections in nitrogen and argon between 0.5 and $17 \mathrm{ev}$, Nasa Technical Note , 1 (1966).

[20] N. Cabrera and N. F. Mott, Theory of the oxidation of metals, Reports on Progress in Physics 12, 163 (1949).

[21] C. R. Stilhano Vilas Boas, J. M. Sturm, and F. Bijkerk, Oxidation of metal thin films by atomic oxygen: A low energy ion scattering study, Journal of Applied Physics 126, 155301 (2019).

[22] S. Ramanathan, D. Chi, P. C. McIntyre, C. J. Wetteland, and J. R. Tesmer, Ultraviolet-ozone oxidation of metal films, Journal of The Electrochemical Society 150, F110 (2003).

[23] B. Herd and H. Over, Atomic scale insights into the initial oxidation of ru(0001) using atomic oxygen, Surface Science 622, 24 (2014). 
[24] W. Möller, W. Eckstein, and J. P. Biersack, Tridyn-binary collision simulation of atomic collisions and dynamic composition changes in solids, Computer Physics Communications 51, 355 (1988).

[25] W. Möller and D. Güttler, Modeling of plasma-target interaction during reactive magnetron sputtering of TiN, Journal of Applied Physics 102 (2007), $10.1063 / 1.2800262$.

[26] Z. Y. Chen, A. Bogaerts, D. Depla, and V. Ignatova, Dynamic Monte Carlo simulation for reactive sputtering of aluminium, Nuclear Instruments and Methods in Physics Research, Section B: Beam Interactions with Materials and Atoms 207, 415 (2003).

[27] K. Dobes, V. Smejkal, T. Schäfer, and F. Aumayr, Interaction between seeding gas ions and nitrogen saturated tungsten surfaces, International Journal of Mass Spectrometry 365-366, 64 (2014).

[28] S. Parascandola, T. Telbizova, O. Kruse, and W. Möller, The influence of the oxygen partial pressure on the ion nitriding of aluminium - an investigation by means of real time elastic recoil detection analysis, Nuclear Instruments and Methods in Physics Research Section B: Beam Interactions with Materials and Atoms 161-163, 406 (2000).

[29] P. J. Cumpson, The thickogram: a method for easy film thickness measurement in xps, Surface and Interface Analysis 29, 403 (2000).

[30] P. J. Cumpson and M. P. Seah, Elastic scattering corrections in aes and xps. ii. estimating attenuation lengths and conditions required for their valid use in overlayer/substrate experiments, Surface and Interface Analysis 25, 430 (1997).

[31] A. Dolgov, D. Lopaev, T. Rachimova, A. Kovalev, A. Vasil'eva, C. J. Lee, V. M. Krivtsun, O. Yakushev, and F. Bijkerk, Comparison of h2and he carbon cleaning mechanisms in extreme ultraviolet induced and surface wave discharge plasmas, Journal of Physics D: Applied Physics 47, 065205 (2014).

[32] M. Saidoh, H. Gnaser, and W. O. Hofer, On the chemical sputtering of oxygenexposed molybdenum, Applied Physics A Solids and Surfaces 40, 197 (1986).

[33] T. Saburi, H. Murata, T. Suzuki, Y. Fujii, and K. Kiuchi, Oxygen plasma interactions with molybdenum: Formation of volatile molybdenum oxides, Journal of Plasma and Fusion Research 78, 3 (2002).

[34] M. Saidoh, Sputter erosion of molybdenum and tungsten due to ion bombardment at temperatures up to $1500^{\circ} \mathrm{c}$, Journal of Nuclear Materials 128-129, 540 (1984).

[35] M. Nakahara, S. Tsunekawa, K. Watanabe, T. Arai, T. Yunogami, and K. Kuroki, Etching technique for ruthenium with a high etch rate and high selectivity using ozone gas, Journal of Vacuum Science \& Technology B: Microelectronics and Nanometer Structures Processing, Measurement, and Phenomena 19, 2133 (2001). 
[36] J. Kitayama and M. Kuzumoto, Theoretical and experimental study on ozone generation characteristics of an oxygen-fed ozone generator in silent discharge, Journal of Physics D: Applied Physics 30, 2453 (1997).

[37] F. Pontiga, C. Soria, A. Castellanos, and J. D. Skalny, A study of ozone generation by negative corona discharge through different plasma chemistry models, Ozone: Science \& Engineering 24, 447 (2002), https://doi.org/10.1080/01919510208901634 .

[38] M. Zeuner, J. Meichsner, H. Neumann, F. Scholze, and F. Bigl, Design of ion energy distributions by a broad beam ion source, Journal of Applied Physics 80, 611 (1996).

[39] R. Hashaikeh, I. Butler, and J. Kozinski, Thin-film ruthenium dioxide coatings via ozone-mediated chemical vapor deposition, Thin Solid Films 515, 1918 (2006).

[40] C. C. Hsu, J. W. Coburn, and D. B. Graves, Etching of ruthenium coatings in o2- and cl2-containing plasmas, Journal of Vacuum Science \& Technology A 24, 1 (2006).

[41] J. W. Rabalais, Low energy ion-surface interactions, Vol. 2 (Wiley, 1994).

[42] J. Baltrusaitis, B. Mendoza-Sanchez, V. Fernandez, R. Veenstra, N. Dukstiene, A. Roberts, and N. Fairley, Generalized molybdenum oxide surface chemical state xps determination via informed amorphous sample model, Applied Surface Science 326, 151 (2015).

[43] N. V. Alov, Determination of the states of oxidation of metals in thin oxide films by $x$-ray photoelectron spectroscopy, Journal of Analytical Chemistry 60, 431 (2005).

[44] D. J. Morgan, Resolving ruthenium: Xps studies of common ruthenium materials, Surface and Interface Analysis 47, 1072 (2015).

[45] M. Brun, A. Berthet, and J. Bertolini, Xps, aes and auger parameter of pd and pdo, Journal of Electron Spectroscopy and Related Phenomena 104, 55 (1999).

[46] I. Takano, S. Isobe, T. Sasaki, and Y. Baba, Nitrogenation of various transition metals by n+2-ion implantation, Applied Surface Science 37, 25 (1989).

[47] J. Hrbek, D. G. van Campen, and I. J. Malik, The early stages of ruthenium oxidation, Journal of Vacuum Science \& Technology A 13, 1409 (1995).

[48] M. C. Militello and S. J. Simko, Palladium oxide (pdo) by xps, Surface Science Spectra 3, 395 (1994).

[49] M. Peuckert, Xps study on surface and bulk palladium oxide, its thermal stability, and a comparison with other noble metal oxides, The Journal of Physical Chemistry 89, 2481 (1985). 
[50] O. Vancauwenberghe, N. Herbots, and O. C. Hellman, Role of ion energy in ion beam oxidation of semiconductors: Experimental study and model, Journal of Vacuum Science \& Technology A 10, 713 (1992).

[51] S. S. Todorov and E. R. Fossum, Growth mechanism of thin oxide films under low energy oxygen ion bombardment, Journal of Vacuum Science \& Technology B: Microelectronics Processing and Phenomena 6, 466 (1988).

[52] W. Möller, F. Besenbacher, and J. Bottiger, Saturation and isotope mixing during low-temperature implantations of hydrogen into metals, Applied Physics A Solids and Surfaces 27, 19 (1982).

[53] W. Eckstein, Computer simulation of ion-solid interactions, Vol. 10 (Springer Science \& Business Media, 2013).

[54] M. H. Holmes, Introduction to Numerical Methods Differential Equations (Springer Science, New York, 2007).

[55] V. Sikka and C. Rosa, The oxidation kinetics of tungsten and the determination of oxygen diffusion coefficient in tungsten trioxide, Corrosion Science 20, 1201 (1980).

[56] P. J. Doyle, K. M. Benensky, and S. J. Zinkle, Modeling the impact of radiationenhanced diffusion on implanted ion profiles, Journal of Nuclear Materials 509, 168 (2018).

[57] S. Gurbán, P. Petrik, M. Serényi, A. Sulyok, M. Menyhárd, E. Baradács, B. Parditka, C. Cserháti, G. Langer, and Z. Erdélyi, Electron irradiation induced amorphous sio 2 formation at metal oxide/si interface at room temperature; electron beam writing on interfaces, Scientific reports 8, 2124 (2018).

[58] E. Voogt, A. Mens, O. Gijzeman, and J. Geus, Adsorption of oxygen and surface oxide formation on pd(111) and pd foil studied with ellipsometry, leed, aes and xps, Surface Science 373, 210 (1997).

[59] J. Gegner, G. Hörz, and R. Kirchheim, Diffusivity and solubility of oxygen in solid palladium, Journal of Materials Science 44, 2198 (2009).

[60] P. Phadke, J. M. Sturm, R. W. van de Kruijs, and F. Bijkerk, Sputtering and nitridation of transition metal surfaces under low energy, steady state nitrogen ion bombardment, Applied Surface Science, 144529 (2019).

[61] G. Meisl, K. Schmid, O. Encke, T. Höschen, L. Gao, and C. Linsmeier, Implantation and erosion of nitrogen in tungsten, New Journal of Physics 16 (2014), 10.1088/1367-2630/16/9/093018.

[62] M. Metikoš-Huković and Z. Grubač, The growth kinetics of thin anodic wo3 films investigated by electrochemical impedance spectroscopy, Journal of Electroanalytical Chemistry 556, 167 (2003). 


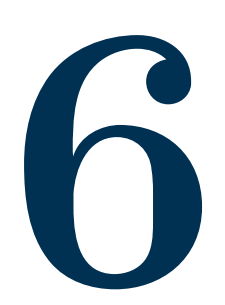

\section{YAMAMURA MODEL NEAR THE THRESHOLD: A Bayesian Analysis}

With four parameters I can fit an elephant, with five I can make him wiggle his trunk

John von Neumann

Semi-empirical formulae such as the Yamamura model provide a quick reference of sputter yields for applications of sputter depth profiling, low energy ion scattering, and secondary ion mass spectrometry applications. Scaling parameters used in such models are prone to errors which can propagate (linearly or non-linearly) into the prediction of sputter yields. For the Yamamura model, the deviation of predictions ( $\geq 20 \%$ ) was reduced (to $\sim 11 \%$ ) by corrections to the parameter $Q$ by Seah [Surface and Interface Analysis, 37, 5, 2005]. In this reference, $Q$ was found to scale with the density of the target material. We compare experimental sputter yields of transition metals with predictions of the Yamamura model using a Bayesian Markov Chain Monte Carlo (MCMC) algorithm. The fitting parameters $Q$ (defining linear scaling) and s (defining a power-law scaling) in the model for sputter yields are explored. The results from the MCMC for $Q$ are then compared with the propositions of Seah and extended to a collection of target materials by fitting simulations for argon and neon ion bombardment using the Monte Carlo code TRIDYN. Q values showed oscillatory behavior which is dependent on the energy transferred from the ion to the target atoms. 


\subsection{Introduction}

Ballistic processes involved in energetic ion - target surface interactions find applications in thin film physical-vapor deposition [1], space [2] and fusion [3] research. They are also widely exploited in metrology applications such as for sputter depth profiling [4], low-energy ion scattering [5, 6], and secondary ion mass spectroscopy. The interactions in each case lead to a removal of target material (sputtering). While this may be beneficial to some applications and detrimental to others, in either case, the quantification of sputtering efficiency is strongly desired. Errors in predictions could lead to a range of issues, starting with minor ones, such as deposition of thinner (or thicker) films which are relatively simple to correct. However, improper sputter efficiencies can also lead to major issues such as errors in quantification of material and sample depth in metrology applications, or to device failure during operation of materials exposed to plasmas [7].

The quantification of sputter yields has improved with growth of understanding of the nature of processes involved in ion-target interactions. Models accounting for energy losses from an ion into the metal via nuclear $[8,9]$ and electronic $[10,11]$ channels were developed along with inter-atomic potentials [9]. Sigmund first formulated a semi-empirical predictive equation for sputter yields [12]. Improvements to the model led to greater precision in predictability and extension of usage to low ion energies near the sputter threshold. Modern technology facilitated the use of computationally intensive methods like Monte Carlo codes [13, 14] which are capable of predicting not only sputter efficiencies, but also dynamic changes to the target composition.

Although complex computer aided predictions such as Monte Carlo codes have become available, semi-empirical formulae are still favored due to their simplicity. The accuracy of predictions for a given semi-empirical formula has also improved through an understanding of the various fitting parameters used. A significant step in improving this understanding was proposed by Seah $[15,16]$ for popular semiempirical models. Seah proposed the fitting parameter $Q$ - used in two popular predictive models, namely, the Yamamura [17] and Matsunami [18] models - to scale as a function of the inter-atomic spacing of the target (density). While this provided a physical basis to an otherwise fitting parameter, it was only applied to one ion species (argon) for the Yamamura model.

In this work, we shall look at the Yamamura model and its fitting parameters. We shall first study correlations between parameters by comparing the model to experimentally determined yields using a Bayesian parameter space searching algorithm. Subsequently, we shall compare the new fit parameter $Q$, with the improvements proposed in [15] in an effort to extend the application to more than one ion specie. Finally, we shall discuss a reformulation of the physical basis of $Q$ from a density term to an energy transfer term. 


\subsection{Semi-Empirical Formula}

\subsubsection{Yamamura Model}

Following the initial proposition by Sigmund [12] for predicting the sputter yield of a target under $>1 \mathrm{keV}$ ion bombardment, many developments to his propositions have evolved [17-20] for ion energies near the sputter threshold. One of the most extensively used semi-empirical models which we shall discuss in this chapter is the one developed by Yamamura [17]. It builds upon Sigmund's model and additionally accounts for electronic energy loss as well as a sputter threshold, and is defined as:

$$
Y(E)=0.042 \times \frac{Q\left(Z_{2}\right) \alpha^{*}\left(\frac{M_{1}}{M_{2}}\right)}{U_{S}} \frac{S_{n}(E)}{1+\Gamma k_{e} \epsilon^{0.3}}\left(1-\sqrt{\frac{E_{t h}}{E}}\right)^{s}
$$

where $Y$ is the sputter yield, which varies as a function of the incident ion energy, $E$ (in eV). $M_{1}$ and $M_{2}$ are the masses of the ion and target atoms (in amu), respectively. $\epsilon$ is the reduced energy. $\alpha^{*}$ is an energy independent function of the ratio of ion and target masses. $S_{n}$ is the nuclear stopping power of the target (in $\mathrm{eV} \AA^{2}$ atom $^{-1}$ ), $E_{t h}$ is the sputter threshold. $U_{S}$ is the surface binding energy, approximated by the sublimation energy of the target material. The factor 0.042 has units of $\AA^{-2}$. $\Gamma$ is a parameter that factors in the contribution of reflected ions to the recoil cascade and takes the form:

$$
\Gamma=\frac{W \epsilon^{-0.2}}{1+\left(M_{1} / 7\right)^{3}}
$$

where $W$ is taken from tabulated values (given for 32 elements) or is otherwise approximated as $W=0.35 \times U_{\mathrm{S}}$. $k_{e}$ is the correction factor for the electronic stopping power, set to 1 . A detailed treatise on the definitions and functional forms of parameters $\alpha^{*}, \mathrm{~S}_{n}(\mathrm{E})$ and $\epsilon$ can be found in [17]. $Q\left(Z_{2}\right), s$ and $E_{t h}$ are parameters that take values given by Yamamura in the form of lookup tables, with $E_{t h}$ being calculated as:

$$
E_{t h}= \begin{cases}\left(1+5.7 \frac{M_{1}}{M_{2}}\right) \frac{U_{S}}{\gamma}, & M_{1}<M_{2} \\ 6.7\left(\frac{U_{S}}{\gamma}\right), & M_{1}>M_{2}\end{cases}
$$

where $\gamma=4 M_{1} M_{2} /\left(M_{1}+M_{2}\right)^{2}$ is the energy transfer factor for elastic collisions. Unlike $E_{t h}$, the parameters $Q$ and $s$ do not have an empirical expression and their values need to be obtained for each target element. In most cases, $\mathrm{s}$ is fixed to a value of 2.5 , and occasionally changes to 2.8. $Q$, on the other hand, is considered to be dependent on the target only and is a function of its atomic number. However, no functional form was described in the work. Instead, $Q$ values were tabulated for 34 target materials by fitting experimental (and simulated) sputter yield data and using $Q$ as a fit parameter. Where data was unavailable (42 target elements), $Q$ was set to 1 .

It was shown by Seah [15], that this approximation of $Q$ leads to errors in prediction of sputter yields by at least $20 \%$ for targets where $Q$ was not tabulated 
(set to unity). These errors can be significant for applications such as sputter depth profiling, low energy ion scattering or secondary ion mass spectroscopy. In the following section, we shall look at the corrections proposed in [15] for the parameter $Q$ for argon ions.

\subsubsection{Corrections to Q: $\mathrm{Q}_{\mathrm{eff}}$}

In order to reduce the errors arising from unknown values of $Q$, the dependence of $Q$ on the target material was explored by Seah $[15,16]$. There, $Q$ was considered to have a dependence (and dimensions) of atomic density. To account for this dependence on the target density, an average inter-atomic spacing, r, was defined as:

$$
r^{3}=\frac{10^{24} M_{2}}{\rho N_{a v}} \quad\left(\mathrm{~nm}^{3}\right)
$$

where $N_{a v}$ is Avogadro's number and $\rho$ is the density of the target $\left(\mathrm{kg} / \mathrm{m}^{3}\right) . Q$, now termed $Q_{\text {eff }}$, is then allowed to scale with the mass and density of the target as:

$$
Q_{e f f}=r^{-3}\left[a\left(\exp \left(-\frac{\left(M_{2}-b\right)^{2}}{2 c^{2}}\right)\right)+d\left(1-\exp \left(-\frac{\left(M_{2}-e\right)^{2}}{2 f^{2}}\right)\right)\right]
$$

where $a, b, c, d, e$, and $f$ are fitting coefficients - with values: 0.0202, 19.0, $14.6,0.0166,5$, and 50, respectively for argon ion bombardment- that include any dependence of $Q$ on the ion specie. Albeit artificially (due to the coefficients), $Q_{\text {eff }}$ now scales only with the target parameters (mass and density). Equation 6.5 reduces the number of tabulated values from 77 in total (one for each target) to 6 per ion specie, while decreasing the relative error between predictions and experimental data to $9 \%$ on average. The Gaussian terms are used to describe the fluctuations in $Q$ as a function of target mass and provide a good approximation for elements with $M_{2} \leq 100$ for argon ion bombardment. In [15, 16], the coefficients in equation 6.5 were tabulated for argon for the Yamamura model. However, similar treatment was not performed for neon due to possible correlations of $Q_{e f f}$ with s and large $Q_{\text {eff }} r^{3}$ oscillations at low target masses.

We shall, in the following section, describe a probabilistic method for searching the parameter space while we simultaneously observe correlations between model parameters and quantify uncertainty in the parameters of the Yamamura model.

\subsection{Bayesian MCMC}

In order to assess the best-fit parameters and uncertainties therein caused by correlations, Bayesian Analysis is a powerful tool. Bayesian Analysis relies on probabilistic definitions based on Bayes' rule [21] which is given as:

$$
P(\Theta \mid D, M)=P(\Theta \mid M) \frac{P(D \mid \Theta, M)}{P(D \mid M)} \propto P(\Theta \mid M) P(D \mid \Theta, M)
$$


where $P(\Theta \mid D, M)$ is the posterior probability distribution function (PDF) which describes the probability of (the value of) a parameter set $\left(\Theta=\left\{\theta_{1}, \theta_{2}, \ldots, \theta_{k}\right\}\right)$ given a certain data set $\left(D=\left\{x_{1}, x_{2}, \ldots, x_{n}\right\}\right)$ for a model $(M)$. Here, $n$ are the number of data points and $k$ the number of model parameters. $P(\Theta \mid M)$ (read as $\Theta$ given $\mathrm{M}$ ) encodes the prior knowledge of the model parameters in predicting data, and is called the prior PDF. $P(D \mid \Theta, M)$ is the likelihood function $(\mathcal{L})$ which is defined later. The denominator, $P(D \mid M)$ is often termed as the Bayesian evidence, which refers to the validity of the model in predicting the data, and for practical purposes serves as a normalizing factor in equation 6.6.

Bayesian Markov Chain Monte Carlo (MCMC) allows for a means of estimating the posterior probability defined by Bayes' theorem by randomly sampling the PDFs of the likelihood, $\mathcal{L}$ and the prior. For an objective analysis, we assume no $a$ priori knowledge of the mean and distribution of the model parameters. This is mathematically described by the use of a uniform PDF as priors for each of the model parameters. When uniform priors are used, the posterior PDF is proportional to the likelihood function as:

$$
P(\Theta \mid D, M) \propto P(D \mid \Theta, M)=\mathcal{L}
$$

The random sampling is performed by using an affine invariant ensemble sampler, emcee [22]. The affine invariant sampler is advantageous over other common sampling methods $[23,24]$ as it is unperturbed by affine transformations (rotations, stretching) and multi-modality. This allows for sampling between parameters that vary over orders of magnitude. The sampling of the prior PDF occurs through assignment of a set (ensemble) of walkers. The walkers are k-dimensional vectors which randomly move across the parameter space. Their movement is governed by the likelihood function, $\mathcal{L}$, which for emcee is defined as a log-likelihood:

$$
\log (P(D \mid \Theta))=\log (\mathcal{L})=-\frac{1}{2} \sum_{i=1}^{n}\left[\left(2 \pi e_{i}\right)^{2}+\frac{\left(Y_{i}-Y_{\text {yama }}^{2}\right)}{e_{i}^{2}}\right]
$$

where $Y_{i}$ is the experimental yield with uncertainty $e_{i}$, and the subscript denotes the particular data count for $\mathrm{n}$ data points in a dataset D. $Y_{y a m a}$ is the yield predicted by the model (equation 6.1). The walkers move in a two-step process, first by selecting a point to jump to, and then deciding whether the jump occurs. The second step helps to map out the contours in the parameter space and the walkers cluster around locations of highest probability. The walker movement process is repeated thousands of times for each walker until the entire posterior PDF is mapped and the likelihood is maximized. As a result, the posterior PDF of each model parameter is populated by the walkers and correlations between parameters can be evaluated from the PDF maps. As a consequence of this analysis, the best-fit parameters and the confidence in their value can be readily obtained.

For purposes of our analysis, equation 6.1 was used as the model (M). The parameter space $\Theta$ was the set of free-parameters: $Q, s, E_{t h}$. All MCMC calculations were performed using an ensemble of 50 walkers for a step count of 25000 steps. 
The first 5000 steps were discarded (burn-in) to negate any bias introduced by the choice of starting positions of the ensemble. The priors were chosen to be uniformly distributed with the starting value equal to the tabulated values (for $Q$ and $s$ ) or as calculated using equation 6.3 .

\subsection{Experimental Method}

Sputter yields were obtained experimentally for transition metal films of molybdenum $(\mathrm{Mo})$, ruthenium $(\mathrm{Ru})$, palladium $(\mathrm{Pd})$ and tungsten $(\mathrm{W})$ by measuring mass loss from a quartz crystal micro-balance (QCM). Argon and neon ions were generated using a $15 \mathrm{~cm}$ DC Kaufman ion source (Veeco Instruments) and the ion flux was monitored using a Faraday cup, simultaneously along with the mass loss. The ion energies were pre-characterized by the Faraday cup which also served as a retarding field energy analyzer. Ion energies for obtaining sputter yield data were varied in random order between $50 \mathrm{eV}$ and $300 \mathrm{eV}$ to avoid systematic errors. For experimental details, we refer the reader to chapters 3 and 4 , and additionally to $[25]$.

\subsection{Results and Discussion}

\subsubsection{Experimental sputter yields and model parameters: $\mathrm{Ar}^{+}$}

Using $\mathrm{Ar}^{+}$as the bombarding ion specie, sputter yields were experimentally determined by measuring changes to the QCM frequency response. The results are shown in Figure 6.1. The data was compared to the predictions of the Yamamura model, replacing $Q$ with $Q_{\text {eff }}$ values from equations 6.4 and 6.5 using fit parameters $(a-f)$ from [15]. In addition, the experimental data was used to determine posterior probabilities for the parameters: $Q, s$ and $E_{t h}$ from the Yamamura model which then can be compared with values from equation 6.4 and 6.5. Availability and ion energy range of literature data on yields differ between elements $\mathrm{Ru}$, Mo, $\mathrm{Pd}$ and $\mathrm{W}$. In order to prevent varying credibility in the best-fit values from limited reference datasets, reference measurements were not used as inputs into the MCMC algorithm. We shall see in a later section (6.5.3), the comparison of the obtained posterior probabilities in comparison to literature datasets.

Experimentally, the sputter yields for molybdenum, ruthenium and tungsten are lower than reports from literature values obtained from experiments from bulk samples [26, 27] or e-beam deposited films [25]. This is possibly due to our sputter deposited films being denser (due to energetics of the deposition process) and more flat on a nanometer scale roughness, compared to materials previously studied in literature. However, this hypothesis is inconsistent as can be observed in the case of palladium films (Figure 6.1d) and as we shall see, in the case of $\mathrm{Ne}^{+}$ion bombardment. The dependence of sputter yields on structure, density and roughness and the interplay between them, therefore, appears to be a complex function of these parameters and requires dedicated experiments. Differences arising from these parameters would exist for every dataset (measured in this work and in literature) and precise understanding of this interplay is beyond the scope of this paper. We shall 

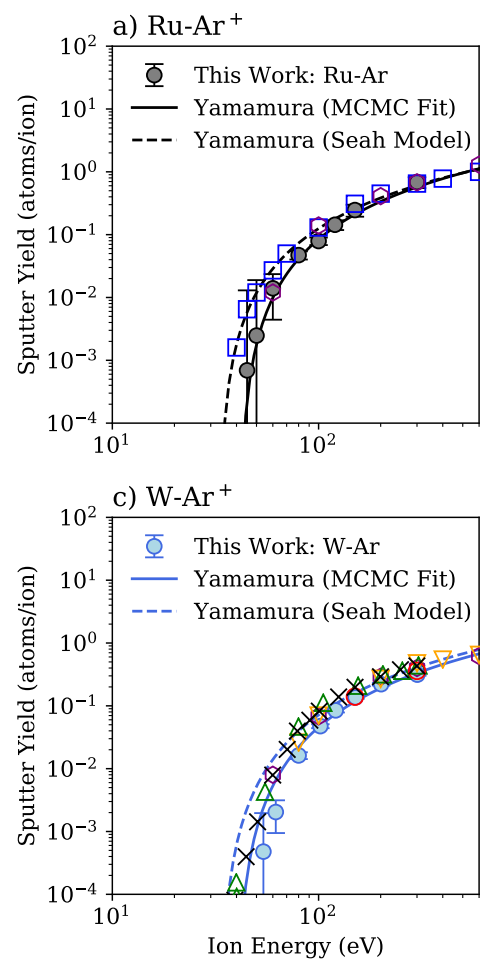

b) $\mathrm{Mo}^{-\mathrm{Ar}^{+}}$

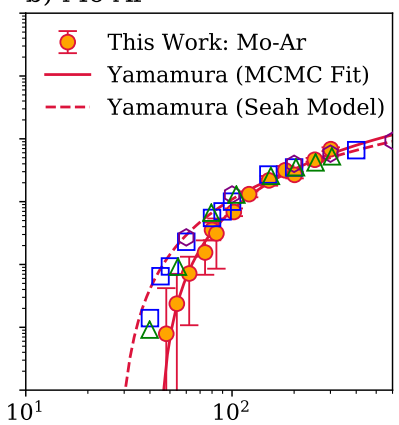

d) $\mathrm{Pd}-\mathrm{Ar}^{+}$

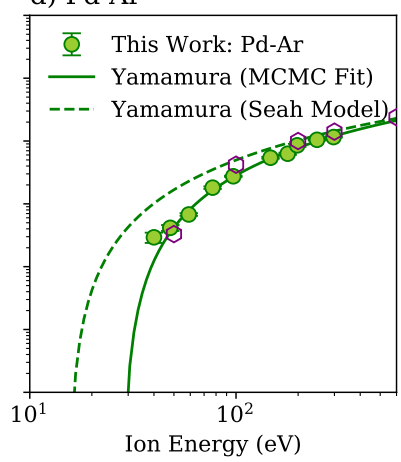

○ Laegreid, Wehner (1961)

$\square \quad$ Wu et. al (2009)

$\triangle$ Somogyvari, et al (2012)

$\nabla \quad$ Kolasinski et. al (2007)

Winters, Horne (1974)

$\times \quad$ Stuart, Wehner(1962)

Figure 6.1: Sputter Yields obtained under $\mathrm{Ar}^{+}$bombardment for a) ruthenium; b) molybdenum; c) tungsten; and d) palladium. Reference yields are also plotted (common legend out of plots). Fits to the Yamamura model using parameter values from the MCMC outputs (solid lines) are compared to Yamamura model with Seah's $Q_{\text {eff }}$ corrections (dashed lines).

instead focus on a global behavior of sputter yields. For this, we use the ensemble sampler described in section 6.3 for our dataset as well as a collection of available literature data (see Section 6.5.3).

An example of the posterior probabilities generated from the MCMC algorithm for palladium is shown in Figure 6.2. Similar plots which were generated for the other metals studied are shown in Appendix B. The MCMC maps depict a negative correlation for $E_{t h}$ with both $Q$ and $s$. However, $Q$ and $s$ are positively correlated with $Q$ straying to a $12 \%$ larger value from the $Q_{\text {eff }}$ calculated in [15], and $50 \%$ larger than the original tabulation of $Q$ in [17]. The threshold for palladium sputtering by argon is found to be $85 \%$ higher than that estimated by equation 6.3 . This relatively high value for $E_{t h}$ arises partly due to the lack of large sets of data below $\sim 50 \mathrm{eV}$. The power of the Bayesian method lies in the updating of posterior probabilities via better likelihood and prior estimates which are facilitated by availability of datasets. We shall compare the 'best fits' from our dataset with a compendium of literature values in Section 6.5.3.

Table 6.1 encapsulates the priors used and posteriors obtained in each of the datasets. It is evident that in each case, the threshold value depends on the avail- 


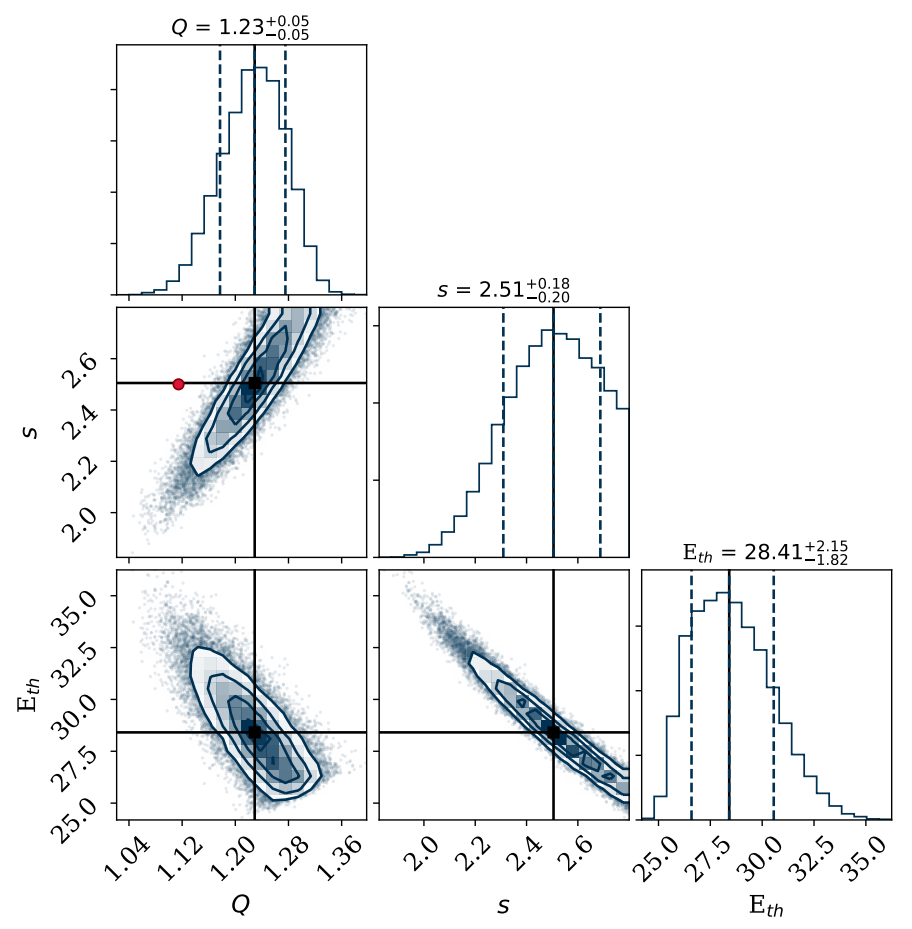

Figure 6.2: Posterior probability distributions for parameters from equation 6.1 for sputter yield data from palladium under $\mathrm{Ar}^{+}$bombardment. The red circle depicts $Q_{e f f}$ and $s$ values from [15]. The black cross-hairs show the 'best fit' locations on the contours and the slices are used to generate the probability distribution histograms on the diagonals. The contours depict $16^{\text {th }}, 50^{\text {th }}$, and $84^{\text {th }}$ quantiles.

ability of data near the true (yet unknown) threshold.

Table 6.1: Parameters used to search the posterior probabilities of the model parameters in equation 6.1. Sputter yields from $\mathrm{Ar}^{+}$bombardment used for likelihood estimates. The priors are described in the form: $\mathrm{U}$ (low, up), denoting uniform probabilities over a range with a lower (low) bound and an upper (up) bound. The maximum likelihood indicates the 'best fit' values obtained from the Bayesian sampling.

\begin{tabular}{cccccccccc}
\hline & \multicolumn{2}{c}{ Priors } & \multicolumn{3}{c}{ Maximum Likelihood } & \multicolumn{3}{c}{ Yamamura } \\
\hline \hline & $Q$ & $s$ & $E_{t h}$ & $Q$ & $s$ & $E_{t h}$ & $Q$ & $s$ & $E_{t h}$ \\
\hline $\mathrm{Mo}$ & $\mathrm{U}(0.17,4.25)$ & $\mathrm{U}(1.5,2.8)$ & $\mathrm{U}(20,45)$ & $1.58 \pm 0.11$ & $2.73 \pm 0.10$ & $43.9_{-1.36}^{+0.81}$ & 0.85 & 2.8 & 27.7 \\
$\mathrm{Ru}$ & $\mathrm{U}(0.26,6.55)$ & $\mathrm{U}(1.5,2.8)$ & $\mathrm{U}(10,40)$ & $1.35 \pm 0.33$ & $2.58_{-0.26}^{+0.16}$ & $41.4_{-3.54}^{+2.48}$ & 1.31 & 2.5 & 26.9 \\
$\mathrm{Pd}$ & $\mathrm{U}(0.17,4.25)$ & $\mathrm{U}(1.5,2.8)$ & $\mathrm{U}(5,45)$ & $1.23 \pm 0.05$ & $2.50 \pm 0.19$ & $28.4_{-1.83}^{+2.18}$ & 0.85 & 2.5 & 15.4 \\
$\mathrm{~W}$ & $\mathrm{U}(0.14,3.60)$ & $\mathrm{U}(1.5,2.8)$ & $\mathrm{U}(10,50)$ & $1.08 \pm 0.04$ & $2.78 \pm 0.03$ & $39.7_{-0.46}^{+0.23}$ & 0.72 & 2.8 & 33.9 \\
\hline
\end{tabular}

The $Q$ values obtained from the MCMC likelihood estimates, deviate from the $Q_{\text {eff }}$ proposed in [15] by at most $58 \%$ (molybdenum) and at least $3 \%$ (tungsten). 
Ensembles in some cases were observed to cluster around the upper bound due to instabilities possibly originating from the factor $\left(1-\sqrt{E_{t h} / E}\right)^{s}$ in equation 6.1 , which may become negative for certain s values, in case $E$ is below an assumed $E_{t h}$ in the algorithm.

\subsubsection{Experimental sputter yields and model parameters: $\mathrm{Ne}^{+}$}

Similar experiments using the QCM were carried out for the target elements under neon bombardment. The sputter yields obtained in this work along with references are plotted in Figure 6.3. Here, the structural dependence of the sputter yields is not apparent as the yields are consistent with reports in literature, taken from [26] and [27] for (bulk) samples immersed in a plasma.

In previously reported studies on the application of the Yamamura model for $\mathrm{Ne}^{+}$ion bombardment, an analysis of the dependence of $Q_{\text {eff }}$ on target mass was not performed [16]. The coefficients $(a-f$ in equation 6.5$)$ being unknown, we rely on the original $Q$ proposed by Yamamura for comparison. The Yamamura model in most cases provides suitable prediction for all elements down to $100 \mathrm{eV}$. Below $100 \mathrm{eV}$, the predictions begin to deviate from experiments.

Table 6.2: Parameters used to search the posterior probabilities of the model parameters in equation 6.1. Sputter yields from $\mathrm{Ne}^{+}$bombardment used for likelihood estimates. The priors are described in the form: U(low, up), denoting uniform probabilities over a range with a lower (low) bound and an upper (up) bound. The maximum likelihood indicates the 'best fit' values obtained from the Bayesian sampling.

\begin{tabular}{cccccccccc}
\hline & \multicolumn{3}{c}{ Priors } & \multicolumn{4}{c}{ Maximum Likelihood } & \multicolumn{3}{c}{ Yamamura } \\
\hline \hline & \multicolumn{1}{c}{$s$} & $E_{t h}$ & $Q$ & $s$ & $E_{t h}$ & $Q$ & $s$ & $E_{t h}$ \\
\hline $\mathrm{Mo}$ & $\mathrm{U}(0.17,4.25)$ & $\mathrm{U}(1.5,2.8)$ & $\mathrm{U}(5,40)$ & $0.85 \pm 0.04$ & $2.19_{-0.07}^{+0.15}$ & $35.01_{-2.06}^{+1.41}$ & 0.85 & 2.8 & 26.1 \\
$\mathrm{Ru}$ & $\mathrm{U}(0.26,6.55)$ & $\mathrm{U}(1.5,2.8)$ & $\mathrm{U}(5,40)$ & $1.35 \pm 0.01$ & $2.8 \pm 0.01$ & $30.46_{-0.04}^{+0.05}$ & 1.31 & 2.5 & 25.9 \\
$\mathrm{Pd}$ & $\mathrm{U}(0.17,4.25)$ & $\mathrm{U}(1.5,2.8)$ & $\mathrm{U}(5,40)$ & $1.26 \pm 0.05$ & $2.39_{-0.20}^{+0.26}$ & $25.0_{-2.99}^{+2.88}$ & 0.85 & 2.5 & 15.1 \\
$\mathrm{~W}$ & $\mathrm{U}(0.14,3.60)$ & $\mathrm{U}(1.5,2.8)$ & $\mathrm{U}(5,40)$ & $0.63 \pm 0.05$ & $2.34_{-0.17}^{+0.27}$ & $32.78_{-3.26}^{+2.91}$ & 0.72 & 2.8 & 40.6 \\
\hline
\end{tabular}

The MCMC sampling was performed much in the same way as for argon sputter yields. A uniform probability distribution between certain bounds was chosen for the parameters as priors. The results of the ensemble sampling for palladium sputtered by $\mathrm{Ne}^{+}$are shown in figure 6.4. The positive correlation of $s$ with both $Q$ and $E_{t h}$ is significantly reduced for this dataset. $E_{t h}$ and $s$, which were previously negatively correlated (for $\mathrm{Ar}^{+}$) still behave similarly, but the correlation is weaker. The histogram of $s$ shows a near uniform probability distribution for $s$ in the region chosen, which is slightly skewed due to its dependence on $Q$ and $E_{t h}$.

The initial inputs and the maximum likelihood obtained are summarized in table 6.2 along with propositions by Yamamura. We notice that the sputter threshold from the maximum likelihood estimate is - for molybdenum, ruthenium and palladium - lower than as predicted by equation 6.3. Tungsten, on the other hand, showed a lower experimental threshold which is accounted for by the MCMC parameter search. We also find that the energy scaling given by the exponent $s$, varies 

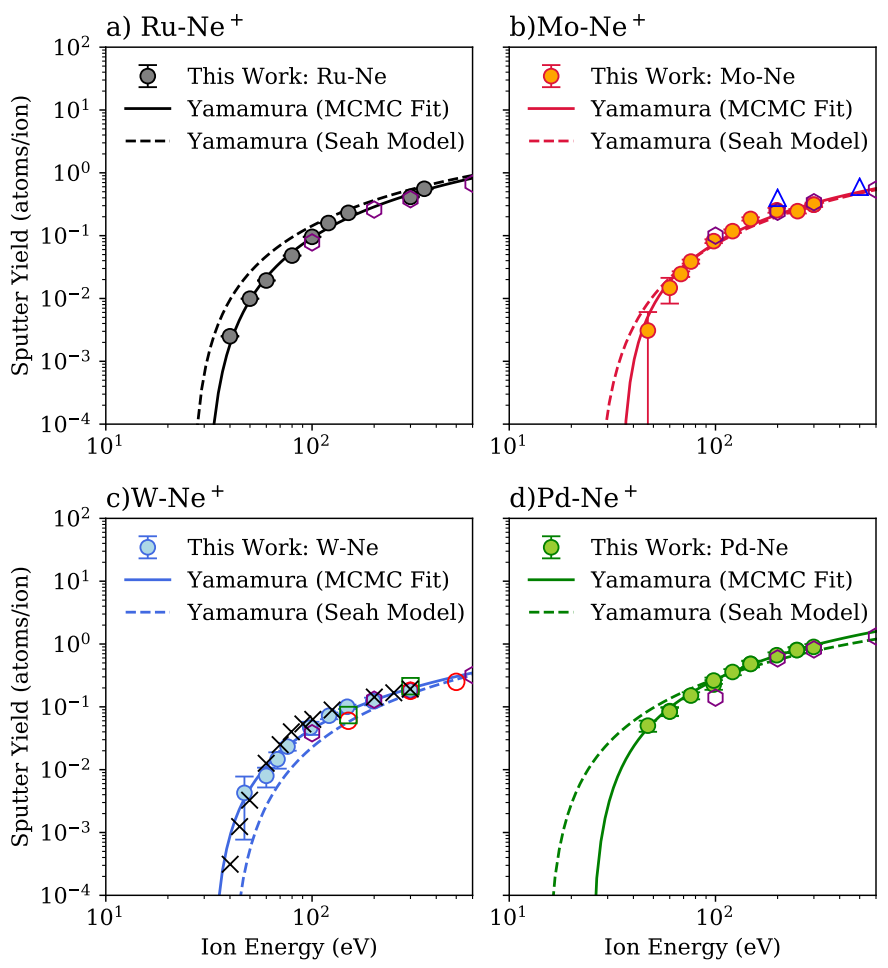

- Laegreid, Wehner (1961)

$\times \quad$ Stuart, Wehner (1962)

$\square$ Hechtl, Bohdansky (1981)

○ Winters, Horne (1974)

$\triangle$ Zalm (1983)

Figure 6.3: Sputter Yields obtained under $\mathrm{Ne}^{+}$bombardment for a) ruthenium; b) molybdenum; c) tungsten; and d) palladium. Reference yields are also plotted (common legend out of plots). Fits to the Yamamura model using parameter values from the MCMC outputs (solid lines) are compared to Yamamura model.

between elements and is correlated to $Q$ (positively) and $E_{t h}$ (negatively). This change in $s$, is restricted mostly to either the initial tabulation by Yamamura, or varies in the vicinity of one of the tabulated values (2.5 or 2.8). However, in cases as molybdenum sputtering by neon, s moves to a lower value and the scaling of the data moving from near-threshold to higher energies is represented well. In order to determine the nature of $s$ and its scaling as a function of energy, experimental data for each element needs to be considered.

We find that the maximum likelihood estimates for $Q$ represent the experimental data well but in some cases deviate from the predictions of $Q_{\text {eff }}$ in equation 6.5 and Yamamura's tabulations as is seen in figures 6.2 and 6.4, respectively. The elements studied here are heavy $\left(M_{2} \sim 100\right.$ or greater). Equation 6.5 predicts for heavy elements, no change in $Q_{\text {eff }}$ other than that induced by the inter-atomic spacing. In the following section we shall compare $Q$ obtained from MCMC ensemble sampling to the predictions of equation 6.5 using reference data for a multitude of elements where experimental data is available. 


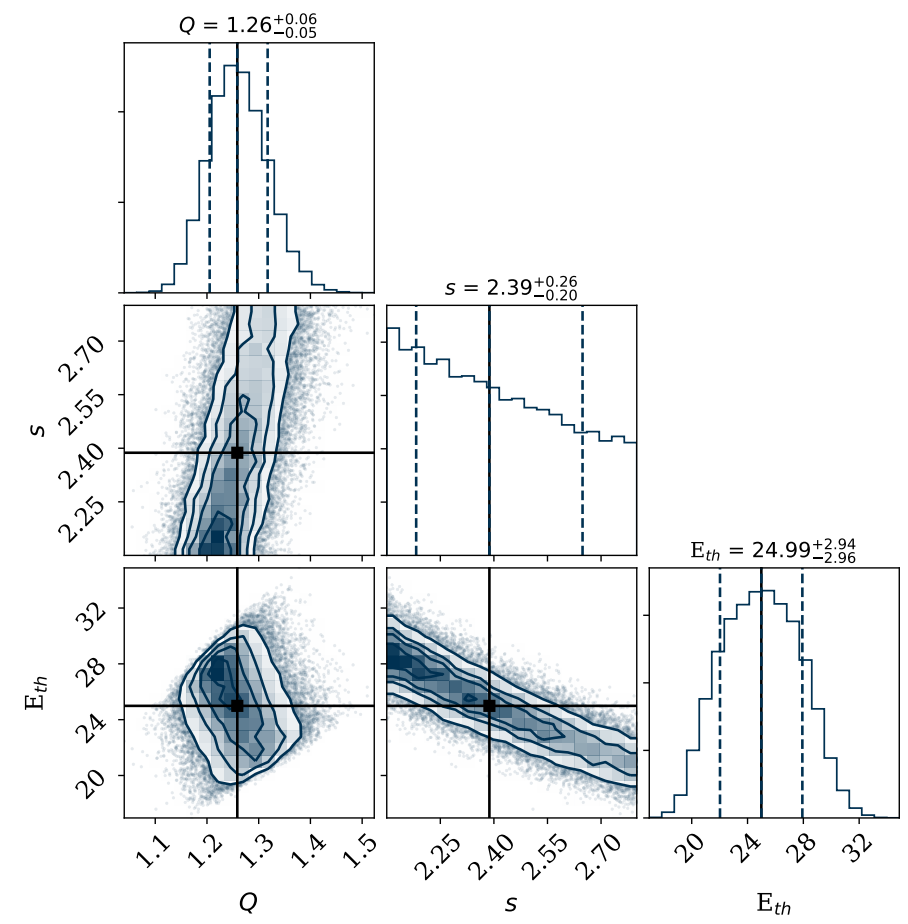

Figure 6.4: Posterior probability distributions for parameters from equation 6.1 for sputter yield data from palladium under $\mathrm{Ne}^{+}$bombardment. The black cross-hairs show the 'best fit' locations on the contours and the slices are used to generate the probability distribution histograms on the diagonals. The contours depict $16^{\text {th }}, 50^{\text {th }}$, and $84^{\text {th }}$ quantiles.

\subsection{3 $\mathrm{Q}_{\mathrm{MCMC}}$ and $\mathrm{Q}_{\mathrm{eff}}$}

In section 6.5.1 and 6.5.2, we found that the values of $Q$ and $s$ are correlated using the MCMC ensemble sampling method. While the values of $\mathrm{s}$ were found to vary from one element to the other, and sometimes inverted from the initial proposition, the initial tabulations of 2.5 or 2.8 in the Yamamura model were found to be reasonable in most cases. The linear scaling factor $Q$ ( $Q_{M C M C}$ in our study), requires some scrutiny as its effect on the sputter yield is relatively clear. Here, we begin by comparing the obtained $Q_{M C M C}$ with $Q_{\text {eff }}$ from $[15,16]$. Equation 6.5 predicts that the term $Q_{e f f} r^{3}$ for argon bombardment saturates to a constant value for target masses $>100 \mathrm{amu}$. This plateau, while apparent in the evaluation in $[15,16]$, would be unlikely as elemental properties are periodic and any initial fluctuation in $Q_{e f f} r^{3}$ at low target masses would carry forward to high masses as well. In the evaluation, experimental sputter yield datasets for a variety of target materials were studied, however, there was a lack of populated data for the region between $110 \mathrm{amu}$ and $180 \mathrm{amu}$. Any fluctuations, if present in this region, would flatten out for the higher target masses.

Here we use experimental data available for a variety of elements obtained from 
the literature [25, 26, 28-33]. To supplement the lack of sputter yield data for target masses between 110 - $180 \mathrm{amu}$ (d and f block elements), we chose to simulate the yields for a selection of lanthanides using TRIDYN [13]. Further, to understand the limitation/validity of this simulation based approach, we randomly selected elements for which literature data was available and used them as inputs for the MCMC ensemble sampler. We performed this analysis for sputter yield data from argon and neon ion bombardment.
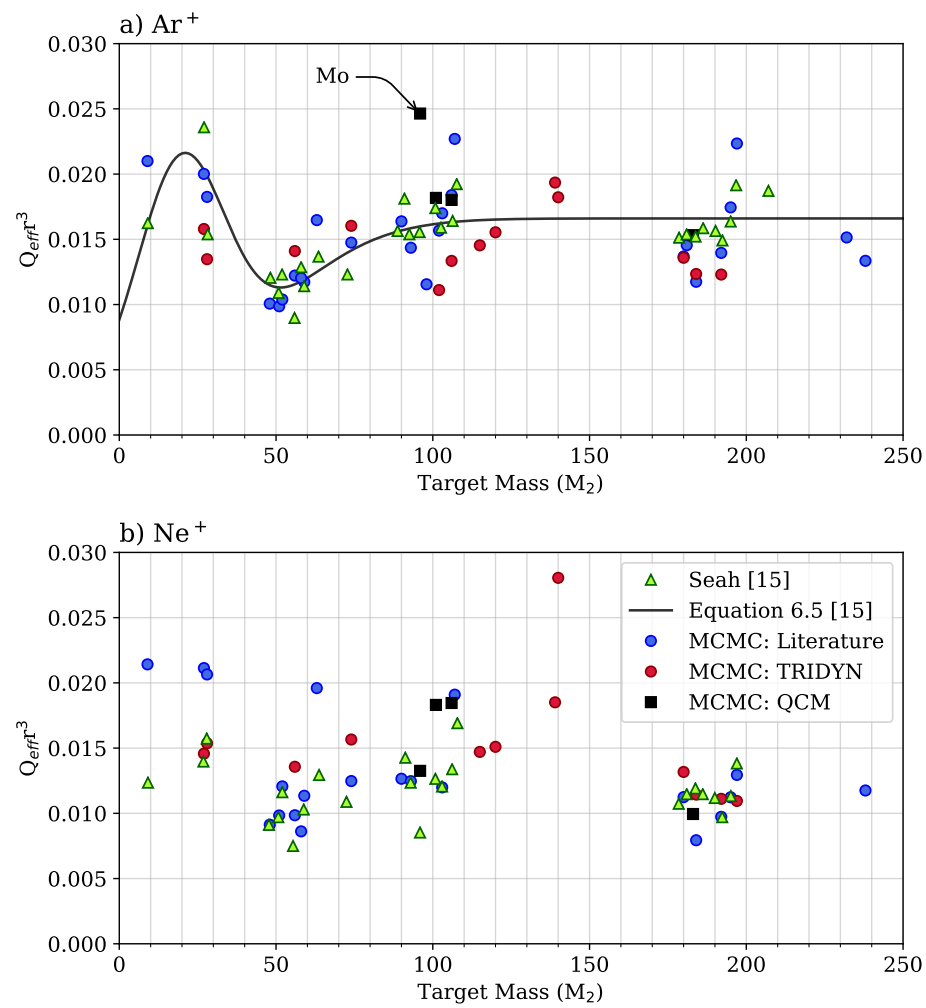

Figure 6.5: $Q_{\text {eff }}$ from [16] (green triangles) along with corresponding $Q$ values obtained using the MCMC algorithm for: literature (blue), QCM experiments from this work (black squares) and TRIDYN simulations in this work (red) for a) $\mathrm{Ar}^{+}$and b) $\mathrm{Ne}^{+} . Q_{e f f}$ values from equation 6.5 with coefficients $(a, b, c, d, e, f)=(0.0202,19,14.6,0.0166,5,50)$ for $\mathrm{Ar}^{+}$also plotted (black line). Similar values of parameters for Ne not available.

Figure 6.5a depicts the maximum likelihoods of $Q_{M C M C}$ for experimental sputter yields (labeled literature), and for simulated yields for selected targets under $\mathrm{Ar}^{+}$ion bombardment. For comparison, $Q_{\text {eff }}$ from [15] are also plotted along with the predictive function in equation 6.5. While the scatter in the datasets from MCMC ensemble sampling is quite large, the behavior of $Q_{M C M C}$ is generally consistent with equation 6.5 for target masses $<100 \mathrm{amu}$. For $M_{2}>100 \mathrm{amu}$, the initial oscillation (in the region of $20 \leq M_{2} \leq 70 \mathrm{amu}$ ), repeats and continues on upto 250 amu. The periodicity is non-uniform and is not captured by equation 6.5, which 
in this range, approximates the variation to a constant. $Q_{M C M C}$ from $\mathrm{Mo}-\mathrm{Ar}^{+}$ results from QCM measurements, marked in Figure 6.5a, is an outlier. A significant scatter is seen for data-points between $100 \mathrm{amu}$ and $110 \mathrm{amu}$, and the cause is yet unknown, however, an overall oscillatory behavior is observed over the range of target masses.

Data for neon bombardment is more scattered as seen in Figure 6.5b. Results not only from our MCMC analysis, but also reports from [16] show elevated scatter in comparison to the argon data. Although a structure for neon datasets similar to argon can be argued, the scatter in the data prevents such a conclusive remark.

In general, considering the oscillatory behavior of data, the two Gaussian terms in equation 6.5 do not fully encapsulate $Q_{M C M C}$ or $Q_{\text {eff }}$ for $\mathrm{M}_{2}>100 \mathrm{amu}$. A multitude of Gaussians would be needed under the approach of equation 6.5 which becomes cumbersome with the addition of more terms. We shall discuss in the following section an alternative perspective to the evaluation of the datasets in an effort to encapsulate the behavior of both ion species.

\section{Dependence on energy transfer}

The proposition of equation 6.5 was a significant step forward in determining an empirical relation to the scaling factor, $Q$, used in a multitude of predictive models of sputter yields. Although it was shown [15, 16] to be valid for models such as the Matsunami formula [18] (not discussed here), the approach was found to be insufficient for the Yamamura model due to the scatter in the data other than for argon (in addition to correlations between fit parameters). Further, the formulation of equation 6.5 is empirical and contrasts with the original formulation by Sigmund [12], which was also pointed out in [15].

We begin by rescaling the data on the abscissa. $U_{S}$ was considered as a choice of independent variable as proposed in [34], but no clear dependence of $Q$ on $U_{S}$ was observed in our datasets. Instead, empirical evidence suggests the value of $Q$ to depend on the choice of ion-target combinations. Sputtering is a process governed by energy transfer from an ion to a target atom. For comparing results from a heavy ion $\left(\mathrm{Ar}^{+}\right)$to those of a light ion $\left(\mathrm{Ne}^{+}\right)$, where energy is transferred with varying efficiency, a physical property from either the ion or target is insufficient. In the spirit of [20], $Q$ was chosen to depend on both the ion and target masses through the energy transfer parameter, $\gamma$, where $\gamma=4 M_{1} M_{2} /\left(M_{1}+M_{2}\right)^{2}$. Plotting $\log \left(Q^{\prime}\right)$, where $Q^{\prime}=\left\{Q_{M C M C}, Q_{e f f}\right\}$, as a function of $\gamma$ gives rise to irregularly spaced bands as shown in Figure 6.6. $Q_{\text {eff }}$ here is taken from [15] as shown in Figure 6.5 after dividing by $r^{3}$ from equation 6.4, thus allowing for direct comparison of $Q$ values from different sources. The bands show a periodicity similar to the irregular oscillations observed in the properties of elements (density, ionization energy, covalent radius). Argon data shows clear peaks and valleys, with peaks marked by elements with completely filled d-orbitals. As the d-orbital filling is complete, and a $\mathrm{p}$ or $\mathrm{f}$ orbital is filled, $\log \left(Q^{\prime}\right)$ drops into a valley and proceeds to increase with increasing electron filling for the orbital. The oscillations of $Q^{\prime}$ thus, seem dependent not directly on the density of the target element, but on the efficiency of energy transfer with patterns dictated by the filling of atomic orbitals. $Q_{M C M C}$ from $\mathrm{Mo}-\mathrm{Ar}^{+}$results from QCM measurements in this work is an outlier in both 
Table 6.3: Values of parameters used to describe $Q$ data in equation 6.9 for argon and neon ion bombardment.

\begin{tabular}{l|cccccc}
\hline & \multicolumn{6}{|c}{ Parameters } \\
\hline \hline & $a$ & $b$ & $c$ & $d$ & $e$ & $f$ \\
\hline Equation 6.9: $\mathrm{Ar}^{+}$ & 2.05 & 4 & 3.24 & 0.03 & -0.16 & - \\
Equation 6.9: $\mathrm{Ne}^{+}$ & 1.55 & 4 & 3.63 & -2.3 & -0.15 & - \\
\hline
\end{tabular}

descriptions of $Q\left(Q_{e f f}\right.$ and $\left.Q_{M C M C}\right)$ as seen in Figure 6.5 and Figure 6.6 justifying our approach to fit literature data and QCM results presented here as separate datasets. Again here, the neon data appears more noisy and scattered, however, the oscillations are evident (albeit with greater outliers) and similar arrangement of elements is observed. In order to describe the periodic bands of $Q^{\prime}$, a functional form of the periodicity of elements is required.

As a quick aside, the properties of elements in the periodic table have been studied to a great extent, but scarce (and complex) mathematical formulations exist $[35,36]$. Of the few mathematical formulations proposed, fewer are general purpose and simple to apply. We borrow a functional form from the proposition given in [37] and reduce it to:

$$
\log \left(Q^{\prime}\right)=b\left[\left\{c \gamma^{a}+d\right\}-0.5\right]^{2}+e
$$

through which $Q^{\prime}$ is expressed as a functional form of gamma leading to a universal expression that can be applied to multiple ion species, and doing so in a more physical manner (retaining periodicity).The parameters $a-e$ are used for fitting the $Q$ data from experiments, literature and Monte Carlo simulations to equation 6.9. It should be noted here, the use of the parenthesis $\{g\}$ acts towards rejecting the integer value produced by any function $g$, within it. For example, if $g$ produces values such as $1.5,1.8$, and 2.1 , then $\{g\}$ reduces them to $0.5,0.8$, and 0.1 , respectively. Equation 6.9 is in general, a functional description for the periodicity of properties of elements in the periodic table. The parameters take various values depending on the property being studied.

Using a non-linear least square fitting, a reasonable agreement can be obtained for both the argon and neon datasets. A summary of the parameters used to describe the data is provided in table 6.3. The number of parameters have effectively been reduced from 6 in equation 6.5 to 5 while describing a majority of the datasets. While the quality of the fit can still be improved through addition of another parameter for the exponent (here fixed to 2), in equation 6.9, the current results are reasonable. Before this perspective can be useful in predictability and application, some limitations need to be addressed.

\section{Limitations}

The current approach of describing the parameter $Q$ is limited by the resolution and quality of sputter yield data available. Literature data on sputter yields is scarce for energies below $200 \mathrm{eV}$. This causes large uncertainties between the $Q$ ( $s$ and $E_{t h}$ ) obtained from fitting reference datasets and simulated data. In our 

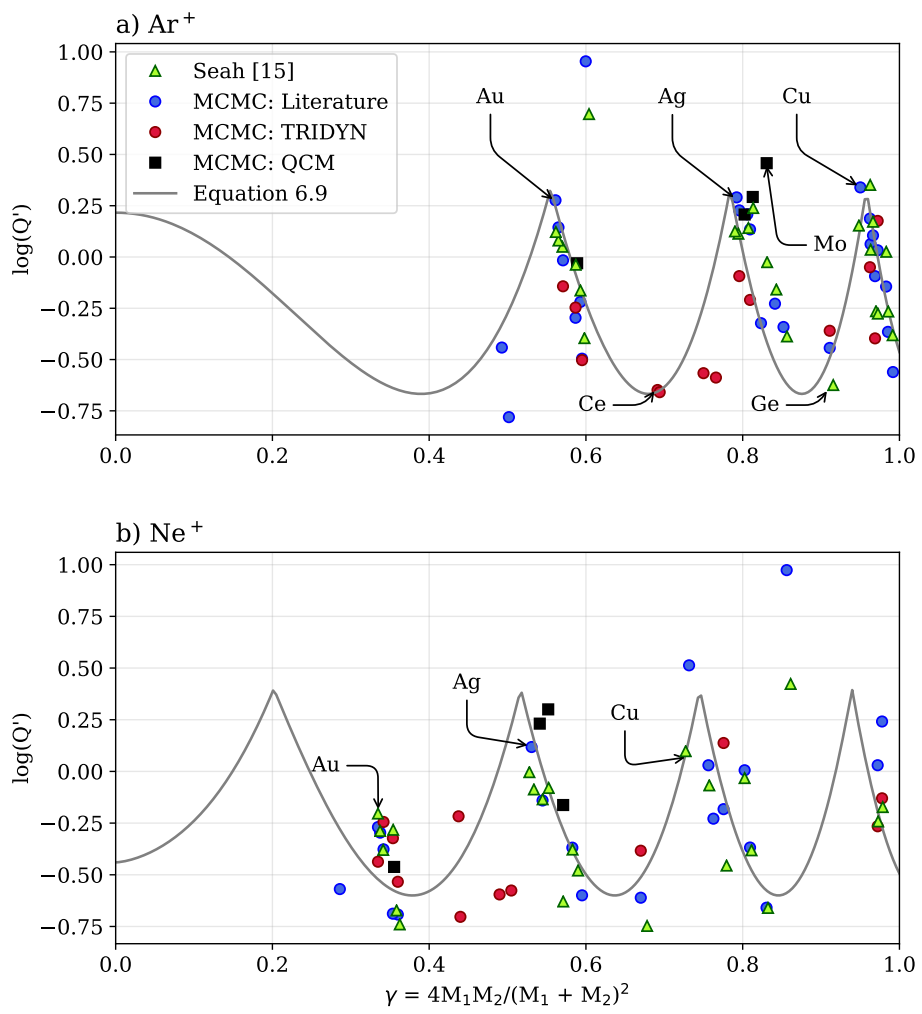

Figure 6.6: Values of $\mathrm{Q}$ from all datasets $Q^{\prime}=\left\{Q_{\mathrm{MCMC}}, Q_{\mathrm{eff}}\right\}$, plotted against the energy transfer parameter in elastic collisions for a) $\mathrm{Ar}^{+}$datasets and b) $\mathrm{Ne}^{+}$datasets. The bands clearly seen in a) show peaks for elements with a nearly fully filled d-orbital. Legends are the same as in Figure 6.5.

approach, some (rational) values of $s$, led to spurious and unpredictable behavior of equation 6.1 and thus, the MCMC algorithm. A more constrained, or reformulated implementation of the Yamamura model for the purpose of an MCMC analysis can be a potential solution. The non-linear least squares of equation 6.9 is possibly at a local minimum due to the periodic nature of the function, and more optimal parameters can be obtained through more conservative constraints. An additional parameter can be introduced to allow better flexibility of equation 6.9 to describe the data through the added degree-of-freedom. Finally, the periodicity of properties of elements can possibly be described in a functional form other than equation 6.9 which potentially has multiple (non-unique) solutions.

\subsection{Conclusions}

Experimental sputter yields near-sputter thresholds, cannot be quantitatively estimated a-priori using predictive models due to errors in scaling of the yields. We have investigated the parameters within the Yamamura sputter yield model using 
Bayesian Markov Chain Monte Carlo ensemble sampling. Following an experimental determination of sputter yields for heavy $\left(M_{2}>100 \mathrm{amu}\right)$ transition metals, namely molybdenum, ruthenium, palladium and tungsten, we apply the MCMC sampling to observe correlations between the fitting parameters. We further explore the scaling parameter $Q$ in the Yamamura model for a wide array of target materials by relying on available experimental data and simulations using TRIDYN. Upon comparing $Q_{M C M C}$ with previously reported $Q_{e f f}$ values, we find oscillations in $Q_{M C M C}$ as a function of target mass. These oscillations, similar to those of $Q_{e f f}$ at low $M_{2}$ values also progress for higher target masses. The values of $Q_{M C M C}$ or $Q_{M C M C}$ for both argon and neon ion bombardment, when plotted against the energy transfer parameter $\gamma$ showed clear peaks and valleys with fully filled d-orbital elements comprising the peaks and p-block and f-block elements comprising the valleys. An analytical expression for the oscillations of $Q$ is suggested allowing for estimation of $Q$ values based on energy transfer parameters applicable for both argon and neon. This potentially can be used to predict sputter yields of low energy ion bombardment with improved scaling for materials where data is still unavailable. 


\section{References}

[1] B. Krause, D. S. Kuznetsov, A. E. Yakshin, S. Ibrahimkutty, T. Baumbach, and F. Bijkerk, In situ and real-time monitoring of structure formation during non-reactive sputter deposition of lanthanum and reactive sputter deposition of lanthanum nitride, Journal of Applied Crystallography 51, 1013 (2018).

[2] M. K. Pospieszalska and R. E. Johnson, Magnetospheric ion bombardment profiles of satellites: Europa and Dione, Icarus 78, 1 (1989).

[3] K. Dobes, V. Smejkal, T. Schäfer, and F. Aumayr, Interaction between seeding gas ions and nitrogen saturated tungsten surfaces, International Journal of Mass Spectrometry 365-366, 64 (2014).

[4] K. Wittmaack, Beam-induced broadening effects in sputter depth profiling, Vacuum 34, 119 (1984), vacuum Special Issue: Proceedings of the 3rd International Conference on Low Energy Ion Beams Loughborough University of Technology, UK.

[5] H. Brongersma, M. Draxler, M. de Ridder, and P. Bauer, Surface composition analysis by low-energy ion scattering, Surface Science Reports 62, 63 (2007).

[6] C. R. Stilhano Vilas Boas, J. M. Sturm, and F. Bijkerk, Oxidation of metal thin films by atomic oxygen: A low energy ion scattering study, Journal of Applied Physics 126, 155301 (2019).

[7] J. Whitley, K. Wilson, and D. Buchenauer, Metallic materials as plasma facing components - a review, Journal of Nuclear Materials 155-157, 82 (1988).

[8] J. F. Ziegler and J. P. Biersack, The stopping and range of ions in matter, in Treatise on Heavy-Ion Science: Volume 6: Astrophysics, Chemistry, and Condensed Matter, edited by D. A. Bromley (Springer US, Boston, MA, 1985) pp. 93-129.

[9] W. D. Wilson, L. G. Haggmark, and J. P. Biersack, Calculations of nuclear stopping, ranges, and straggling in the low-energy region, Physical Review B 15, 2458 (1977).

[10] J. Lindhard and M. Scharff, Energy dissipation by ions in the kev region, Phys. Rev. 124, 128 (1961).

[11] O. S. Oen and M. T. Robinson, Computer studies of the reflection of light ions from solids, Nuclear Instruments and Methods 132, 647 (1976).

[12] P. Sigmund, Theory of sputtering. i. sputtering yield of amorphous and polycrystalline targets, Phys. Rev. 184, 383 (1969).

[13] W. Möller, W. Eckstein, and J. P. Biersack, Tridyn-binary collision simulation of atomic collisions and dynamic composition changes in solids, Computer Physics Communications 51, 355 (1988). 
[14] W. Eckstein, R. Dohmen, A. Mutzke, and R. Schneider, SDTrimSP version 5.00, IPP Report 12/08, Max- Planck-Institut für Plasmaphysik (Hrsg.) 12 (2007), http://edoc.mpg.de/display.epl? $\operatorname{mode}=$ doc\&id $=552734$.

[15] M. P. Seah, C. A. Clifford, F. M. Green, and I. S. Gilmore, An accurate semi-empirical equation for sputtering yields $i$ : for argon ions, Surface and Interface Analysis 37, 444 (2005), https://onlinelibrary.wiley.com/doi/pdf/10.1002/sia.2032 .

[16] M. Seah, An accurate semi-empirical equation for sputtering yields, ii: for neon, argon and xenon ions, Nuclear Instruments and Methods in Physics Research Section B: Beam Interactions with Materials and Atoms 229, 348 (2005).

[17] Y. Yamamura and H. Tawara, Energy dependence of ion-induced sputtering yields from monatomic solids at normal incidence, Atomic Data and Nuclear Data Tables 62, 149 (1996).

[18] N. Matsunami, Y. Yamamura, Y. Itikawa, N. Itoh, Y. Kazumata, S. Miyagawa, K. Morita, and R. Shimizu, A semiempirical formula for the energy dependence of the sputtering yield, Radiation Effects 57, 15 (1981).

[19] W. Eckstein, Sputtering yields, Vacuum 82, 930 (2008).

[20] J. Bohdansky, J. Roth, and H. L. Bay, An analytical formula and important parameters for low energy ion sputtering, Journal of Applied Physics 51, 2861 (1980).

[21] T. Bayes, An Essay Towards Solving a Problem In The Doctrine Of Chances, Biometrika 45, 296 (1958).

[22] D. Foreman-Mackey, D. W. Hogg, D. Lang, and J. Goodman, emcee: The mcmc hammer, Publications of the Astronomical Society of the Pacific 125, 306-312 (2013).

[23] N. Metropolis, A. W. Rosenbluth, M. N. Rosenbluth, A. H. Teller, and E. Teller, Equation of state calculations by fast computing machines, The Journal of Chemical Physics 21, 1087 (1953).

[24] J. Skilling, Nested sampling, AIP Conference Proceedings 735, 395 (2004).

[25] S. M. Wu, R. W. E. van de Kruijs, E. Zoethout, and F. Bijkerk, Sputtering yields of Ru, Mo, and Si under low energy Ar+ bombardment, Journal of Applied Physics 106, 054902 (2009).

[26] N. Laegreid and G. K. Wehner, Sputtering yields of metals for ar + and ne+ ions with energies from 50 to 600 ev, Journal of Applied Physics 32, 365 (1961).

[27] H. F. Winters and D. Horne, Energy transfer to a tungsten lattice by ion bombardment, Phys. Rev. B 10, 55 (1974). 
[28] P. C. Zalm, Energy dependence of the sputtering yield of silicon bombarded with neon, argon, krypton, and xenon ions, Journal of Applied Physics 54, 2660 (1983).

[29] Z. Somogyvári, G. A. Langer, G. Erdélyi, and L. Balázs, Sputtering yields for low-energy Ar + and $\mathrm{Ne}+$ ion bombardment, Vacuum 86, 1979 (2012).

[30] R. V. Stuart and G. K. Wehner, Sputtering yields at very low bombarding ion energies, Journal of Applied Physics 33, 2345 (1962).

[31] R. D. Kolasinski, J. E. Polk, D. Goebel, and L. K. Johnson, Sputtering yield measurements at glancing incidence using a quartz crystal microbalance, Journal of Vacuum Science \& Technology A 25, 236 (2007).

[32] R. Ranjan, J. P. Allain, M. R. Hendricks, and D. N. Ruzic, Absolute sputtering yield of ti/ tin by ar $+/ n+$ at 400-700 ev, Journal of Vacuum Science \& Technology A 19, 1004 (2001).

[33] K. Ikuse, S. Yoshimura, K. Hine, M. Kiuchi, and S. Hamaguchi, Sputtering yields of au by low-energy noble gas ion bombardment, Journal of Physics D: Applied Physics 42, 135203 (2009).

[34] G. Chambers and J. Fine, Practical surface analysis vol 2: Ion and neutral spectroscopy ed d briggs and $m p$ seah, (1992).

[35] R. Hakala, The periodic law in mathematical form. The Journal of Physical Chemistry 56, 178 (1952).

[36] G. Restrepo and L. Pachón, Mathematical aspects of the periodic law, Foundations of Chemistry 9, 189 (2007).

[37] N. S. Imyanitov, Modification of various functions for description of periodic dependences, Russian Journal of Coordination Chemistry 29, 46 (2003). 



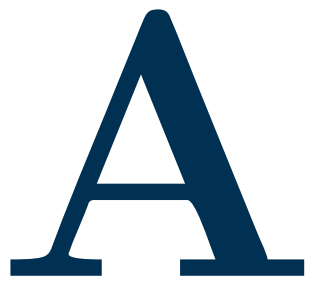

\section{ION BEAM NitRIDATION AND OXIDATION EFFECTS}

Here we include results of surface morphology induced by treatment of thin films studied in chapters 4 and 5. 


\section{A.1 Nitrogen ion beam effects}
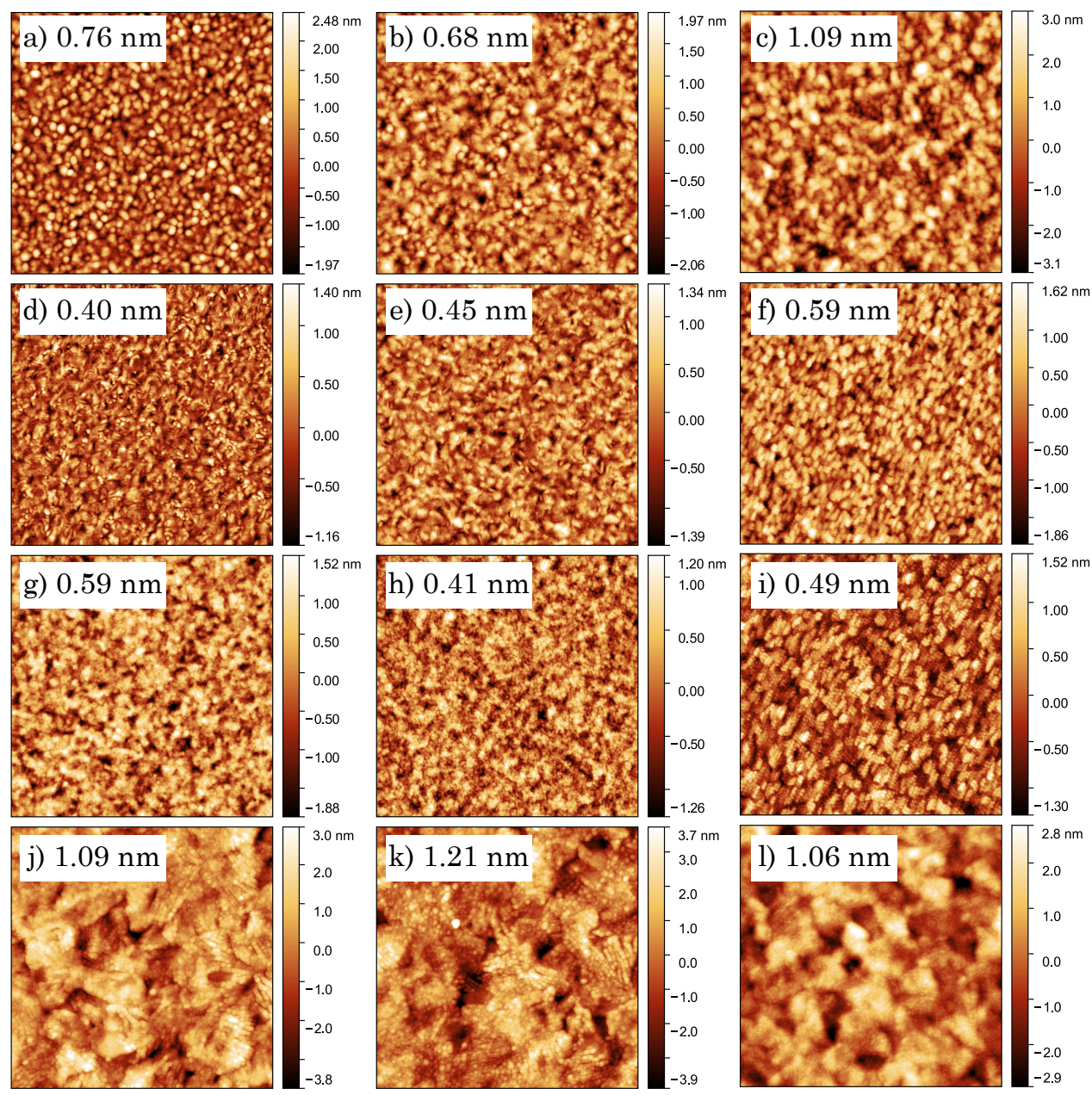

\section{$400 \mathrm{~nm}$}

Figure A.1: $1 \mu m \times 1 \mu m$ atomic force micrographs of (a-c) palladium; (d-f) ruthenium; (g-i) molybdenum; and $(\mathrm{j}-\mathrm{l})$ tungsten. The micrographs show, in increasing order: reference; $60 \mathrm{eV}$ $\mathrm{N}_{2}^{+} / \mathrm{N}^{+}$ion exposed and; $480 \mathrm{eV}\left(280 \mathrm{eV}\right.$ for palladium) $\mathrm{N}_{2}^{+} / \mathrm{N}^{+}$ion exposed surfaces. 


\section{A.2 Oxygen Ion Beam Effects}
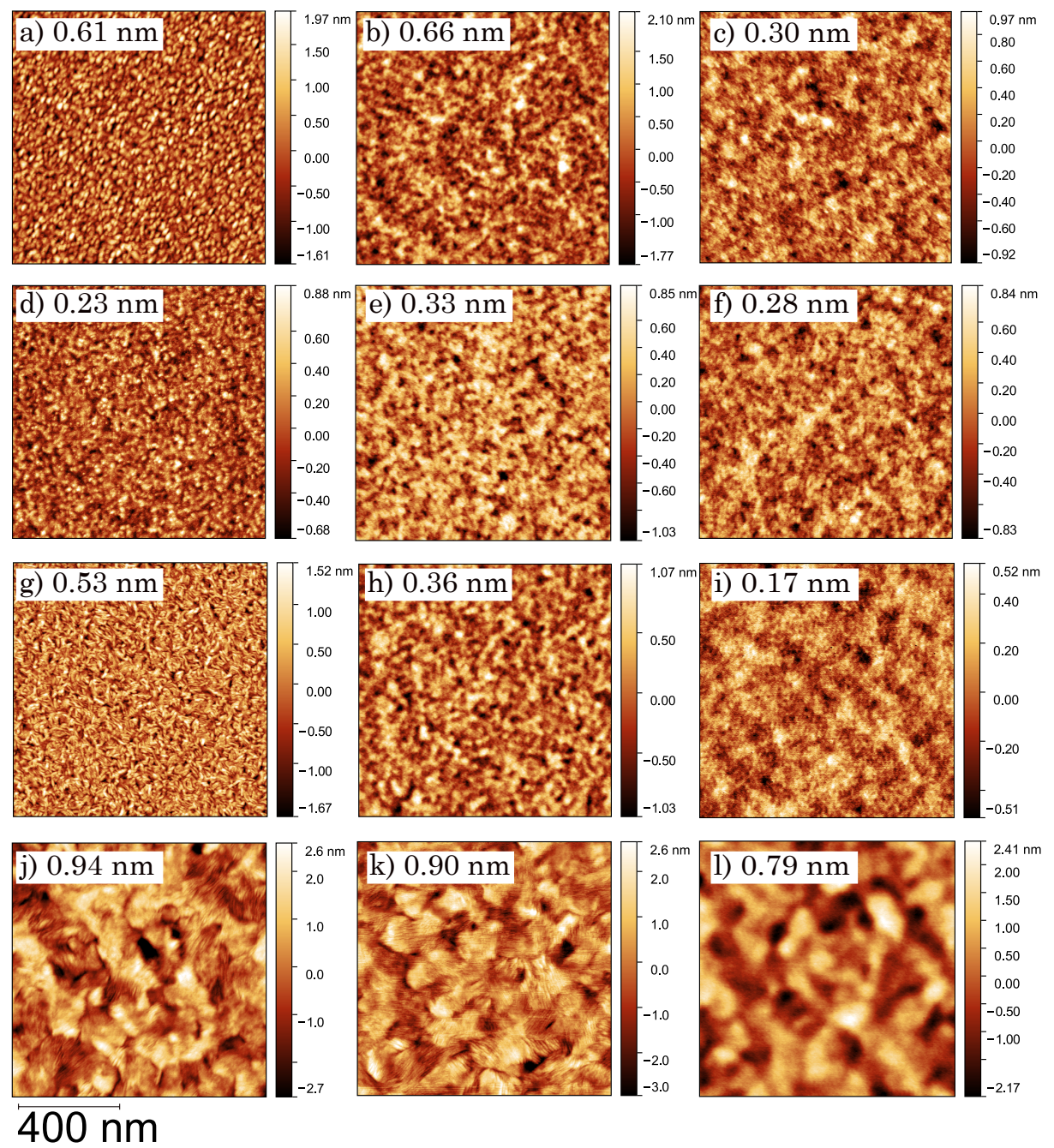

Figure A.2: $1 \mu m \times 1 \mu m$ atomic force micrographs of (a-c) palladium; (d-f) ruthenium; (g-i) molybdenum; and ( $\mathrm{j}-\mathrm{l})$ tungsten. The micrographs show, in increasing order: reference; $60 \mathrm{eV}$ $\mathrm{O}_{2}^{+} / \mathrm{O}^{+}$ion exposed and; $480 \mathrm{eV}$ (280 eV for palladium) $\mathrm{O}_{2}^{+} / \mathrm{O}^{+}$ion exposed surfaces. 



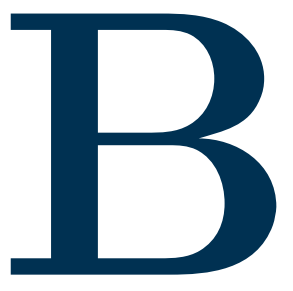

\section{BAyesian MCMC Results}

Here, we present results from the MCMC ensemble sampling of posterior probabilities for molybdenum, ruthenium, palladium and tungsten for argon and neon ion bombardment. Also included are maximum likelihoods of model parameters from $M C M C$ evaluations of literature and simulated sputter yields for a range of elements. 


\section{B.1 Posterior Probabilities}

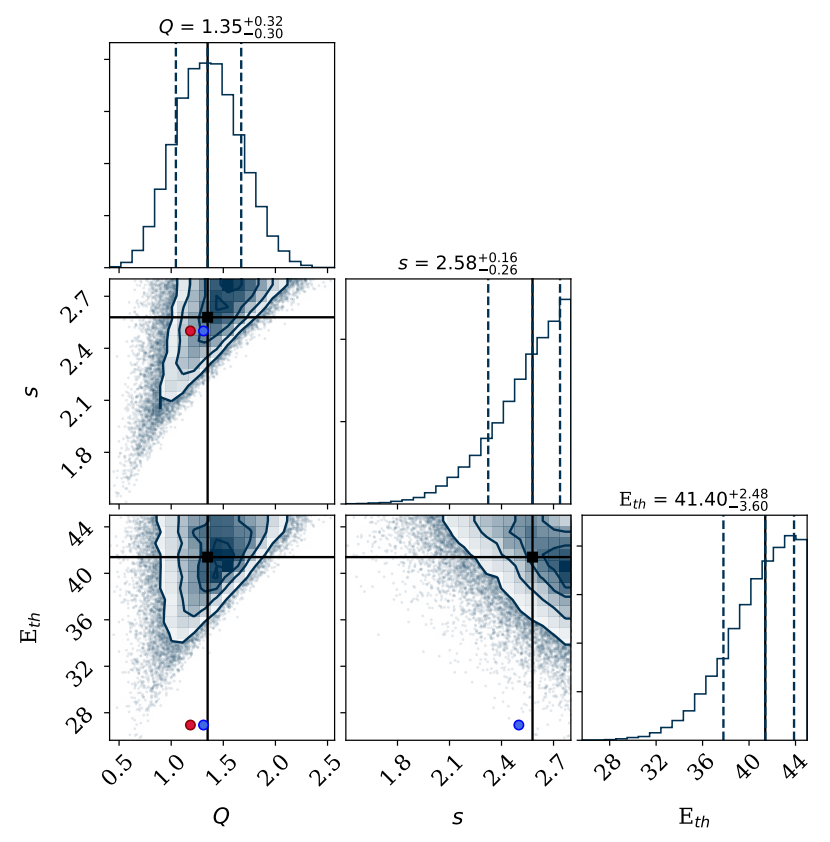

Figure B.1: Posterior probabilities for sputter yields of ruthenium (Ru) under $\mathrm{Ar}^{+}$ion bombardment. The red dot shows $Q_{e f f}$ and the blue dot shows the original tabulation by Yamamura.

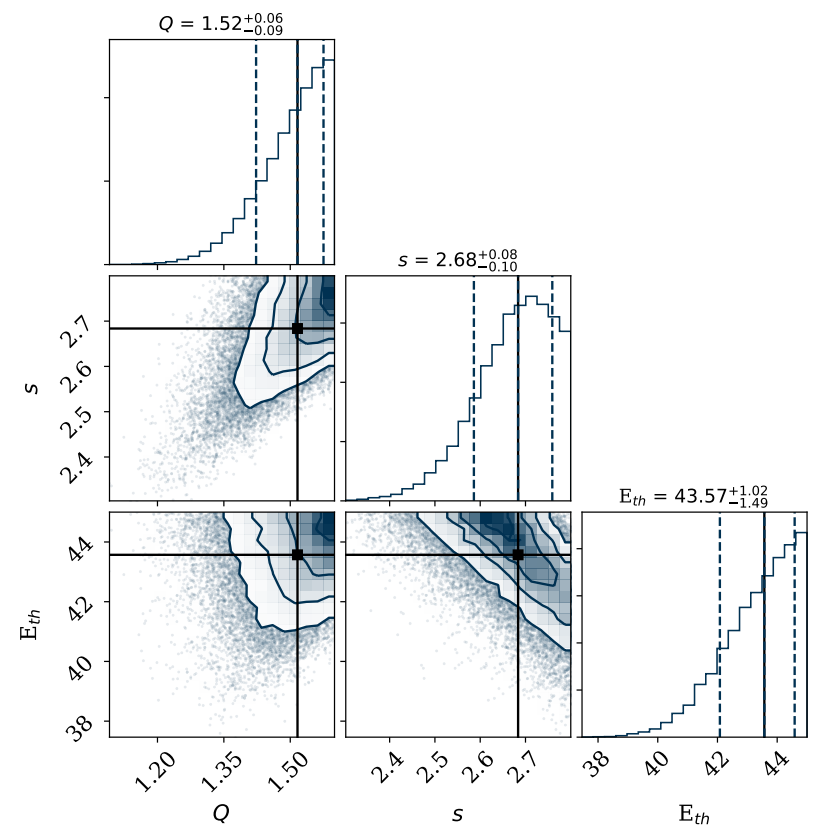

Figure B.2: Posterior probabilities for sputter yields of molybdenum (Mo) under $\mathrm{Ar}^{+}$ion bombardment. Tabulated $Q=0.85$ from Yamamura not pictured here. 


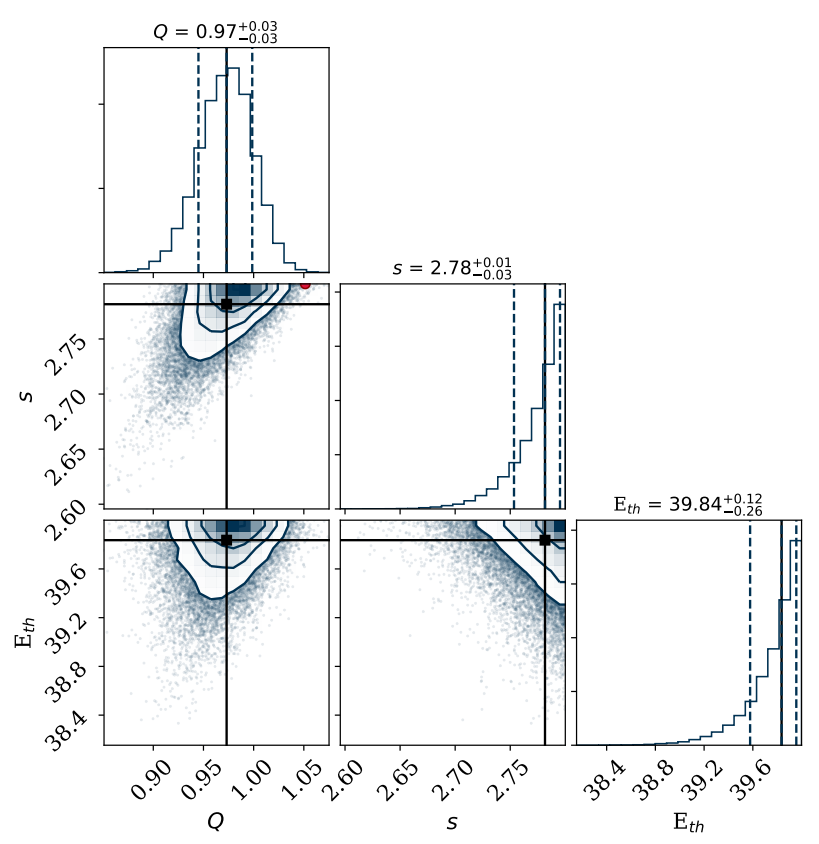

Figure B.3: Posterior probabilities for sputter yields of tungsten (W) under $\mathrm{Ar}^{+}$ion bombardment. The red dot shows $Q_{\text {eff }}$. Tabulated $Q=0.72$ from Yamamura not depicted.

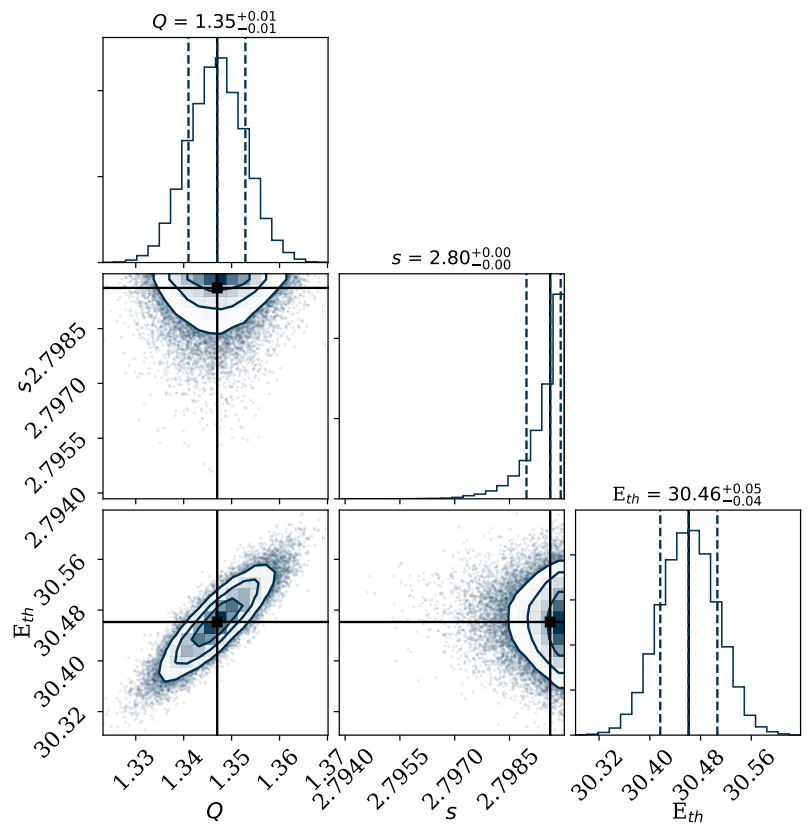

Figure B.4: Posterior probabilities for sputter yields of ruthenium (Ru) under $\mathrm{Ne}^{+}$ion bombardment. Tabulated $Q=1.31$ from Yamamura not pictured here. 


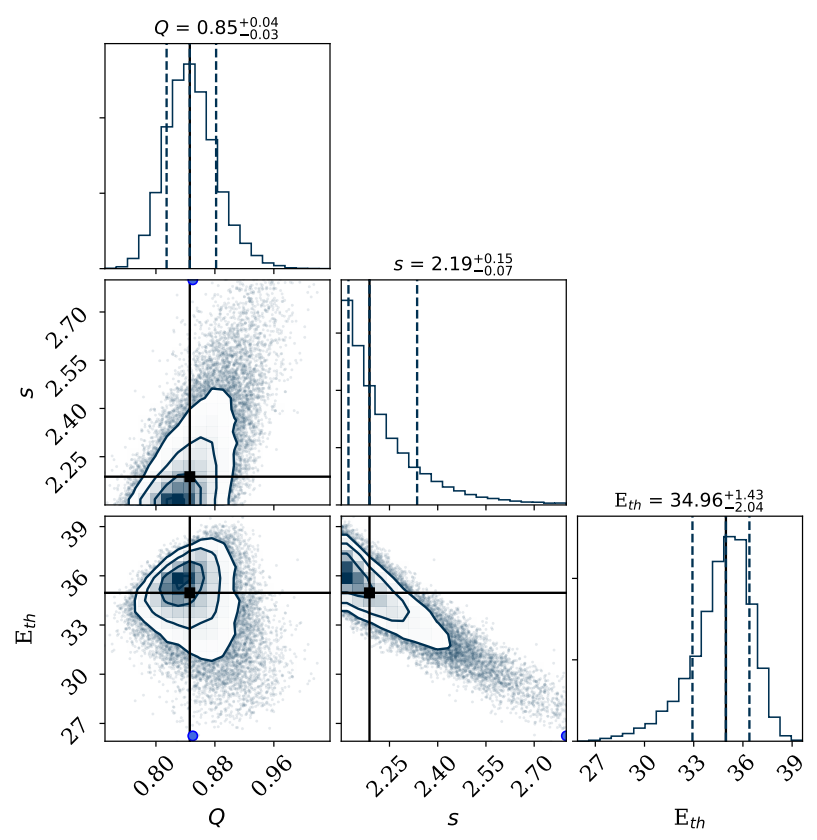

Figure B.5: Posterior probabilities for sputter yields of molybdenum (Mo) under $\mathrm{Ne}^{+}$ion bombardment. The blue dot shows the original tabulation by Yamamura.

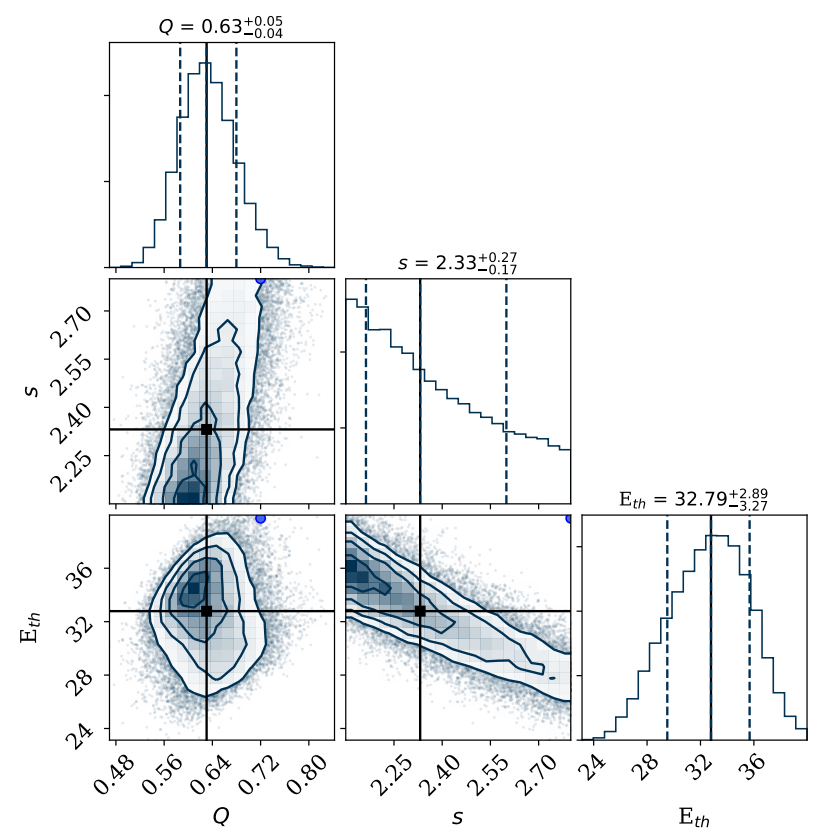

Figure B.6: Posterior probabilities for sputter yields of tungsten (W) under $\mathrm{Ne}^{+}$ion bombardment. The blue dot shows the original tabulation by Yamamura. 


\section{B.2 MCMC results for elements}

The maximum likelihoods for various elements studied in this work is presented in this chapter along with errors and previous literature values.

Table B.1: MCMC Results for Argon ion bombardment. Literature columns indicate $Q_{\text {eff }}$ values obtained from equation 6.5 in chapter 6. $s$ and $E_{t h}$ taken from Yamamura model. MCMC results performed for selected elements where experimental data was available and additional MCMC parameter space searches were performed on simulated yields.

\begin{tabular}{|c|c|c|c|c|c|c|c|c|c|c|c|}
\hline \multirow[b]{3}{*}{ Element } & \multirow[b]{3}{*}{ Mass } & \multirow[b]{3}{*}{$r^{3}$} & \multicolumn{3}{|c|}{ Literature } & \multicolumn{6}{|c|}{ MCMC (This Work) } \\
\hline & & & \multirow[b]{2}{*}{$Q_{e f f}$} & \multirow[b]{2}{*}{$s$} & \multirow[b]{2}{*}{$E_{t h}$} & \multicolumn{3}{|c|}{ Literature Data } & \multicolumn{3}{|c|}{ Simulated Data } \\
\hline & & & & & & $Q_{M C M C}$ & $s$ & $E_{t h}$ & $Q_{M C M C}$ & $s$ & $E_{t h}$ \\
\hline $\mathrm{Be}$ & 9 & 0.0081 & 2.00 & 2.5 & - & 2.60 & 2.51 & 59.9 & & & \\
\hline $\mathrm{Al}$ & 27 & 0.0166 & 1.41 & 2.5 & 23.6 & 1.20 & 2.63 & 23.5 & 0.95 & 2.67 & 29.3 \\
\hline $\mathrm{Si}$ & 28 & 0.0200 & 0.76 & 2.5 & 31.9 & 0.91 & 2.28 & 37.7 & 0.67 & 2.76 & 28.4 \\
\hline $\mathrm{Ti}$ & 48 & 0.0176 & 0.68 & 2.5 & 28.2 & 0.57 & 2.68 & 17.0 & & & \\
\hline $\mathrm{V}$ & 51 & 0.0142 & 0.78 & 2.5 & 29.5 & 0.69 & 2.37 & 25.1 & & & \\
\hline $\mathrm{Cr}$ & 52 & 0.0120 & 1.03 & 2.5 & 22.4 & 0.87 & 1.89 & 21.2 & & & \\
\hline $\mathrm{Fe}$ & 56 & 0.0118 & 0.76 & 2.5 & 22.3 & 1.03 & 2.57 & 31.3 & 1.19 & 2.63 & 29.0 \\
\hline $\mathrm{Ni}$ & 58 & 0.0108 & 1.17 & 2.5 & 22.5 & 1.11 & 2.20 & 25.1 & & & \\
\hline Co & 59 & 0.0110 & 1.03 & 2.5 & 22.2 & 1.06 & 2.77 & 20.7 & & & \\
\hline $\mathrm{Cu}$ & 63 & 0.0117 & 1.16 & 2.5 & 16.8 & 1.40 & 2.32 & 24.7 & & & \\
\hline $\mathrm{Ge}$ & 74 & 0.0230 & 0.51 & 2.5 & 17.4 & 0.64 & 2.65 & 13.8 & 0.70 & 2.77 & 18.8 \\
\hline $\mathrm{Zr}$ & 90 & 0.0230 & 0.78 & 2.8 & 25.8 & 0.71 & 2.55 & 26.2 & & & \\
\hline $\mathrm{Nb}$ & 93 & 0.0180 & 0.86 & 2.8 & 31.1 & 0.80 & 2.54 & 28.1 & & & \\
\hline Mo & 98 & 0.0160 & 0.99 & 2.8 & 27.7 & 0.72 & 1.76 & 32.4 & & & \\
\hline $\mathrm{Ru}$ & 102 & 0.0137 & 1.28 & 2.5 & 27.0 & 1.14 & 2.32 & 32.8 & & & \\
\hline $\mathrm{Rh}$ & 103 & 0.0138 & 0.15 & 2.5 & 22.9 & 1.23 & 2.52 & 26.5 & & & \\
\hline $\mathrm{Pd}$ & 106 & 0.0146 & 1.11 & 2.5 & 15.4 & 1.26 & 2.27 & 25.2 & 0.91 & 2.69 & 19.5 \\
\hline $\mathrm{Ag}$ & 107 & 0.0017 & 1.11 & 2.8 & 11.6 & 1.34 & 2.63 & 17.0 & & & \\
\hline Sn & 120 & 0.0274 & $0.61 *$ & 2.5 & 12.2 & - & - & - & 0.57 & 2.47 & 15.3 \\
\hline $\mathrm{La}$ & 139 & 0.0349 & 0.43 & 2.5 & 16.8 & - & - & - & 0.52 & 2.76 & 17.9 \\
\hline $\mathrm{Ce}$ & 140 & 0.0225 & $0.47^{*}$ & 2.5 & - & - & - & - & 0.52 & 2.74 & 17.2 \\
\hline $\mathrm{Hf}$ & 180 & 0.0225 & $0.67^{*}$ & 2.5 & 24.5 & 0.61 & 2.56 & 17.8 & 0.60 & 2.78 & 19.8 \\
\hline $\mathrm{Ta}$ & 181 & 0.0181 & 0.85 & 2.8 & 30.8 & 0.80 & 2.08 & 30.1 & 0.78 & 2.79 & 28.8 \\
\hline $\mathrm{W}$ & 184 & 0.0158 & 0.96 & 2.8 & 34.0 & 0.74 & 2.01 & 32.4 & & & \\
\hline Os & 192 & 0.0142 & 1.11 & 2.5 & 31.3 & 0.98 & 2.47 & 26.5 & 0.87 & 2.79 & 29.9 \\
\hline $\mathrm{Pt}$ & 195 & 0.0151 & 1.07 & 2.5 & 22.4 & 1.16 & 2.41 & 25.9 & & & \\
\hline $\mathrm{Au}$ & 197 & 0.0169 & 1.12 & 2.8 & 14.6 & 1.32 & 2.52 & 22.5 & & & \\
\hline $\mathrm{Th}$ & 232 & 0.0331 & - & 2.5 & 24.5 & 0.46 & 2.35 & 21.6 & & & \\
\hline $\mathrm{U}$ & 238 & 0.0208 & - & 2.5 & 22.1 & 0.64 & 2.42 & 24.7 & & & \\
\hline
\end{tabular}


Table B.2: MCMC Results for Neon ion bombardment. $s$ and $E_{t h}$ in the Literature column taken from Yamamura model. MCMC results performed for selected elements where experimental data was available and additional MCMC parameter space searches were performed on simulated yields.

\begin{tabular}{|c|c|c|c|c|c|c|c|c|c|c|c|}
\hline \multirow[b]{3}{*}{ Element } & \multirow[b]{3}{*}{ Mass } & \multirow[b]{3}{*}{$r^{3}$} & \multicolumn{3}{|c|}{ Literature } & \multicolumn{6}{|c|}{ MCMC (This Work) } \\
\hline & & & \multirow[b]{2}{*}{$Q_{e f f}$} & \multirow[b]{2}{*}{$s$} & \multirow[b]{2}{*}{$E_{t h}$} & \multicolumn{3}{|c|}{ Literature Data } & \multicolumn{3}{|c|}{ Simulated Data } \\
\hline & & & & & & $Q_{M C M C}$ & $s$ & $E_{t h}$ & $Q_{M C M C}$ & $s$ & $E_{t h}$ \\
\hline $\mathrm{Be}$ & 9 & 0.0081 & 1.51 & 2.5 & - & 2.65 & 2.39 & 82.0 & & & \\
\hline $\mathrm{Al}$ & 27 & 0.0166 & 0.83 & 2.5 & 18.2 & 1.27 & 2.07 & 66.5 & 0.88 & 2.53 & 19.1 \\
\hline $\mathrm{Si}$ & 28 & 0.0200 & 0.78 & 2.5 & 24.2 & 1.03 & 2.10 & 46.2 & 0.77 & 2.27 & 32.1 \\
\hline $\mathrm{Ti}$ & 48 & 0.0176 & 0.49 & 2.5 & 19.8 & 0.52 & 2.24 & 22.7 & & & \\
\hline $\mathrm{V}$ & 51 & 0.0142 & 0.67 & 2.5 & 21.3 & 0.69 & 2.46 & 25.0 & & & \\
\hline $\mathrm{Cr}$ & 52 & 0.0120 & 0.99 & 2.5 & 16.3 & 1.01 & 2.16 & 25.4 & & & \\
\hline $\mathrm{Fe}$ & 56 & 0.0118 & 0.62 & 2.5 & 16.8 & 0.83 & 2.37 & 22.6 & 1.15 & 2.6 & 19.2 \\
\hline $\mathrm{Ni}$ & 58 & 0.0108 & 0.96 & 2.5 & 17.3 & 0.80 & 1.94 & 12.5 & & & \\
\hline Co & 59 & 0.0110 & 1.11 & 2.5 & 17.0 & 1.03 & 2.49 & 25.9 & & & \\
\hline $\mathrm{Cu}$ & 63 & 0.0117 & 1.07 & 2.5 & 13.4 & 1.67 & 2.54 & 26.8 & & & \\
\hline $\mathrm{Ge}$ & 74 & 0.0230 & 0.47 & 2.5 & 14.6 & 0.54 & 2.52 & 12.7 & 0.68 & 2.57 & 14.6 \\
\hline $\mathrm{Zr}$ & 90 & 0.0230 & 0.62 & 2.8 & 23.8 & 0.55 & 2.42 & 24.8 & & & \\
\hline $\mathrm{Nb}$ & 93 & 0.0180 & 0.68 & 2.8 & 28.9 & 0.69 & 2.60 & 27.1 & & & \\
\hline $\mathrm{Rh}$ & 103 & 0.0138 & 0.88 & 2.5 & 22.2 & 0.87 & 2.31 & 23.5 & & & \\
\hline $\mathrm{Ag}$ & 107 & 0.0170 & 0.96 & 2.8 & 11.5 & 1.13 & 2.22 & 23.3 & & & \\
\hline In & 115 & 0.0262 & - & 2.5 & 9.9 & - & - & - & 0.56 & 2.62 & 9.7 \\
\hline Sn & 120 & 0.0274 & - & 2.5 & 12.4 & - & - & - & 0.55 & 2.39 & 13.4 \\
\hline $\mathrm{La}$ & 139 & 0.0374 & - & 2.5 & 18.2 & - & - & - & 0.49 & 2.68 & 16.5 \\
\hline $\mathrm{Ce}$ & 140 & 0.0349 & - & 2.5 & - & - & - & - & 0.80 & 2.72 & 19.1 \\
\hline $\mathrm{Hf}$ & 180 & 0.0225 & 0.51 & 2.5 & 29.0 & 0.50 & 2.55 & 26.1 & 0.59 & 2.30 & 29.4 \\
\hline W & 184 & 0.0158 & 0.76 & 2.8 & 40.5 & 0.50 & 2.20 & 24.7 & 0.72 & 2.78 & 29.8 \\
\hline Os & 192 & 0.0142 & 0.67 & 2.5 & 37.8 & 0.69 & 2.33 & 32.4 & 0.79 & 2.35 & 38.9 \\
\hline $\mathrm{Pt}$ & 195 & 0.0151 & 0.75 & 2.5 & 27.3 & 0.74 & 2.22 & 22.9 & & & \\
\hline $\mathrm{Au}$ & 197 & 0.0169 & 0.82 & 2.8 & 17.9 & 0.76 & 2.24 & 15.5 & 0.65 & 2.36 & 19.6 \\
\hline $\mathrm{U}$ & 238 & 0.0208 & - & 2.5 & 28.6 & 0.57 & 2.52 & 26.4 & & & \\
\hline
\end{tabular}






\section{VALORIZATION AND OUTLOOK}

Understanding the interactions of reactive ion species with thin metal films allows for control of film composition and thickness. This has potential applications in a wide range of natural and industrial processes where low energy ions interact with surfaces of devices or protective layers. Coolant gases such as $\mathrm{N}_{2}$ used in fusion reactors for plasma detachment ionize and react with divertor (tungsten) walls [1]. The ion energies here are $<1 \mathrm{keV}$ and can modify divertor composition leading to changes in thermal properties, mechanical properties as well as fuel retention capacity. Sensors in low-earth orbit satellites corrode under low energy $(5-100 \mathrm{eV})$ oxygen ion [2,3] irradiation created in the surrounding atmosphere. Control can be achieved by either tuning tolerances in materials exposed to harsh natural/industrial environments or by developing materials resistant to the processes involved. Thus, the results from this work on compound formation and sputtering of metals under low-energy reactive ion sputtering (Chapter 4 and Chapter 5) finds relevance for a wide range of applications as above.

Sputter yields are also of relevance where material loss is of critical importance. Sputter depth profile analysis employed in metrology such as X-Ray photoelectron spectroscopy, low-energy ion scattering, etc., in many cases requires knowledge of sputter yields of target materials under low energy inert gas ion bombardment [4]. With ion energies pushed to lower ranges to reduce beam induced artefacts, the knowledge of sputter yields becomes critical in depth quantification. Further, for applications of ion beam deposition, where high flux, low energy ions are employed, availability of precise sputter yields aids in control of thickness and composition of the deposited layer. The results of sputter yields and elements studied in this thesis (Chapter 6) are potentially beneficial for a wide range of applications demanding thin films such as sensors and transistor elements.

This work led to insights into transport processes of reactive ion species in metallic targets. The balance between material removed and implanted was found to be governed by primarily ballistics in the case of nitrogen ion bombardment. However, for oxygen, additional diffusion processes were found to be relevant in the transport. This leads to open questions such as: what is the driving contributor for diffusion-dominant and diffusion-'recessive' transport processes that differentiates oxygen transport from nitrogen transport in metals? Further, what is the nature of the thermal component in the radiation enhanced diffusion of oxygen in metal targets? Answers to such questions can impart understanding of key mechanisms of ion beam induced oxidation of metals and allow for control of properties of thin films and plasma facing components. 


\section{References}

[1] R. Perillo, R. Chandra, G. R. A. Akkermans, W. A. J. Vijvers, W. A. A. D. Graef, I. G. J. Classen, J. van Dijk, and M. R. de Baar, Studying the influence of nitrogen seeding in a detached-like hydrogen plasma by means of numerical simulations, Plasma Physics and Controlled Fusion 60, 105004 (2018).

[2] B. Singh, L. Amore, W. Saylor, and G. Racette, Laboratory simulation of low earth orbital atomic oxygen interactionwith spacecraft surfaces, in 23rd Aerospace Sciences Meeting (1985) https://arc.aiaa.org/doi/pdf/10.2514/6.1985-477 .

[3] B. A. Banks, S. K. Rutledge, P. E. Paulsen, and T. J. Steuber, Simulation of the low earth orbital atomic oxygen interaction with materials by means of an oxygen ion beam, NASA Technical Memorandum, 101971 (1989).

[4] M. P. Seah, C. A. Clifford, F. M. Green, and I. S. Gilmore, An accurate semi-empirical equation for sputtering yields $i$ : for argon ions, Surface and Interface Analysis 37, 444 (2005), https://onlinelibrary.wiley.com/doi/pdf/10.1002/sia.2032 . 


\section{SUMMARY}

This thesis is dedicated to further the understanding of processes involved in ionsurface interactions at energies near the sputter threshold at normal incidence. Ballistic interactions lead to removal of target material (sputtering), or implantation of incident ion species in sub-surface regions. For reactive ions, compound formation and diffusion can additionally occur changing surface and sub-surface composition and lead to ion transport further into the target. Understanding these processes provides control over the target thickness and composition which is useful in processes of thin film deposition and processing applications. Further, it is also beneficial for uncontrolled processes to predict possible failure in plasma facing components. In this work, ballistic and diffusional transport processes were studied using a combination of experiments and Monte Carlo simulations. Experiments using thin-film targets grown on quartz crystal micro-balances and silicon wafers allowed for estimation of sputter efficiencies and compositional changes, respectively. Monte Carlo simulations using the TRIDYN code then provided a direct comparison of sputter efficiencies and a comparison of ion transport via reconstructions of XPS intensity ratios with simulated profiles or additionally, thickness values.

The use of broad beam, gridded, ion sources is common for purposes of etching wafers or ion assisted deposition as they provide high ion fluxes as well as a homogeneous exposure of samples that are several centimeters large. However, their use for determining sputter yields is not without risk. We have found that energetic neutrals pose an impediment for quantitative assessments by comparing sputter yields measured using DC and RF ion sources. The grid design can be detrimental at energies near the sputter threshold and the sources need to be coupled to neutral and mass filters for reliable experimentation.

Following assessments of ion sources and choosing an appropriate design, sputter yields were measured for transition metals: molybdenum, ruthenium, palladium and tungsten. It was hypothesized from initial results of ruthenium sputtering by nitrogen ions that the formation of a nitride causes a surface binding energy change leading to observed yields being lower than predictions of semi-empirical models. Further experimentation and simulation with TRIDYN showed modification of ballistics and collision cascades due to incorporation of lighter nitrogen atoms in sub-surface regions of ruthenium and palladium is responsible for the changes in sputter yields. The ability to demarcate these differences is an important advantage of TRIDYN simulations over semi-empirical models which do not directly account for accumulation induced density variations. Compound formation in each metal was observed to varying extents. Nitrogen incorporation was found to be explained by two modes: one where nitrogen has a high short range lateral diffusion with adsorption on both metal and nitrogen sites until saturation (molybdenum and tungsten); and another where nitrogen is incorporated only on metal sites leading 
to slow incorporation while the target remains mostly metallic (ruthenium and palladium). Over an energy range of $50-500 \mathrm{eV}$, the overall nitrogen concentration was found to be virtually independent of energy which was attributed to a balance between sputtering and incorporation. The combination of experimental results with TRIDYN simulations allowed an estimation of reaction cross-sections of the targets over the studied energy range. Tungsten showed the highest reactivity, followed by molybdenum, and lowest being both ruthenium and palladium whose cross-sections were similar.

Exposure of metal films mentioned above to oxygen ions showed significantly different behavior. Ruthenium was found to exhibit an anomalously high sputter efficiency upon oxygen ion bombardment which can potentially be due to volatile $\mathrm{RuO}_{4}$ formation from minuscule quantities of ozone produced in the oxygen plasma. Molybdenum and tungsten yields were also found to be higher than predictions of TRIDYN due to possible volatile compounds formed during ion exposure. Palladium showed yields well predicted by TRIDYN. All metals oxidized upon irradiation by oxygen ions with varying depths of oxygen transport. Oxygen transport and retention sub-surface was measured using angle resolved XPS and the depth of ion penetration was found to be generally under-estimated by purely ballistic transport. A diffusion algorithm was developed to couple to the ballistic transport profiles generated by TRIDYN. A radiation enhanced diffusion of oxygen by formation of highly mobile free oxygen atoms due to oxygen ion irradiation is expected to be a potential mechanism for ion transport within the target well past the ballistic range. This behavior is significantly different from the transport of nitrogen ions which requires further investigation.

Finally, inert ion induced sputtering of elements in general and transition metals in particular was evaluated. Experiments were performed at near sputter threshold energies, not reported in the literature so far. Inert ion species mainly contribute to sputtering with implantation being minimal (a few percent at most). They are thus prime candidates for assessment of ballistic transport and validation of semi-empirical formulae where dynamic effects are not taken into account. For the materials studied above, sputter yields were also measured using argon and neon ions and compared to the Yamamura model. The parameters and correlations between them were assessed using a Bayesian Markov Chain Monte Carlo approach. 'Best-fits' of model parameters were then compared to fits to literature data for a slew of elements in the periodic table. Fits performed to simulated yields to several elements additionally provided a means to compare an average behavior of the model parameters. It was found that the linear scaling of sputter yields (parameter Q) - which was formulated to be dependent on target density - exhibits oscillations when rescaled to the energy transfer parameter $(\gamma)$. These oscillations form bands that seemed to be governed by the electronic filling of orbitals. Such a rescaling allowed for ascribing a functional form to the variation in $\mathrm{Q}$ as a function of $\gamma$ that can be applied for argon and neon which was not done in previous formulations in literature. 


\section{SAMENVATting}

Het onderzoek beschreven in dit proefschrift richt zich op een beter begrip van processen gerelateerd aan ion-oppervlakinteracties bij normale inval met energieën in de buurt van de sputterdrempel. Ballistische wisselwerkingen leiden tot verwijdering van materiaal van de trefplaat (sputteren), of implantatie van invallende ionen in lagen direct onder het oppervlak. In het geval van reactieve ionen kan de samenstelling van het oppervlak en onderliggende atoomlagen bovendien veranderen door chemische interacties van de trefplaat met de ionen en diffusie van geïmplanteerde ionen door het materiaal. Een beter begrip van deze processen is nuttig voor toepassingen als depositie van dunne films en oppervlaktemodificatie, om zo de gewenste laagdikte en compositie van de gemodificeerde laag te bereiken. Bij materialen die aan plasma blootgesteld worden, helpt begrip van deze processen bij het voorspellen van een mogelijk falen van deze componenten. In dit onderzoek zijn ballistische processen en diffusieprocessen bestudeerd met een combinatie van experimenten en Monte-Carlosimulaties. Experimenten met dunne films gedeponeerd op respectievelijk kwarts microbalansen en siliciumsubstraten, zijn gebruikt om de sputteropbrengst en de veranderingen in samenstelling van de dunne film te meten. De experimentele sputteropbrengst kon direct vergeleken worden met Monte-Carlosimulaties met de TRIDYN code. Daarnaast zijn simulaties van het ionentransport vergeleken door gesimuleerde implantatieprofielen te vergelijken met metingen met Röntgen-foto-elektronspectroscopie (XPS) en daaruit bepaalde diktes.

Ionenkanonnen voorzien van roosters voor extractie van brede bundels worden veel gebruikt voor bijv. het etsen van siliciumplakken of ionengeassisteerde depositie, aangezien deze bronnen hoge ionenfluxen kunnen genereren en daarbij een gebied van vele centimeters groot homogeen kunnen bestralen. Het gebruik van dit type ionenbundels voor het bepalen van de sputteropbrengst is niet zonder risico. In dit onderzoek hebben we vastgesteld dat energetische neutralen het kwantitatief bepalen van de sputteropbrengst onmogelijk kunnen maken, door metingen van de sputteropbrengst met gelijkstroom (DC) en radiofrequente (RF) ionenbronnen te vergelijken. Verschillen in het ontwerp van de roosters van deze bronnen kunnen dramatische afwijkingen geven bij energieën in de buurt van de sputterdrempel, waardoor het bij bepaalde bronnen nodig kan zijn om massafilters en filters voor energetische neutralen te gebruiken.

$\mathrm{Na}$ het karakteriseren van deze ionenbronnen en de keuze voor een geschikt ontwerp, is de sputteropbrengst gemeten voor de overgangsmetalen molybdeen, ruthenium, palladium en wolfraam. De eerste resultaten van het sputteren van ruthenium door stikstofionen leidden tot de hypothese dat de vorming van een nitridelaag een vergroting van de oppervlaktebindingsenergie veroorzaakt, wat een lagere sputteropbrengst in vergelijking met de voorspelling door semi-empirische modellen zou 
verklaren. Vervolgexperimenten en simulaties met TRIDYN toonden aan dat veranderingen in de ballistische processen en botsingscascades door de incorporatie van lichtere stikstofatomen de sputteropbrengst van ruthenium en palladium kunnen verklaren. De mogelijkheid om deze processen te simuleren is een belangrijk voordeel van TRIDYN ten opzichte van semi-empirische modellen, die niet op directe wijze de invloed van incorporatie van ionen en/of dichtheidsveranderingen mee kunnen nemen. De mate waarin ionenbombardement leidt tot vorming van chemische verbindingen was afhankelijk van het soort metaal. De opname van stikstof kan verklaard worden door middel van twee modi: één waarbij stikstof een hoge laterale diffusiesnelheid over korte afstanden heeft, tot verzadiging optreedt (bij molybdeen en wolfraam) en een andere waarbij stikstof alleen op niet-gereageerde metaalatomen opgenomen kan worden, wat leidt tot langzame opname, waarbij de trefplaat voornamelijk metallisch blijft (bij ruthenium en palladium). In een energiebereik van 50-500 eV lijkt de totale stikstofconcentratie vrijwel onafhankelijk van de energie, wat toegeschreven kan worden aan een evenwicht tussen sputteren en incorporatie. Door experimentele resultaten te vergelijken met simulaties met TRIDYN, konden de werkzame doorsnedes voor reactie van stikstofionen in de bestudeerde metalen bepaald worden. Wolfraam vertoonde de hoogste reactiviteit, gevolgd door molybdeen, ruthenium en palladium, waarbij de reactiviteit van de laatste twee metalen vergelijkbaar was.

Bovengenoemde metaalfilms gedragen zich significant anders bij blootstelling aan zuurstofionen. Ruthenium vertoont een buitengewoon hoge sputteropbrengst bij bestraling door zuurstofionen, wat mogelijk verklaard kan worden door vorming van vluchtig $\mathrm{RuO}_{4}$ onder invloed van zeer kleine hoeveelheden ozon in het zuurstofplasma van de ionenbron. Molybdeen en wolfraam hebben eveneens een hogere sputteropbrengst dan de voorspellingen van TRIDYN, door mogelijke vorming van vluchtige verbindingen tijdens het ionenbombardement. Bij palladium is de sputteropbrengst in goede overeenstemming met de simulatie door TRIDYN. Alle metalen vormen oxide als gevolg van de bestraling, waarbij de gevormde oxidedikte materiaalafhankelijk is. Transport en retentie van zuurstof is gemeten met hoekopgeloste XPS, waaruit bleek dat de penetratiediepte over het algemeen onderschat wordt wanneer uitsluitend rekening gehouden wordt met ballistisch transport. Daarom is een diffusiealgoritme ontwikkeld dat gekoppeld kon worden aan de ballistische transportprofielen zoals die door TRIDYN gegenereerd worden. Op basis van dit algoritme, is de verwachting dat er ionengeïnduceerde diffusie optreedt, waarbij zeer mobiele zuurstofatomen kunnen leiden tot ionentransport in het materiaal, tot voorbij het bereik dat op basis van ballistische berekeningen voorspeld wordt. Dit gedrag is significant verschillend in vergelijking met het transport van stikstof en behoeft daarom verder onderzoek.

Tot slot is het sputteren van verscheidene elementen, met name overgangsmetalen, door edelgasionen bestudeerd. Hierbij is experimenteel de sputteropbrengst gemeten in de buurt van de sputterdrempel, waar weinig of geen experimentele resultaten bekend waren in de literatuur. Een bombardement door edelgasionen resulteert voornamelijk in sputteren, met minimale implantatie (maximaal een paar procent). Edelgasionen zijn daarom bij uitstek geschikt om ballistisch transport te onderzoeken en semi-empirische formules voor de sputteropbrengst te verifiëren, 
aangezien de achterliggende theorie geen rekening houdt met dynamische verandering van het bestraalde materiaal. De sputteropbrengst van de eerder genoemde metalen door argon en neon is daartoe gemeten en vergeleken met het Yamamuramodel. De parameters en onderlinge correlaties zijn bepaald met een zogenaamde "Bayesian Markov Chain" Monte-Carlomethode. De "beste overeenkomsten" van de modelparameters zijn vervolgens vergeleken met fits van de experimentele sputteropbrengst van een veelvoud aan elementen waarvoor literatuurdata bekend is. Waar geen literatuurdata beschikbaar was, is de sputteropbrengst gesimuleerd met TRIDYN en vervolgens gefit met het Yamamura-model. Uit deze analyse kon worden afgeleid dat de lineaire schalingsparameter van de sputteropbrengst $Q$ - die eerder geformuleerd was als functie van de materiaaldichtheid - een oscillerende functie vertoont wanneer $\mathrm{Q}$ wordt uitgezet tegen de energieoverdrachtsparameter $\gamma$. Deze oscillaties lijken gedomineerd te worden door het vullen van elektronische orbitalen met toenemende atomaire massa. Deze benadering om Q te beschrijven als functie van $\gamma$ geeft de mogelijkheid om een min of meer universele functie van $\mathrm{Q}$ te beschrijven die zowel toegepast kan worden voor argon als neon, wat niet eerder beschreven is in de literatuur. 



\section{ACKNOWLEDGeMents}

Looking back at the end of an expedition I feel a need express gratitude towards all the people who showed me the path (or warned me against treacherous terrain), as well as others I met along the way who made the journey enjoyable. Without them, this thesis would not have been possible.

Firstly, I would like to thank Prof. Fred Bijkerk for the opportunity to pursue a $\mathrm{PhD}$ in the $\mathrm{XUV}$ optics group and for motivating me at crucial moments in the research. Your support and encouragement were valuable in the progress of the project.

I owe enormous gratitude to my daily supervisor Dr. Marko Sturm for his endless patience, support and freedom for creative ideas. You helped me wade through the waters of experimental setups with your expertise and a knack for simple and practical solutions. I hope to have learned much from you. Your support in the writing process was monumental and it always seemed as if my walls of text were the only ones on your reading list. A special thanks to Dr. Robbert van de Kruijs for his constructive criticism towards my problems and my writing. Your keen eye has made my manuscripts more robust (and less porous) and I am very grateful.

I would also like to thank other senior members of the XUV team, both past and present: Dr. Eric Louis, Dr. Andrey Yakshin, Dr. Igor Makhotkin, Dr. Muharrem Bayraktar and Dr. Chris Lee for their valuable discussions during and outside of internal meetings. I am thankful to Norbert Koster and Dr. Diederik Maas for their discussions in the initial stages of the project. Although the path kept changing, you supported the new routes we ended up pursuing. A big thanks to Dr. Hartmut Enkisch for your critical analysis of the results and constructive debates.

None of this would be possible without the diligence of Jacqueline Emmerich and Carin Krijnen. Thank you for your help with finding me a place to stay when I first arrived in the Netherlands and everything else later on. Without your skills, we'd quickly be thrust into chaos!

This work also benefited from the wise insights and guidance from external members. Many thanks to Prof. Dmitry Lopaev for your explanations on industrial plasma generators and ion source-ry. Prof. Wolfhard Möller for your guidance with troubleshooting the TRIDYN Monte Carlo code and interpreting results. I am grateful to Dr. Erwin Zoethout for insights, advice and fruitful discussions on surface reactions and characterizing thin film surfaces.

I owe a big thanks to Theo van Oijen for your help with the technical and practical aspects of thin-film coatings. Also to Koen Weghorst in the design of the ion exposure chamber and the relentless modifications that followed. Bart Spanjer for his technical assistance and Mike Dikkers for friendly discussions in the lab. Things were not always smooth sailing, but all of you made sure that we maneuvered safely through troubled waters. 
Over the course of my time at the XUV optics group, I was fortunate to meet colleagues mentioned above as well as fellow PhD students and Post-Docs. Among them, I am especially thankful to Dr. Konstantin Nikolaev for your warm friendship and help in difficult times. To Dr. Andrey Zameshin for your words of wisdom, friendship and help in the last leg of the PhD. Dr. Igor Milov for your support outside of the work sphere and regular reminders to rest and relax. To Cristiane Stilhano Vilas Boas for your support in scientific endeavors and your trusting friendship, thanks for keeping me sane under pressure. Anirudhan Chandrasekaran for making the office a pleasant and energetic place to work; with you, there was never a dull day. And to Dr. Dmitry Kuznetsov for our corridor conversations and parties full of cheerful songs. I owe thanks to Dennis Ijpes for your company and the many chess filled Friday evenings, may there be more to come. The company of Oleksandr Antonov, Zahra Heidarnia Fathabad, Olena Soroka, Airat Shafikov, Roman Medvedev, Dr. Benjamin Wylie van Eerd, Dr. Johan Reinink, and Dr. Wesley van den Beld, made working in the group a truly memorable experience.

On a personal note, I would like to thank friends outside of work and from India for their encouragement throughout my long journey: To Yelizaveta Gasyuk for helping me unwind with tea, conversations and evening strolls. A big thanks to Manila Chhotray and Sanjay Rawat for fostering me; I always had a place to call home away from home. To Mehul Bhatkoti and Siddharth Mukherjee for their heartfelt encouragement in difficult moments. And Amit Pandey for his relentless friendship, the world is a dimmer place without you.

I shall be eternally indebted to my parents Swati and Vivek Phadke. You taught me the values I know and gave me everything you could especially love and encouragement. I appreciate the support of Aniruddha and Mathangi Phadke; you made sure I did not have to worry about things at home and focus on my work. I am fortunate to have the mental and emotional support of Jainishka Shah; you bring a much-needed positive outlook to my life and this would not have been possible without you.

Finally, I would like to appreciate the efforts of my mentors in science and life. Most notably Dr. K. N. Krishnakumar for your dedication to teaching and imparting in me the skills of critical thinking; you incited my interest in science and I am forever grateful. Also to Dr. Santa Chawla for taking me under your wing, for honing my scientific acumen and for numerous life-lessons.

And thanks to you dear reader, for taking the time to get this far. While this thesis ends here, the work continues ... 


\section{Curriculum Vitae}

Parikshit Phadke was born on $28^{\text {th }}$ of August 1991 in Bhopal, India. Following his schooling in Hyderabad (India), he moved to Noida (India) in 2009 to pursue a degree in Nanotechnology from Amity University. During the course of his studies, he was involved in internships notably at the National Institute of Technology, Rourkela (NIT-R, India) where he worked on the synthesis of bi-metallic nano-particles for catalytic reactions. For his Master's thesis, he worked at the National Physical Laboratory, New Delhi. During this period he studied the plasmonic enhancement of photo-luminescence in phosphors by plasmonic nano-antennas and co-authored several publications. After successfully completing a Master's in Nanotechnology in 2014, he moved to the Netherlands in 2015 to pursue a $\mathrm{PhD}$ at the Industrial Focus Group XUV Optics in the MESA+ Institute of Nanotechnology, University of Twente. Under the supervision of Prof. Dr. Fred Bijkerk and Dr. Marko Sturm, he was involved in investigation of near-threshold sputtering and surface modifications by inert and reactive ion bombardment. Here, he gained crucial experience in experimental and simulation techniques; and contributed to journal publications and conference presentations with some selected works compiled in this thesis.

He currently works at the XUV Focus Group at the University of Twente, applying his expertise to investigate low energy phenomena in reactive ion bombardment on industrially relevant surfaces. 



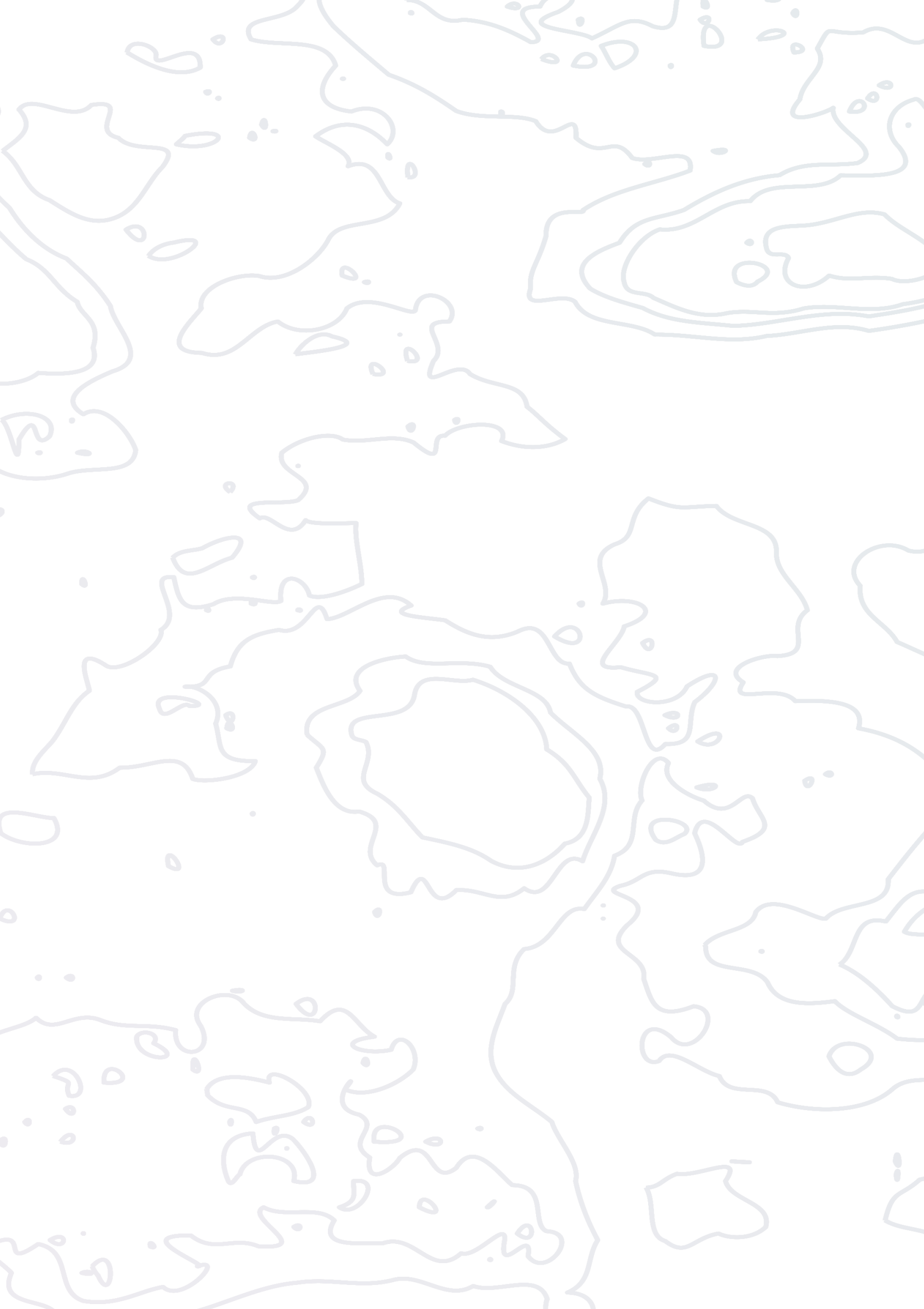


$\therefore 8$ O०

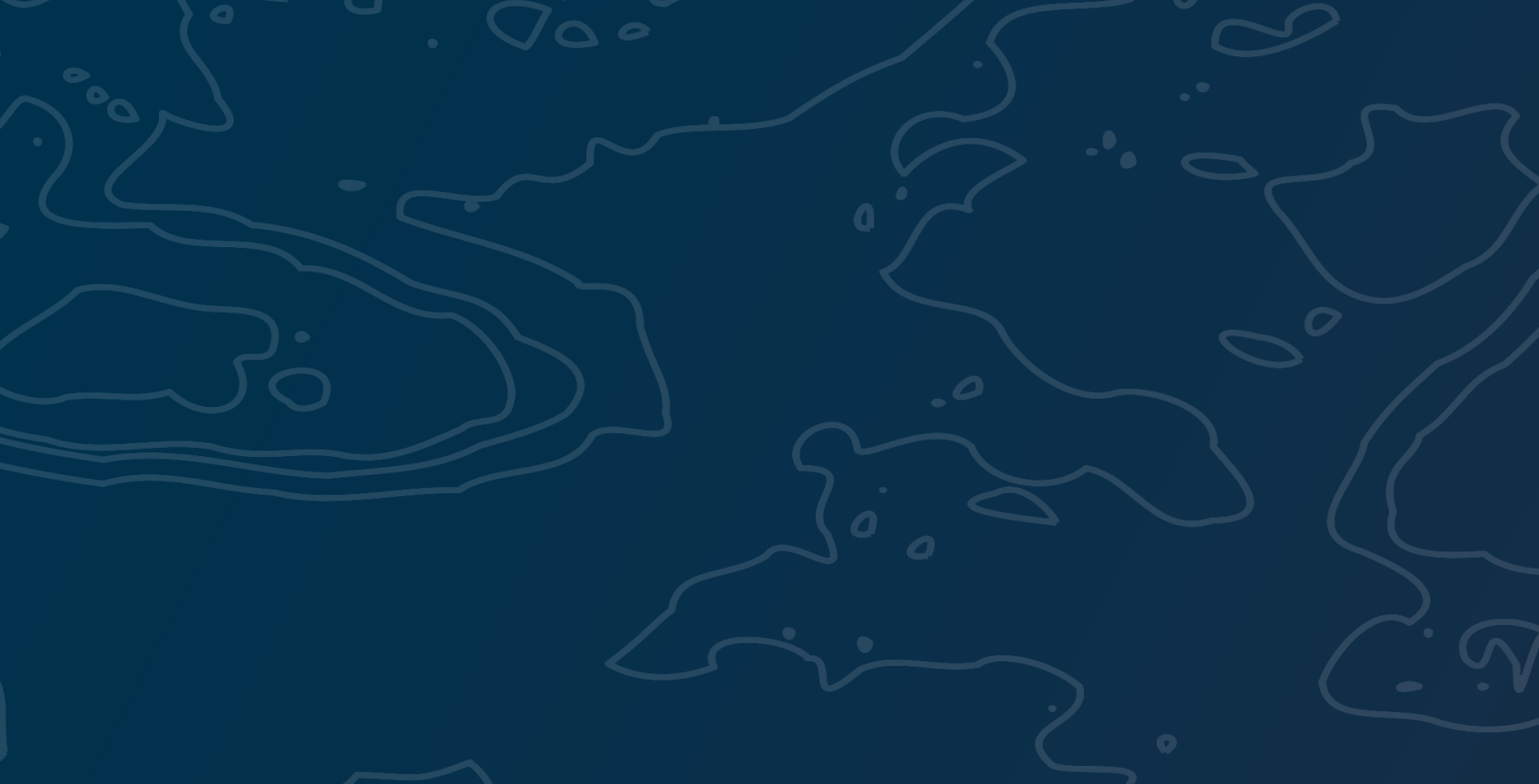

